\title{
Propositions
}

1. Ecosystem history needs to be explicitly represented in statistical data-driven models. (this thesis)

2. Deep neural networks are able to learn climate and vegetation's ecological memory effects for improving $\mathrm{CO}_{2}$ flux estimates.

(this thesis)

3. Machine learning algorithms are useful tools to tackle important scientific questions, but users often misunderstand or misuse such methods.

4. Disclosing conflict of interests in research is harmful for scientific publications.

5. Homo sapiens sapiens are social and cooperative animals; young leaders must initiate movements on contemporary issues regarding climate change, so the others will follow.

6. Displacing spatially and mentally food consumption from food production does not benefit the Earth nor the consumers.

Propositions belonging to the thesis, entitled:

\section{Controls of forest age and ecological memory effects on biosphere-atmosphere $\mathrm{CO}_{2}$ exchange}

Simon Besnard

Wageningen, June $7^{\text {th }} 2019$ 


\section{Controls of forest age and ecological memory effects on biosphere-atmosphere $\mathrm{CO}_{2}$ exchange}




\section{Thesis committee}

\section{Promotors:}

Prof. Dr M. Herold

Professor of Geo-information Science and Remote Sensing

Wageningen University \& Research

Prof. Dr M. Reichstein

Director of Department Biogeochemical Integration

Max Planck Institute for Biogeochemistry

\section{Co-promotors:}

\section{Dr J.G.P.W Clevers}

Associate professor, Laboratory of Geo-information Science and Remote Sensing Wageningen University \& Research

Dr N. Carvalhais

Group leader of the Model-Data Integration Group

Max Planck Institute for Biogeochemistry

\section{Other members:}

Prof. Dr W. Peters, Wageningen University \& Research

Dr S. Vicca, University of Antwerp, Belgium

Prof. Dr G. Camps-Valls, Universitat de València, Spain

Dr C.S.R Neigh, NASA Goddard Space Flight Center, United States of America

This research was conducted under the auspices of the C.T. de Wit Graduate School of Production Ecology \& Resource Conservation (PE\&RC) 


\section{Controls of forest age and ecological memory effects on biosphere-atmosphere $\mathrm{CO}_{2}$ exchange}

Simon Besnard

Thesis

submitted in fulfilment of the requirements for the degree of doctor at Wageningen University by the authority of the Rector Magnificus

Prof. Dr A.P.J. Mol, in the presence of the Thesis Committee appointed by the Academic Board to be defended in public on Friday 20 September at 11 a.m. in the Aula. 
Simon Besnard

Controls of forest age and ecological memory effects on biosphere-atmosphere $\mathrm{CO}_{2}$ exchange 198 pages.

PhD thesis, Wageningen University, Wageningen, the Netherlands (2019)

With references, with summary in English

ISBN 978-94-6395-065-7

DOI https://doi.org/10.18174/497385 


\section{Contents}

Page

Contents

$\begin{array}{lll}\text { Chapter } 1 \text { General introduction } & 1\end{array}$

Chapter 2 Quantifying the effect of forest age in annual net forest carbon balance 13

Chapter 3 The global forest carbon balance inferred from flux towers and forest age 41

Chapter 4 Memory effects of climate and vegetation affecting net ecosystem $\mathrm{CO}_{2}$ fluxes in global forests

Chapter 5 Dynamic and lag effects of climate and vegetation on biosphereatmosphere $\mathrm{CO}_{2}$ exchange: a global analysis

Chapter 6 General discussion

References

Summary

Acknowledgements

About the author

PE\&RC Training and Education Statement 

Chapter 1

\section{General introduction}




\subsection{Context: terrestrial ecosystem and the global carbon cycle}

The key components of all known life on Earth are based on carbon (C). The latter is exchanged and cycled between a series of reservoirs in the Earth System, such as the atmosphere, ocean layers, land and lithosphere, soil, freshwaters as well as rocks and sediments. This exchange, the so-called Earth's $\mathrm{C}$ cycle, is a fundamental element of the climate system and consists of (i) rapid $\mathrm{C}$ exchange among living organisms as well as (ii) long-term cycling of $\mathrm{C}$ through geologic processes. Over the long term, the exchange between the atmosphere and the biosphere (land and oceans) seems to maintain an equilibrium, keeping the Earth's temperature relatively stable and predictable (Kasting, 1989). However, changes in the C cycle caused by human activities (e.g. land use changes, fossil fuel emissions) have been observed since the industrial era, increasing the concentrations of carbon dioxide $\left(\mathrm{CO}_{2}\right)$ in the atmosphere $(+240 \pm 10 \mathrm{PgC}$, Ciais et al. (2014b), Fig. 1.1) and resulting in warmer temperatures on Earth. In addition, there is positive feedback between climate and the $\mathrm{C}$ cycle, suggesting that elevated atmospheric $\mathrm{CO}_{2}$ affects the climate system, but warming, in turn, is expected to further enhance the concentration of atmospheric $\mathrm{CO}_{2}$.

As such, there is currently a lot of interests within the scientific community to understand the $\mathrm{C}$ cycle because of the observed increase in levels of atmospheric $\mathrm{CO}_{2}$. Over the last years, our knowledge of the mechanisms controlling the $\mathrm{C}$ cycle has increased, in particular with regard to the role of terrestrial ecosystems on the $\mathrm{C}$ balance. Terrestrial ecosystem $\mathrm{CO}_{2}$ fluxes are mainly controlled by two $\mathrm{C}$ fluxes: photosynthesis and respiration. Through photosynthesis (gross primary productivity (GPP)) plants remove $\mathrm{CO}_{2}$ from the atmosphere. This assimilated $\mathrm{C}$ is then cycled through plant tissues, litter, and soil $\mathrm{C}$ to be further respired back into the atmosphere through autotrophic respiration ( $\mathrm{Ra}$ ) (plants), heterotrophic respiration (Rh) (soil micro-organisms and animal), and disturbance processes. GPP is the largest $\mathrm{CO}_{2}$ flux in ecosystems with $+123 \pm 8 \mathrm{PgC}_{\text {year }}{ }^{-1}$ globally (Beer et al., 2010). The net primary productivity (NPP) $\left(+56 \pm 14.3 \mathrm{PgC}\right.$ year $^{-1}$, Ito (2011) represents the net amount of C assimilated by plants after photosynthesis and autotrophic respirationin an ecosystem (NPP= GPP - Ra). The C accumulated in biomass and soils is around 450-650 $\mathrm{PgC}$ and 1,500-2,400 $\mathrm{PgC}$, respectively, with an additional $1700 \mathrm{PgC}$ stored in permafrost (Fig. 1.1, Ciais et al. (2014b)). C in ecosystems is not only released back into the atmosphere through ecosystem respiration (ER) (ER = Ra $+\mathrm{Rh}$ ) but also through fires (an average of $-2.16 \mathrm{PgC} \mathrm{year}^{-1}$ for the period 1997-2016, Werf et al. (2017)) or carried along from the river to the oceans $\left(-1.7 \mathrm{PgC} \mathrm{year}^{-1}\right.$, Fig. 1.1, Ciais et al. (2014b)). When considering the non-respiratory losses (i.e. fire $\mathrm{C}$ emissions, emissions of volatile organic carbon (VOC), and lateral transport), the global terrestrial net biome production (NBP) is around $+2.6 \pm 1.2 \mathrm{PgC} \mathrm{year}^{-1}$ (Fig. 1.1, Ciais et al. (2014b)). This suggests that the terrestrial land surface is currently a net sink of atmospheric $\mathrm{CO}_{2}$. However, this might change under future climate conditions and these changes would carry global implications (e.g. rising of sea level, 
increasing frequency and severity of extreme weather events, growing population movements), albeit of uncertain magnitude (Friedlingstein et al., 2013).

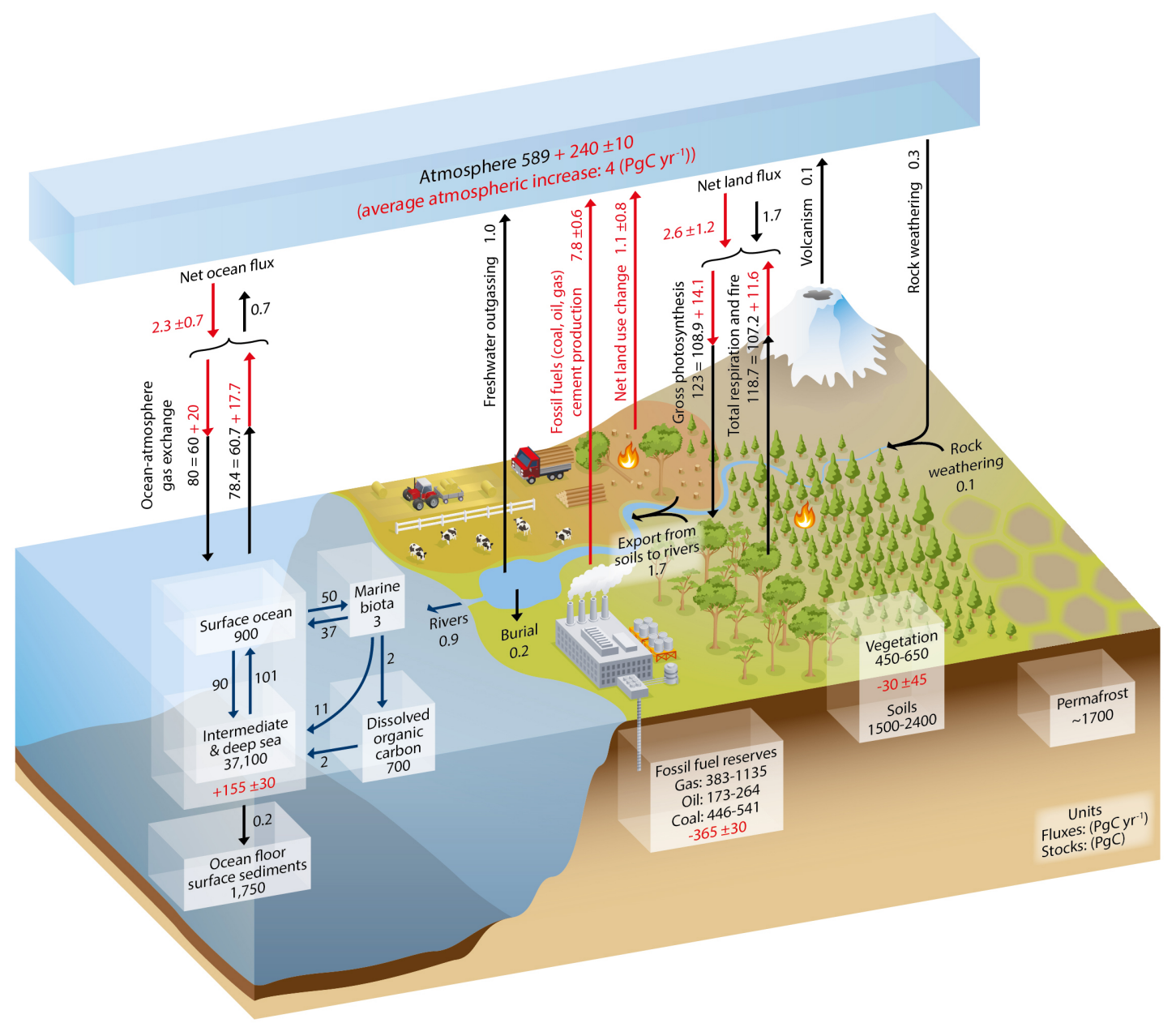

Figure 1.1: Schematic representation of the global C cycle (Ciais et al., 2014b). Numbers represent reservoir mass, also called ' $\mathrm{C}$ stocks' in $\mathrm{PgC}$ and annual $\mathrm{C}$ exchange fluxes (in $\mathrm{PgC} \mathrm{year}^{-1}$ ). Black numbers and arrows indicate reservoir mass exchange fluxes estimated for the time period prior to the industrial era, about 1750. More details about this figure can be found in Ciais et al. (2014b)

The net ecosystem production (NEP) is used as the key measure of metabolism and atmospheric $\mathrm{CO}_{2}$ exchange in terrestrial ecosystems. NEP represents the amount of atmospheric $\mathrm{CO}_{2}$ fixed that is available for biomass and detritus accumulation, export, or non-biological oxidation in an ecosystem (Lovett et al., 2006) and is generally defined as the difference between GPP and C losses by ER (Woodwell and Whittaker, 1968) (equation 1.1).

$$
N E P=G P P-E R=(G P P-R a)-R h=N P P-R h
$$


In opposition to NEP, net ecosystem exchange (NEE) (equation 1.2) offers the atmospheric convention on the same flux, but with the opposite sign to NEP. The ecosystem is acting as a $\mathrm{CO}_{2}$ sink when NEP is positive and a $\mathrm{CO}_{2}$ source when NEP is negative.

$$
N E E=E R-G P P=-N E P
$$

Currently, several techniques exist for measuring the exchange of $\mathrm{CO}_{2}$ from an ecosystem to the atmosphere at various spatial scales. The technique that is commonly used is the eddy-covariance (EC) method, which directly measures the fluxes of $\mathrm{CO}_{2}$, water vapor, and energy from a land surface (see Box 1.1). Since the early 1990s, EC measurements have been conducted on all continents spanning much of the world's climate space and representative biomes (Fig. 1.2). The wide availability of these data has provided vital information about terrestrial $\mathrm{C}$ balances on a variety of ecosystems. The EC technique has also enabled the study of the ecosystems's responses to climate extremes (e.g. drought) and disturbance regimes (e.g. fire).

\section{Box 1.1: The EC method for the calculation of $\mathrm{CO}_{2}$ fluxes. Adapted from Curtis and Gough (2018).}

The EC method (Foken et al., 2012; Montgomery, 1948; Swinbank, 1951) provides a unique approach to directly observe the fluxes of $\mathrm{CO}_{2}$, water vapor, and energy from a land surface. The term 'eddy-covariance' is based on the principle that the vertical flux of $\mathrm{CO}_{2}$, water vapor, energy, and other atmospheric components within a turbulent boundary layer is proportional to the covariance of the vertical velocity and its concentration (Burba and Anderson, 2007). For instance, the $\mathrm{CO}_{2}$ flux $\left(\mathrm{Fc}\right.$ in $\left.\mu \mathrm{mol} \mathrm{m}{ }^{-2} \mathrm{~s}^{-1}\right)$ is calculated as the mean covariance between deviations in instantaneous deviation in vertical wind speed $(w /$ in $\mathrm{m}$ $\left.\mathrm{s}^{-1}\right)$ and $\mathrm{CO}_{2}$ concentrations in the air $\left(q^{\prime} c\right.$ in $\left.\mu \mathrm{mol} \mathrm{m}{ }^{-3}\right)$ multiplied by mean air density $\left(\rho_{a}\right)$ :

$$
F c=\overline{\rho_{a}} \cdot \overline{w^{\prime} q^{\prime} c}
$$

The EC technique measures the ecosystem response from short-term (half-hourly) to longterm (seasonal and interannual) variations and provides nearly continuous data. The processing chain of EC data, which includes data quality check, flux partitioning, and gap-filling, is done according to well-established methods (Lasslop et al., 2010; Papale et al., 2006; Reichstein et al., 2005).

\subsection{Disturbance, forest age, and the carbon cycle}

Forests cover about $30 \%$ of the terrestrial surface of our planet and store a large part of the terrestrial $\mathrm{C}$, indicating their fundamental role in terrestrial $\mathrm{C}$ dynamics (Pan et al., 2011b). However, forest disturbances can cause physical damage to vegetation properties, thereby affect 


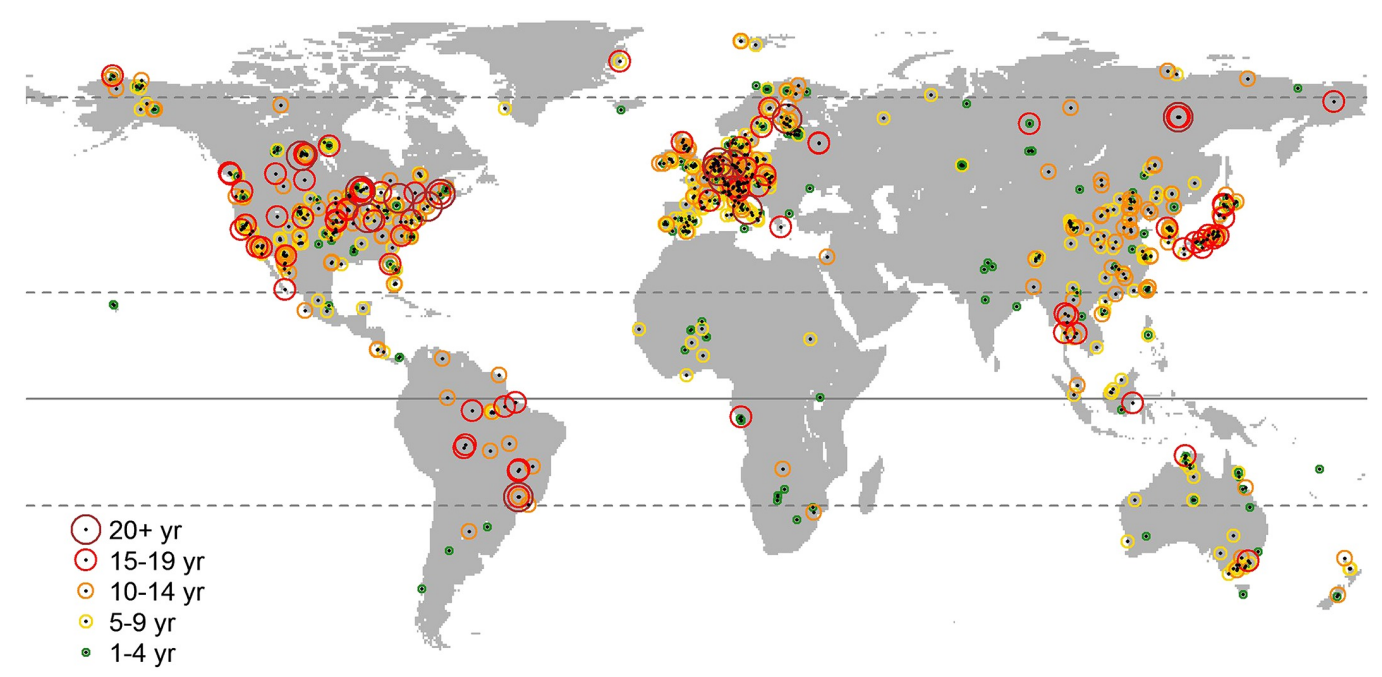

Figure 1.2: Map of active and historical FLUXNET tower sites used in the study. The color and size of the circle indicate the lengths of measurements as of December 2015. The solid and dashed lines denote equator, Tropic of Cancer/Capricorn, and the Arctic Circle, respectively. Figure adapted from Chu et al. (2017).

the balance of terrestrial $\mathrm{CO}_{2}$ exchange with the atmosphere by temporarily increasing respiration and reducing photosynthesis (Birdsey et al., 2006; Johnson and Curtis, 2001; Liu et al., 2011; Schimel, 2007; Williams et al., 2012; Woodbury et al., 2007). The changes in the strength of $\mathrm{C}$ uptake or release can alter the forest $\mathrm{C}$ balance by converting forest ecosystems from $\mathrm{C}$ sinks to sources (Amiro et al., 2010; Bowman et al., 2009; Ciais et al., 2014a; Moore et al., 2013). Disturbances arise from a range of agents such as drought, wildfire, logging, wind-throw, and insects (Law et al., 2003) and many of these disturbance processes are episodic and highly variable in space and time. Disturbed ecosystems cover a variable fractional area of the land and are intense sources of $\mathrm{CO}_{2}$ to the atmosphere until plant production recovers, leading to a gradual change in activity towards a $\mathrm{C}$ sink (Fig. 1.3). Under gradual climatic changes, there is a strong indication of modifications in magnitude and frequency of disturbance episodes (Turner, 2010).

Not only the time since a disturbance occurred can have different effects on the ecosystem's functioning, but also the types and intensities of disturbances (Law et al., 2003; Thornton et al., 2002). For instance, large forest disturbances (e.g. stand replacement, fire) entail a rapid release of $\mathrm{CO}_{2}$ which strongly affects the long-term $\mathrm{CO}_{2}$ sink's strengths (Zhou et al., 2013). Conversely, small-scale and partial disturbances (e.g. insect outbreaks) exert variable impacts both in space and time on the biosphere-atmosphere $\mathrm{CO}_{2}$ exchange (Zhou et al., 2013). However, such low-intensity disturbances affect leaf traits, and therefore can impact the $\mathrm{CO}_{2}$, energy, and water flux dynamics (Clark et al., 2010).

As previously discussed, the rate of forest $\mathrm{C}$ storage (or NEP) is driven by $\mathrm{C}$ assimilation (GPP) and $\mathrm{C}$ losses (ER). $\mathrm{C}$ accumulates in biomass and soils when GPP exceeds ER (i.e. positive 

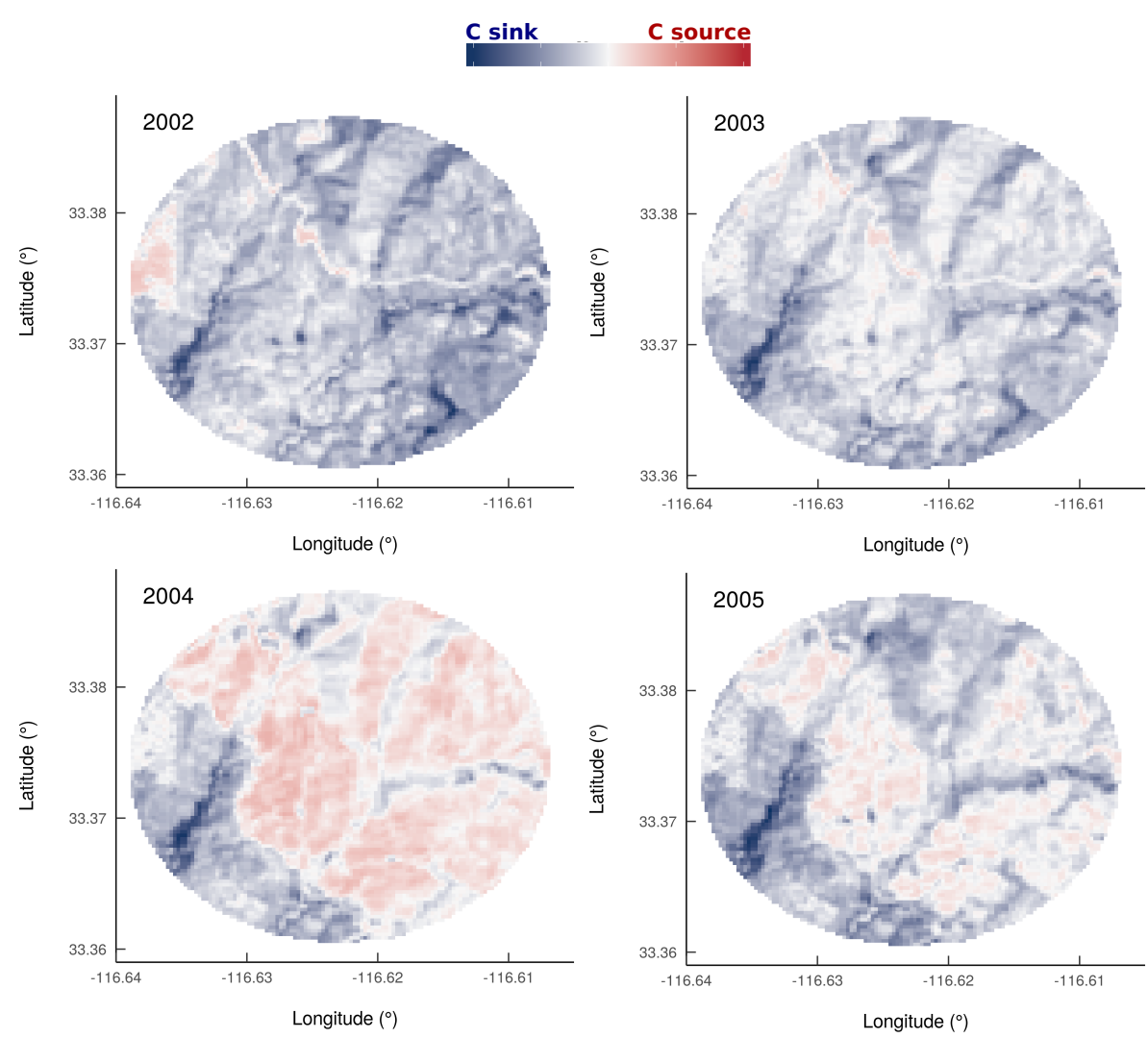

Figure 1.3: Illustration of the spatiotemporal impacts of disturbance on annual NEP. Within the presented footprint a fire occurred in July 2003 followed by a regrowth. This figure is derived from the approach presented in Chapter 4 using Landsat data.

NEP), whereas the opposite occurs when ER exceeds GPP (i.e. negative NEP). Both fluxes are contingent on environmental conditions such as temperature, soil moisture, and nutrient availability, as well as being sensitive to the intrinsic property of a stand, such as tree height, tissue nutrient content, and tree species. However, the responses of GPP and ER to these factors can be modified to varying degrees by disturbance and slowly change along a forest age or successional continuum (Irvine et al., 2005). The studies of Odum (1969) hypothesized the first theory to describe the ecosystem development in the absence of major disturbance. Since then, the proposed relationship between $\mathrm{C}$ exchange and forest age has been subsequently demonstrated and revised (Amiro et al., 2010; Buchmann and Schulze, 1999; Kashian et al., 2013; Pregitzer and Euskirchen, 2004; Ryan et al., 1997; Ryan et al., 2004; Tang et al., 2014), with particular emphasis on physiological differences between development stages. The NEP dynamic after disturbance (Fig. 1.4a) is generally characterized by a rapid increase during the early stages of forest succession, followed by a decline reaching either low $\mathrm{C}$ sources or sinks (Gough et al., 2016; Gough et al., 2008; Luyssaert et al., 2008; Pregitzer and Euskirchen, 2004). After a disturbance, $\mathrm{Rh}$ tends to increase because of an aboveground biomass (AGB) transfer to the litter C pool (Kurz et al., 2008), while GPP and Ra decrease due to a reduction in leaf area (Fig. 1.4b), resulting in a release of $\mathrm{CO}_{2}$ to the atmosphere (i.e. GPP $<\mathrm{Rh}+\mathrm{Ra}$ ). NEP 
recovers as both AGB and GPP increase whereas ER declines because of a loss of litter and soil $\mathrm{C}$ stocks (Fig. 1.4b). The resulting imbalance between GPP and ER persists until GPP comes in equilibrium with ER. Intensity and type of disturbances determine the rate and pathways of subsequent recovery (Meigs et al., 2009), resulting in highly variable spatiotemporal patterns of forest regrowth.

(a)

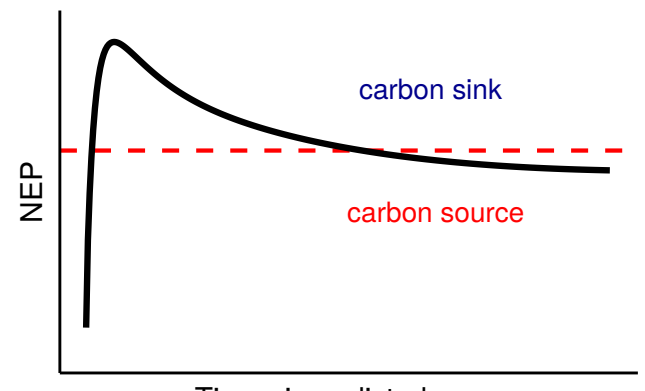

Time since disturbance (b)

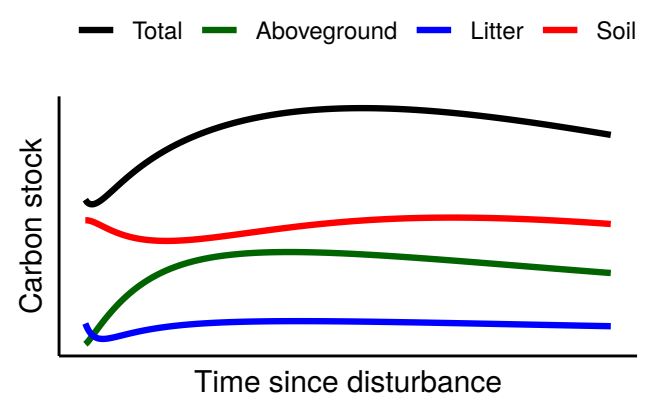

Figure 1.4: Theoretical ecological succession of NEP (a) and C stock dynamics (b) after forest disturbance.

Despite the fact that the forest $\mathrm{C}$ storage is to some extent controlled ecosystem recovery after disturbance (Buitenwerf et al., 2018; Sulla-Menashe et al., 2018), existing empirical models and current bottom-up spatiotemporal assessment of $\mathrm{CO}_{2}$ fluxes do not explicitly account for these effects. This is mainly due to the fact that the spatiotemporal $\mathrm{C}$ dynamics following a disturbance cannot be properly assessed at the global scale because of the lack of knowledge on global disturbance (Ciais et al., 2014a). On the one hand, our understanding of the temporal changes between $\mathrm{C}$ assimilation (GPP) and losses (ER) after a disturbance event across space and time is limited by the representativeness of the EC sites. The limited sample size is coupled with the sparsity of significant disturbances occurring during measurement periods. As such, both the spatial distribution of EC sites and the occurrence of disturbance events become limiting factors to characterize the forest age- $\mathrm{CO}_{2}$ flux dynamics. On the other hand, the variables used in the state-of-the-art bottom-up frameworks of net $\mathrm{CO}_{2}$ fluxes do not explicitly contain information related to disturbances (e.g. time since disturbance, recovery processes) (Jung et al., 2011; Tramontana et al., 2016). Instead, they assume that the information contained in vegetation and climate variables is sufficient to accurately estimate the responses of net $\mathrm{CO}_{2}$ flux estimates to disturbance regimes. All these limitations and assumptions challenge any attempts to explicitly account for the effects of disturbance history, therefore to produce reasonable global estimates of net $\mathrm{CO}_{2}$ fluxes from point observations.

Thereby, forest age, related to time since disturbance, can be seen as a useful surrogate in analyses of the impact of disturbance on the ability of forests to sequester and store C. Two applications can motivate exploring the control of forest age on net $\mathrm{CO}_{2}$ fluxes. First, it remains unclear what the quantitative role of forest age (or time since disturbance) in controlling spatiotemporal variations of net $\mathrm{CO}_{2}$ fluxes is. As such, this raises the question of how to best represent the 
effect of forest age in empirical models and how it interacts with additional model terms. Second, the recent advances in describing the geographical distribution of forest demography globally (Huang et al., 2010; Kennedy et al., 2010; Poulter et al., 2019) provide opportunities to consider forest age and disturbance history in data-driven upscaled products. Incorporating forest age into terrestrial biosphere modeling offers a starting point to characterize disturbance history, thereby our understanding of the vegetation C sink estimates (Pugh et al., 2019).

\subsection{Climate and vegetation ecological memory effects on the carbon cycle}

Similarly to forest demography, the ecological memory effects (also called lag effects) of both vegetation and climate contribute substantially to the $\mathrm{CO}_{2}$ flux variability (Monger et al., 2015; Seidl et al., 2014). Ecological memory effects can be defined as the ability of the past temporal trajectories of climate and vegetation to influence the current structure and functioning of ecosystems (Ogle et al., 2015; Peterson, 2002). In other words, vegetation productivity is not only driven by contemporary environmental conditions, but also by antecedent conditions that are not directly reflected on proxies describing present vegetation's state (e.g. vegetation greenness). As suggested by Frank et al., 2015, the ecosystems are not only responding to direct and indirect concurrent mechanisms but also to direct and indirect lag effects of vegetation and climate fluctuations. For instance, the direct effects can be related to a decrease in productivity during a drought event, while indirect concurrent effects can result in biomass loss from fire facilitated by an ongoing drought. A drought event can further negatively or positively impact the productivity of an ecosystem from months to years following this event, that is direct lag effects. A common example of indirect lag effects is the occurrence of insect outbreaks that is facilitated by past disturbance events (e.g. drought causes tree mortality and dead wood accumulation, which further contribute to insect outbreaks).

In recent years, the concurrent effects of vegetation and climate on biosphere-atmosphere $\mathrm{CO}_{2}$ fluxes have been well observed and described across ecosystems. For instance, extreme temperatures (e.g. heat waves, frost) or unusual warming events have variable effects on the response patterns of photosynthesis and ecosystem respiration to climate extremes. These events can damage a plant, ultimately changing the growth and development of the plant (Larcher, 2003; Lobell et al., 2012; Niu et al., 2014), or can even perturb the timing of seasonal plant development (e.g. earlier onset of the growing season due to warm late winters) (Marino et al., 2011). Similarly, evidence with regard to the vegetation and climate's ecological memory effects has been provided. For instance, spring temperature seems to have variable memory effects on subsequent summer and autumn ecosystem productivity (Buermann et al., 2018), while Aubinet et al. (2018) found a dependency of the current ecosystem functioning to previous year water limitation. This evidence points at the need to understand the sensitivity of global ecosystems to past-time climate variability and disturbances. Yet, the scientific community has had less 
success in explaining ecological memory effects compared to concurrent effects mainly due to the complex non-linear responses of ecosystems to past climate extreme and disturbance events. For example, the observed behavior of a forest ecosystem (e.g. during the period EC measurements) can depend on a series of complex mechanisms related to the temporal context pre- and post-disturbance (Fig. 1.5). In fact, depending on the state of the forest pre-disturbance, the magnitude of the disturbance, the recovery capacity of the forest, its state post-disturbance, and the environmental conditions the resulting biosphere-atmosphere $\mathrm{CO}_{2}$ exchange in such a disturbed ecosystem can be substantially different. This implies that it is relevant to incorporate these short- to long-term processes to diagnose the $\mathrm{C}$ dynamics in terrestrial ecosystems, albeit it is currently challenging. A common practice to incorporate these dynamic effects is to derive hand-designed variables in machine learning methods, such as lag variables (Tramontana et al., 2016). However, this practice is, most of the time, being applied for only one variable, therefore overlooks the interactive ecological memory effects between variables (Reichstein et al., 2019) limiting our capacity to characterize the non-linear feedback between extreme events and ecosystem responses. Consequently, the direct and indirect ecological memory effects of climate extremes and past disturbances in the trajectories of terrestrial ecosystems' $\mathrm{C}$ dynamics are still poorly understood (Thom et al., 2018).

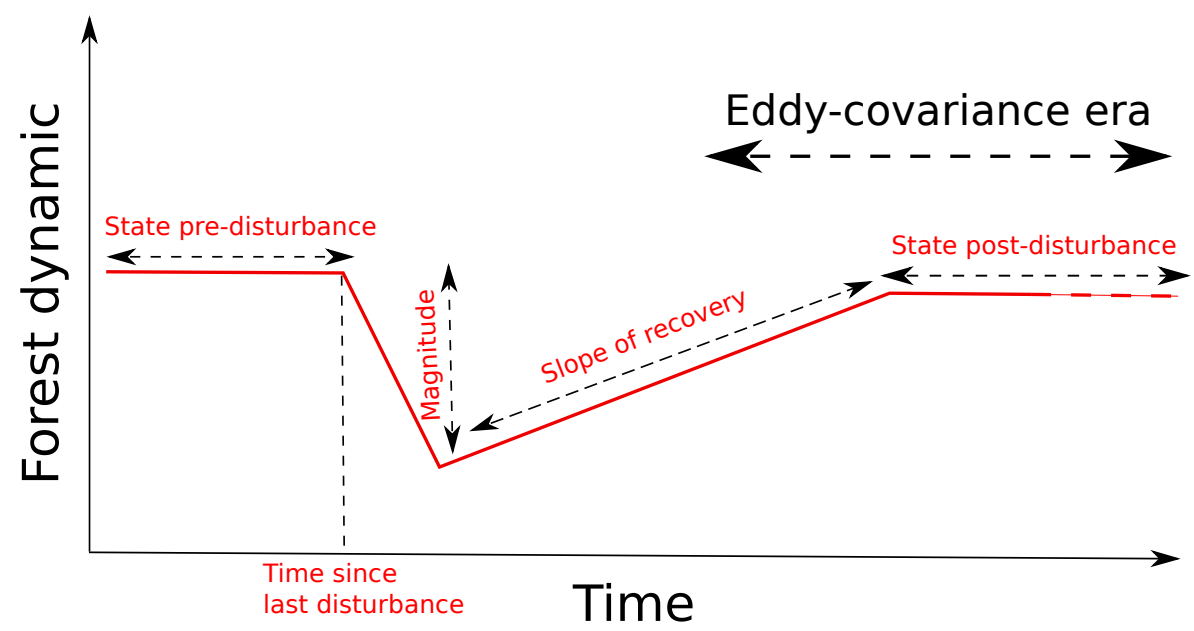

Figure 1.5: Schematic diagram illustrating the temporal forest dynamics before and after a disturbance event.

The question is then how to dynamically incorporate the effects of ecosystem history (i.e. recent and past vegetation and climate's dynamics) on $\mathrm{CO}_{2}$ fluxes into data-driven models. The answer to this question is two fold: plethora of Earth system data and dynamic deep learning (DL) methods. The availability of free, high quality, and long-term remote sensing products (e.g. Landsat and MODerate-resolution Imaging Spectroradiometer (MODIS) data) opens new avenues to represent long-term vegetation dynamics, disturbance regimes, and forest recovery dynamics at fine spatial (e.g. 30 meters for Landsat data) and temporal resolutions (e.g. daily resolution for MODIS data). Remote sensing products, such as Landsat and MODIS, can provide relevant knowledge related to vegetation greenness (e.g. normalised difference 
vegetation index (NDVI)) or to the physiological status of vegetation (e.g. normalised difference moisture index (NDMI)). As such, remote sensing of the biosphere from space with a short return interval to identical locations and nearly global coverage offers promising perspectives to, for instance, detect extreme anomalies in the biosphere in a consistent way. In the past decade, remote sensing data have been applied to a wide range of environmental monitoring tasks such as (i) time series analysis for monitoring recovery dynamics and anomaly detection (DeVries et al., 2015; Kennedy et al., 2010), or (ii) regression analysis in geosciences (e.g. predictions of the spatial, seasonal, and interannual variability of the $\mathrm{CO}_{2}$ flux variability) (Jung et al., 2011; Tramontana et al., 2016). Similarly, climate model simulation outputs spanning from the 1900s until now provide relevant information on long-term climate fluctuations from hourly to inter-annual scales (e.g. ERA and CRUNCEP climate data). In this context, the integration of long-term climate and vegetation data have proved to be remarkable sources of information for assessing long-term climate-vegetation feedback and the $\mathrm{C}$ cycle. Furthermore, the recent development of dynamic DL methods, such as the recurrent neural network (RNN) and Long-Short-Term Memory (LSTM) models (Hochreiter and Schmidhuber, 1997), enables the extraction of vegetation and climate temporal features that are key to understand ecosystem responses to climate extremes and disturbances. So far, such a dynamic approach had success in sequence learning (e.g. speech recognition) and land cover classification (Rußwurm and Körner, 2017), but application to $\mathrm{CO}_{2}$ flux predictions is still in its early stage (Reichstein et al., 2018). Conceptually, integrating long-term climate and vegetation data into temporally dynamic statistical methods (e.g. LSTM models) may enable the consideration of climate and vegetation's ecological memory effects when modelling and understanding the $\mathrm{C}$ cycle.

\subsection{Principal research objectives}

This thesis aims to provide a more realistic view on the relevance of ecosystem history to the biosphere-atmosphere $\mathrm{CO}_{2}$ exchange at the global scale. This is done from an empirical perspective by using different sources of data (i.e. eddy-covariance, ancillary, remote sensing, and climate observations) and a series of statistical models (i.e. non-dynamic and dynamic statistical models). More specifically the following research questions are investigated and answered:

1. What is the contribution of forest age to the terrestrial net $\mathrm{C}$ fluxes? Can forest age be explicitly accounted for when upscaling net $\mathrm{CO}_{2}$ fluxes?;

2. How important is vegetation and climate's ecological memory effects on terrestrial $\mathrm{CO}_{2}$ fluxes?; and

3. What is the magnitude and geographical distribution of climate and vegetation's ecological memory effects on terrestrial $\mathrm{CO}_{2}$ fluxes globally? What are the implications of ecological memory effects in net $\mathrm{CO}_{2}$ flux's responses during climate extreme events? 


\subsection{Thesis Overview}

This thesis consists of six chapters designed to investigate and answer the above mentioned questions, ultimately evaluating the influence of ecosystem history to the biosphere-atmosphere $\mathrm{CO}_{2}$ exchange.

In Chapter 1, I present this general introduction, contextualizing the terrestrial ecosystems and the global $\mathrm{C}$ cycle as well as explaining why the effects of ecosystem history are of relevance to understand the biosphere-atmosphere $\mathrm{CO}_{2}$ exchange.

In Chapter 2, I briefly present the current state of knowledge with regard to the control of forest age and disturbance history on the forest $\mathrm{C}$ balance. This led to the implementation of a new empircial model, which does not only account for the relations between environmental conditions and forest NEP but also for the effects of forest age. The implications of considering forest age in such a modeling excercise are then analyzed and discussed. This chapter is based on a published journal article (Besnard et al., 2018).

In Chapter 3, we follow-up on the work of Chapter 2 by incorporating the effects of forest age in the production of forest NEP's global estimates from point observations. In this chapter, we introduce a new forest age map and provide new estimates of both global forest NEP and NBP, which are further compared with independent forest inventories' estimates. These new forest NEP gridded products also provide a new assessment of the location of the forest $\mathrm{C}$ sinks and sources globally.

In Chapter 4, I apply a DL method (i.e. LSTM model) that captures the temporal dynamic of vegetation and climate, thus presenting a novel application of DL methods in Earth system science. I test different model training procedures in order to depict the relevance of capturing vegetation and climate's ecological memory effects when modelling NEE in forest ecosystems. Ultimately, I show that a model that considers climate and vegetation's ecological memory effects leads to a significant improvement of predicted forest NEE compared to non-dynamic models (i.e. Random Forest). We also emphasise the importance of the vegetation seasonal context in controlling NEE spatiotemporal variability in forest ecosystems. This chapter is based on a published journal article (Besnard et al., 2019a).

In Chapter 5, I examine the ecological memory effects of climate and vegetation on biosphereatmosphere $\mathrm{CO}_{2}$ exchange at the global scale. Based on the method implemented in Chapter 4, I present new insights on the magnitude and the geographical distribution of vegetation and climate's ecological memory effects across scales (i.e. seasonal cycle, seasonal anomalies, interannual, and across space). I analyse and discuss these findings by correlating such observed spatial patterns with ecosystem characteritics and climatic conditions. I also analyze the 2018 European heatwave event for understanding the impacts of vegetation and climate's ecological memory effects on biosphere-atmosphere $\mathrm{CO}_{2}$ exchange during such a climate extreme event. 
In Chapter 6, I synthetize the main findings of this thesis and discuss existing issues found in the previous chapters. I conclude by suggesting possible avenues that could be explored in further research questions in order to overcome existing limitations in the approaches proposed in my thesis. I also discuss the potential of new modeling frameworks in Earth system science (e.g. transfer learning, hybrid modeling) and reflect on the feasibility of implementing operational systems for $\mathrm{CO}_{2}$ flux monitoring. 


\section{Chapter 2}

\section{Quantifying the effect of forest age in annual net forest carbon balance}

This chapter is based on:

S. Besnard, N. Carvalhais, M. A. Arain, A. Black, S. d. Bruin, N. Buchmann, A. Cescatti, J. Chen, J. G. P. W. Clevers, A. R. Desai, C. M. Gough, K. Havrankova, M. Herold, L. Hörtnagl, M. Jung, A. Knohl, B. Kruijt, L. Krupkova, B. E. Law, A. Lindroth, A. Noormets, O. Roupsard, R. Steinbrecher, A. Varlagin, C. Vincke, and M. Reichstein (2018). "Quantifying the effect of forest age in annual net forest carbon balance". Environmental Research Letters 13.12, 124018 


\begin{abstract}
Forests dominate carbon (C) exchanges between the terrestrial biosphere and the atmosphere on land. In the long term, the net $\mathrm{C}$ flux between forests and the atmosphere has been significantly impacted by changes in forest cover area and structure due to ecological disturbances and management activities. Current empirical approaches for estimating net ecosystem production (NEP) rarely consider forest age as a predictor, which represents variation in physiological processes that can respond differently to environmental drivers, and regrowth following disturbance. Here, we conduct an observational synthesis to empirically determine to what extent climate, soil properties, nitrogen deposition, forest age and management influence the spatiotemporal variability of annual forest NEP across 126 forest eddy-covariance flux sites worldwide. The empirical models explained up to $62 \%$ and $71 \%$ of spatiotemporal and across-site variability of annual NEP, respectively. An investigation of model structures revealed that forest age was a dominant factor of NEP spatiotemporal variability in both space and time at the global scale as compared to abiotic factors such as nutrient availability, soil characteristics, and climate. These findings emphasize the importance of forest age in quantifying spatiotemporal variation in annual NEP using empirical approaches.
\end{abstract}




\subsection{Introduction}

Forests cover about $30 \%$ of the Earth's terrestrial surface and store around $90 \%$ of terrestrial vegetation carbon (C) (Canadell et al., 2000; Gower, 2003; Le Quéré et al., 2018), indicating their fundamental role in terrestrial C dynamics (Beer et al., 2010; Bonan, 2008; Carvalhais et al., 2014; Hicke et al., 2012; Pan et al., 2011a). However, the functioning of forest ecosystems is likely to be altered by changing climate (Ciais et al., 2005; Reichstein et al., 2013; Xiao et al., 2009; Zhao and Running, 2010), ecological disturbances (Amiro et al., 2010; Bowman et al., 2009; Chambers et al., 2007), and management (Naudts et al., 2016; Noormets et al., 2015). Therefore, it is important to characterize current and future forest net ecosystem production (NEP) for regional to country-level assessments, and to evaluate mitigation strategies that minimize carbon dioxide $\left(\mathrm{CO}_{2}\right)$ emissions to the atmosphere (Becknell et al., 2015; Law et al., 2018; Trumbore et al., 2015).

The overall NEP dynamic at a given site emerges from combined responses to factors that control both gross primary productivity (GPP) and ecosystem respiration (ER) (NEP=GPP-ER). At the ecosystem level, the forest NEP patterns following stand-replacing disturbance are mostly controlled by the time-varying dominance between autotrophic and catabolic processes. After disturbance, heterotrophic respiration ( $\mathrm{Rh}$ ) generally tends to increase because of an aboveground biomass transfer to the litter and soil organic matter C pools (Harmon et al., 2011; Kurz et al., 2008; Law et al., 2003; Lindauer et al., 2014; Noormets et al., 2012; Paul-Limoges et al., 2015), while GPP collapses due to an instantaneous reduction in leaf area, resulting in a net release of $\mathrm{CO}_{2}$ to the atmosphere. On the one hand, a shift from $\mathrm{C}$ source to $\mathrm{C}$ sink occurs as canopy development supports GPP and net $\mathrm{C}$ accumulation in plants increase. On the other hand, $\mathrm{Rh}$ and ER decline due to a reduction in litterfall and substrate availability through decomposition. The resulting imbalance between GPP and ER persists until vegetation and soil C pools increase up to the point when ER comes into equilibrium with GPP (Lindroth et al., 2008; Luyssaert et al., 2008; Schwarz et al., 2004; Tang et al., 2014).

Several approaches used for assessing forest NEP include micro-meteorological and biometric techniques, process-based models, and/or satellite data. However, annual regional C stock assessments that account for age-related physiology, regrowth, and soil processes following disturbance are challenging due to lack of information in disturbance history or management practices (Zscheischler et al., 2017). Flux tower networks (e.g. FLUXNET) provide the annual net uptake of $\mathrm{CO}_{2}$ from the atmosphere (i.e. NEP) that can be used to calibrate empirical models for mapping annual NEP at regional scales. However, current empirical upscaling exercises (Jung et al., 2011; Tramontana et al., 2016) do not directly include proxies that allow the dynamics of $\mathrm{C}$ fluxes with age to emerge, therefore it is not clear how well the aforementioned data-driven models captured such dynamics. Thereby, empirical estimation of annual NEP that explicitly accounts for disturbance and forest age effects are of relevance for regional C stock studies (Ciais et al., 2014a). 
Despite the recognized effects of forest age in controlling spatial and interannual variability of NEP, there is still debate about the quantitative role of forest age in the empirical annual forest $\mathrm{C}$ estimates. In fact, the most recent observation-based synthesis studies tackling NEP spatiotemporal variability and its drivers reached diverging conclusions on the importance of forest age. While some authors have shown that forest age is a key factor controlling forest $\mathrm{C}$ balance (Chen et al., 2003; Chen et al., 2002; Coursolle et al., 2012; Gao et al., 2016; Yu et al., 2014), others have indicated that spatial and interannual variability of NEP is mainly controlled by nutrients availability and soil properties (Bhatti et al., 2002; Fernández-Martínez et al., 2014; Janssens et al., 2010; Vicca et al., 2012) or climate conditions (Amiro et al., 2006; Coursolle et al., 2006; Thornton et al., 2002), although several authors report that the $\mathrm{C}$ budget in forest ecosystems is less sensitive to climatic conditions than expected in certain regions (Law et al., 2002; Reichstein et al., 2007b; Yi et al., 2010).

Given the fundamental understanding of the role of forest age in NEP and the contrasting results from previous meta-analyses, we revisited the importance of forest age to the spatial and temporal variability in NEP based on a more up-to-date, larger, and higher quality eddycovariance (EC) dataset including 126 forest ecosystem sites. We further expanded previous observation-based syntheses by exploring non-linear empirical model formulations to incorporate forest developmental stage and environmental factors for calculating realistic NEP spatiotemporal variability. Such a model can eventually be used to estimate NEP at a global scale and infer likely limits to NEP variation and the future forest $\mathrm{C}$ sink as forests age.

\subsection{Methods}

\subsubsection{Datasets}

We used a global dataset of $126 \mathrm{EC}$ forest sites ranging from 0 to $\geq 300$-year-old stands (Table 2.A1 and Fig. 2.A1). The sites were part of both version 2 of the LaThuile FLUXNET and the FLUXNET2015 datasets (https://fluxnet.fluxdata.org) of the FLUXNET network (Baldocchi, 2008; Baldocchi et al., 2001). Five vegetation types were considered in the study, including 76 evergreen forests, 27 deciduous forests, 11 mixed forests, 7 shrublands, 3 savannas, and 2 wetlands.

We aggregated daily NEP, GPP, ER and the associated uncertainties into annual sums (i.e. siteyears) and computed an annual-average from all available years per site (i.e. site-average) (see supplementary information for details on EC data processing). One relevant aspect to consider is that the observation-derived GPP is determined via the measured night-time NEP (Reichstein et al., 2005). This challenges the statistical independence of both variables, therefore risking a spurious correlation between GPP and NEP at annual scales (see supplementary materials). To avoid any spurious relationship between NEP and GPP, we used a proxy for GPP, i.e. GPP', 
which was determined as the ratio between latent heat flux (LE) and the square root of vapor pressure deficit (VPD) $\left(G P P^{\prime}=\frac{L E}{\sqrt{V P D}}\right)$ (see supplementary materials for more details and Fig. 2.A3) as based on physiological principles of the coupling between the $\mathrm{C}$ and water cycles at the leaf level (Chen et al., 2002; Katul et al., 2009; Katul et al., 2010). While leaf area index (LAI) and fraction of absorbed photosynthetically active radiation (fPAR) could have been used as proxies for GPP, GPP' is the integrated response of phenology and physiology, therefore a direct metric of primary productivity, while the former are mostly phenology driven.

We considered forest age as the time since forest establishment or as the time since the occurrence of the last stand-replacing disturbance (see supplementary information for more details on the definition of forest age). Sites were selected based on the availability of information about forest age, disturbance history, and management that would allow for an appropriate definition of a meaningful site stand age. These included a range of young and old growth forest sites (Fig. 2.A2) that were established after complete, nearly complete or substantial removal of forest vegetation (e.g. harvest, fire, wind-throw, insect outbreaks), followed by reforestation/succession/afforestation activities within the flux tower footprint. We did not consider sites with ambiguous historical information or those that had experienced only low to partial intensity disturbances, which would not allow the determination of whole stand forest age. For uneven-aged stands, we followed Spies, Franklin, et al., 1991 and estimated the age of a stand as the age of the largest $10 \%$ of trees. Undisturbed old-growth forests where age information was available were also included. The information for each site was obtained from the literature, provided by the site principal investigators or from the Biological, Ancillary, Disturbance and Metadata database (Table 2.A1). In general, the wide span in stand age among sites and the multi-year record of observations per site permit evaluating the effect of age on both the mean and the interannual variability in NEP.

In addition to the $\mathrm{CO}_{2}$ and water fluxes, we also obtained the following variables as statistical covariates for model development for each EC site:

(i) local microclimatic variables from in situ observations (i.e. air temperature $\left(\mathrm{T}_{\text {air }}\right)$, precipitation (Precip), and global radiation $(\mathrm{Rg}))$;

(ii) information on nutrient availability (NA) divided into three classes: low NA ( $n=67$ sites); medium NA ( $\mathrm{n}=41$ sites); and high NA ( $\mathrm{n}=18$ sites) (based on Fernández-Martínez et al. (2014) and/or expert knowledge, Table 2.A1);

(iii) additional information on soil texture up to 2-m depth from the SoilGrids $1 \mathrm{~km}$ dataset (Hengl et al., 2014);

(iv) information on forest management based on Campioli et al. (2015), Luyssaert et al. (2008) datasets, and indications from the principal investigators (PIs) (managed forests $n=44$ sites; and unmanaged forests $n=81$ sites). Managed sites were dominated by human activity while unmanaged sites were undisturbed or experienced low human impacts; 
(v) gridded monthly temperature and precipitation observations from the Climate Research Unit (CRU) (http: //www . cru .uea .ac .uk) (Harris et al., 2014) to determine long-term linear climate trends and anomalies; and

(vi) local total atmospheric nitrogen deposition $\left(\mathrm{N}_{\text {deposition }}\right)$ from in situ observations. We collected $\mathrm{N}_{\text {deposition }}$ estimates from the gridded emissions dataset (Wang et al., 2017) at $0.5^{\circ}$ x $0.5^{\circ}$ resolution when they were not available at site level.

\subsubsection{Net ecosystem production model development}

The development of an NEP statistical model principally aimed to provide a data-driven estimate of the several factors that control the temporal and spatial variability in NEP, and further to estimate the relative contribution of the different predictive variables - especially age and GPP' - to variation in NEP. To do so, we used the aforementioned statistical covariates (i.e. GPP', microclimatic data, NA, soil texture, $\mathrm{N}_{\text {deposition }}$, forest age, and forest management) to train and evaluate the ability of a Random Forest (RF) (Breiman, 2001) algorithm to explain NEP variability.

$\mathrm{GPP}^{\prime}$ and ER are co-determinants of NEP, therefore both were initially compared to represent site level NEP. However, given the higher correlation of NEP with GPP' compared to the relationship with ER (Fig. 2.A3), and the aforementioned statistical dependence between NEP and the gross $\mathrm{C}$ fluxes (i.e. ER and GPP), we chose to discard ER in the statistical analysis.

The role of forest age as an explanatory variable of NEP was additionally evaluated with a published non-linear model (from now on identified as $g($ age)) to represent the NEP-age relationship (equation 2.1 and Fig. 2.1).

$$
N E P=o f f s e t+a \cdot\left(1-e^{b \cdot A g e}\right)(\text { hereafter } \mathbf{g}(\text { age }))
$$

adapted from Amiro et al., 2010, where the model parameters a $\left(\mathrm{gC} \mathrm{m}^{-2} \mathrm{y}^{-1}\right), \mathrm{b}$ (unitless), and offset $\left(\mathrm{gC} \mathrm{m}^{-2} \mathrm{y}^{-1}\right)$ were estimated. $\mathrm{g}($ age $)$ was expressed in $\mathrm{gC} \mathrm{m}^{-2} \mathrm{y}^{-1}$.

Although the NEP-age model (equation 2.1) was originally developed to represent the temporal patterns of annual GPP-to-ER ratio in forest chronosequences, here we used it to describe the dependency of spatiotemporal NEP variability on forest age. The selection of the Amiro et al. (2010) model to describe NEP spatial temporal dynamics assumed that the age-related patterns in GPP/ER were qualitatively similar to NEP, and was supported by a comparison to two other different empirical models presented in the literature (Coursolle et al., 2012; Tang et al., 2014). These two models were also tested but showed poorer model performance than the Amiro et al., 2010 model in the multivariate analysis (Table 2.A2). 


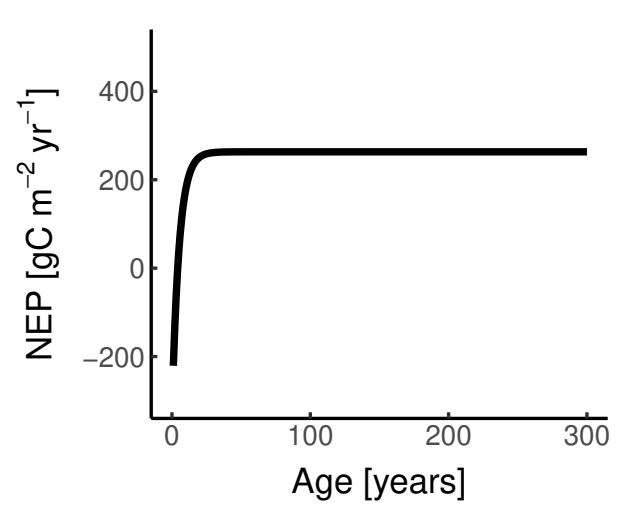

Figure 2.1: NEP-age dynamics for the Amiro et al. (2010) stand age model. a $=587.74 \mathrm{gC} \mathrm{m}^{-2}$ $\mathrm{y}^{-1}, \mathrm{~b}=-0.19$, and offset $=-324.54 \mathrm{gC} \mathrm{m}^{-2} \mathrm{y}^{-1}$

\subsubsection{Experiment design}

Estimation of forest age model parameters: The model parameters of the forest-age model (equation 2.1) were first estimated in a leave-one-site-out cross-validation mode for the entire dataset based on a generalized non-linear least squares (gnls) model using R software (Team, 2015). To account for temporal auto-correlation of the observations, we combined the gnls model with an auto-regression moving average model. We minimized the sum of squared residuals weighted for the uncertainty of the observations (Richardson and Hollinger, 2005). The standard errors of model parameters were estimated using a bootstrapping algorithm $(\mathrm{N}=500$ random resamplings). The model output (i.e. $g($ age )) was further included as a covariate in the training of the RF algorithms as a non-linear formulation of age effects on spatiotemporal variability of NEP.

RF algorithm and variable selection: We tested and assessed a RF algorithm using the caret $\mathrm{R}$ package (Kuhn, 2008). This is a non-parametric technique, which makes no assumption about the residuals of the model. A priori, we used a regression algorithm (i.e. the Boruta algorithm (Kursa and Rudnicki, 2010)), to determine the best set of predictive variables for NEP among the aforementioned variables. The Boruta method relies on an RF method and determines relevance of each variable by comparing the relevance of the original predictors to that of the randomized variables. It iteratively removes the features that are shown by a statistical test to be less relevant than random probes. We decided to keep the five best variables to improve the accuracy of predictions.

Model performance and model sensitivities: The performance of the statistical model was evaluated by directly comparing model estimates with observed values of NEP for each siteaverage or site-year in a leave-one-site-out cross-validation mode. In other words, we excluded one site at a time in every training set to predict the mean NEP (site-average) or the annual variations in NEP (site-year) at the excluded site. The statistics used to analyze the results included the coefficient of determination $\left(\mathrm{R}^{2}\right)$, Nash-Sutcliffe model efficiency (NSE), root 
mean square error (RMSE), and mean absolute error (MAE) (Omlin and Reichert, 1999). To quantify the importance of each predictive variable, we performed a model sensitivity analysis by removing a predictor from each regression analysis, then refitted and re-assessed the model without the variable left out. For the site-average analysis, the statistical model was trained using the site-years dataset. The same predictions were further averaged per site and compared to the site-average observations.

\subsection{Results}

\subsubsection{Age-dependent forest carbon dynamics}

The statistical dependence of NEP on forest age supported the non-linear NEP-age relationship (Fig. 2.2 and equation 2.1) in that NEP increased rapidly with age followed by stabilization with forests aging (Fig. 2.2). This finding reflected the expected age-related change in the size and the dynamics of the C pools (Gray et al., 2016; Noormets et al., 2015; Woodall et al., 2015; Zhang et al., 2015a). However, NEP-age dynamics appeared to depend of climatic conditions, because biomass accumulation rates of regrowing forests vary with climate (Anderson-Teixeira et al., 2013; Chazdon et al., 2016). This covariation partly explained the differences of the timing when a maximum NEP was reached and then gradually decreased as forest ages among different environmental conditions and the substantial scatter of observations around the model response due to inter-site variability.

The low correlation coefficient between NEP and age (Table 2.1) $\left(\mathrm{R}^{2}=0.09\right.$ and 0.2 for siteyears and site-average, respectively) could be attributed to the substantial contribution of other environmental factors to the spatial and temporal variability of NEP (Fig. 2.2). A model based on forest age alone was unable to capture such dependencies and warranted the need to include additional factors in a regression analysis. Although the regression did not show a substantial correlation, the fitted function showed a strong statistical significance, mostly because of the initial curve inflection attributed to the large effect of disturbances on the NEP fluxes in the first years of a recovery process (Amiro et al., 2010; Coursolle et al., 2012; Tang et al., 2014).

Table 2.1: Model parameter estimates of univariate relationships between NEP and forest age. The standard errors of the parameter estimates are shown in brackets. Statistics of the forest age model are also shown. $\mathrm{R}^{2}=$ coefficient of determination; $\mathrm{MAE}=$ mean absolute error; $\mathrm{AIC}=$ Akaike Information Criterion; total $n=716$ for all years per site and $n=126$ for average site.

\begin{tabular}{l|ccc|ccc} 
& \multicolumn{3}{|c|}{ Parameters } & \multicolumn{3}{|c}{ Model performance } \\
& Offset $\left[\mathrm{gC} \mathrm{m}^{-2} \mathrm{y}^{-1}\right]$ & $\mathrm{a}\left[\mathrm{gC} \mathrm{m} \mathrm{m}^{-2} \mathrm{y}^{-1}\right]$ & $\mathrm{b}$ & $\mathrm{R}^{2}$ & MAE & AIC \\
\hline All years per site & $-324.7(106.8)^{* * *}$ & $587.7(106.9)^{* * *}$ & $-0.2(0.07)^{* * * *}$ & 0.09 & 224.2 & 9226.4 \\
Average per site & $-482.6(366.5)$ & $760.8(362.7)^{* * *}$ & $-0.3(0.2)$ & 0.2 & 209.7 & 1753.2
\end{tabular}


(a)

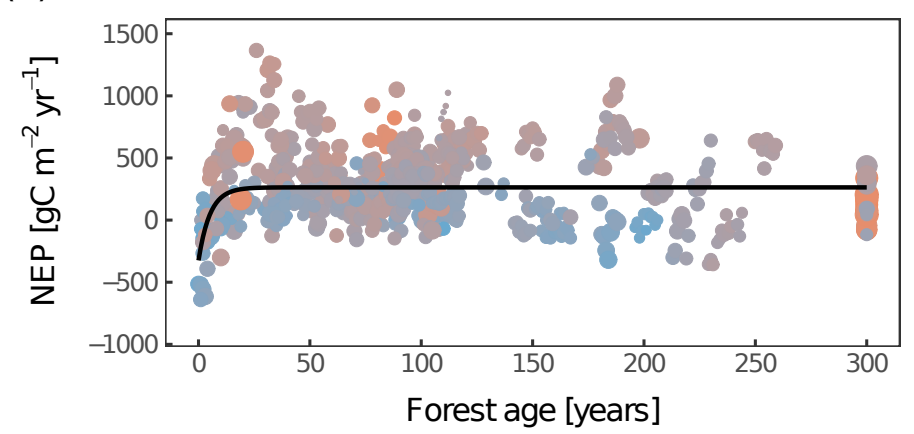

(b)

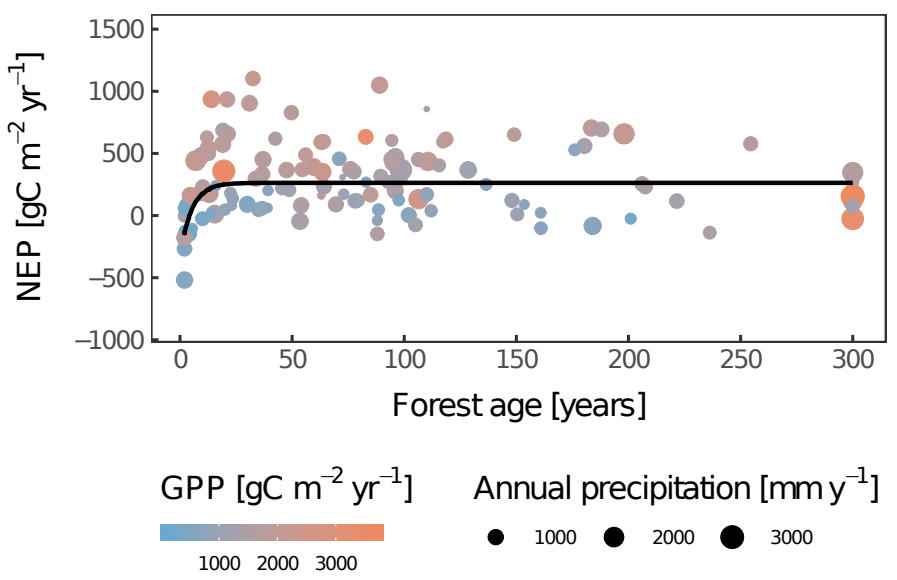

Figure 2.2: Relationship between NEP and forest age for (a) site-years and (b) site-average. The dots indicate the measurements. The lines indicate modeled NEP using the median of the parameter estimates from Amiro et al. (2010) function.

\subsubsection{Random forest algorithm performance and model sensitivity}

Based on the aforementioned feature selection criterion (Table 2.A3), the RF algorithm accounted for the effects of forest age (i.e. age and $\mathrm{g}(\mathrm{age})$ ), $\mathrm{GPP}^{\prime}, \mathrm{T}_{\text {air }}$, and $\mathrm{N}_{\text {deposition }}$ ). Both site-years and site-average variability were well captured by the different RF models (NSE $=0.62$ for site-years and NSE $=0.71$ for site-average) (Fig. 2.3), suggesting that the structure of the models was suitable for reproducing the spatiotemporal patterns of annual NEP. In addition, for both scenarios (i.e. site-years and site-average), we found that a model including only forest age and $\mathrm{GPP}^{\prime}$ as predictive variables (i.e. $\mathrm{NEP}=\mathrm{f}\left(\right.$ age, $\mathrm{g}($ age $\left.), \mathrm{GPP}^{\prime}\right)$ had a good predictive capacity for both site-years and site-average (NSE $=0.60$ for site-years and NSE $=0.67$ for site-average) Although we depicted some high values in the residuals across-site (maximum $=454.4 \mathrm{gC} \mathrm{m}^{-2}$ $\mathrm{y}^{-1}$; minimum $=-537.4 \mathrm{gC} \mathrm{m}^{-2} \mathrm{y}^{-1}$ ), we found no significant patterns of residuals against covariates (Fig. 2.A4).

Model sensitivity tests whereby predictors were sequentially removed (Table 2.2) supported the importance of forest age for explaining NEP variability. Whenever we removed forest age from 
(a)

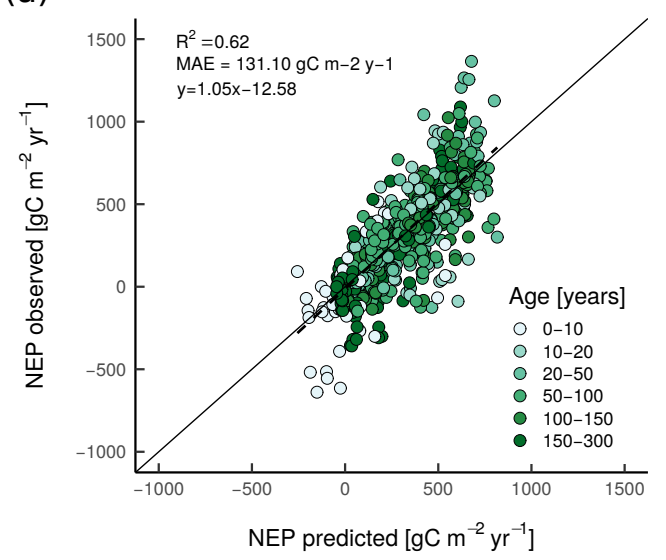

(b)

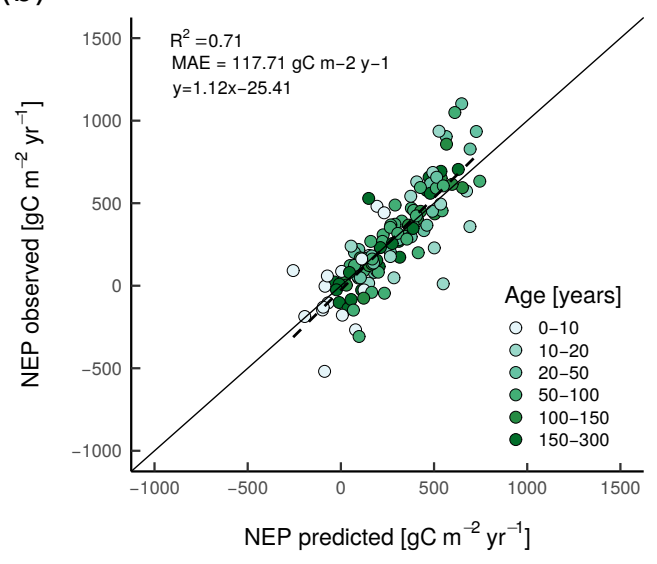

Figure 2.3: Cross-validated comparison of predicted vs. observed NEP estimates using the best model set-up for (a) site-years and (b) site-average. The scatterplots of the observed vs. modelled annual sums of NEP were grouped by age classes. NEP $=\mathrm{f}\left(\right.$ age, $\left.\mathrm{g}(\mathrm{age}), \mathrm{GPP}^{\prime}, \mathrm{T}_{\text {air }}, \mathrm{N}_{\text {deposition }}\right)$.

the RF models, model performance decreased, while there were only small changes in model performance when removing either $\mathrm{GPP}^{\prime}, \mathrm{T}_{\text {air }}$ or $\mathrm{N}_{\text {deposition }}$.

Table 2.2: Changes in model performance caused by removing predictors from the best model set-up and then refitting the model without the left out variable(s). These results were computed in a leave-one-site-out cross-validation mode. The (-) symbol means that the predictive variable(s) were removed from the RF models. $\mathrm{R}^{2}=$ coefficient of determination; NSE $=$ Nash-Sutcliffe model efficiency coefficient RMSE $=$ root mean squared error; $\mathrm{MAE}=$ mean absolute error; total $\mathrm{n}=716$ for site-years and $\mathrm{n}=126$ for site-average. $\mathrm{NEP}=\mathrm{f}\left(\right.$ age $, \mathrm{g}($ age $\left.), \mathrm{GPP}^{\prime}, \mathrm{T}_{\text {air }}, \mathrm{N}_{\text {deposition }}\right)$.

\begin{tabular}{l|cccc} 
& $\Delta \mathbf{R}^{2}$ & $\Delta \mathbf{~ N S E}$ & $\Delta \mathbf{R M S E}\left(\mathbf{g C ~ m}^{-2} \mathbf{y}^{-1}\right)$ & $\Delta \mathbf{M A E}\left(\mathbf{g C ~ m}^{-2} \mathbf{y}^{-1}\right)$ \\
\hline Site-years & & & & \\
Full model & 0.62 & 0.62 & 180.14 & 131.09 \\
$(-)$ [age $+\mathrm{g}($ age $)]$ & -0.42 & -0.43 & +84.10 & +69.70 \\
$(-) \mathrm{GPP}^{\prime}$ & -0.01 & -0.02 & +4.13 & +1.54 \\
$(-) \mathrm{T}_{\text {air }}$ & -0.01 & -0.01 & +0.52 & -1.06 \\
$(-)\left[\mathrm{GPP}^{\prime}+\mathrm{T}_{\text {air }}\right]$ & -0.05 & -0.06 & +14.54 & +9.81 \\
$(-) \mathrm{N}_{\text {deposition }}$ & -0.01 & -0.01 & +3.40 & +0.38 \\
\hline Site-average & & & & \\
Full model & 0.71 & 0.71 & 162.38 & 117.71 \\
$(-)[$ Age $+\mathrm{g}($ age $)]$ & -0.50 & -0.51 & +98.10 & +85.85 \\
$(-) \mathrm{GPP}^{\prime}$ & -0.04 & -0.04 & +5.98 & +3.10 \\
$(-) \mathrm{T}_{\text {air }}$ & -0.04 & -0.04 & +5.94 & +0.61 \\
$(-)\left[\mathrm{GPP}^{\prime}+\mathrm{T}_{\text {air }}\right]$ & -0.12 & -0.13 & +25.96 & +18.93 \\
$(-) \mathrm{N}_{\text {deposition }}$ & -0.02 & -0.02 & +0.59 & -3.22 \\
\hline
\end{tabular}




\subsection{Discussion}

\subsubsection{Forest age as a key driver of spatiotemporal variability in annual NEP}

Based on theoretical principles of the $\mathrm{C}$ cycle at ecosystem scale, forest age is expected to play a significant role in NEP. Consistent with the early forest dynamics theory on net primary productivity (NPP) trajectories with forest age (Odum, 1969), we empirically found strong support for a non-linear relationship between NEP and forest age, although an age effect was not evident when looking at a univariate relationship, due to spatial variability of other local covariates (Fig. 2.2). Hence, we followed a multi-variate approach (Fig. 2.3) that accounted for the co-variarying effects of other factors that changed in space and time in order to assess the role of age in explaining NEP spatiotemporal variability. Furthermore, RF models have no prior assumption on the functional response between dependent and independent variables, therefore the relevance of forest age (i.e., age $+\mathrm{g}(\mathrm{age})$ ) was also addressed by contrasting the model performance when removing variables (Table 2.2) and by looking at the model residuals across age class (Fig. 2.4). Forest age emerged as the variable that explained most of the spatial and temporal variability in NEP, despite including information on climate and environmental conditions. Photosynthesis and respiration processes drive the link between NEP and forest age, therefore having long term time series of all component fluxes - enabling to establish individual curves per site -, and observationally independent estimates of GPP/ER/NEP, could help disentangling whether the NEP-age dynamics are driven by the links between GPP and age or between ER and age.

Previously, the effect of stand age on the temporal variability of NEP has been demonstrated via the control of age on NPP using a global dataset (Pregitzer and Euskirchen, 2004; Tang et al., 2014). Similarly, forest age plays a dominant role in explaining spatial variability in NEP in the East Asian monsoon region (Gao et al., 2016; Yu et al., 2014). Unlike previous studies, we tested the effect of several drivers on both site-average and site-years, allowing us to evaluate the spatiotemporal NEP variability. Both analyses (i.e. site-average, site-years) showed that forest age was one of the main drivers of NEP variability (Table 2.2). However, some factors were temporally invariant at yearly scales (e.g. soil texture) or did not change over time due to data limitations (e.g. NA), while others could change (e.g. forest age, GPP', $\mathrm{T}_{\text {air }}$ ). Therefore, the lack of temporal variability in these factors could reduce their contribution to the NEP in the site-years analysis.

GPP has been suggested as one of the main drivers of NEP spatial variability (Fernández-Martínez et al., 2014). We found that excluding GPP' from the RF algorithm decreased the model efficiency by 0.02 and 0.04 for site-years and site-average, respectively (Table 2.2). GPP' emerged as superior predictor compared to climate and soil properties (Table 2.A3), likely because it is more closely coupled with NEP, whereas climate and soil properties have variable effects depending on site characteristics. The statistical relation of $\mathrm{GPP}^{\prime}$ to NEP appeared to be significantly stronger 
for stands younger than 20 years, than for intermediate-aged/old-growth forests ( $\geq 2$ 20years) (Fig. 2.A5). In the initial successional pathway, most of the year-to-year variability in NEP is explained by the changes in GPP and climate. However, as forest ecosystems mature and the autotrophic and respiratory processes start to balance each other out, the variations in NEP become more a function of forest age, or time since disturbance, rather than of individual variations in GPP or ER. Having the full representation of stand development stages is important for representing forest spatiotemporal C dynamics after stand-replacing disturbances more realistically (Fig. 2.4). This means that the controls of GPP on NEP are strongly dependent on the distribution of forest age, which emphasizes the relevance of age class distributions for understanding the dynamics of biosphere-atmosphere $\mathrm{CO}_{2}$ exchange. The interactions between forest age and local conditions (e.g. GPP) suggest to move beyond stand age in reflecting changes in plant and soil pools, but also in appropriately parameterizing forest age related changes in ecophysiological mechanisms both at plant and soil levels. Still, we have limited knowledge on the disturbance effects on detrital pools (and thus Rh), the type of transition between previous land cover/use (Carvalhais et al., 2010) followed by different regeneration types (e.g. regrowth, plantation on pasture, former agricultural lands, and afforestation), and site history. These ecosystem conversions may strongly influence ecosystem C balance (Kutsch and Kolari, 2015) and could explain the current bias present for the young forests $(<20$ years) (Fig. 2.4a).

(a)

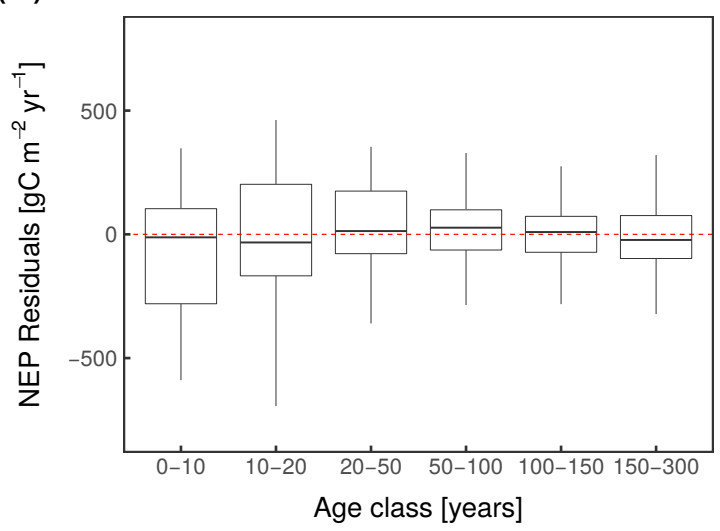

(b)

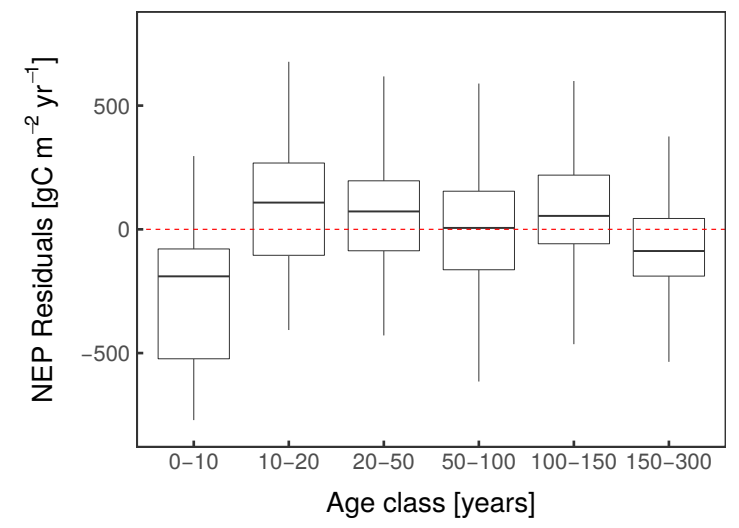

Figure 2.4: Model residuals per age class of (a) a RF model including forest age as a predictive variable, i.e. $\mathbf{N E P}=\mathbf{f}\left(\right.$ age, $\mathbf{g}($ age $\left.), \mathbf{G P P}^{\prime}, \mathrm{T}_{\text {air }}, \mathrm{N}_{\text {deposition }}\right)$ and (b) a RF model excluding forest age as a predictive variable, i.e. $\mathbf{N E P}=\mathbf{f}\left(\mathbf{G P P}^{\prime}, \mathbf{T}_{\text {air }}, \mathbf{N}_{\text {deposition }}\right)$.

\subsubsection{Climate and soil properties controls on spatial annual NEP variability}

While several environmental factors exert controls on NEP, we found that their statistical effect was minor in comparison to forest age. In many cases, sensitivities and even the sign of the relationship between environmental factors and NEP differ among case studies in the literature. For instance, NEP can be positively correlated with $\mathrm{T}_{\text {air }}$ in space (Fernández-Martínez et al., 2014), whereas other studies find only very weak relationships (Law et al., 2002; Piao et al., 
2008; Reichstein et al., 2007b). In boreal regions, air and soil temperature are the main factors affecting interannual NEP variability in old stands, while climatic conditions could not explain temporal patterns of NEP of young stands (Coursolle et al., 2012). Here, $\mathrm{T}_{\text {air }}$ had a modest contribution to explaining NEP variability in the final model (Table 2.2).

Rather than mean temperature, temperature changes in the recent past significantly influence current spatial variability of the forest $C$ sink (Piao et al., 2009). We tested both annual climate anomalies and climate trends (i.e. from 1960 to 2012 based on the CRU dataset) in the final models, but found that they had limited effect on explaining NEP variability (Table 2.A3). Nevertheless, future increases in temperature, changes in precipitation patterns, and more extreme events will likely have significant effects on the $\mathrm{C}$ budgets of forest ecosystems (Thuiller et al., 2011; Trumbore et al., 2015).

Soil characteristics and fertility may play an important role in the spatiotemporal variability of annual NEP (Fernández-Martínez et al., 2014; Janssens et al., 2010; Oren et al., 2001). Ecosystem $\mathrm{C}$ exchanges are generally limited by nutrient availability (often nitrogen) that may increase following disturbance (e.g. stand replacement, harvest). Nitrogen mineralization increases available nitrogen while nitrogen uptake decreases nitrogen availability (Thornton et al., 2002). However, we found that nutrient availability and clay content were not considered statistically strong drivers in explaining NEP variability. This was in contrast to previous studies concluding that nutrient availability is even more important than forest age in explaining across-site forest NEP variability (Fernández-Martínez et al., 2014). This apparent contradiction emerged from the fact that earlier studies used a linear relationship, while we also included a non-linear relationship. We showed that removing a non-linear relationship between NEP and stand age (i.e. g(age)) results in a substantial loss in overall model performance and a significant reduction in the apparent importance of forest age for NEP (Table 2.A4).

Nevertheless, the apparent low contribution of climate, nutrient status, and soil properties to explaining NEP variability can be explained by the fact that their information is already embedded either in forest age or GPP'. The latter is clearly climatically driven, whereas the GPP-NEP dynamic is strongly controlled by forest nutrient availability (Fernández-Martínez et al., 2014). Forest age is likely a superior predictor of spatiotemporal variation in NEP because it integrates relevant ecological information not captured by other single variables. In fact, forest age is rather a composite measure of numerous drivers that are more directly mechanistically coupled with $\mathrm{C}$ cycling processes.

\subsection{Implications}

While GPP, climate, and soil properties are significant factors influencing the variability of NEP across space and time, we conclusively demonstrate that forest age performs as a strong indicator 
of spatiotemporal variability in NEP and is a useful integrated proxy for ecological changes that constrain NEP at the global scale.

Many global ecosystem models rely on simple representations of forest age dynamics and few consider the role of successional changes in C cycling processes (Anderson-Teixeira et al., 2013), which introduces uncertainties into long term simulations of forest $\mathrm{C}$ dynamics (Friedlingstein et al., 2014; Friend et al., 2014). Additionally, given the statistical power of the proposed model in comparison to other state-of-the art approaches (Jung et al., 2011; Tramontana et al., 2016), this study points out new directions towards further developments in bottom-up upscaling exercises based on EC data. Regardless of the modeling strategy, reliable annual maps of forest age distribution and/or disturbance history will be required in order to make accurate predictions of NEP in space and time. Assessing the distribution of forest demography will further support the design of sustainable forest management and climate change mitigation strategies that depend on the effect of forest aging and age class distribution (Pan et al., 2011a; Thuiller et al., 2011; Trumbore et al., 2015).

This study emphasizes the need for increased focus on forest demography, which may amplify or exceed the importance of climate sensitivity for predicting the future of the terrestrial $\mathrm{C}$ cycle. 


\section{A Appendix}

This appendix represents the supplementary materials of the presented publication.

Sites used in this study 
Table 2.A1: Sites used in this study. Lat $=$ latitude, Long $=$ longitude, $\mathrm{NEP}=$ net ecosystem production, $\mathrm{GPP}=$ gross primary production, $\mathrm{T}_{\text {air }}=$ mean annual temperature, $\mathrm{P}=$ total annual precipitation, $\mathrm{NA}=$ nutrient availability, Dist $=$ disturbance events used to characterize the stand age of each site where the meaning of the numbers 1-4 are defined in section 2.A.2.

\begin{tabular}{|c|c|c|c|c|c|c|c|c|c|}
\hline Site & $\begin{array}{l}\text { Lat } \\
{\left[{ }^{\circ} \mathrm{N}\right]}\end{array}$ & $\begin{array}{l}\text { Long } \\
{\left[{ }^{\circ} \mathrm{E}\right]}\end{array}$ & $\begin{array}{l}\text { NEP } \\
{\left[\mathrm{gC} \mathrm{m}^{-2} \mathrm{y}^{-1}\right]}\end{array}$ & $\begin{array}{l}\text { GPP } \\
{\left[\mathrm{gC} \mathrm{m}^{-2} \mathrm{y}^{-1}\right]}\end{array}$ & $\begin{array}{l}\text { Age } \\
\text { [years] }\end{array}$ & $\begin{array}{l}\mathrm{T}_{\text {air }} \\
{\left[{ }^{\circ} \mathrm{C}\right]}\end{array}$ & $\begin{array}{l}\mathrm{P} \\
{\left[\mathrm{mm} \mathrm{y}^{-1}\right]}\end{array}$ & NA & Dist \\
\hline AU-Cum & -33.613 & 150.722 & 172.67 & 1198.28 & 300.00 & 18.40 & 595.85 & M & 1 \\
\hline AU-How & -12.494 & 131.152 & 435.47 & 2070.71 & 110.00 & 26.99 & 1681.15 & M & 1 \\
\hline AU-Rob & -17.117 & 145.630 & 657.52 & 2116.39 & 198.00 & 21.72 & 2193.83 & M & 1 \\
\hline AU-Tum & -35.656 & 148.151 & 633.15 & 3328.78 & 83.00 & 9.78 & 889.26 & M & 1 \\
\hline AU-Wom & -37.422 & 144.094 & 681.83 & 1737.04 & 32.00 & 11.97 & 786.50 & M & 1 \\
\hline BE-Bra & 51.309 & 4.520 & 118.73 & 1426.98 & 78.00 & 10.82 & 824.67 & $\mathrm{~L}$ & 1 \\
\hline BE-Vie & 50.305 & 5.998 & 454.14 & 1755.48 & 94.00 & 8.34 & 966.29 & M & 1 \\
\hline BR-Sa1 & -2.856 & -54.958 & -26.53 & 3342.92 & 300.00 & 25.96 & 2596.91 & $\mathrm{~L}$ & 1 \\
\hline $\mathrm{BR}-\mathrm{Sa} 3$ & -3.018 & -54.971 & 105.85 & 3045.54 & 2 & 300.00 & 1408.04 & $\mathrm{~L}$ & 1 \\
\hline CA-Ca1 & 49.867 & -125.333 & 391.49 & 2232.29 & 60.00 & 8.39 & 1298.95 & $\mathrm{~L}$ & 1 \\
\hline $\mathrm{CA}-\mathrm{Ca} 2$ & 49.870 & -125.291 & -518.73 & 588.30 & 2.00 & 8.89 & 1214.01 & $\mathrm{~L}$ & 1 \\
\hline $\mathrm{CA}-\mathrm{Ca} 3$ & 49.534 & -124.900 & 11.55 & 1342.57 & 16.00 & 9.66 & 1543.54 & $\mathrm{~L}$ & 1 \\
\hline CA-Gro & 48.216 & -82.155 & 117.79 & 1065.12 & 78.00 & 3.60 & 770.32 & M & 1 \\
\hline CA-Man & 55.879 & -98.480 & 22.56 & 687.54 & 161.00 & -1.11 & 310.38 & $\mathrm{~L}$ & 1 \\
\hline CA-NS1 & 55.879 & -98.483 & 89.03 & 739.28 & 154.00 & 0.22 & 215.28 & $\mathrm{~L}$ & 1 \\
\hline CA-NS2 & 55.905 & -98.524 & 172.45 & 948.37 & 73.00 & -3.70 & 275.29 & $\mathrm{~L}$ & 1 \\
\hline CA-NS3 & 55.911 & -98.382 & 60.76 & 570.64 & 39.00 & -2.93 & 196.49 & $\mathrm{~L}$ & 1 \\
\hline CA-NS5 & 55.863 & -98.485 & 80.68 & 695.77 & 23.00 & -3.42 & 220.66 & $\mathrm{~L}$ & 1 \\
\hline CA-NS6 & 55.916 & -98.964 & 15.21 & 421.48 & 14.00 & -1.11 & 201.88 & $\mathrm{~L}$ & 1 \\
\hline CA-NS7 & 56.635 & -99.948 & -103.98 & 422.34 & 6.00 & -2.09 & 235.75 & $\mathrm{~L}$ & 1 \\
\hline CA-Oas & 53.628 & -106.197 & 122.27 & 1113.43 & 80.00 & 1.96 & 479.07 & $\mathrm{~L}$ & 1 \\
\hline CA-Obs & 53.987 & -105.117 & 38.26 & 841.18 & 112.00 & 1.12 & 495.74 & $\mathrm{~L}$ & 1 \\
\hline CA-Ojp & 53.916 & -104.692 & 46.83 & 572.34 & 88.00 & 1.40 & 454.21 & $\mathrm{~L}$ & 1 \\
\hline CA-Qcu & 49.267 & -74.036 & -146.72 & 321.05 & 4.00 & 1.16 & 930.16 & $\mathrm{~L}$ & 1 \\
\hline CA-Qfo & 49.692 & -74.342 & 3.47 & 648.33 & 102.00 & 1.13 & 939.19 & $\mathrm{~L}$ & 1 \\
\hline CA-SJ1 & 53.908 & -104.655 & -25.48 & 373.52 & 10.00 & 0.57 & 734.31 & $\mathrm{~L}$ & 1 \\
\hline CA-SJ2 & 53.944 & -104.649 & -130.15 & 89.81 & 2.00 & -0.03 & 674.45 & $\mathrm{~L}$ & 1 \\
\hline
\end{tabular}

Continued on next page 
Table 2.A1 - Continued from previous page

\begin{tabular}{|c|c|c|c|c|c|c|c|c|c|}
\hline Site & $\begin{array}{l}\text { Lat } \\
{\left[{ }^{\circ} \mathrm{N}\right]} \\
\end{array}$ & $\begin{array}{l}\text { Long } \\
{\left[{ }^{\circ} \mathrm{E}\right]} \\
\end{array}$ & $\begin{array}{l}\text { NEP } \\
{\left[\mathrm{gC} \mathrm{m}^{-2} \mathrm{y}^{-1}\right]}\end{array}$ & $\begin{array}{l}\text { GPP } \\
{\left[\mathrm{gC} \mathrm{m}^{-2} \mathrm{y}^{-1}\right]}\end{array}$ & $\begin{array}{l}\text { Age } \\
\text { [years] }\end{array}$ & $\begin{array}{l}\mathrm{T}_{a i r} \\
{\left[{ }^{\circ} \mathrm{C}\right]}\end{array}$ & $\begin{array}{l}\mathrm{P} \\
{\left[\mathrm{mm} \mathrm{y}^{-1}\right]}\end{array}$ & NA & Dist \\
\hline CA-SJ3 & 53.875 & -104.649 & 90.34 & 535.52 & 30.00 & 1.80 & 1137.16 & $\mathrm{~L}$ & 1 \\
\hline CA-TP1 & 42.660 & -80.559 & 183.45 & 1301.86 & 9.00 & 9.03 & 1090.20 & M & 1 \\
\hline CA-TP3 & 42.706 & -80.348 & 451.11 & 1682.59 & 37.00 & 8.91 & 1145.72 & M & 1 \\
\hline CA-TP4 & 42.710 & -80.357 & 90.00 & 1508.94 & 70.00 & 8.52 & 980.58 & M & 1 \\
\hline CA-TPD & 42.635 & -80.557 & 299.88 & 1499.02 & 98.00 & 9.66 & 920.81 & M & 1 \\
\hline CA-WP1 & 54.953 & -112.467 & 250.03 & 693.32 & 136.00 & 1.96 & 439.89 & $\mathrm{~L}$ & 1 \\
\hline CH-Dav & 46.815 & 9.855 & 116.39 & 1238.17 & 222.00 & 3.54 & 849.99 & $\mathrm{~L}$ & 1 \\
\hline CH-Lae & 47.478 & 8.365 & 662.11 & 1931.91 & 184.00 & 7.72 & 1195.59 & M & 1 \\
\hline CN-Cha & 42.402 & 128.095 & 268.85 & 1496.07 & 300.00 & 4.35 & 466.14 & $\mathrm{H}$ & 1 \\
\hline CN-Din & 23.173 & 112.536 & 469.94 & 1462.56 & 96.00 & 20.40 & 1411.43 & $\mathrm{~L}$ & 1 \\
\hline CN-Qia & 26.741 & 115.058 & 573.53 & 1812.07 & 19.00 & 18.24 & 1169.89 & $\mathrm{~L}$ & 1 \\
\hline CZ-BK1 & 49.502 & 18.536 & 831.75 & 1844.97 & 31.00 & 6.53 & 1102.62 & M & 1 \\
\hline DE-Bay & 50.141 & 11.866 & -45.49 & 1354.17 & 54.00 & 6.30 & 1241.17 & $\mathrm{~L}$ & 1 \\
\hline DE-Hai & 51.079 & 10.453 & 564.43 & 1662.84 & 254.00 & 8.47 & 756.57 & $\mathrm{H}$ & 1 \\
\hline DE-Har & 47.934 & 7.6009 & 618.93 & 1530.12 & 42.00 & 11.12 & 619.00 & M & 1 \\
\hline DE-Lkb & 49.099 & 13.304 & -267.09 & 510.12 & 2.00 & 5.48 & 873.63 & M & 4 \\
\hline DE-Lnf & 51.328 & 10.367 & 594.26 & 1685.98 & 117.00 & 8.07 & 541.38 & M & 1 \\
\hline DE-Meh & 51.275 & 10.655 & -3.25 & 1136.89 & 2.00 & 8.80 & 520.43 & $\mathrm{H}$ & 1 \\
\hline DE-Obe & 50.783 & 13.719 & 372.53 & 1805.06 & 76.00 & 6.49 & 1044.06 & M & 1 \\
\hline DE-Tha & 50.963 & 13.566 & 613.99 & 1980.87 & 118.00 & 8.91 & 841.43 & M & 1 \\
\hline DE-Wet & 50.453 & 11.457 & 82.15 & 1529.44 & 54.00 & 6.41 & 1021.64 & $\mathrm{~L}$ & 1 \\
\hline DK-Sor & 55.485 & 11.644 & 167.33 & 1952.19 & 85.00 & 8.40 & 854.38 & $\mathrm{H}$ & 1 \\
\hline ES-ES1 & 39.346 & -0.318 & 404.01 & 1432.53 & 116.00 & 17.41 & 607.96 & $\mathrm{~L}$ & 1 \\
\hline ES-LMa & 39.941 & -5.773 & 122.29 & 1069.11 & 148.00 & 16.16 & 778.24 & $\mathrm{~L}$ & 1 \\
\hline FI-Hyy & 61.847 & 24.29 & 219.40 & 1118.01 & 46.00 & 4.40 & 607.38 & $\mathrm{~L}$ & 1 \\
\hline FI-Sod & 67.361 & 26.637 & -101.60 & 598.89 & 83.00 & 0.77 & 534.58 & $\mathrm{~L}$ & 1 \\
\hline FR-Fon & 48.476 & 2.780 & 651.50 & 1835.12 & 161.00 & 11.25 & 669.27 & $\mathrm{H}$ & 1 \\
\hline FR-Hes & 48.674 & 7.0646 & 333.77 & 1599.72 & 37.00 & 10.12 & 957.32 & $\mathrm{~L}$ & 1 \\
\hline FR-LBr & 44.720 & -0.770 & 299.94 & 1726.74 & 34.00 & 13.40 & 939.40 & $\mathrm{~L}$ & 1 \\
\hline FR-Pue & 43.741 & 3.595 & 233.42 & 1214.59 & 64.00 & 13.74 & 937.66 & M & 1 \\
\hline
\end{tabular}


Table 2.A1 - Continued from previous page

\begin{tabular}{|c|c|c|c|c|c|c|c|c|c|}
\hline Site & $\begin{array}{l}\text { Lat } \\
{\left[{ }^{\circ} \mathrm{N}\right]}\end{array}$ & $\begin{array}{l}\text { Long } \\
{\left[{ }^{\circ} \mathrm{E}\right]}\end{array}$ & $\begin{array}{l}\text { NEP } \\
{\left[\mathrm{gC} \mathrm{m}^{-2} \mathrm{y}^{-1}\right]}\end{array}$ & $\begin{array}{l}\text { GPP } \\
{\left[\mathrm{gC} \mathrm{m}^{-2} \mathrm{y}^{-1}\right]}\end{array}$ & $\begin{array}{l}\text { Age } \\
\text { [years] }\end{array}$ & $\begin{array}{l}\mathrm{T}_{\text {air }} \\
{\left[{ }^{\circ} \mathrm{C}\right]}\end{array}$ & $\begin{array}{l}\mathrm{P} \\
{\left[\mathrm{mm} \mathrm{y}^{-1}\right]}\end{array}$ & NA & Dist \\
\hline GF-Guy & 5.278 & -52.924 & 154.65 & 3724.44 & 300.00 & 25.61 & 3110.49 & $\mathrm{~L}$ & 1 \\
\hline IL-Yat & 31.344 & 35.051 & 201.20 & 649.62 & 39.00 & 18.01 & 265.50 & M & 1 \\
\hline IT-Col & 41.849 & 13.588 & 537.18 & 1300.52 & 180.00 & 7.28 & 999.41 & $\mathrm{H}$ & 1 \\
\hline IT-Cp2 & 41.704 & 12.357 & 590.85 & 2031.64 & 63.00 & 15.82 & 915.08 & M & 1 \\
\hline IT-Cpz & 41.705 & 12.376 & 488.78 & 1936.53 & 56.00 & 16.19 & 742.46 & $\mathrm{~L}$ & 1 \\
\hline IT-La2 & 45.9542 & 11.2853 & 1049.37 & 2108.42 & 89.00 & 6.76 & 1177.20 & $\mathrm{H}$ & 1 \\
\hline IT-LMa & 45.581 & 7.154 & 595.15 & 909.33 & 71.00 & 14.10 & 804.15 & $\mathrm{H}$ & 1 \\
\hline IT-Noe & 40.606 & 8.151 & 205.46 & 1122.14 & 49.00 & 16.31 & 586.35 & M & 1 \\
\hline IT-Non & 44.689 & 11.088 & 481.36 & 1346.20 & 10.00 & 13.59 & 1221.79 & $\mathrm{H}$ & 1 \\
\hline IT-PT1 & 45.200 & 9.061 & 494.79 & 1477.01 & 13.00 & 14.27 & 540.29 & $\mathrm{~L}$ & 1 \\
\hline IT-Ren & 46.587 & 11.434 & 693.16 & 1498.57 & 188.00 & 4.57 & 904.97 & M & 1 \\
\hline IT-Ro1 & 42.408 & 11.930 & 229.25 & 1550.23 & 10.00 & 15.47 & 851.96 & $\mathrm{H}$ & 4 \\
\hline IT-Ro2 & 42.390 & 11.920 & 686.27 & 1443.09 & 19.00 & 15.36 & 802.84 & $\mathrm{H}$ & 4 \\
\hline IT-SR2 & 43.732 & 10.291 & 351.58 & 2343.56 & 64.00 & 15.47 & 1328.66 & $\mathrm{~L}$ & 1 \\
\hline IT-SRo & 43.727 & 10.284 & 369.82 & 1931.90 & 54.00 & 15.38 & 820.96 & $\mathrm{~L}$ & 1 \\
\hline JP-Tak & 36.146 & 137.423 & 308.20 & 1087.49 & 72.00 & 6.60 & 2.26 & $\mathrm{H}$ & 1 \\
\hline JP-Tef & 45.056 & 142.106 & -307.58 & 979.21 & 84.00 & 5.88 & 965.85 & $\mathrm{~L}$ & 4 \\
\hline JP-Tom & 42.739 & 141.514 & 331.19 & 1710.27 & 48.00 & 6.28 & 1065.99 & $\mathrm{~L}$ & 1 \\
\hline MY-PSO & 2.973 & 102.306 & 129.89 & 2554.45 & 106.00 & 25.34 & 1864.85 & $\mathrm{~L}$ & 1 \\
\hline NL-Loo & 52.166 & 5.743 & 450.75 & 1637.40 & 106.00 & 10.08 & 803.22 & M & 1 \\
\hline PA-SPn & 9.318 & -79.634 & 441.80 & 2088.30 & 7.00 & 25.16 & 2073.59 & M & 1 \\
\hline PT-Esp & 38.639 & -8.601 & 629.21 & 1842.15 & 12.00 & 16.36 & 592.53 & $\mathrm{~L}$ & 1 \\
\hline PT-Mi1 & 38.540 & -8.000 & -39.88 & 828.81 & 88.00 & 15.80 & 243.84 & $\mathrm{~L}$ & 1 \\
\hline RU-Fyo & 56.461 & 32.922 & -137.79 & 1496.93 & 236.00 & 5.23 & 560.63 & $\mathrm{~L}$ & 1 \\
\hline SE-Fla & 64.112 & 19.456 & 59.17 & 704.12 & 37.00 & 3.06 & 703.79 & $\mathrm{~L}$ & 1 \\
\hline SE-Nor & 60.086 & 17.479 & -75.49 & 1155.43 & 105.00 & 6.06 & 703.73 & $\mathrm{~L}$ & 1 \\
\hline SE-Sk1 & 60.125 & 17.918 & -186.43 & 461.16 & 2.00 & 9.30 & 0.59 & $\mathrm{H}$ & 1 \\
\hline SE-Sk2 & 60.129 & 17.840 & 66.34 & 674.11 & 33.00 & 6.69 & 0.58 & $\mathrm{H}$ & 1 \\
\hline UK-Gri & 56.607 & -3.798 & 515.53 & 1710.60 & 21.00 & 7.48 & 1115.84 & $\mathrm{H}$ & 1 \\
\hline UK-Ham & 51.120 & -0.860 & 595.09 & 2111.61 & 64.00 & 10.47 & 796.70 & $\mathrm{H}$ & 1 \\
\hline
\end{tabular}


Table 2.A1 - Continued from previous page

\begin{tabular}{|c|c|c|c|c|c|c|c|c|c|}
\hline Site & $\begin{array}{l}\text { Lat } \\
{\left[{ }^{\circ} \mathrm{N}\right]}\end{array}$ & $\begin{array}{l}\text { Long } \\
{\left[{ }^{\circ} \mathrm{E}\right]}\end{array}$ & $\begin{array}{l}\text { NEP } \\
{\left[\mathrm{gC} \mathrm{m}^{-2} \mathrm{y}^{-1}\right]}\end{array}$ & $\begin{array}{l}\text { GPP } \\
{\left[\mathrm{gC} \mathrm{m}^{-2} \mathrm{y}^{-1}\right]}\end{array}$ & $\begin{array}{l}\text { Age } \\
\text { [years] }\end{array}$ & $\begin{array}{l}\mathrm{T}_{\text {air }} \\
{\left[{ }^{\circ} \mathrm{C}\right]}\end{array}$ & $\begin{array}{l}\mathrm{P} \\
{\left[\mathrm{mm} \mathrm{y}^{-1}\right]}\end{array}$ & NA & Dist \\
\hline US-Bar & 44.064 & -71.288 & 367.15 & 1102.15 & 128.00 & 7.15 & 1254.52 & $\mathrm{M}$ & 1 \\
\hline US-Blo & 38.895 & -120.632 & 176.75 & 1965.20 & 13.00 & 10.97 & 1526.95 & $\mathrm{~L}$ & 1 \\
\hline US-Bn1 & 63.919 & -145.378 & 268.68 & 524.40 & 83.00 & -1.10 & 249.15 & $\mathrm{~L}$ & 1 \\
\hline US-Bn2 & 63.919 & -145.378 & 239.81 & 512.99 & 16.00 & -0.48 & 249.15 & $\mathrm{~L}$ & 1 \\
\hline US-Bn3 & 63.922 & -145.744 & 91.85 & 238.46 & 4.00 & -0.71 & 249.15 & $\mathrm{~L}$ & 1 \\
\hline US-Dk3 & 35.978 & -79.094 & 756.04 & 2245.52 & 21.00 & 14.95 & 1037.37 & $\mathrm{~L}$ & 1 \\
\hline US-GBT & 41.365 & -106.239 & 528.98 & 718.88 & 176.00 & -0.03 & 424.41 & M & 1 \\
\hline US-GLE & 41.366 & -106.239 & -83.52 & 618.81 & 184.00 & -0.03 & 1405.35 & M & 1 \\
\hline US-Ha1 & 42.537 & -72.171 & 199.79 & 1506.46 & 96.00 & 8.32 & 1139.66 & $\mathrm{~L}$ & 1 \\
\hline US-Hol & 45.204 & -68.740 & 255.50 & 1468.32 & 206.00 & 6.60 & 815.29 & $\mathrm{~L}$ & 1 \\
\hline US-Ho2 & 45.209 & -68.747 & 231.09 & 1342.73 & 208.00 & 6.51 & 787.37 & $\mathrm{~L}$ & 1 \\
\hline US-Los & 46.082 & -89.979 & 48.07 & 783.45 & 35.00 & 4.92 & 729.34 & $\mathrm{~L}$ & 1 \\
\hline US-LPH & 42.541 & -72.184 & 368.35 & 1233.06 & 98.00 & 7.33 & 2520.50 & M & 1 \\
\hline US-Me2 & 44.452 & -121.557 & 604.98 & 1616.96 & 94.00 & 7.31 & 457.49 & M & 1 \\
\hline US-Me3 & 44.315 & -121.607 & 48.02 & 781.76 & 20.00 & 8.20 & 367.11 & M & 1 \\
\hline US-Me5 & 44.437 & -121.566 & 137.85 & 818.46 & 24.00 & 7.89 & 412.90 & M & 1 \\
\hline US-Me6 & 44.323 & -121.607 & 180.66 & 882.75 & 22.00 & 7.71 & 409.02 & M & 1 \\
\hline US-MMS & 39.323 & -86.413 & 423.08 & 1718.92 & 95.00 & 12.41 & 1084.11 & M & 1 \\
\hline US-MOz & 38.744 & -92.199 & 353.74 & 1424.79 & 78.00 & 13.52 & 873.63 & $\mathrm{~L}$ & 1 \\
\hline US-NC1 & 35.811 & -76.711 & -178.28 & 1629.06 & 2.00 & 15.59 & 1056.09 & M & 1 \\
\hline US-NC2 & 35.803 & -76.667 & 936.24 & 2724.41 & 14.00 & 15.92 & 1272.59 & M & 1 \\
\hline US-NR1 & 40.032 & -105.546 & 168.94 & 846.05 & 110.00 & 2.28 & 726.36 & $\mathrm{~L}$ & 1 \\
\hline US-Oho & 41.554 & -83.843 & 827.98 & 1776.81 & 50.00 & 10.52 & 818.23 & $\mathrm{~L}$ & 1 \\
\hline US-PFa & 45.945 & -90.272 & 9.37 & 959.85 & 150.00 & 5.72 & 600.94 & $\mathrm{H}$ & 1 \\
\hline US-Prr & 65.123 & -147.487 & 123.43 & 496.91 & 98.00 & -2.02 & 382.16 & $\mathrm{~L}$ & 1 \\
\hline US-SO2 & 33.373 & -116.622 & 59.07 & 283.07 & 2.00 & 14.43 & 532.54 & M & 4 \\
\hline US-SO3 & 33.377 & -116.622 & 86.86 & 341.03 & 2.00 & 14.79 & 454.00 & M & 4 \\
\hline US-SP1 & 29.738 & -82.218 & 161.84 & 1722.32 & 63.00 & 19.73 & 53.86 & $\mathrm{~L}$ & 1 \\
\hline US-SP2 & 29.764 & -82.244 & 162.00 & 2178.24 & 4.00 & 19.70 & 1118.41 & $\mathrm{~L}$ & 1 \\
\hline US-SP3 & 29.754 & -82.163 & 542.14 & 1875.13 & 12.00 & 20.14 & 1075.60 & $\mathrm{~L}$ & 1 \\
\hline
\end{tabular}


Table 2.A1 - Continued from previous page

\begin{tabular}{clllllllll}
\hline Site & $\begin{array}{l}\text { Lat } \\
{\left[{ }^{\circ} \mathrm{N}\right]}\end{array}$ & $\begin{array}{l}\text { Long } \\
{\left[{ }^{\circ} \mathrm{E}\right]}\end{array}$ & $\begin{array}{l}\mathrm{NEP} \\
{\left[\mathrm{gC} \mathrm{m}^{-2} \mathrm{y}^{-1}\right]}\end{array}$ & $\begin{array}{l}\mathrm{GPP} \\
{\left[\mathrm{gC} \mathrm{m}^{-2} \mathrm{y}^{-1}\right]}\end{array}$ & $\begin{array}{l}\text { Age } \\
{[\text { years }]}\end{array}$ & $\begin{array}{l}\mathrm{T}_{\text {air }} \\
{\left[{ }^{\circ} \mathrm{C}\right]}\end{array}$ & $\begin{array}{l}\mathrm{P} \\
{\left[\mathrm{mm} \mathrm{y}^{-1}\right]}\end{array}$ & NA & Dist \\
\hline US-SRM & 31.821 & -110.866 & -24.81 & 283.18 & 201.00 & 19.01 & 333.19 & $\mathrm{~L}$ & 1 \\
US-Syv & 46.242 & -89.347 & 80.38 & 1171.41 & 300.00 & 3.92 & 692.48 & $\mathrm{~L}$ & 1 \\
US-UMB & 45.559 & -84.713 & 268.69 & 1299.05 & 93.00 & 7.15 & 613.06 & $\mathrm{~L}$ & 1 \\
US-UMd & 45.562 & -84.697 & 316.98 & 1332.29 & 90.00 & 7.36 & 727.64 & $\mathrm{~L}$ & 1 \\
US-WBW & 35.958 & -84.287 & 815.94 & 1453.80 & 110.00 & 14.64 & 1.52 & $\mathrm{~L}$ & 1 \\
US-WCr & 45.805 & -90.079 & 282.37 & 1173.52 & 96.00 & 5.77 & 695.24 & $\mathrm{M}$ & 1 \\
US-Wrc & 45.820 & -121.952 & 347.08 & 1625.95 & 300.00 & 9.41 & 2139.87 & $\mathrm{M}$ & 1 \\
VU-Coc & -15.443 & 167.192 & 358.24 & 3499.22 & 20.00 & 24.71 & 2734.43 & $\mathrm{H}$ & 1 \\
ZM-Mon & -15.434 & 23.253 & -147.63 & 1566.22 & 88.00 & 22.20 & 682.80 & $\mathrm{M}$ & 4 \\
\hline
\end{tabular}




\section{Characterization of forest age estimates}

In order to define forest age, we looked at four different scenarii that were reported in the biological, ancillary, disturbance and metadata database (BADM), in publications, or provided by the PIs:

1. Complete stand replacement events: reports of a clear cut, a crop to tree cover (afforestation) transition, or a stand replacing fire event (either natural or a controlled experiment). In these cases the event was reported as to have had covered the whole area under the observational footprint of the EC tower. We used this information to set the age of the site.

2. Minor stand disturbance events: for some sites, the BADM reports insect outbreaks or wind throws covering minor parts of the stand and footprint of the EC towers. This information was rarely qualitative, but most of the time refered to low/minor intensity disturbance. In these cases, we did not adjust the age of the stand.

3. Marginal logging events: the BADM reports around 25 sites in which selective logging event harvesting occurred. In four cases, there were some quantitative information on the amount of biomass removed (e.g. BR-Sa3: selectively logged in September 2001, where only $5 \%$ of the aboveground biomass were removed; US-SP1: $27 \%$ of basal area removed). In fact, most of the time, only qualitative information on the intensity of these disturbance (i.e. minor or low) was provided or the PIs only reported that a disturbance event occurred but with no information on its intensity. Therefore, it was not possible to attribute an age change as there was no information on tree density, or age of the trees logged. Like for the minor stand disturbance events, we opted for not adjusting the age of the stand. Other qualitative disturbance intensity reports fell under case \#4

4. Partial substantial disturbance: there were cases where the sites (i.e. 5 sites) underwent intense disturbance events (i.e. fire) impacting a majority of the trees, which were reported in the BADM, or publications. In all these cases, we considered that the age was the difference between the flux observation year and the year of disturbance. When the disturbance occurred prior to the observational period there was no other record to resource than the site reports of a large and intense disturbance. Perhaps these were the cases where the highest uncertainties in age emerged that could contribute to further improvements in the models.

\section{Eddy-covariance processing}

We used the night time partitioning, variable ustar, and reference flux observation versions. Data processing included: storage-correction; spike and $\mathrm{u}^{*}$-filtering; flux partitioning (Reichstein et al., 2005). Only sites with more than $80 \%$ of the original or good quality gap-filled data were included in this analysis. 


\section{Latent heat flux filtering and GPP' computation}

We filtered out daily LE estimates below the $10 \%$ quantile within site and where air temperature was lower than $10^{\circ} \mathrm{C}$ and computed $\mathrm{GPP}^{\prime}$ as the annual sum of the ratio between LE and the square root of VPD computed at daily day-time scale.

\section{Spuriousness analysis}

We computed the coefficient of spurious correlation (Rsc) adapted from :

- $\mathrm{GPP}=\mathrm{NEE}+\mathrm{ER}$

- $\mathrm{NEE}=-\mathrm{GPP}+\mathrm{ER}$

- $\mathrm{ER}=\mathrm{NEE}+\mathrm{GPP}$

The Rsc coefficient can then be calculated as follows:

- $\mathrm{Rsc}=-\operatorname{var}(\mathrm{ER}) /\left[(\operatorname{var}(\mathrm{GPP})+\operatorname{var}(\mathrm{ER}))^{0.5} \mathrm{x}(\operatorname{var}(\mathrm{NEE})+\operatorname{var}(\mathrm{ER}))^{0.5}\right]$

We found that Rsc is equal to -0.58 to -0.57 for site-years and site-average, respectively. The Rsc estimates suggested that there was a moderate spurious correlation between net ecosystem exchange (NEE) and GPP at annual scale, although we showed that Rsc varied from low to moderate amongst sites with a mean value of -0.48 . This was suggesting that the correlation between GPP and NEP at annual scales was unlikely to be an artifact from the partitioning.

\section{Comparision between the different forest age models}

Table 2.A2: Model performance comparision between Amiro et al. (2010), Coursolle et al. (2012), and Tang et al. (2014) models. NSE estimates are reported in the table.

\begin{tabular}{l|ll} 
& Site-years & Site-average \\
\hline Amiro & 0.62 & 0.71 \\
Coursolle & 0.38 & 0.41 \\
Tang & 0.40 & 0.44
\end{tabular}




\section{Variable selection procedure}

Table 2.A3: Results of the variable selection procedure. Only the five best variables were kept in the final Random Forest model. The Z-scores were computed by dividing the average accuracy loss when permuting variables by its standard deviation.

\begin{tabular}{|c|c|c|c|c|}
\hline & mean Z-score & median Z-score & min Z-score & $\max$ Z-score \\
\hline g(age) & 33.06 & 33.40 & 30.39 & 34.80 \\
\hline Forest age & 24.43 & 24.43 & 22.46 & 27.14 \\
\hline $\mathrm{GPP}^{\prime}$ & 23.23 & 23.31 & 20.47 & 24.93 \\
\hline $\mathrm{N}_{\text {deposition }}$ & 21.35 & 21.33 & 19.99 & 22.66 \\
\hline $\mathrm{T}_{\text {air }}$ & 20.47 & 20.60 & 18.19 & 21.92 \\
\hline Silt content & 18.99 & 18.98 & 17.34 & 20.61 \\
\hline $\mathrm{T}_{\text {air }}$ trend & 18.02 & 18.13 & 14.37 & 20.20 \\
\hline P trend & 17.18 & 17.22 & 14.18 & 18.72 \\
\hline Sand content & 16.05 & 16.16 & 14.04 & 17.40 \\
\hline Clay content & 15.82 & 15.94 & 14.31 & 17.45 \\
\hline Global radiation & 15.39 & 15.21 & 14.23 & 18.48 \\
\hline $\mathrm{P}$ & 12.4012 .27 & 10.62 & 14.32 & \\
\hline Nutrient availability & 11.80 & 11.70 & 10.52 & 13.96 \\
\hline Forest management & 8.19 & 8.20 & 7.11 & 9.00 \\
\hline P anomaly & 3.68 & 3.67 & 1.00 & 5.97 \\
\hline $\mathrm{T}_{\text {air }}$ anomaly & 1.40 & 1.38 & -0.80 & 3.15 \\
\hline
\end{tabular}

\section{Changes in model performance by subtracting g(age) from model formluation}

Table 2.A4: Changes in model performance by subtracting forest age (not using $g($ age)) from model formluation. $\mathrm{NEP}=\mathrm{f}\left(\right.$ age $\left., \mathrm{GPP}^{\prime}, \mathrm{T}_{\text {air }}, \mathrm{N}_{\text {deposition }}\right) . \mathrm{R}^{2}=$ coefficient of determination; $\mathrm{NSE}=$ modelling efficiency; RMSE $=$ root mean square error; $\mathrm{MAE}=$ mean absolute error; total $n=716$ for site-years and $n=126$ for site-average.

\begin{tabular}{l|cccc} 
& $\Delta \mathbf{R}^{2}$ & $\Delta \mathbf{N S E}$ & $\Delta \mathbf{R M S E}\left(\mathbf{g C ~ m}^{-2} \mathbf{y}^{-1}\right)$ & $\Delta \mathbf{M A E}\left(\mathbf{g C ~ m}^{-2} \mathbf{y}^{-1}\right)$ \\
\hline Site-years & & & & \\
Full model & 0.29 & 0.29 & 248.23 & 189.05 \\
(-) Age & -0.08 & -0.10 & +16.01 & +11.75 \\
\hline Site-average & & & & 180.85 \\
Full model & 0.31 & 0.31 & 242.37 & +22.71 \\
(-) Age & -0.10 & -0.11 & +18.11 & \\
\hline
\end{tabular}




\section{Geographical distribution of the sites used in the analysis}

(a)

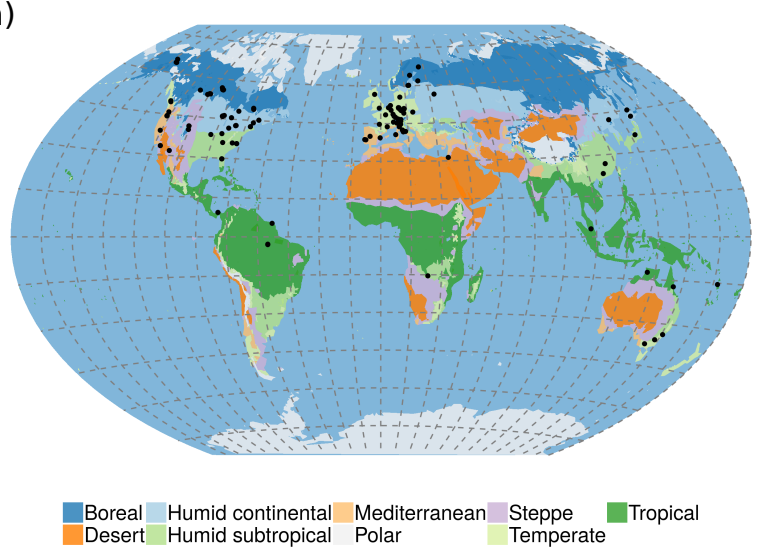

(b)

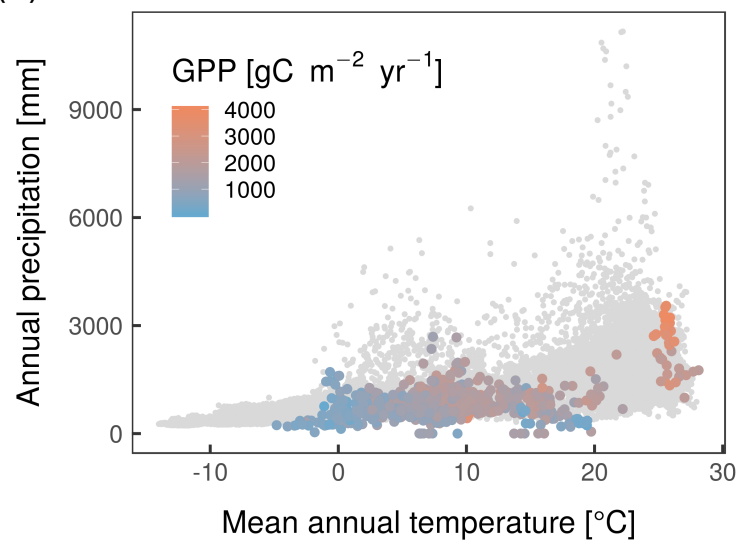

Figure 2.A1: Geographical (a) and climatological (b) distribution of the sites used in the analysis. In (a) the black dots indicate the location of the different FLUXNET sites. In (b) the color code in the legend represents annual GPP $\left[\mathrm{gC} \mathrm{m}^{-2} \mathrm{y}^{-1}\right]$. The data points colored according to the color bar represent the sites precipitation-temperature combinations (climate-space) of the measurements at annual scale while the gray dots represent the climate space of the global forest ecosystems. The climate classification system was derived from Köppen Geiger A2 Scenario 2001-2025. 
Forest age distribution across climate zones and plant functional types

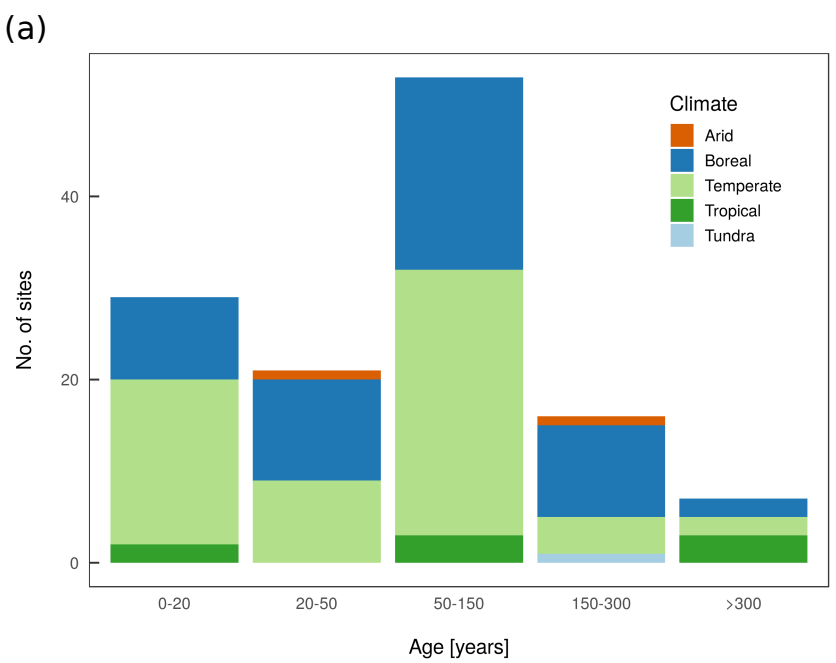

(b)

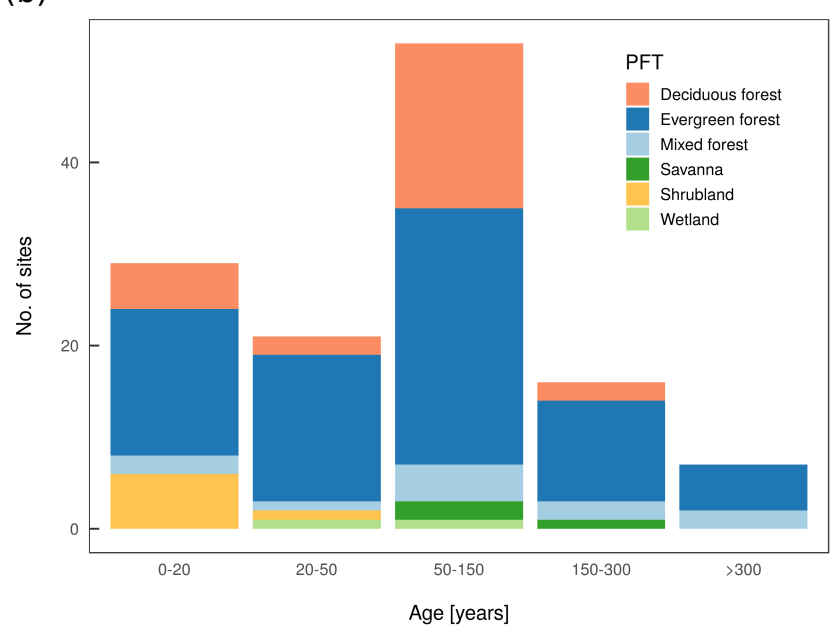

Figure 2.A2: Histogram of forest age from young to old-growth forests in our dataset by (a) major climate zones and (b) plant functional types. 
Correlation between a series of variables

(a)

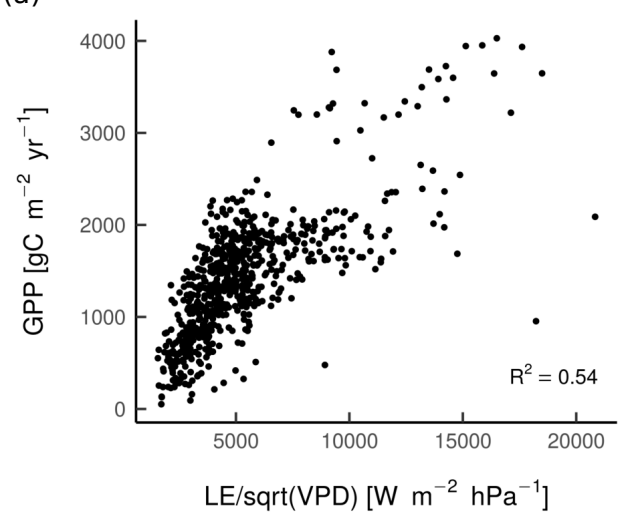

(c)

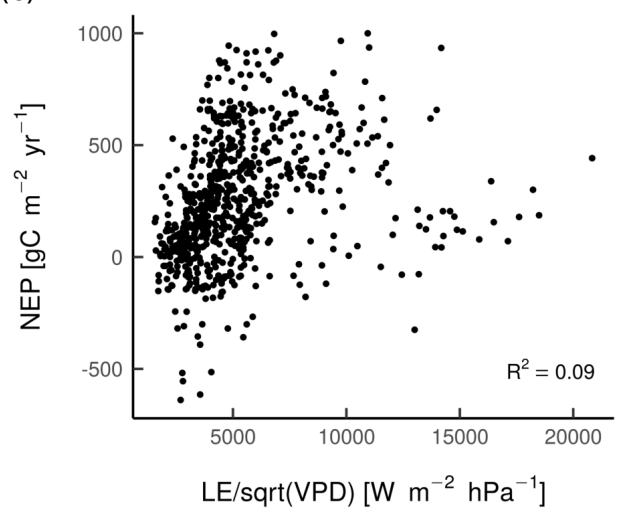

(b)

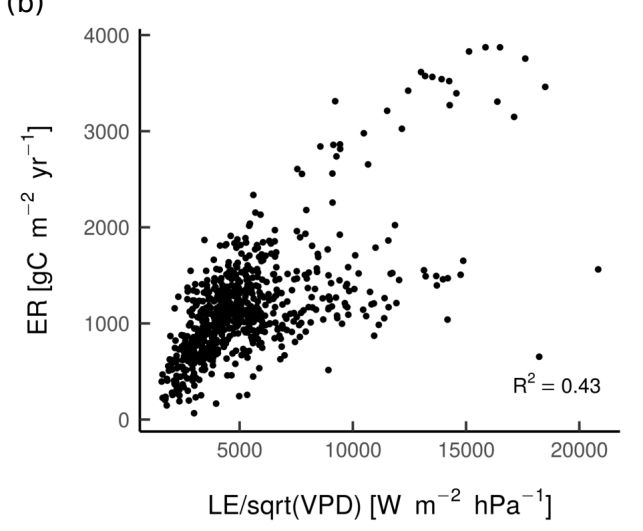

(d)

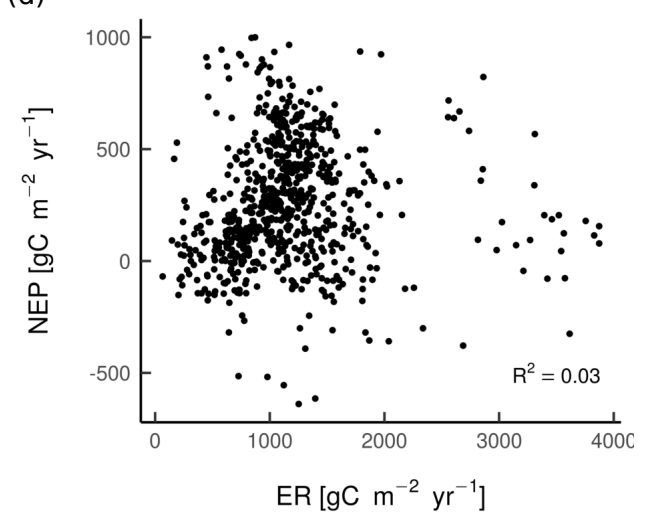

Figure 2.A3: Correlation between LE/sqrt(VPD) or GPP' and GPP (a), correlation between GPP' $^{\prime}$ and ER (b), correlation GPP' and NEP (c), and correlation between ER and NEP (d). 


\section{Residuals analysis}

(a)

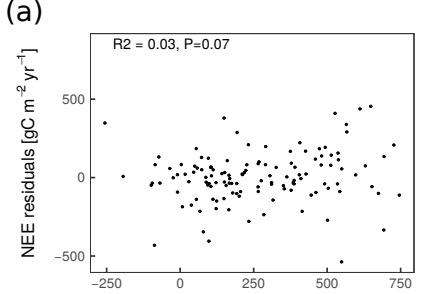

(d) NEE predicted $\left[\mathrm{gC}^{-2} \mathrm{yr}^{-1}\right]$

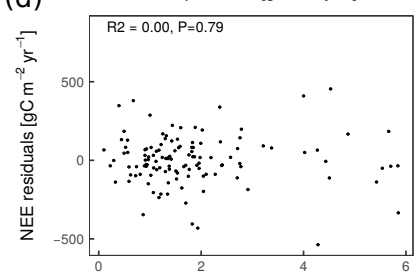

(g)

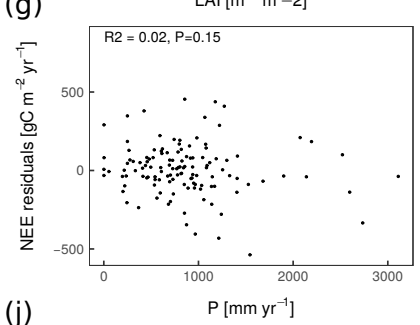

(j)

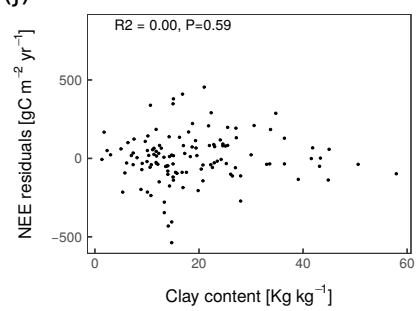

(b)

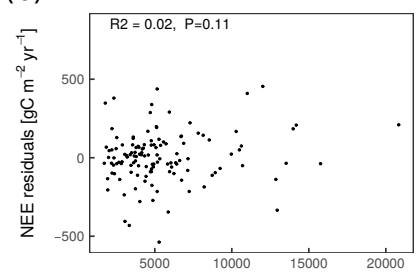

(e) LE/sqrt(VPD) $\left[\mathrm{W} \mathrm{m}^{-2} \mathrm{hPa}^{-1}\right]$

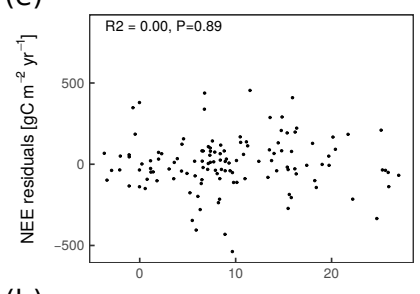

(h)

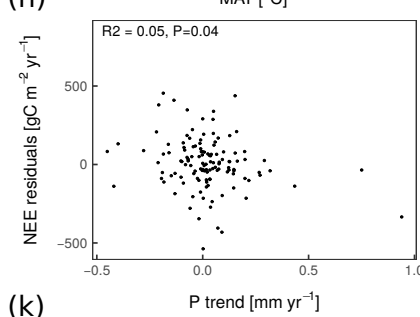

(k)

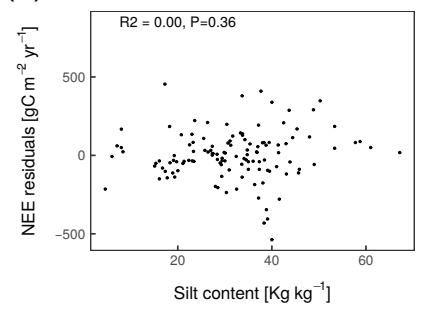

(c)

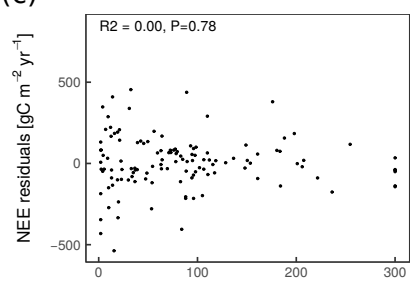

(f)

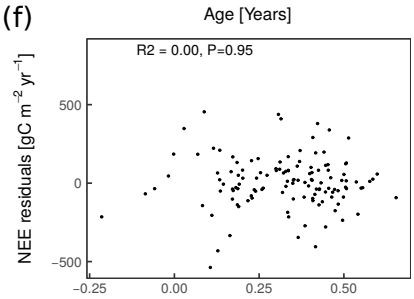

(i)

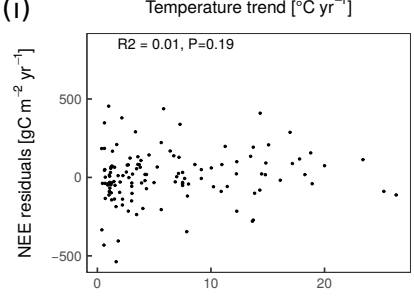

(I) $\quad \mathrm{N}$ deposition $\left[\mathrm{Kg} \mathrm{N} \mathrm{ha}^{-1} \mathrm{yr}^{-1}\right]$

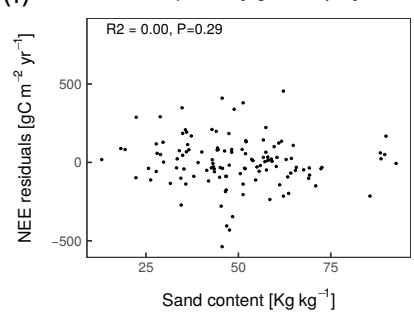

Figure 2.A4: NEP Residuals against (a) the modeled estimates and variables considered in the analysis: (b) GPP', (c) forest age, (d) LAI, (e) $\mathrm{T}_{\text {air }}$, (f) temperature trend, (g) Precip, (h) precipitation trend, (i) $\mathbf{N}_{\text {deposition }}$, (j) clay content, (k) silt content, and (l) sand content. The dots are sites $(n=126)$. 
(a)

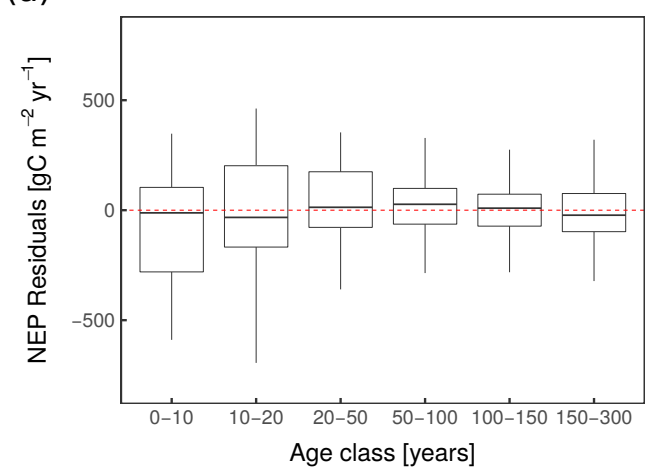

(b)

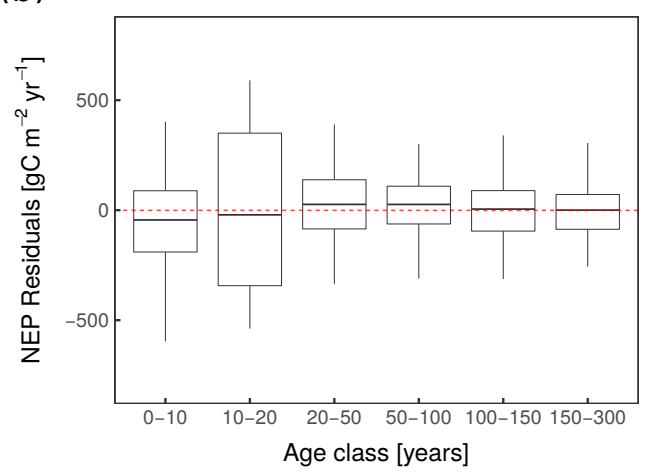

Figure 2.A5: Model residuals per age class of (a) a RF model including GPP' as a predictive variable, i.e. $\mathbf{N E P}=\mathbf{f}\left(\right.$ age $, \mathbf{g}($ age $\left.), \mathbf{G P P}^{\prime}, \mathrm{T}_{\text {air }}, \mathbf{N}_{\text {deposition }}\right)$ and (b) a RF model excluding GPP' and $\mathbf{T}_{\text {air }}$ as a predictive variable, i.e. $\mathbf{N E P}=\mathbf{f}\left(\right.$ age, $\mathbf{g}($ age $\left.), \mathbf{N}_{\text {deposition }}\right)$. 


\section{Chapter 3}

\section{The global forest carbon balance inferred from flux towers and forest age}

This chapter is based on:

P. Ciais, S. Besnard, Y. Yao, J. Chave, M. Herold, S. Piao, B. Poulter, T. Pugh, M. Reichstein, D. M. Rozendaal, P. Stoy, C. Yue, and N. Carvalhais (2019). "The global forest carbon balance inferred from flux towers and forest age". In preparation 


\section{Abstract}

Terrestrial gross primary production and energy fluxes have been successfully scaled-up from ecosystem eddy-covariance (EC) observations to the global scale. Yet, the lack of information related to site history globally (e.g. forest age) and disturbance regimes has hampered the production of realistic net ecosystem production (NEP) (i.e. the net forest carbon (C) balance including soils) global products. In fact, while forest age has been shown to be an important determinant of NEP, global diagnostic of forest demography distribution are sparse and mainly produce at regional scales. Here we employ different data-driven statistical models to translate a new forest age map and gridded inputs of environmental conditions into forest NEP global grids at $0.5^{\circ}$ spatial resolution for the period 2000-2013. With this approach, we estimate the global forest NEP as a $\mathrm{C}$ sink of ranging from +4.5 to $+5.3 \mathrm{PgC}_{\text {year }}{ }^{-1}$ with associated uncertainties ranging from 0.2 to $0.3 \mathrm{PgC}_{\text {year }}{ }^{-1}$. By removing from NEP the losses of $\mathrm{C}$ that are not observed at flux tower locations: fire emissions, harvested biomass, $\mathrm{C}$ leached to rivers, and non-carbon dioxide $\left(\mathrm{CO}_{2}\right)$ gases released to the atmosphere, we estimate the net biome production (NBP) being close to $+3 \pm 0.3 \mathrm{PgC}_{\text {year }}{ }^{-1}$. Temperate forest NBP is a $\mathrm{C}$ sink of around $+1.5 \pm 0.05-0.06$ $\mathrm{PgC}$ year $^{-1}$, almost twice larger than inferred from biomass inventories. Boreal forest NBP is a small $\mathrm{C}$ sink ranging from +0.3 to $+0.8 \pm 0.2 \mathrm{PgC}_{\text {year }}{ }^{-1}$. The NBP of tropical forests is estimated to be between $+0.4 \pm 0.2$ and $+0.7 \pm 0.2 \mathrm{PgC} \mathrm{year}^{-1}$, which is about $\frac{1}{4}$ to $\frac{1}{6}$ of that inferred from forest inventory. This first attempt to provide global estimates of the forest $\mathrm{C}$ balance inferred from EC data, statistical data-driven models, and a new global forest age map gives new insights on both the location and the magnitude of the global land $\mathrm{C}$ sink. 


\subsection{Introduction}

Most of the global land carbon (C) sink is expected to be in forest biomes (Pan et al., 2011b), however current observations are not accurate enough to represent the global land $\mathrm{C}$ sink's location. Currently, forest inventories and atmospheric inversions are used for estimating the $\mathrm{C}$ stock changes in tree biomass and the net ecosystem exchange (NEE) over large regions, respectively. While biomass inventories have good coverage of managed forests, they are very sparse both in tropical and unmanaged forests (Pan et al., 2011b). These forest inventories measure biomass stock changes from repeated sampling campaigns, but changes in soil C stocks are usually modeled. Another approach to estimate the global land $\mathrm{C}$ sink is from global maps of carbon dioxide $\left(\mathrm{CO}_{2}\right)$ fluxes obtained from top-down atmospheric inversions, however this approach provides too coarse spatial resolution (Peylin et al., 2013) for precisely separating forests from other biomes. Furthermore, such techniques are very sensitive to transport model errors (Stephens et al., 2007). Based on global eddy-covariance (EC) networks (Baldocchi, 2008; Baldocchi et al., 2001), data-driven approaches have paved the way to characterize the spatiotemporal variations of $\mathrm{CO}_{2}$ and surface energy fluxes (Jung et al., 2011; Tramontana et al., 2016), providing a promising avenue to estimate the global terrestrial $C$ sink despite the uneven coverage of the EC network. However, realistic estimates of the net ecosystem production (NEP) $(\mathrm{NEP}=-\mathrm{NEE})$ inferred from EC measurements have never been obtained. For instance, the global mean NEP up-scaled from flux towers was found to be of $+18.41 \pm 2.08 \mathrm{PgC} \mathrm{year}^{-1}$ (Zscheischler et al., 2017), which is a spatially too large $C$ sink. As a consequence, the gap has not been closed between site-scale EC observation and global patterns of forests $\mathrm{C}$ fluxes.

One of the reasons why these up-scaled NEP products (Jung et al., 2011; Tramontana et al., 2016) depict an unreasonably large $C$ sink (Zscheischler et al., 2017) could rely on the fact that they neither consider proxies for disturbance history (e.g. forest age) nor explicitly represent our ecological understanding of the forest age-NEP spatiotemporal dynamics. In fact, age since disturbance is a primary covariate related to annual local-scale NEP in forest ecosystems (Besnard et al., 2018) (Chapter 2). Yet, to account for the effect of forest age on NEP from site-level to the global scale, information on the geographical distribution of forest demography is required, as well as a precise characterization of how NEP varies with age. Another difficulty is the biased sampling of the global EC networks in the age-dimension. FLUXNET (https://fluxnet.fluxdata.org) (Baldocchi, 2008; Baldocchi et al., 2001), which is the most geographically representative EC network, contains many young to mid-age growing forests (i.e. forest age $>20$ years old), which are strong $\mathrm{C}$ sinks. On the other hand, FLUXNET network has only a few freshly disturbed forests (i.e. forest age $<20$ years old), which are expected to be net sources of $\mathrm{CO}_{2}$ to the atmosphere because of the decomposition of coarse woody debris, litter, and soil organic matter (measured as heterotrophic respiration (Rh)) that exceeds gross primary productivity (GPP) (Harmon et al., 1990; Pregitzer and Euskirchen, 2004; Wirth et al., 2002), as well as very few old forests (i.e. forest age >100 years old) that can be C neutral, sinks or sources (Luyssaert et al., 2008; Odum, 1969). As such, by not explicitly 
constraining data-driven statistical models with disturbance history or forest age-NEP dynamics but only with climate and remote-sensing variables, net $\mathrm{CO}_{2}$ fluxes in regions with freshly disturbed forests and old growth forests may not be realistically estimated.

In this study, we aim to present new evidence on both the location and the magnitude of forest C sinks globally for the period 2001-2013. Based on EC data of 119 forest FLUXNET sites, local meteorological data, and site-level forest age information, we develop a series of statistical models to estimate forest NEP at the global scale by considering not only the environmental conditions but also the effect of forest age on NEP. Additionally, by removing local C fluxes to the atmosphere, that are not measured by flux towers, from NEP global estimates, we estimate forest global net biome production (NBP). In particular, this study focuses on: 1) creating a new gridded map of forest age inferred from forest inventory data, climate variables, and fire products, 2) producing spatial forest NEP maps at $0.5^{\circ}$ to describe the location/magnitude of forest $\mathrm{C}$ sinks globally, and 3) providing new estimates of forest global NEP and NBP.

\subsection{Material and methods}

\subsubsection{Site level data}

We used a global dataset of 119 sites and 688 site-years of NEP measurements from FLUXNET forest sites ranging from 1 to more than 150-year-old stands (Table 3.A1). The sites were part of both version- 2 of the La Thuile FLUXNET and the FLUXNET2015 datasets (https: //fluxnet.fluxdata.org) of the FLUXNET network (Baldocchi, 2008; Baldocchi et al., 2001). We aggregated half-hourly NEP, GPP, and microclimatic variables (i.e. air temperature $\left.\left(\mathrm{T}_{\text {air }}\right)\right)$ at an annual scale. Data processing of EC data included storage-correction, despiking, $\mathrm{u}_{*}$-filtering (Papale et al., 2006), flux partitioning (Reichstein et al., 2005). Only sites with more than $80 \%$ of the original or good quality gap-filled data were included. The annual NEP estimates of four wet tropical sites (i.e. BR-Ma2, PA-Spn, AU-How, MY-PSO) were retrieved from the original publications (Araújo et al., 2002; Beringer et al., 2007; Kosugi et al., 2012; Wolf et al., 2011), for which higher $\mathrm{u}_{*}$-filtering thresholds than in the FLUXNET datasets (e.g. AU-How $=0.15 \mathrm{~m} \mathrm{~s}^{-1}$ or PA-Spn $=0.02 \mathrm{~m} \mathrm{~s}^{-1}$ during the dry season, $0.01 \mathrm{~m} \mathrm{~s}^{-1}$ during the wet season, and $0.05 \mathrm{~m} \mathrm{~s}^{-1}$ during the dry-wet transition) were used to correct for low-turbulence events during nighttime, therefore to decrease uncertainty in the magnitude of nocturnal fluxes. Site-level forest age data were derived from a published dataset (Besnard et al., 2018) (Chapter 2) (Fig. 3.A1), while local total atmospheric nitrogen deposition $\left(\mathrm{N}_{\text {deposition }}\right)$ estimates were collected from the biological, ancillary, disturbance and metadata database (BADM) or from the gridded emissions dataset (Wang et al., 2017) when not available at FLUXNET site level. 


\subsubsection{Description of the different statistical models}

\section{Age-Climate approaches}

The statistical models of the age-climate approaches (AC) followed Besnard et al. (2018) (Chapter 2). Here, a Random Forest (RF) model (Breiman, 2001; Kuhn, 2008) was developed to estimate the spatiotemporal variability of NEP, where NEP was given by:

$$
N E P=f\left(\text { age }, g(\text { age }), G P P, T_{\text {air }}, N_{\text {deposition }}\right)
$$

g(age) represented a non-linear transformation of the age effect on NEP. Both Tang et al. (2014) and Amiro et al. (2010) non-linear models were used to create two g(age) functions. As such, an AC approach using the Tang et al. (2014) model (hereafter AC-Tang) and an AC approach using the Amiro et al. (2010) model (hereafter AC-Amiro) were presented.

\section{Climate approach}

The aforementioned AC approaches were benchmarked against the climate only approach (hereafter Clim). This approach was similar to the AC approaches but the forest age variables (i.e. age and $g($ age $)$ ) were removed from the statistical model. NEP was given as follows:

$$
N E P=f\left(G P P, T_{\text {air }}, N_{\text {deposition }}\right)
$$

The performance of these three statistical models (i.e. AC-Amiro, AC-Tang, and Clim) was evaluated using a leave-one-site-out cross-validation approach (Fig. 3.1). We excluded one site at a time in every training set to predict the annual variations in NEP at the excluded site. The statistics used to analyze the results included the Nash-Sutcliffe model efficiency (NSE) (Nash and Sutcliffe, 1970) and root mean square error (RMSE) (Omlin and Reichert, 1999).

\subsubsection{Global gridded products}

\section{Forest age datasets}

The globally gridded forest age dataset was developed by collecting plot level stand age and aboveground biomass (AGB) estimates (12,395 plots for 28,000 measurements) (Fig. 3.A2) from a series of forest inventory databases (Álvarez-Dávila et al., 2017; Anderson-Teixeira et al., 2018; Anderson-Teixeira et al., 2016; Baker et al., 2016; Johnson et al., 2016; Lewis et al., 2013; Mitchard et al., 2014; N'Guessan et al., 2019; Poorter et al., 2016; Schepaschenko et al., 2017; Somogyi et al., 2008; Sullivan et al., 2017). For each forest inventory plot, remote-sensing observations of tree cover (Hansen et al., 2013), Landsat annual composite 
of both normalised difference vegetation index (NDVI) and normalised difference moisture index (NDMI) (https://landsat.usgs.gov/), soil properties (Wieder, 2014), and climate data (Fick and Hijmans, 2017) were extracted. For each plot, potential global radiation $\left(\mathrm{R}_{\text {pot }}\right)$ was also calculated. From these aforementioned set of predictors, we performed a feature selection procedure based on the Guided Hybrid Genetic Algorithm (Jung and Zscheischler, 2013). The best set of covariates selected by the feature selection algorithm (i.e. AGB, a series of climate variables, and $\mathrm{R}_{p o t}$ ) was further used to upscale in-situ forest age estimates to the global scale at $1 \mathrm{~km}$ resolution by using a RF algorithm (Breiman, 2001) (number of trees $=100$, minimum leaf size $=5$ ). Climate grids were collected from the WorldClim dataset (Fick and Hijmans, 2017) while a series of AGB grids (i.e. corrected for tree cover with thresholds of 5\%, 10\%, 15\%, 20\%, and 30\%) were collected from the Globbiomass project (http://globbiomass.org/). As such, five forest age maps circa 2010 were obtained from several AGB maps (i.e. Globbiomass products) using different tree cover thresholds.

Additionally, we created a forest age product inferred from the MCD45A1 MODerate-resolution Imaging Spectroradiometer (MODIS) fire product at $1 \mathrm{~km}$ resolution (Giglio et al., 2018). For the period 2000-2015, forest age was determined as the last time since a fire event occurred within a gridcell. For example, forest age within a $1 \mathrm{~km}$ gridcell was 5 years old if the last time a fire occurred within this gridcell was in 2010. The forest age estimates derived from the MODIS fire product took precedence over the forest age estimates derived from climate variables and AGB within each gridcell because the uncertainty of the MODIS fire product was assumed to be low. As such, we assumed that the reported fire events were intense enough to kill the vast majority of trees within a $1 \mathrm{~km}$ gridcell. From the $1 \mathrm{~km}$ resolution forest age maps, we further created maps that reflected the fraction of several age classes (0-30 with annual resolution and 30-150 with decadal resolution) within each $0.5^{\circ}$ gridcell (hereafter MPI-age).

\section{Gross primary production datasets}

We used nine empirical, machine learning based products from FLUXCOM (www . fluxcom. org) for GPP. The FLUXCOM products based on remote sensing and climate based predictor variables ("FLUXCOM-RS+meteo"; see Tramontana et al. (2016)) were considered. The different GPP members were created by using three different machine learning methods (i.e. a RF, multivariate regression splines, and an artificial neural network), three different climate forcings (i.e. CERES, CRUNCEP version 6, and WFDEI), and the Reichstein et al. (2005) partitioning method. For each GPP member, annual sums for the period 2001 to 2013 were calculated. For the purpose of the study, we only used the "FLUXCOM-RS+meteo" products (i.e. not considering "FLUXCOMRS" products) that were partitioned per plant functional type (PFT), therefore enabled the creation of GPP gridded products strictly limited to forest ecosystems. 
Air temperature and nitrogen deposition datasets

$\mathrm{T}_{\text {air }}$ grid product was collected from the CRUNCEP datasets (Viovy, 2018) while $\mathrm{N}_{\text {deposition }}$ grid was collected from the gridded emissions dataset (Wang et al., 2017). For both gridded products, annual means for the period 2001-2013 were computed.

From the AC-* and the Clim approaches, we translated the aforementioned set of gridded products (i.e. forest age, GPP, $\mathrm{T}_{\text {air }}$, and $\mathrm{N}_{\text {deposition }}$ spatial grids) into forest NEP products at $0.5^{\circ}$ resolution representing the period 2000-2013. To estimate NEP including spatial uncertainty, we used all the possible combinations of GPP, $\mathrm{T}_{\text {air }}$, forest age, and $\mathrm{N}_{\text {deposition }}$ members to create an integrated ensemble of NEP $\left(\mathrm{N}_{\text {member }}=9\right.$ GPP x 5 forest age x $1 \mathrm{~T}_{\text {air }} \times 1 \mathrm{~N}_{\text {deposition }}=45$ members). The final NEP products resulted from the median estimate of the different members $(\mathrm{N}=45)$ while uncertainty was defined as the inter-quantile range across all members.

\subsubsection{Estimations of net biome production}

NBP was calculated by removing from the up-scaled NEP the local C fluxes to the atmosphere that were not measured by flux towers (i.e. fire emissions and reduced $\mathrm{C}$ gases emissions), the $\mathrm{C}$ fluxes that correspond to a lateral transport followed by oxidation and $\mathrm{CO}_{2}$ emissions (i.e. outgas emissions from rivers), and the $\mathrm{C}$ fluxes that are related to wood harvest. This is given by:

$$
N B P=N E P-E_{\text {fire }}-E_{\text {reduced }}-E_{\text {river outgas }}-E_{\text {harvest }}
$$

Where $\mathrm{E}_{\text {fire }}$ was the $\mathrm{C}$ loss from forest fires to the atmosphere, $\mathrm{E}_{\text {reduced }}$ was the emissions of reduced $\mathrm{C}$ gases such as volatile organic carbon (VOC), $\mathrm{E}_{\text {river outgas }}$ was the outgassing of $\mathrm{CO}_{2}$ related to the forest's dissolved organic carbon (DOC) in lakes and rivers as well as the transport of DOC to oceans, and $\mathrm{E}_{\text {harvest }}$ was the removal of timber and fuelwood that is released as $\mathrm{CO}_{2}$ outside forest ecosystems when the wood is used by human activities.

\section{Forest fire carbon emissions}

We used the GFED4s global fire emissions data set (Werf et al., 2017) (http://www. globalfiredata.org/) to calculate forest fire emissions. We included emissions from temperate and boreal forest fires as well as tropical deforestation fires and tropical woodland fires. An uncertainty of $50 \%$ for all fire types of fire emissions was assumed (Werf et al., 2017).

\section{Reduced carbon emissions}

We used the global gridded vegetation VOC emissions simulated by the CLM/MEGANv2.1 model (http://lar.wsu.edu/megan/guides.html) at $1^{\circ}$ spatial resolution. The European 
Space Agency (ESA) land cover climate change initiative (CCI) map (Li et al., 2018) was used to retrieve the relative fraction of forest area within at $1^{\circ}$ gridcell in order to attribute VOC emissions strictly to forest ecosystems.

\section{River $\mathrm{CO}_{2}$ outgassing derived from forest DOC leaching}

For the river export and river outgassing, we used the data provided by Lauerwald et al. (2015) for the different REgional Carbon Cycle Assessment and Processes (RECCAP) regions. Similar to the VOC emissions, the ESA land cover CCI map (Li et al., 2018) was used to provide an homogeneous source of fluxes through river export and outgassing across ecosystems to be scaled by forest area.

\section{Wood harvest fluxes}

Wood harvest data including both timber wood and fuelwood extraction were collected from the Food and Agriculture Organization of the United Nations (FAO) forest resource assessment (FRA). To attribute wood harvest fluxes into biomes of boreal, temperate, and tropical forests, we first generated a gridded wood harvest data from FRA national wood harvest information and then overlapped the gridded data with the Köppen Geiger A2 Scenario 2001-2025 biome distribution map. We used a global forest coverage map from Schepaschenko et al. (2015) that is consistent with the FAO national forest area and assumed that gridcell wood harvest volume was proportional with its forest area. We used three different reports of the wood harvest volume for 2005 from FRA2005 (FAO, 2006), FRA2010 (FAO, 2010), and FRA2015 (FAO, 2015) to generate the mean value and standard deviation of wood harvest volumes for each biome. To convert harvest volume information to $\mathrm{C}$ fluxes, we used a wood density of $0.6 \mathrm{Mg} \mathrm{C} \mathrm{m}^{-3}$ and we assumed dry biomass was $50 \% \mathrm{C}$ (Houghton and Nassikas, 2017). Note that the wood harvest flux estimates solely include the stocks of $\mathrm{C}$ removed but not the downstream fluxes resulting from decomposition that can change according to harvest practices.

\subsection{Results and discussion}

\subsubsection{Model performance of the statistical models}

The capacity of the three statistical models (i.e. AC-Amiro, AC-Tang, and Clim) to reproduce NEP was assessed in a leave-one-site out cross-validation (Fig. 3.1). As expected, the Clim approach had a worse predictive capacity (NSE $=0.25$ and $\mathrm{RMSE}=257.01 \mathrm{gC} \mathrm{m}^{-2} \mathrm{year}^{-1}$ ) compared to the AC approaches, with the AC-Amiro approach showing the best model performance $\left(\mathrm{NSE}=0.63\right.$ and $\mathrm{RMSE}=179.75 \mathrm{gC} \mathrm{m}^{-2}$ year $^{-1}$ ) followed by the AC-Tang approach (NSE= 0.44 and $\mathrm{RMSE}=217.17 \mathrm{gC} \mathrm{m}^{-2}$ year $^{-1}$ ). This finding confirmed the importance of considering 
forest age when explaining NEP spatiotemporal variability at FLUXNET site level (Besnard et al., 2018; Chen et al., 2003; Chen et al., 2002; Coursolle et al., 2012; Gao et al., 2016; Yu et al., 2014). Although the presented approaches were able to reproduce the NEP spatiotemporal variability, particularly the AC-Amiro approach, significant residuals were apparent for individual FLUXNET sites (Fig. 3.A2). This suggested that the proposed statistical methods were not able to capture some of the local variabilities that could be related to site-specific environmental conditions and history as well as related to the quality of the EC data. Yet, the model performance of the statistical models encouraged the upscaling of NEP based on environmental, GPP, and forest age global spatial grids.
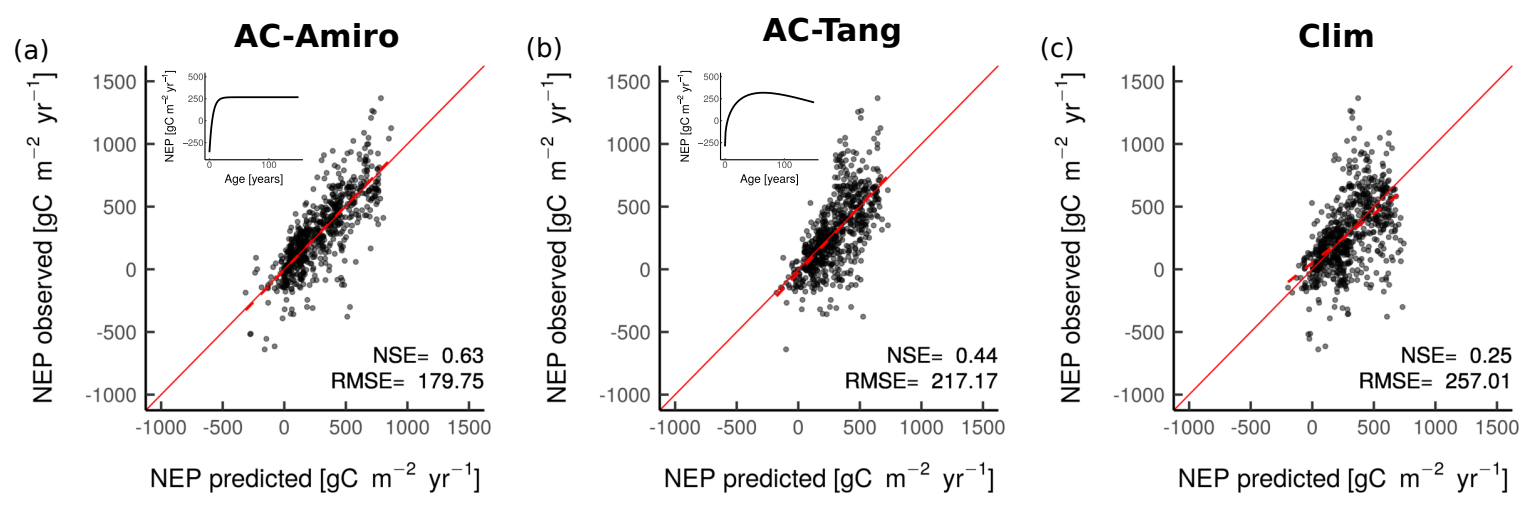

Figure 3.1: Cross-validated comparison of predicted vs. observed NEP estimates for the ACAmiro (a), AC-Tang (b), and Clim approaches (c). The inner plots in (a) and (b) show the age-NEP non-linear models.

\subsubsection{Assessment of the presented forest age maps}

While global climate, GPP, and $\mathrm{N}_{\text {deposition }}$ datasets have been largely developed over the past decades, very few products describing the geographical distribution of forest age globally are available (Poulter et al., 2019). This lack of information on forest demography limited our capacity to provide estimates of NEP spatiotemporal variabilities more realistically up to now. By providing a new global forest age map, we demonstrated the large variability of forest age across the globe (Fig. 3.2). The area occupied by very young forests reflected recent standreplacing disturbances or afforestation/reforestation practices. China depicted relatively young forests due to the implementation of afforestation policies as well as natural disturbances (e.g. fire) (Zhang et al., 2017). Recently disturbed forests can also be found in the African tropical dry forest, where the frequency of the fire regimes is very high (Werf et al., 2017). Large scale fires in the boreal region also resulted in widespread patches of young forests, while European forests (mainly managed forests) seemed to be in young/intermediate stages of forest succession. Not surprisingly, most of the old-growth/undisturbed forests ( $>150$ years old) can be found in the Amazon and the Congo basins. The spatial patterns of the MPI-age dataset were compared with a series of independent forest age products (Chazdon et al., 2016; Loboda and Chen, 2017; Moreno 
et al., 2017; Pan et al., 2011a; Poulter et al., 2019; Zhang et al., 2017) (Fig. 3.A6). In Russia, the MPI-age dataset had higher age estimates compared to the Loboda and Chen (2017) dataset, which is mainly due to the fact that the latter product only reported young forests (i.e. forest age $<30$ years old). In the Amazon, we found that most of the differences between the MPI-age product and the Chazdon et al. (2016) datasets occurred in the southern part of the Amazon. Such disagreement could be related not only to the different methods used to infer forest age regionally (i.e. statistical method vs. age-AGB chronosequence approach) but also whether one applied a tree cover correction to the AGB products or not. Similarly, the presented product and the Pan et al. (2011a) dataset revealed widespread discrepancies in the North American region. The fact that the two methods used different data streams to create their respective forest age spatial grids (i.e. AGB/climate vs. optical remote-sensing) could explain such differences. Additionally, forest inventory plots used to derive the MPI-age map were relatively sparse in Canada, which might also explain these differences. The Moreno et al. (2017) dataset seemed to have higher forest age estimates over Europe than the MPI-age product, which could be related to a relatively coarse forest age classification used in the former dataset (i.e. 0-140 years old with a bi-decadal frequency). Finally, the forest age estimates of the MPI-age product in China were rather consistent with the Zhang et al. (2017) dataset, while we found large and widespread discrepancies between the MPI-age dataset and the global forest age dataset (GFAD) (Poulter et al., 2019). Because the GFAD used a different AGB product (i.e. no tree cover correction) for the pan-tropical region and mainly relied on coarse national forest inventory data for the Northern hemisphere, widespread differences were expected between the GFAD and the MPI-age global maps.

The uncertainties in the presented forest age map were estimated by using different AGB gridded products (i.e. Globbiomass products) with different tree cover thresholds (Fig. 3.A5). Therefore, we assumed that the uncertainties in the forest inventory data and the climate data were negligible compared to the ones in AGB. The sensitivity analysis showed that regions, such as the dry tropics and Europe, had relatively high uncertainty with regard to the tree cover threshold used (e.g. no tree cover correction vs. 30\% tree cover correction). The fact that there is mosaic vegetation in the dry tropics (forest/grassland/shrubland) and in Europe (forests/croplands) within a $1 \mathrm{~km}$ gridcell may explain the sensitivity of the forest age estimates to tree cover thresholds in such regions. Furthermore, the presented forest age's spatial patterns and uncertainty estimates were dependent on the capacity of the statistical model to reproduce the plot-level age estimates (Fig. 3.A4). While the performance of the proposed statistical model was relatively high (Fig. 3.A4a, c, d, and e), in particular in explaining the latitudinal means, one could find biases in both young and old forests (Fig. 3.A4b). More precisely, the statistical model slightly overestimated the age estimates of young forests while it underestimated the age estimates of old forests at plot level. Such biases could potentially have implications in estimating the sign of the forest $\mathrm{C}$ balance (i.e. C source or $\mathrm{C}$ sink), in particular for young forests. 

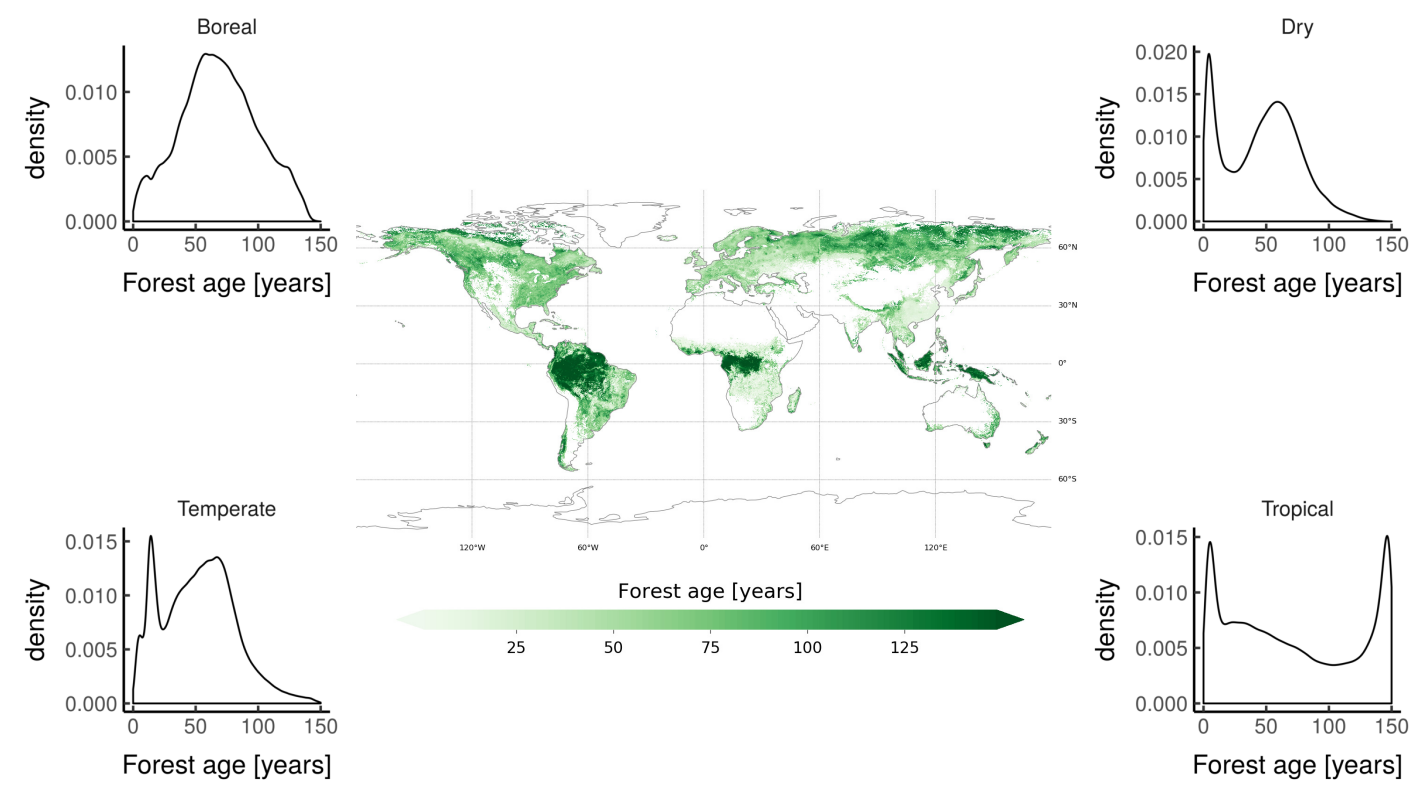

Figure 3.2: Spatial patterns of global forest age and the age distribution within four biomes: boreal, dry, temperate, and tropical. The map corresponds to a tree cover threshold of $20 \%$.

\subsubsection{Spatial patterns of NEP and forest $\mathrm{C}$ balance budgets}

Larger NEP sinks prevailed in the young and productive temperate forests of western Europe and the eastern United States (Williams et al., 2012), and in the temperate and subtropical forests of China (Fig. 3.3). In China, recent plantations resulted in young-intermediate forest structure, therefore leading to high NEP uptake (Fang et al., 2014). Boreal forests in North America and in Northern Siberia showed a net uptake in $\mathrm{CO}_{2}$, although areas showing small $\mathrm{C}$ sources could also be found in these regions. In the tropics, the proposed approach suggested that the wet forests were smaller $\mathrm{C}$ sinks per unit area than dry forests and woodlands (Fig. 3.3) despite the wet forests being much more productive. Overall, regions with forest ecosystems being a net $\mathrm{C}$ source were relatively limited across the globe (e.g. boreal forests, African dry forests). One could argue that the freshly disturbed $\mathrm{C}$ source forests were mixed with intermediate strong $\mathrm{C}$ sink forests within a $0.5^{\circ}$ gridcell. As a result, the release of $\mathrm{CO}_{2}$ to the atmosphere by freshly disturbed forests was mitigated by young and intermediate productive forests. Additionally, the aforementioned limitations of the MPI-age product (i.e. overestimation of the young forest age and underestimation of the old-growth forest age) could also have implications in the location of the $\mathrm{C}$ sources. Finally, the coarse spatial resolution of the GPP products used in this study implied that our assumptions on the forest age-GPP relationship (e.g. very young forests should correspond with low GPP) (Tang et al., 2014) was challenged, resulting in a potential overestimation of NEP for the young forests (Fig. 3.4a).

The global age-NEP dynamics somewhat confirmed our ecological understanding of the forest succession of NEP (Fig. 3.4), although here space was substituted for time. Overall, NEP was 
(a)

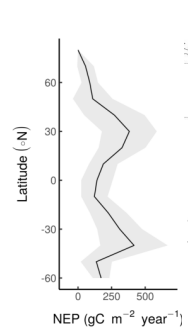

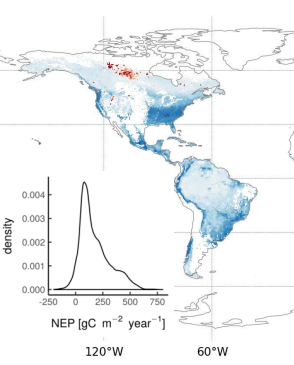

$\begin{array}{lllll}100 & 200 & 300 & 400 & 500\end{array}$

(b)

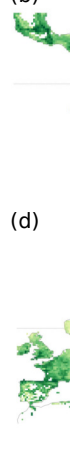

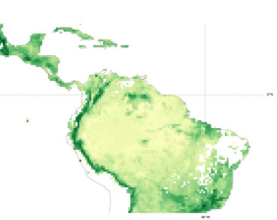

(c)

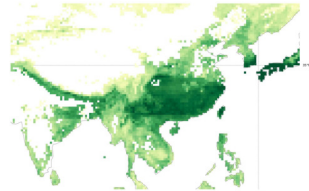

(e)

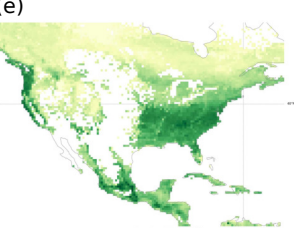

Net ecosystem production [ $\mathrm{gC} \mathrm{m}^{-2}$ year $^{-1}$ ]

$100 \quad 200 \quad 300 \quad 400 \quad 500$

Figure 3.3: Spatial distribution of NEP derived from the AC-Amiro approach (a). Focuses into he Amazon (b), China (c), Europe (d), and North America (e) regions are also shown.

lower for very young forests, followed by an increase in NEP for young- to middle-aged stands, which further declined as forests age (Fig. 3.4a). As expected, comparing the AC-Amiro and Clim approaches demonstrated that the NEP estimates for young forests were generally overestimated when the effect of forest age was not included in a statistical model (Fig. 3.4c). Interestingly, very productive tropical forest ecosystems (i.e. GPP $>2,500 \mathrm{gC} \mathrm{m}^{-2}$ year $^{-1}$ ) showed lower NEP than temperate and sub-tropical forests (i.e. GPP comprised between $1,500-2,500 \mathrm{gC} \mathrm{m}^{-2}$ year $^{-1}$ ) (Fig. 3.4). By looking at the ratio between GPP and ecosystem respiration (ER) (Fig. 3.4d), we showed that the lower NEP of very productive tropical forests was very likely related to the fact that there was a higher contribution of ER into the net $\mathrm{CO}_{2}$ fluxes for such an ecosystem compared to temperate and sub-tropical forests (i.e. lower GPP-ER ratio for forests with GPP $>2,500 \mathrm{gC} \mathrm{m}^{-2}$ year $^{-1}$ than for forests with GPP comprised between 1,500 and $2,500 \mathrm{gC} \mathrm{m}^{-2}$ year $\left.^{-1}\right)$.

Despite differences in the geographic patterns of NEP between the three presented approaches (i.e. AC-Amiro, AC-Tang, and Clim) (Fig. 3.5 and Fig. 3.A7), global NEP budgets were rather similar between the AC-Amiro $\left(+4.96 \pm 0.16 \mathrm{PgC}_{\text {year }}{ }^{-1}\right)$, AC-Tang $(+5.31 \pm 0.15 \mathrm{PgC}$ year $^{-1}$ ), and Clim $\left(+4.45 \pm 0.24 \mathrm{PgC}_{\text {year }}{ }^{-1}\right.$ ) approaches (Table 3.1). The global NEP budget calculated with an independent global forest age map (Poulter et al., 2019) (GFAD) was also comparable $\left(+4.70 \pm 0.28 \mathrm{PgC} \mathrm{year}^{-1}\right)$ with the AC-Amiro, AC-Tang, and Clim approaches. NEP budgets were aggregated into boreal, temperate, and tropical forest regions. We found large $\mathrm{C}$ sinks in tropical and temperate forests, while the boreal forest biome was only a small C sink (Table 3.1). Interestingly, we found a substantially smaller NEP estimate in the tropical forests (around $+2 \mathrm{PgC}_{\text {year }}{ }^{-1}$ ) compared to a previous estimate of tropical forest NEP based on flux towers (+5.0 $\pm 0.6 \mathrm{PgC}_{\text {year }}{ }^{-1}$ ) (Zscheischler et al., 2017). This large difference between the two estimates was not only due to the fact that forest age was explicitly accounted for in this study 


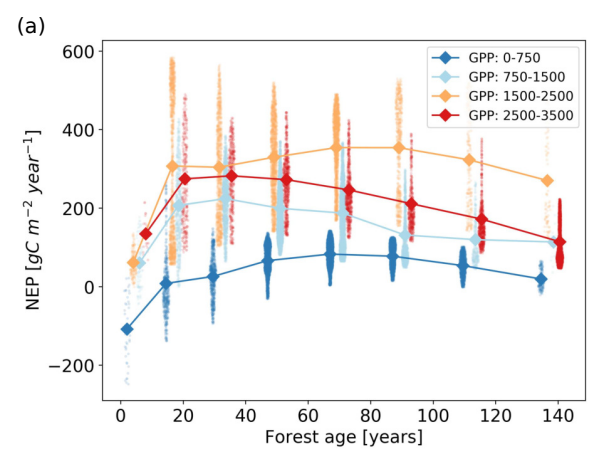

(c)

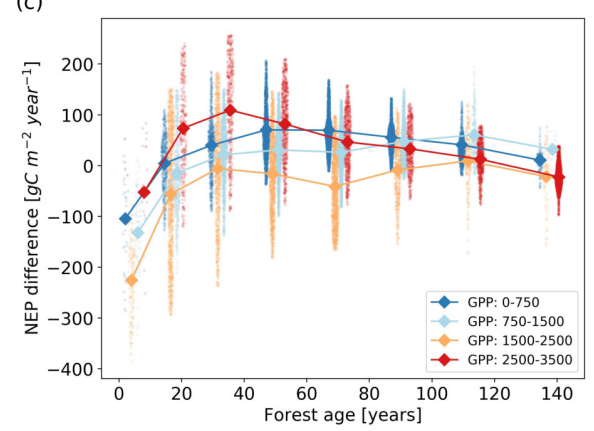

(b)

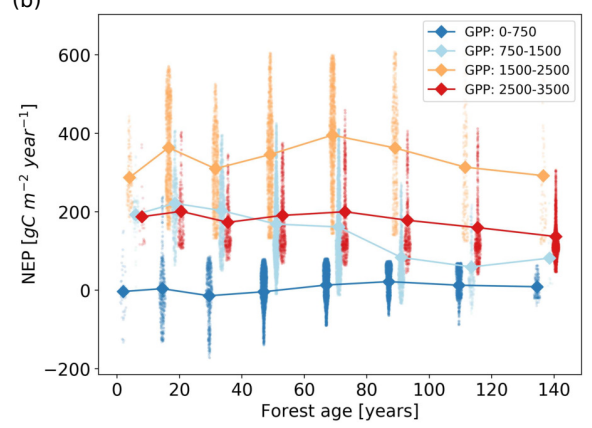

(d)

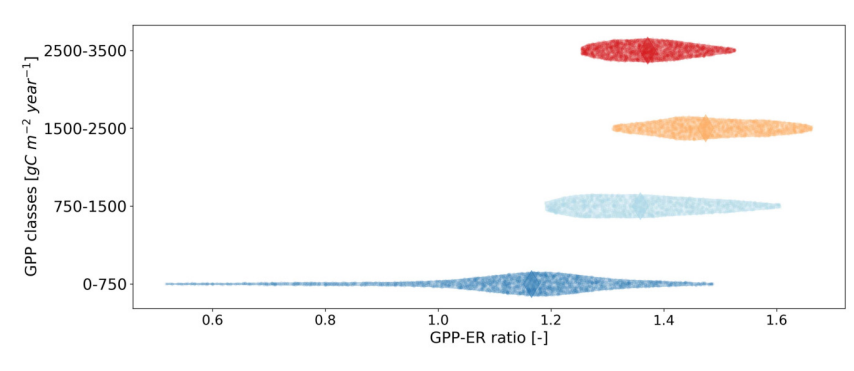

Figure 3.4: Scatterplots of NEP derived from AC-Amiro (a) and Clim (b) against forest age. The differences between NEP estimates from the AC-Amiro and the climate approaches against forest age are also shown (c). Forest age estimates were binned into 8 classes: 0-10, 0-25, 25-40, 40-60, 60-80, 80-110, 110-130, and 130-150 and GPP estimates were binned into 4 classes: 0-750, 750-1500, 1500-2500, and 2500-3500. GPP-to-ER ratio plotted per GPP bins can be found in (d).

but also because the annual NEP measurements for four FLUXNET sites in the wet tropics were corrected for low nighttime turbulence in this study. (see methods).

In order to estimate NBP, we removed from the NEP estimates the C losses that were not measured by flux towers (see methods and Table 3.A2). Our global estimates of forest NBP were net $\mathrm{C}$ sinks of $+2.78 \pm 0.23,+3.16 \pm 0.24$, and $+2.21 \pm 0.26 \mathrm{Pg} \mathrm{C}_{\mathrm{Cear}}{ }^{-1}$ for the AC-Amiro, AC-Tang, and Clim approaches, respectively (Table 3.1). These estimates were somewhat consistent with the one inferred from forest biomass inventories (Pan et al., 2011b) at the global scale. This result implied that the world forests account for nearly all the global residual land $\mathrm{C}$ sink (i.e. difference between $\mathrm{C}$ accumulated in the atmosphere and amount taken up by the global oceans), excluding land-use $\mathrm{CO}_{2}$ emissions. Yet, we found that the $\mathrm{C}$ sink of temperate forests was twice larger than what was inferred from biomass inventories. A possible explanation for this discrepancy was that the study from Pan et al. (2011b) estimated soil C storage in these forests from models, and therefore may have under-estimated this component of NBP. Conversely, we found that the $\mathrm{C}$ sink in tropical forests was about $\frac{1}{4}$ to $\frac{1}{6}$ of that derived from forest inventory (excluding land use change emissions). The sparse representation of both forest inventory plots and EC flux towers in the tropics ( $N=10$ sites and mainly old-growth forests) challenged any possible conclusions made on the magnitude of the $\mathrm{C}$ sink in these regions. When gross deforestation emissions (i.e. the total $\mathrm{C}$ emissions from tropical deforestation and 

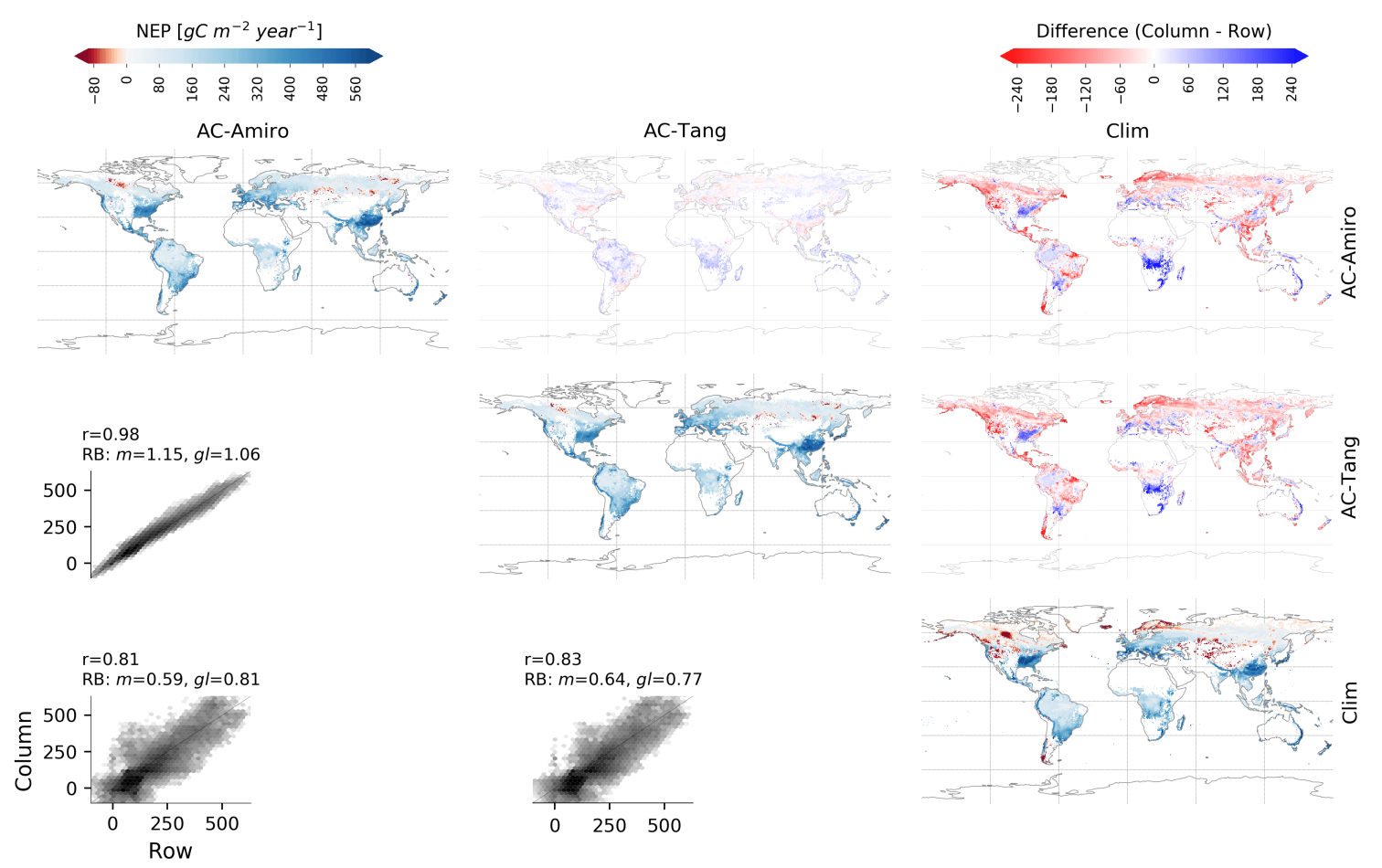

Figure 3.5: Comparision of the NEP spatial patterns between the three different approaches: AC-Amiro, AC-Tang, and Climate.

logging) (-2.94 $\pm 0.47 \mathrm{PgC} \mathrm{year}^{-1}$ ) (Pan et al., 2011b) was added to NBP in the tropical forests, the net tropical $\mathrm{C}$ balance became a $\mathrm{C}$ source of $-2.52 \pm 0.54 \mathrm{PgC}_{\text {year }}{ }^{-1}$ (AC-Amiro approach), although this estimate does not account for the uptake of $\mathrm{C}$ in tropical regrowth forests.

Table 3.1: NEP up-scaled from EC networks with global age-NEP relationships (AC-Amiro, ACTang), and without considering age effects (Clim). The NBP is derived from NEP by adding C losses not monitored by flux towers. NEP estimates for GFAD were derived from the AC-Amiro model. Units are in $\mathrm{PgC}_{\text {year }}{ }^{-1}$.

\begin{tabular}{|c|c|c|c|c|c|c|c|c|c|}
\hline & \multicolumn{4}{|c|}{ Net Ecosystem Productivity (NEP) } & \multicolumn{4}{|c|}{ Net Carbon Balance (NBP) } & \multirow{2}{*}{$\begin{array}{c}\text { NBP from biomass inventories } \\
\text { Pan et al. }(2011 b)\end{array}$} \\
\hline & AC-Amiro & AC-Tang & C & GFAD & AC-Amiro & AC-Tang & C & GFAD & \\
\hline Boreal & $+1.26 \pm 0.13$ & $+1.30 \pm 0.12$ & $+0.74 \pm 0.21$ & $+1.0098 \pm 0.18$ & $+0.77 \pm 0.15$ & $+0.81 \pm 0.15$ & $+0.25 \pm 0.23$ & $+0.52 \pm 0.20$ & $+0.50 \pm 0.10$ \\
\hline Temperate & $+1.89 \pm 0.042$ & $+1.93 \pm 0.039$ & $+1.92 \pm 0.055$ & $+1.76 \pm 0.052$ & $+1.50 \pm 0.053$ & $+1.54 \pm 0.051$ & $+1.53 \pm 0.064$ & $+1.37 \pm 0.062$ & $+0.80 \pm 0.10$ \\
\hline Tropics & $+1.78 \pm 0.091$ & $+2.048 \pm 0.082$ & $+1.76 \pm 0.092$ & $+1.90 \pm 0.20$ & $+0.38 \pm 0.21$ & $+0.65 \pm 0.20$ & $+0.36 \pm 0.21$ & $+0.50 \pm 0.27$ & $+2.80 \pm 0.70$ \\
\hline Other forests & $+0.026 \pm 0.00068$ & $+0.030 \pm 0.0011$ & $+0.026 \pm 0.0020$ & $+0.024 \pm 0.0015$ & $\mathrm{NaN}$ & $\mathrm{NaN}$ & $\mathrm{NaN}$ & $\mathrm{NaN}$ & $\mathrm{NaN}$ \\
\hline Globe & $+4.96 \pm 0.16$ & $+5.31 \pm 0.15$ & $+4.45 \pm 0.24$ & $+4.70 \pm 0.28$ & $+2.65 \pm 0.26$ & $+3.00 \pm 0.26$ & $+2.14 \pm 0.32$ & $+2.39 \pm 0.34$ & $+4.10 \pm 0.71$ \\
\hline
\end{tabular}

Additionally, we revisited the estimation of global NEP (i.e. considering all terrestrial ecosystems) based on the "FLUXCOM-RS+meteo" products. As the latter products were partitioned per PFT, we were able to incorporate the presented new forest NEP products into the FLUXCOM framework. While "FLUXCOM-RS+meteo" provided a global NEP estimate of $+24.00 \pm 3.83 \mathrm{PgC} \mathrm{year}^{-1}$, we found global NEP estimates (after correction) of around $+16.37 \pm 2.58,+17.23 \pm 2.49$, and $+16.28 \pm 2.95 \mathrm{PgC}_{\text {year }}{ }^{-1}$ for the AC-Amiro, AC-Tang, and Clim approaches, respectively. By contrasting the original "FLUXCOM-RS+meteo" global NEP estimates against the corrected estimates derived from the AC-Amiro, AC-Tang, and Clim 
approaches, we could argue that this substantial difference in global NEP (i.e. around $8 \mathrm{PgC}$ year $^{-1}$ ) would result in a much higher global net carbon exchange (NCE) estimate compared to the one presented in the Zscheischler et al. (2017) synthesis (based on the "FLUXCOM-RS" products) $\left(\mathrm{NCE}=-5.45 \pm 1.99 \mathrm{PgC}_{\mathrm{year}}{ }^{-1}\right)$. Consequently, the forest NEP products presented in this study could potentially provide a global NCE estimate that is closer to the one accurately constrained by $\mathrm{CO}_{2}$ growth rate observations ( $\mathrm{NCE}=+4.3 \pm 0.1 \mathrm{PgC}_{\text {year }}{ }^{-1}$ ) (Le Quéré et al., 2015).

Systematic errors in the presented approaches to upscale NEP were difficult to quantify and they differed between approaches. In the AC-* approaches, forest age was included as a predictor and the spatial distribution of NEP was therefore affected by the uncertainty of the forest age maps. As previously mentioned, the area of newly disturbed forests estimated from the presented forest age product was very likely underestimated as it was shown for the young forests at plot level (Fig. 3.A4). Similarly, the fraction of old-growth forest was possibly underestimated in our forest age map product. A sensitivity analysis (Fig. 3.A8) using the aforementioned series of the regional forest age maps and an independent global forest age map (GFAD) (Poulter et al., 2019) provided insights into whether forest age was a robust feature when estimating NEP at the global scale or not. Although the spatial patterns of NEP were consistent globally when using different regional forest age maps, there were some discrepancies at the local scale, particularly in the Amazon region and Russia. On the one hand, differences in Russia were expected because the Loboda and Chen (2017) product only reported young forests (i.e. forest age $<20$ years old). On the other hand, Chazdon et al. (2016) reported a higher fraction of young forests in the Amazon basin (Fig. 3.A6), which could explain the observed differences in NEP estimates. We also found widespread discrepancies between the AC-Amiro approach and the GFAD approach, in particular in the dry tropics, that could be due to the fact that the latter approach estimated forest age from age-AGB chronosequences in this region, while the former approach used a data-driven statisitcal model. Finally, the representativeness of EC flux towers limited our confidence with regard not only to the location $\mathrm{C}$ sinks presented in this study (Fig. 3.5), particularly in the tropical regions ( $\mathrm{N}=10$ FLUXNET sites), but also to the estimates of the NEP and NBP budgets both in the tropics and globally (Table 3.1).

\subsection{Conclusion}

This study brings new observation-based estimates of the global forest balance from EC networks and calls for a better sampling of the spatiotemporal forest succession by flux towers. These ageconstrained results show consistency with independent of forest inventory's estimates, although we find substantial differences at biome level. We estimate a global forest NEP and NBP of around $+5 \mathrm{PgC}_{\text {year }}{ }^{-1}$ and $+3 \mathrm{PgC}_{\text {year }}{ }^{-1}$, respectively. Although forest age is found to be a key predictor of forest $\mathrm{CO}_{2}$ fluxes, both the quality and the coverage of the FLUXNET network in the tropics are critical sources of uncertainty. Interestingly, even without including age in a 
statistical model, realistic global, and regional $\mathrm{CO}_{2}$ flux estimates could be obtained with the Clim approach, suggesting that the correction of NEP estimates for low nighttime turbulence in the tropical sites plays a significant role. The future releases of the FLUXNET dataset will provide more sites, longer time series, and higher data quality data, and therefore would enable a better evaluation of the ability of the presented statistical models to explain site-level trends and interannual variability of NEP and could also improve our confidence on the location of the $\mathrm{C}$ sink regionally and globally. 


\section{A Appendix}

This appendix represents the supplementary materials of the presented publication.

\section{Sites used in this study}

Table 3.A1: List of sites used in this study. $\mathrm{DBF}=$ Deciduous broadleaf forest, $\mathrm{DNF}=$ deciduous needleleaf forest, $\mathrm{EBF}=$ evergreen broadleaf forest, $\mathrm{ENF}=$ evergreen needleleaf forest, $\mathrm{MF}=$ mixed forest. The PFT and climate classifications used here are coming from the ancillary data files provided by the La Thuile or the FLUXNET2015 datasets (https://fluxnet.fluxdata.org).

\begin{tabular}{cccccc}
\hline Site ID & Lat $\left[{ }^{\circ} \mathrm{N}\right]$ & Long $\left[{ }^{\circ} \mathrm{E}\right]$ & Climate type & Vegetation type & Forest age \\
& & & & & \\
\hline AR-SLu & -33.4648 & -66.4598 & Temperate & MF & 50 \\
AR-Vir & -28.2395 & -56.1886 & Temperate & ENF & 8 \\
AU-Cum & -33.6133 & 150.7225 & Temperate & EBF & $>150$ \\
AU-Rob & -17.1175 & 145.6301 & Temperate & EBF & 198 \\
AU-Tum & -35.6566 & 148.1517 & Temperate & EBF & 83 \\
AU-Wac & -37.4259 & 145.1878 & Temperate & EBF & $>150$ \\
AU-Wom & -37.4222 & 144.0944 & Temperate & EBF & 32 \\
BE-Bra & 51.3092 & 4.5206 & Temperate & MF & 78 \\
BE-Jal & 50.5639 & 6.0733 & Temperate & MF & 8 \\
BE-Vie & 50.3051 & 5.9981 & Temperate & MF & 94 \\
BR-Ban & -9.8244 & -50.1591 & Tropical & EBF & $>150$ \\
BR-Cax & -1.7197 & -51.4590 & Tropical & EBF & 96 \\
BR-Ji2 & -10.0832 & -61.9309 & Tropical & EBF & $>150$ \\
BR-Ma2 & -2.6091 & -60.2093 & Tropical & EBF & $>150$ \\
BR-Sa1 & -2.8567 & -54.9589 & Tropical & EBF & $>150$ \\
BR-Sa3 & -3.0180 & -54.9714 & Tropical & EBF & $>150$ \\
CA-Ca1 & 49.8672 & -125.3340 & Temperate & ENF & 60 \\
CA-Ca2 & 49.8705 & -125.2910 & Temperate & ENF & 3 \\
CA-Ca3 & 49.5346 & -124.9000 & Temperate & ENF & 16 \\
CA-Gro & 48.2167 & -82.1556 & Boreal & MF & 78 \\
CA-Man & 55.8796 & -98.4808 & Boreal & ENF & 161 \\
CA-NS1 & 55.8792 & -98.4839 & Boreal & ENF & 154 \\
CA-NS2 & 55.9058 & -98.5247 & Boreal & ENF & 73 \\
CA-NS3 & 55.9117 & -98.3822 & Boreal & ENF & 39 \\
CA-NS4 & 55.9144 & -98.3806 & Boreal & ENF & 39 \\
CA-NS5 & 55.8631 & -98.4850 & Boreal & ENF & 23 \\
CA-Oas & 53.6289 & -106.1978 & Boreal & DBF & 80 \\
CA-Obs & 53.9872 & -105.1178 & Boreal & ENF & 112 \\
CA-Ojp & 53.9163 & -104.6920 & Boreal & ENF & 88 \\
CA-Qcu & 49.2671 & -74.0365 & Boreal & ENF & 4 \\
CA-Qfo & 49.6925 & -74.3421 & Boreal & ENF & 102 \\
CA-SF1 & 54.4850 & -105.8176 & Boreal & ENF & 28 \\
CA-SF2 & 54.2539 & -105.8775 & Boreal & ENF & 14 \\
\hline & & & $C o n t i n$ & & \\
& & & & & \\
\hline
\end{tabular}

Continued on next page 
Table 3.A1 - Continued from previous page

\begin{tabular}{|c|c|c|c|c|c|}
\hline Site ID & Lat $\left[{ }^{\circ} \mathrm{N}\right]$ & Long $\left[{ }^{\circ} \mathrm{E}\right]$ & Climate type & Vegetation type & Forest age \\
\hline CA-SJ1 & 53.9080 & -104.6560 & Boreal & ENF & 10 \\
\hline CA-SJ2 & 53.9450 & -104.6490 & Boreal & ENF & 2 \\
\hline CA-SJ3 & 53.8758 & -104.6450 & Boreal & ENF & 30 \\
\hline CA-TP1 & 42.6609 & -80.5595 & Boreal & ENF & 9 \\
\hline CA-TP2 & 42.7744 & -80.4588 & Boreal & ENF & 73 \\
\hline CA-TP3 & 42.7068 & -80.3483 & Boreal & ENF & 37 \\
\hline CA-TP4 & 42.7102 & -80.3574 & Boreal & ENF & 70 \\
\hline CA-TPD & 42.6353 & -80.5577 & Boreal & DBF & 98 \\
\hline CH-Dav & 46.8153 & 9.8559 & Temperate & ENF & 222 \\
\hline CH-Lae & 47.4781 & 8.3650 & Temperate & $\mathrm{MF}$ & 184 \\
\hline CN-Anh & 33.0000 & 117.0000 & Temperate & $\mathrm{DBF}$ & 13 \\
\hline CN-Bed & 39.5306 & 116.2520 & Boreal & $\mathrm{EBF}$ & 12 \\
\hline CN-Cha & 42.4025 & 128.0958 & Boreal & $\mathrm{MF}$ & $>150$ \\
\hline CN-Din & 23.1733 & 112.5361 & Temperate & $\mathrm{EBF}$ & 96 \\
\hline CN-Hny & 29.3100 & 112.5100 & Temperate & $\mathrm{DBF}$ & 2 \\
\hline $\mathrm{CN}-\mathrm{Ku} 1$ & 40.5383 & 108.6940 & Arid & $\mathrm{EBF}$ & 5 \\
\hline CN-Qia & 26.7414 & 115.0581 & Temperate & ENF & 19 \\
\hline CZ-BK1 & 49.5021 & 18.5369 & Boreal & ENF & 31 \\
\hline DE-Bay & 50.1419 & 11.8669 & Temperate & ENF & 54 \\
\hline DE-Hai & 51.0792 & 10.4530 & Temperate & $\mathrm{DBF}$ & 254 \\
\hline DE-Har & 47.9344 & 7.6010 & Temperate & ENF & 42 \\
\hline DE-Lkb & 49.0996 & 13.3047 & Temperate & ENF & 2 \\
\hline DE-Lnf & 51.3282 & 10.3678 & Temperate & DBF & 117 \\
\hline DE-Meh & 51.2753 & 10.6555 & Temperate & $\mathrm{MF}$ & 2 \\
\hline DE-Obe & 50.7836 & 13.7196 & Temperate & ENF & 76 \\
\hline DE-Tha & 50.9636 & 13.5669 & Temperate & ENF & 118 \\
\hline DE-Wet & 50.4535 & 11.4575 & Temperate & ENF & 54 \\
\hline DK-Sor & 55.4859 & 11.6446 & Temperate & $\mathrm{DBF}$ & 85 \\
\hline ES-ES1 & 39.3460 & -0.3188 & Temperate & ENF & 116 \\
\hline FI-Hyy & 61.8475 & 24.2950 & Boreal & ENF & 46 \\
\hline FI-Sod & 67.3619 & 26.6378 & Boreal & ENF & 161 \\
\hline FR-Fon & 48.4764 & 2.7801 & Temperate & DBF & 150 \\
\hline FR-Hes & 48.6742 & 7.0646 & Temperate & $\mathrm{DBF}$ & 37 \\
\hline FR-LBr & 44.7171 & -0.7693 & Temperate & ENF & 34 \\
\hline FR-Pue & 43.7414 & 3.5958 & Temperate & $\mathrm{EBF}$ & 64 \\
\hline GF-Guy & 5.2788 & -52.9249 & Tropical & $\mathrm{EBF}$ & $>150$ \\
\hline IL-Yat & 31.3450 & 35.0515 & Arid & ENF & 39 \\
\hline IS-Gun & 63.8333 & -20.2167 & Temperate & DBF & 7 \\
\hline IT-Bon & 39.4778 & 16.5347 & Temperate & ENF & 36 \\
\hline IT-Col & 41.8494 & 13.5881 & Temperate & $\mathrm{DBF}$ & 180 \\
\hline IT-Cp2 & 41.7043 & 12.3573 & Temperate & $\mathrm{EBF}$ & 63 \\
\hline IT-Cpz & 41.7052 & 12.3761 & Temperate & $\mathrm{EBF}$ & 56 \\
\hline IT-LMa & 45.5813 & 7.1546 & Temperate & $\mathrm{DBF}$ & 71 \\
\hline IT-Non & 44.6898 & 11.0887 & Temperate & $\mathrm{MF}$ & 10 \\
\hline IT-PT1 & 45.2009 & 9.0610 & Temperate & $\mathrm{DBF}$ & 13 \\
\hline IT-Ren & 46.5869 & 11.4337 & Boreal & ENF & 188 \\
\hline IT-Ro1 & 42.4081 & 11.9300 & Temperate & DBF & 10 \\
\hline
\end{tabular}

Continued on next page 
Table 3.A1 - Continued from previous page

\begin{tabular}{|c|c|c|c|c|c|}
\hline Site ID & Lat $\left[{ }^{\circ} \mathrm{N}\right]$ & Long $\left[{ }^{\circ} \mathrm{E}\right]$ & Climate type & Vegetation type & Forest age \\
\hline IT-Ro2 & 42.3903 & 11.9209 & Temperate & DBF & 19 \\
\hline IT-SR2 & 43.7320 & 10.2910 & Temperate & ENF & 64 \\
\hline IT-SRo & 43.7279 & 10.2844 & Temperate & ENF & 54 \\
\hline IT-Vig & 45.3167 & 8.8500 & Temperate & DBF & 15 \\
\hline JP-Tak & 36.1462 & 137.4230 & Boreal & $\mathrm{DBF}$ & 72 \\
\hline JP-Tef & 45.0563 & 142.1062 & Boreal & $\mathrm{MF}$ & 121 \\
\hline JP-Tom & 42.7395 & 141.5149 & Boreal & MF & 48 \\
\hline MY-PSO & 2.9730 & 102.3062 & Tropical & $\mathrm{EBF}$ & 106 \\
\hline NL-Loo & 52.1666 & 5.7436 & Temperate & ENF & 106 \\
\hline PA-SPn & 9.3181 & -79.6346 & Tropical & DBF & 7 \\
\hline PT-Esp & 38.6394 & -8.6018 & Temperate & $\mathrm{EBF}$ & 12 \\
\hline PT-Mi1 & 38.5407 & -8.0004 & Temperate & $\mathrm{EBF}$ & 88 \\
\hline RU-Fyo & 56.4615 & 32.9221 & Boreal & ENF & 236 \\
\hline RU-SkP & 62.2550 & 129.1680 & Boreal & DNF & 161 \\
\hline RU-Zot & 60.8008 & 89.3508 & Boreal & ENF & 201 \\
\hline SE-Fla & 64.1128 & 19.4569 & Boreal & ENF & 37 \\
\hline SE-Nor & 60.0865 & 17.4795 & Boreal & ENF & 105 \\
\hline SE-Sk1 & 60.1250 & 17.9181 & Boreal & ENF & 2 \\
\hline SE-Sk2 & 60.1297 & 17.8401 & Boreal & ENF & 33 \\
\hline SK-Tat & 49.1208 & 20.1635 & Boreal & ENF & 0 \\
\hline UK-Gri & 56.6072 & -3.7981 & Temperate & ENF & 21 \\
\hline UK-Ham & 51.1208 & -0.8608 & Temperate & DBF & 64 \\
\hline US-Bar & 44.0646 & -71.2881 & Boreal & DBF & 128 \\
\hline US-Blo & 38.8953 & -120.6328 & Temperate & ENF & 13 \\
\hline US-Bn1 & 63.9198 & -145.3780 & Boreal & ENF & 83 \\
\hline US-Bn2 & 63.9198 & -145.3780 & Boreal & DBF & 16 \\
\hline US-Dk2 & 35.9736 & -79.1004 & Temperate & DBF & 98 \\
\hline US-Dk3 & 35.9782 & -79.0942 & Temperate & ENF & 21 \\
\hline US-Fmf & 35.1426 & -111.7273 & Temperate & ENF & 150 \\
\hline US-Fuf & 35.0890 & -111.7620 & Temperate & ENF & 101 \\
\hline US-GBT & 41.3658 & -106.2397 & Boreal & ENF & 176 \\
\hline US-GLE & 41.3665 & -106.2399 & Boreal & ENF & 184 \\
\hline US-Ha1 & 42.5378 & -72.1715 & Boreal & DBF & 96 \\
\hline US-Ha2 & 42.5393 & -72.1779 & Boreal & ENF & 91 \\
\hline US-Ho1 & 45.2041 & -68.7402 & Boreal & ENF & 206 \\
\hline US-Ho2 & 45.2091 & -68.7470 & Boreal & ENF & 208 \\
\hline US-KS1 & 28.4583 & -80.6709 & Temperate & ENF & 7 \\
\hline US-LPH & 42.5419 & -72.1850 & Boreal & DBF & 98 \\
\hline US-Me1 & 44.5794 & -121.5000 & Temperate & ENF & 2 \\
\hline US-Me2 & 44.4523 & -121.5574 & Temperate & ENF & 94 \\
\hline US-Me3 & 44.3154 & -121.6078 & Temperate & ENF & 20 \\
\hline US-Me4 & 44.4992 & -121.6224 & Temperate & ENF & 24 \\
\hline US-Me5 & 44.4372 & -121.5668 & Temperate & ENF & 22 \\
\hline US-Me6 & 44.3233 & -121.6078 & Temperate & ENF & 22 \\
\hline US-MMS & 39.3232 & -86.4131 & Temperate & DBF & 95 \\
\hline US-MOz & 38.7441 & -92.2000 & Temperate & DBF & 78 \\
\hline US-NC2 & 35.8031 & -76.6679 & Temperate & ENF & 14 \\
\hline
\end{tabular}

Continued on next page 
Table 3.A1 - Continued from previous page

\begin{tabular}{|c|c|c|c|c|c|}
\hline Site ID & Lat $\left[{ }^{\circ} \mathrm{N}\right]$ & Long $\left[{ }^{\circ} \mathrm{E}\right]$ & Climate type & Vegetation type & Forest age \\
\hline US-NR1 & 40.0329 & -105.5464 & Boreal & ENF & 110 \\
\hline US-Oho & 41.5545 & -83.8438 & Boreal & DBF & 50 \\
\hline US-PFa & 45.9459 & -90.2723 & Boreal & $\mathrm{MF}$ & 150 \\
\hline US-Prr & 65.1237 & -147.4876 & Boreal & ENF & 98 \\
\hline US-SP1 & 29.7381 & -82.2188 & Temperate & ENF & 63 \\
\hline US-SP2 & 29.7648 & -82.2448 & Temperate & ENF & 4 \\
\hline US-SP3 & 29.7548 & -82.1633 & Temperate & ENF & 12 \\
\hline US-SP4 & 29.8028 & -82.2031 & Temperate & ENF & 0 \\
\hline US-Syv & 46.2420 & -89.3477 & Boreal & MF & $>150$ \\
\hline US-UMB & 45.5598 & -84.7138 & Boreal & DBF & 93 \\
\hline US-UMd & 45.5625 & -84.6975 & Boreal & DBF & 90 \\
\hline US-WBW & 35.9588 & -84.2874 & Temperate & DBF & 110 \\
\hline US-WCr & 45.8059 & -90.0799 & Boreal & DBF & 96 \\
\hline US-Wi0 & 46.6188 & -91.0814 & Boreal & ENF & 7 \\
\hline US-Wi1 & 46.7305 & -91.2329 & Boreal & DBF & 15 \\
\hline US-Wi3 & 46.6347 & -91.0987 & Boreal & DBF & 66 \\
\hline US-Wi4 & 46.7393 & -91.1663 & Boreal & ENF & 66 \\
\hline US-Wi5 & 46.6531 & -91.0858 & Boreal & ENF & 9 \\
\hline US-Wi8 & 46.7223 & -91.2524 & Boreal & DBF & 2 \\
\hline US-Wi9 & 46.6188 & -91.0814 & Boreal & ENF & 16 \\
\hline US-Wrc & 45.8205 & -121.9520 & Temperate & ENF & $>150$ \\
\hline VU-Coc & -15.4427 & 167.1920 & Tropical & $\mathrm{EBF}$ & 20 \\
\hline
\end{tabular}

\section{Carbon losses not measured by flux towers}

Table 3.A2: Estimates of the $\mathbf{C}$ losses which are not measured by flux towers. Mean estimates \pm standard deviation are shown. Units are in $\mathrm{PgC}_{\text {year }}{ }^{-1}$.

\begin{tabular}{l|llll} 
& $\mathrm{E}_{\text {harvest }}$ & $\mathrm{E}_{\text {fire }}$ & $\mathrm{E}_{\text {reduced }}$ & $\mathrm{E}_{\text {river outgas }}$ \\
\hline Boreal & $-0.29 \pm 0.010$ & $-0.15 \pm 0.075$ & -0.020 & $-0.030 \pm 0.030$ \\
Temperate & $-0.24 \pm 0.020$ & $-0.033 \pm 0.017$ & -0.040 & $-0.080 \pm 0.020$ \\
Tropics & $-0.32 \pm 0.02$ & $-0.32 \pm 0.16$ & -0.34 & $-0.42 \pm 0.090$
\end{tabular}


Eddy-covariance sites

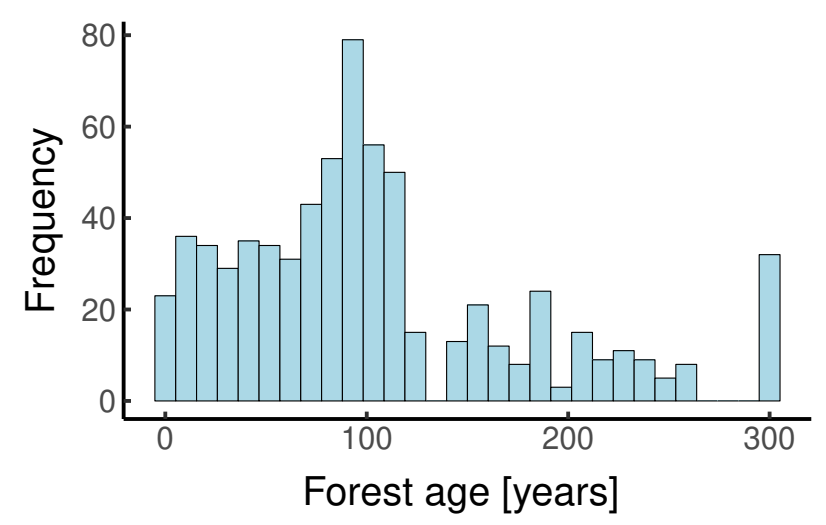

Figure 3.A1: Histogram of forest age from young to old-growth forests in the dataset used in this study.

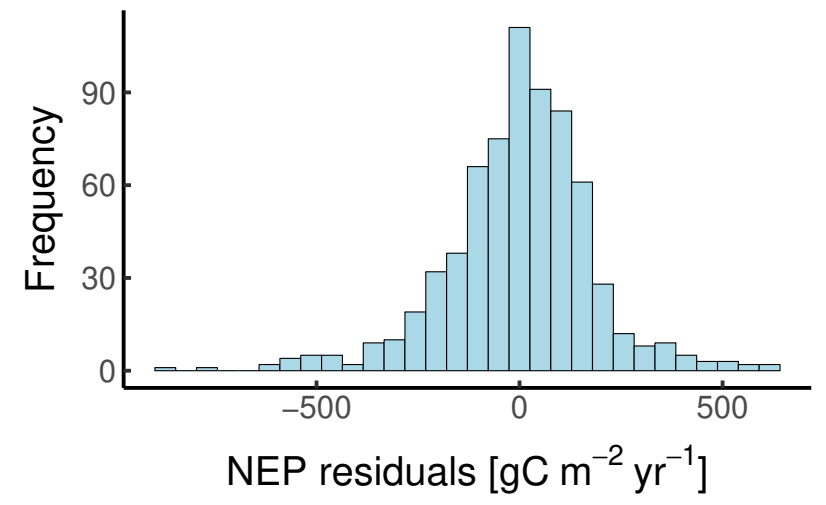

Figure 3.A2: Histogram of the NEP model residuals for the AC-Amiro approach. 


\section{Forest age map assessment}

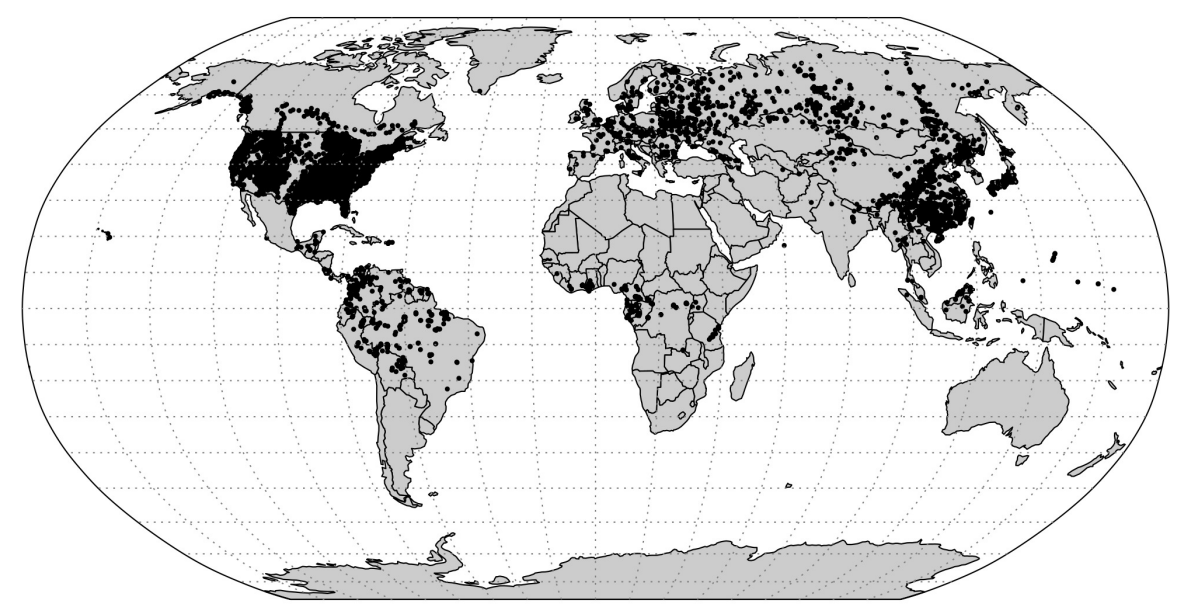

Figure 3.A3: Global distribution of the different forest inventory plots used for the forest age maps.

(a)

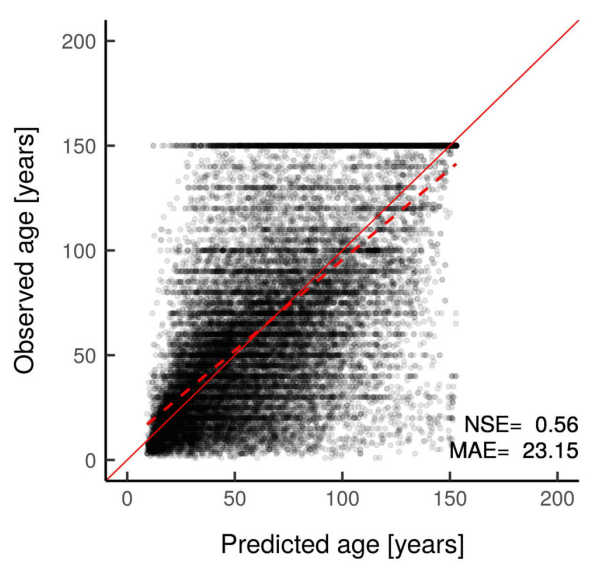

(b)
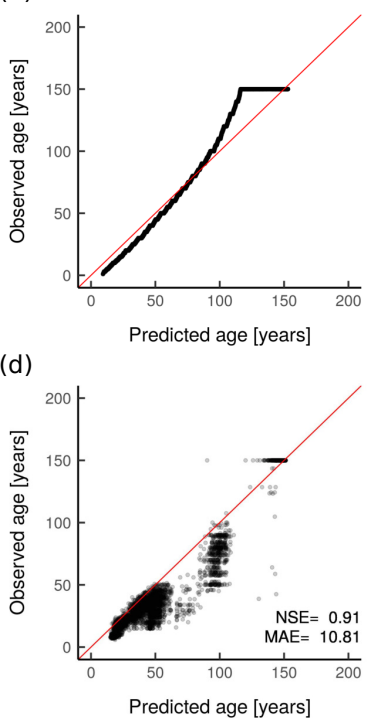

(c)

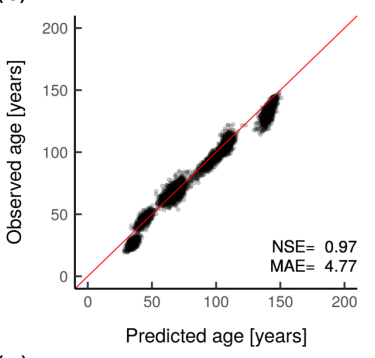

(e)

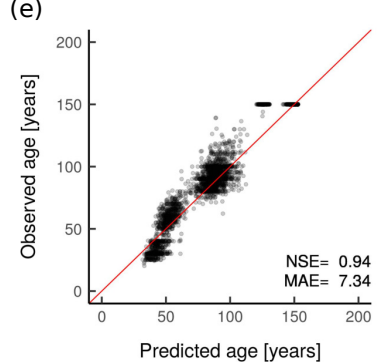

Figure 3.A4: Cross-validated comparison of predicted vs. observed forest age estimates (a). Qqplot (b) and the model performance per $10^{\circ}$ latitudinal bins computed on the mean (c), quantile $25 \%$ (d), and quantile $75 \%$ (e) are also shown. 


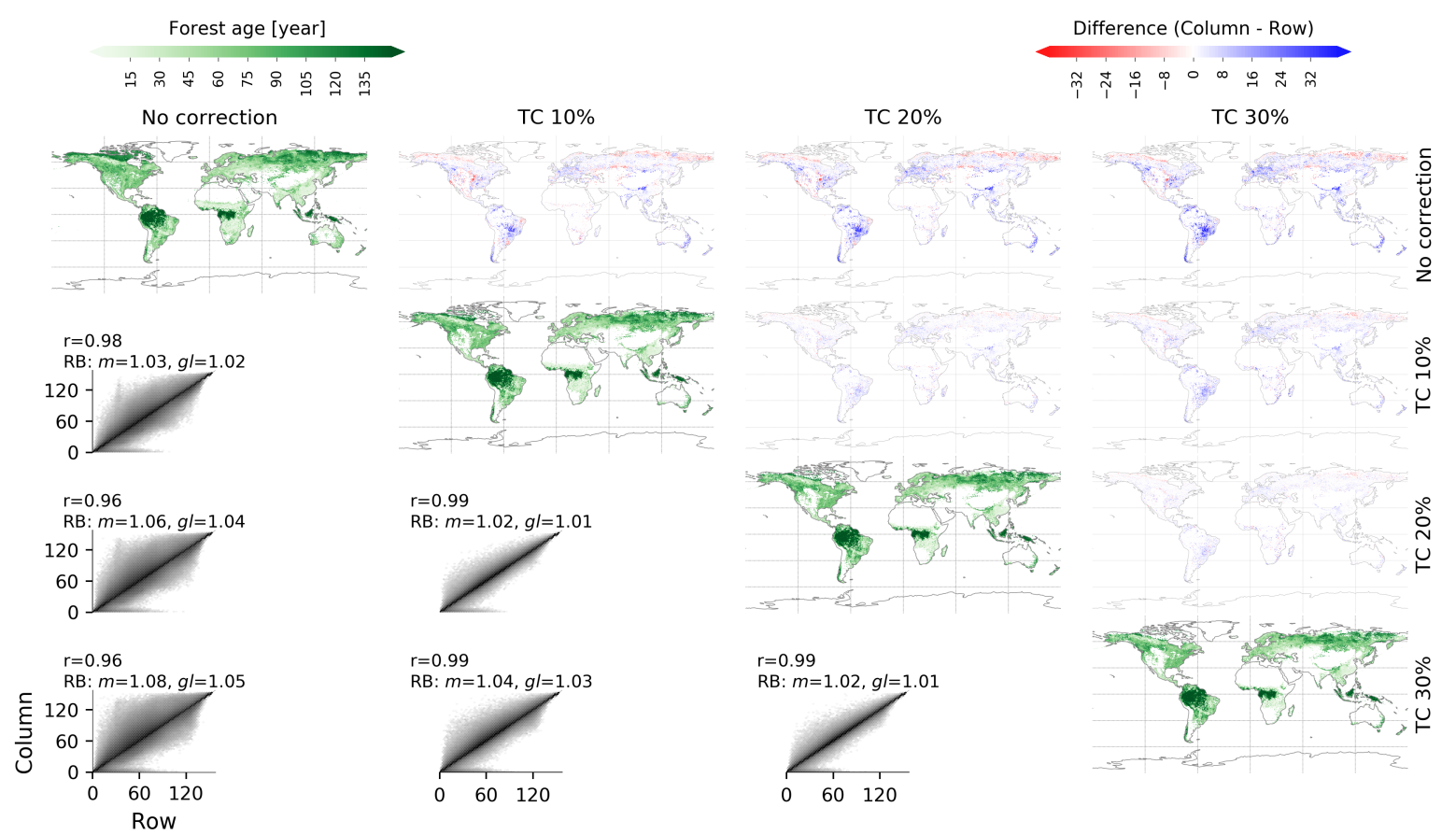

Figure 3.A5: Sensitivity of the presented age product (MPI-age) to different tree cover thresholds: no correction, $10 \%, 20 \%$, and $30 \%$ tree cover correction. 


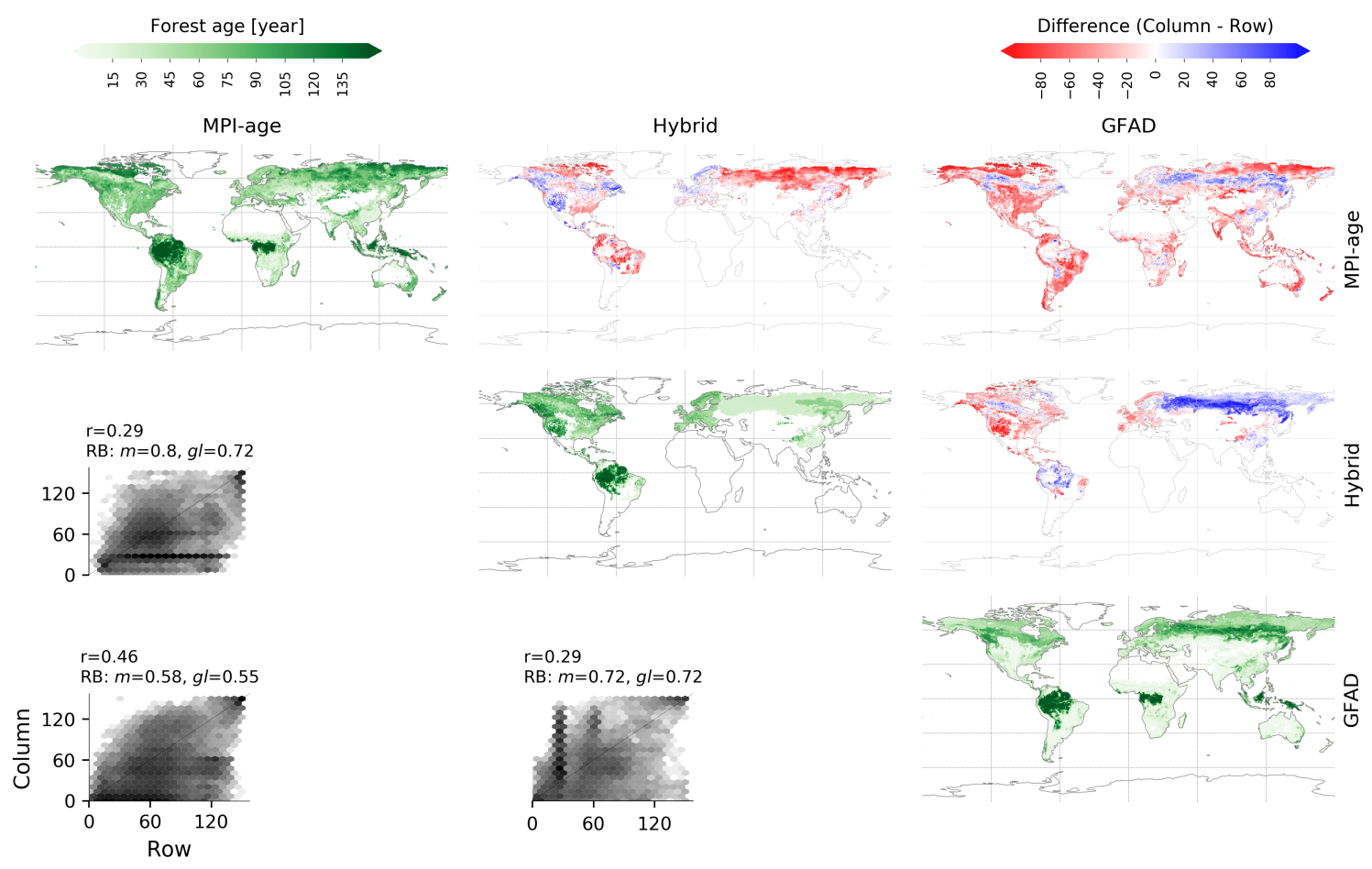

Figure 3.A6: Comparison of the presented age product (MPI-age) with a tree cover correction of $15 \%$ against regional age products (Chazdon et al., 2016; Loboda and Chen, 2017; Moreno et al., 2017; Pan et al., 2011a; Zhang et al., 2017) (Hybrid) and the GFAD forest age map (Poulter et al., 2019) (GFAD). 


\section{Uncertainty estimates of global forest NEP}

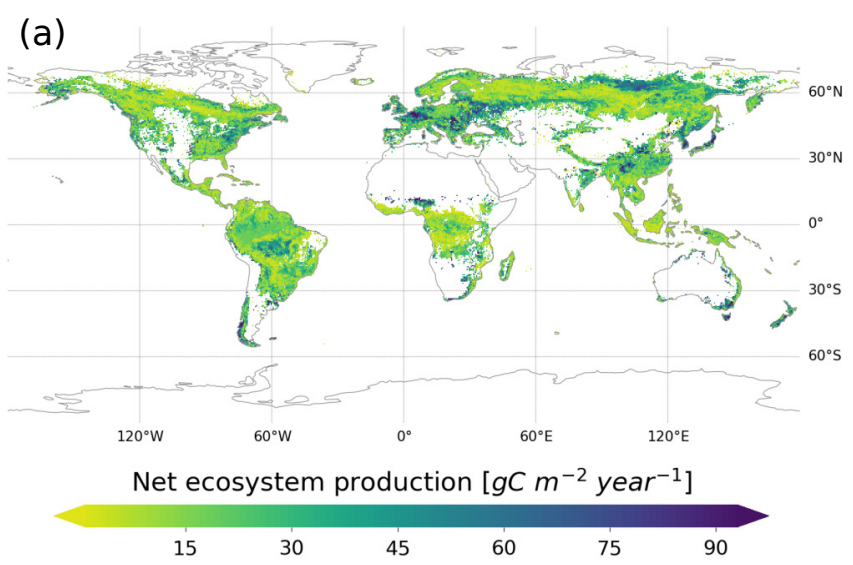

(b)

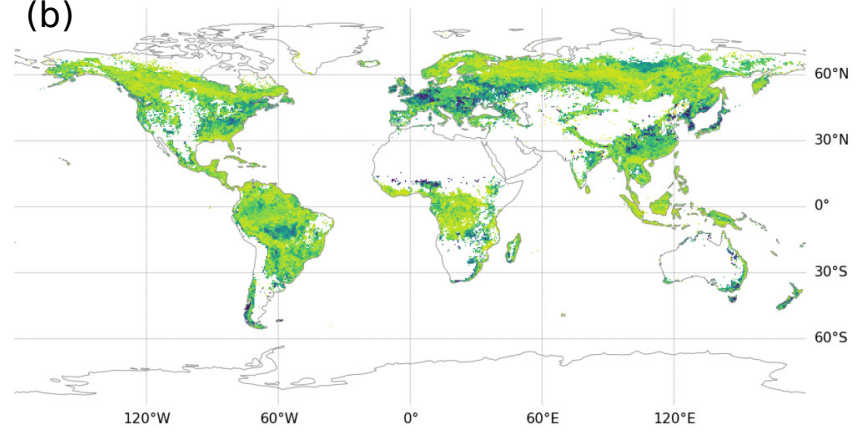

Net ecosystem production [ $\mathrm{gC} \mathrm{m}^{-2}$ year $^{-1}$ ]

$\begin{array}{lllll}15 & 30 & 45 & 60 & 75\end{array}$

(c)

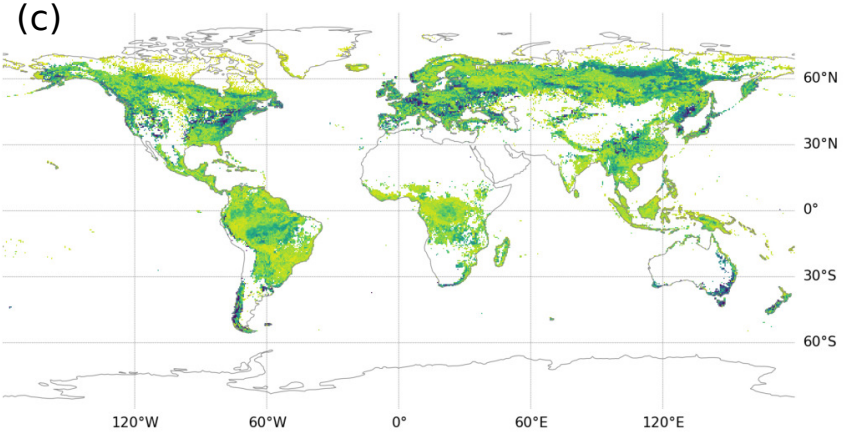

Net ecosystem production $\left[\mathrm{gC} \mathrm{m}^{-2}\right.$ year $\left.^{-1}\right]$

25

75

$100 \quad 125$

Figure 3.A7: Spatial patterns of the global NEP estimates's uncertainties for the three approaches. Uncertainties were computed by calculating the inter-quantile range of the $45 \mathrm{NEP}$ members produced. 


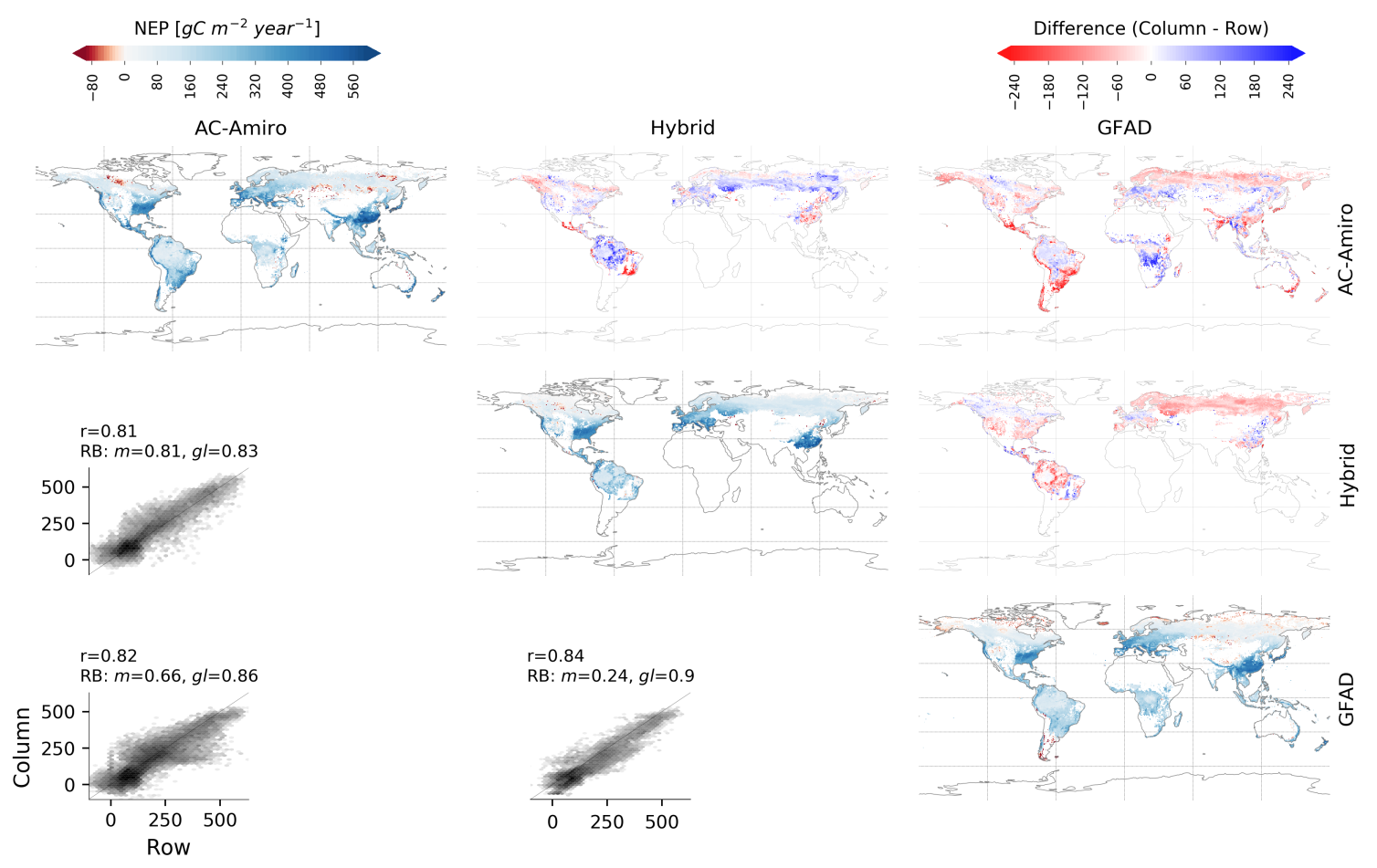

Figure 3.A8: Comparison of global NEP estimates using the presented age product (AC-Amiro) against a combination of published forest age regional maps (Chazdon et al., 2016; Loboda and Chen, 2017; Moreno et al., 2017; Pan et al., 2011a; Zhang et al., 2017) (Hybrid) and the GFAD (Poulter et al., 2019) (GFAD using the AC-Amiro model). 


\section{Chapter 4}

\section{Memory effects of climate and vegetation affecting net ecosystem $\mathrm{CO}_{2}$ fluxes in global forests}

This chapter is based on:

S. Besnard, N. Carvalhais, M. A. Arain, A. Black, B. Brede, N. Buchmann, J. Chen, J. G. P. W. Clevers, L. P. Dutrieux, F. Gans, M. Herold, M. Jung, Y. Kosugi, A. Knohl, B. E. Law, E. Paul-Limoges, A. Lohila, L. Merbold, O. Roupsard, R. Valentini, S. Wolf, X. Zhang, and M. Reichstein (2019a). "Memory effects of climate and vegetation affecting net ecosystem $\mathrm{CO}_{2}$ fluxes in global forests". PLOS ONE 14.2, e0211510 


\subsection{Abstract}

Forests play a crucial role in the global carbon (C) cycle by storing and sequestering a substantial amount of $\mathrm{C}$ in the terrestrial biosphere. Due to temporal dynamics in climate and vegetation activity, there are significant regional variations in carbon dioxide $\left(\mathrm{CO}_{2}\right)$ fluxes between the biosphere and atmosphere in forests that are affecting the global $\mathrm{C}$ cycle. Current forest $\mathrm{CO}_{2}$ flux dynamics are controlled by instantaneous climate, soil, and vegetation conditions, which carry legacy effects from disturbances and extreme climate events. Our level of understanding from the legacies of these processes on net $\mathrm{CO}_{2}$ fluxes is still limited due to their complexities and their long-term effects. Here, we combined remote sensing, climate, and eddy-covariance (EC) flux data to study net ecosystem exchange (NEE) at 185 forest sites globally. Instead of commonly used non-dynamic statistical methods, we employed a type of recurrent neural network (RNN), called Long-Short-Term Memory (LSTM) network that captures information from the vegetation and climate's temporal dynamics. The resulting data-driven model integrates interannual and seasonal variations of climate and vegetation by using Landsat and climate data at each site. The presented LSTM algorithm was able to effectively describe the overall seasonal variability (modeling efficiency $=0.66$ ) and across-site (modeling efficiency $=0.42$ ) variations in NEE, while it had less success in predicting specific seasonal and interannual anomalies (modeling efficiency $=0.07$ ). This analysis demonstrated that an LSTM approach with embedded climate and vegetation memory effects outperformed a non-dynamic statistical model (i.e. Random Forest) for estimating NEE. Additionally, it is shown that the vegetation mean seasonal cycle embeds most of the information content to realistically explain the spatial and seasonal variations in NEE. These findings show the relevance of capturing memory effects from both climate and vegetation in quantifying spatio-temporal variations in forest NEE. 


\subsection{Introduction}

Forests cover about $30 \%$ of the terrestrial surface of our planet, accounting for $75 \%$ of gross primary productivity (GPP), and store 45\% of all terrestrial carbon (C) (Beer et al., 2010; Gower, 2003; Pan et al., 2011b). This fundamental role highlights the importance of understanding forest $\mathrm{C}$ dynamics, which are generally driven by climatic conditions and vegetation dynamics as well as natural and anthropogenic disturbances (Le Quéré et al., 2018; Reichstein et al., 2013; Zhu et al., 2016). Changes in climate and disturbance regime can influence the development, structure, and functioning of forest ecosystems (Birdsey et al., 2006; Johnson and Curtis, 2001; Liu et al., 2011; Schimel, 2007; Williams et al., 2012; Woodbury et al., 2007), therefore causing anomalies in the net ecosystem exchange (NEE). As a result, quantifying the effects of climatic variations and forest disturbances on biosphere-atmosphere carbon dioxide $\left(\mathrm{CO}_{2}\right)$ fluxes across-scales has considerable importance for understanding the net $\mathrm{C}$ balance of forest ecosystems (Amiro et al., 2010; Carvalhais et al., 2010; Thornton et al., 2002; Zscheischler et al., 2017).

Disturbances, such as fire, disease, insect outbreaks, drought, windthrow, or harvesting, can shift forest ecosystems into early stages of ecological succession (Franklin et al., 2002; Odum, 1969). These events can potentially trigger an accelerated release of stored $C$ back to the atmosphere by reducing the amount of photosynthetic tissue and also by increasing the pool of respiring detritus material for subsequent gradual release (Amiro et al., 2010; Bowman et al., 2009; Ciais et al., 2014a; Moore et al., 2013). During recovery, forests accumulate biomass and potentially sequester $\mathrm{C}$ from the atmosphere at rates that could alter current trends of atmospheric C cycling (Schimel, 2007). Post-disturbance successional trajectories are often complex, depending on pre-disturbance forest structure and function, disturbance type, frequency, and intensity (Gough et al., 2007; Meigs et al., 2009) as well as the climate of the region (Anderson-Teixeira et al., 2013; Chazdon et al., 2016) and land management. Some disturbances, such as insect outbreaks, can slow down recovery process during regeneration or transform forests from closed to open canopies (Donato et al., 2013), while other low to moderate severity disturbances increase structural complexity leading to less of an impact on mid-succession net primary productivity (NPP) than is often assumed (Gough et al., 2016). Therefore, climate and disturbance regimes contribute to interannual and seasonal variations in forest net $\mathrm{CO}_{2}$ fluxes. Changes in climate may also exacerbate the frequency and intensity of extreme meteorological events (e.g. droughts (Reichstein et al., 2013) or associated fire events (Seidl et al., 2011; Turner, 2010)), thereby increasing both mortality rates and the vulnerability of forest ecosystems (Seidl et al., 2014), which would necessarily impact the dynamics of ecosystem C cycle.

Current response patterns observed in forest $\mathrm{CO}_{2}$ fluxes depend on the contemporaneous environment conditions as well as on the so-called memory effects of disturbances, climatic variation, and their interactions (Monger et al., 2015; Seidl et al., 2014). In fact, disturbances or climate extreme events exert both instantaneous and lagged impacts on biosphere-atmosphere $\mathrm{C}$ fluxes (Frank et al., 2015; Reichstein et al., 2013). Memory effects can be defined as the influence that 
past events have on the present or future responses of an ecosystem to environmental conditions. Extensive research has been done to understand climate and disturbance memory effects on $\mathrm{CO}_{2}$ fluxes (i.e. NEE, GPP, and ecosystem respiration (ER)) (Aubinet et al., 2018; Desai, 2014; Molen et al., 2011; Shen et al., 2016; Zhang et al., 2015b; Zielis et al., 2014). However, given the complexity of NEE responses to disturbances and climate extremes and highly non-linear processes, the legacies of these events on $\mathrm{CO}_{2}$ fluxes remain unclear (Frank et al., 2015; Vicca et al., 2014), and thus they are rarely implemented in current $\mathrm{C}$ cycle models. As such, statistical models capable of dynamically incorporating temporal information related to episodic disturbances and climatic fluctuations are required to enhance our understanding and predictive capabilities of the global C budget (Bodesheim et al., 2018; Liu et al., 2011). Recently, deep learning (DL) techniques, such as recurrent neural network (RNN), have shown the potential to capture long-term temporal dependencies and variable-length observations (Bahdanau et al., 2014; Hinton et al., 2012; Sutskever et al., 2014). Yet, DL is early in its application for $\mathrm{CO}_{2}$ flux predictions (Reichstein et al., 2018); questions related to the potential of extracting temporal information for estimating $\mathrm{CO}_{2}$ fluxes across-scales have yet to be investigated.

In this study, we explore the potential of a dynamic statistical approach - a type of RNN, called Long-Short-Term Memory (LSTM) model - to characterize the memory effects of disturbance and climate variations on NEE across temporal and spatial scales at 185 forest and woodland FLUXNET sites globally utilizing remote sensing, climate, and eddy-covariance (EC) datasets. In particular, this study focuses on: (1) comparing the statistical power of an LSTM approach to a Random Forest (RF) algorithm in predicting ecosystem level NEE, and (2) assessing the importance of capturing the memory effects of vegetation and climate to predict forest NEE using data-driven LSTMs. The analysis focuses on the variations in NEE spatially and temporally for seasonal, monthly, and interannual anomalies, for which a factorial experiment was designed as explained below. We propose that the application of dynamic statistical approaches results in estimating net $\mathrm{CO}_{2}$ fluxes across-scales more realistically by including the responses of NEE to antecedent climate and disturbance conditions.

\subsection{Material and methods}

\subsubsection{Eddy-covariance data and quality check}

The current dataset consists of 185 forest and woodland sites (Table 4.A1) composed of five plant functional type (PFT): deciduous broadleaf forest (DBF) $(\mathrm{N}=42)$, deciduous needleleaf forest $(\mathrm{DNF})(\mathrm{N}=1)$, evergreen broadleaf forest $(\mathrm{EBF})(\mathrm{N}=27)$, evergreen needleleaf forest $(\mathrm{ENF})$ $(\mathrm{N}=81)$, mixed forest (MF) $(\mathrm{N}=14)$, woody savanna (WSA) $(\mathrm{N}=10)$, and savanna (SAV) $(\mathrm{N}=10)$; and four climate class: arid $(\mathrm{N}=11)$, boreal $(\mathrm{N}=67)$, temperate $(\mathrm{N}=86)$, and tropical $(\mathrm{N}=21)$. We aggregated DBF and DNF into a deciduous forest class, EBF and ENF into an evergreen forest class, and SAV and WSA into a savanna class. The sites were part of both version 2 of the 
LaThuile FLUXNET and the FLUXNET2015 datasets (https: / / fluxnet.fluxdata .org) of the FLUXNET network (Baldocchi, 2008; Baldocchi et al., 2001). For each site, continuously measured or gap-filled NEE (i.e. night-time partitioning method (Reichstein et al., 2005)) and microclimatic variables (i.e. air temperature $\left(\mathrm{T}_{\text {air }}\right)$, precipitation (Precip), global radiation $(\mathrm{Rg})$, and vapor pressure deficit (VPD)) were obtained at half-hourly time intervals from the FLUXNET datasets. Half-hourly NEE and microclimatic variables were aggregated into monthly averages (i.e. seasonal cycle). Only monthly NEE observations with more than $80 \%$ of the original or good quality gap-filled data were considered in this analysis (Papale et al., 2006). A total of $\simeq 14,000$ observed or gapfilled monthly NEE flux data was used, from which $\simeq 1,500$ monthly observations were collected in forests younger than 30 years.

\subsubsection{Remote sensing data}

For each FLUXNET site, the entire multi-temporal collection 1 from the Landsat 4, 5, 7 and 8 archives (https://landsat.usgs.gov/) spanning the past 30 years at 30 meters resolution was collected. Landsat data have been pre-processed using the Landsat Ecosystem Disturbance Adaptive Processing System (LEDAPS) (Schmidt et al., 2013) and the Landsat Surface Reflectance Code (LaSRC) (https://landsat.usgs.gov/landsat-surface-reflectancedata-products) for atmospheric correction. Poor quality retrievals due to the clouds, cloud shadows, snow, and ice were masked out (Zhu et al., 2015; Zhu and Woodcock, 2012). The data extraction and the pre-processing chains (i.e. cloud, cloud shadow masking, and downloading) were implemented in the Google Earth Engine (GEE) platform (Gorelick et al., 2017) (https://earthengine.google.com/). The seven spectral bands of the Landsat product were used; i.e. blue, green, red, near-infrared (NIR), shortwave infrared (SWIR) 1, SWIR 2, and thermal infrared (TIR) (https://landsat .usgs .gov/what-are-band-designationslandsat-satellites). To better represent the EC footprint area, a circular buffer of $500 \mathrm{~m}$ radius centered on each FLUXNET tower was defined for which a mean value within the different Landsat cutouts was extracted. Note that the proposed LSTM approach can only be implemented with regular time series, but most of the Landsat time series were irregular due to cloud cover or data quality issues. A first gap-filling procedure was conducted by predicting monthly reflectance values for each Landsat band with a RF model (Breiman, 2001; Kuhn, 2008) using the monthly MODerate-resolution Imaging Spectroradiometer (MODIS) (MCD43A4 version 6) bands as predictive variables (Fig. 4.A1). The gap-filling procedure was completed for the remaining gaps in the entire Landsat time series (i.e. from the 1980s to now) by predicting each Landsat band with an RF model using climate variable (i.e. $\mathrm{T}_{\text {air }}$, Precip, Rg, VPD, and potential global radiation $\left(\mathrm{R}_{\text {pot }}\right)$ ), PFT, month of the year, and latitude as predictive variables (Fig. 4.1 and 4.A2). For the two aforementioned gap-filling procedures, the best set of the predictors for predicting each Landsat band independently was obtained with a feature selection analysis (i.e. the Boruta algorithm (Kursa and Rudnicki, 2010)). 


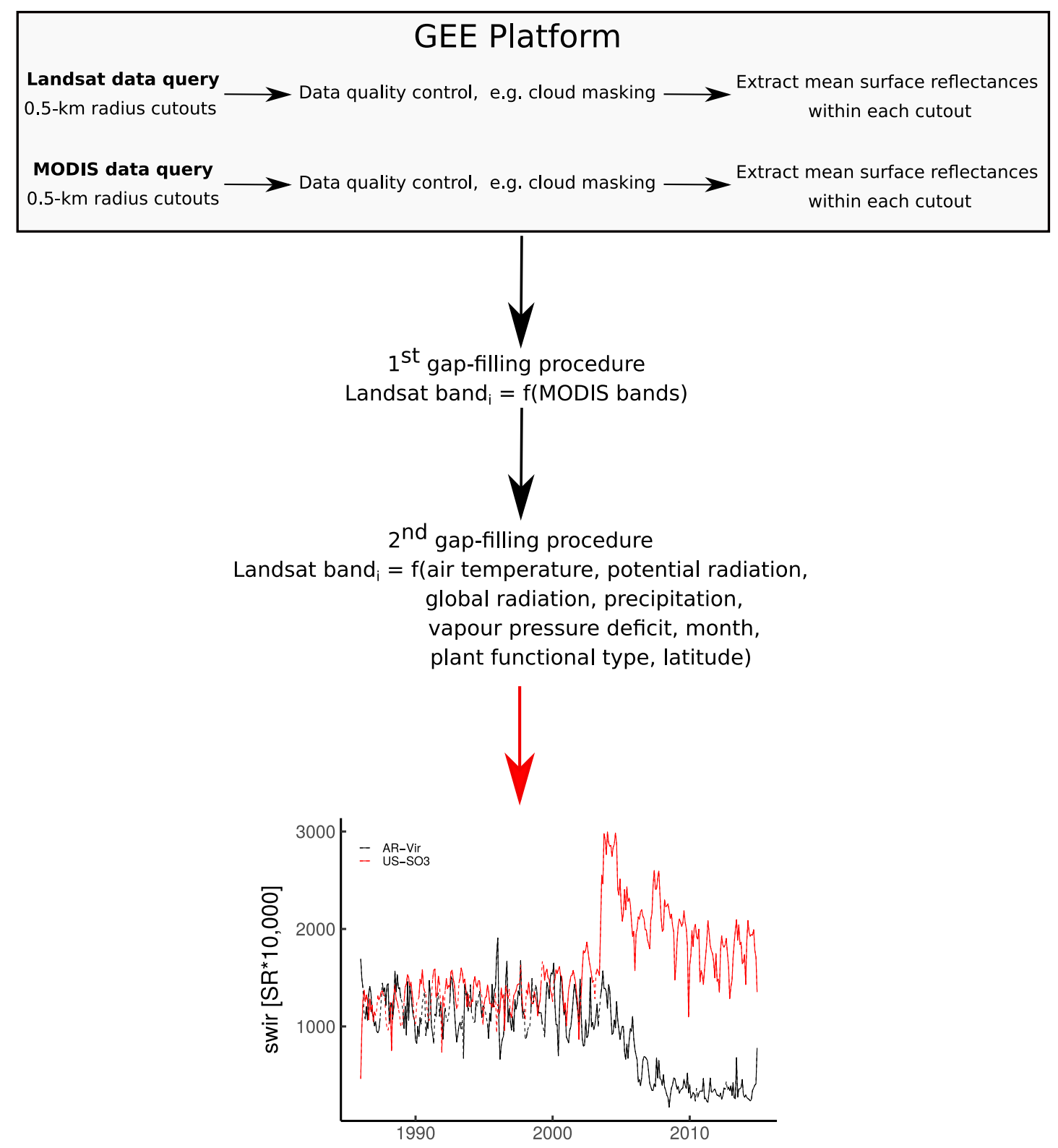

Figure 4.1: Flowchart of the Landsat data extraction and post-processing. SWIR = Shortwave Infrared. SR = Surface Reflectance. Monthly temporal gap-filled Landsat time series from 1982 to 2015 of the shortwave Infrared band are shown for AR-Vir and US-SO3 sites where, respectively, afforestation-reforestation and fire followed by a regrowth were reported in 2003 . The solid and the dashed lines depict the real observations and the gap-filled data, respectively.

\subsubsection{Climate data}

Long-term time series of $\mathrm{T}_{\text {air }}$, Precip, Rg, and VPD were down-scaled for the period of 19792015 from the ERA-Interim datasets (Dee et al., 2011). For each site, the three nearest grid cells in the ERA-Interim datasets were extracted and several statistical models were trained (i.e. relational logistic regression, kernel ridge regression, Gaussian processes regression, and 
neural networks) for each target variable (i.e. $\mathrm{T}_{\text {air }}$, Precip, $\mathrm{Rg}$, and VPD) using the time series of the three nearest gridcells as predictive variables. For each target variable and at each site, the best statistical model was consequently selected based on the highest Nash-Sutcliffe model efficiency (NSE) (Fig. 4.A3). These down-scaled climate time series were used to gap-fill climate observations measured at the site level in order to have climatic data covering the entire remote sensing data period.

\subsubsection{Recurrent neural network model for dynamic modeling}

RNNs were employed to learn vegetation and climate history based on sequential observations (https://github.com/bgi-jena/RNNFluxes.jl.git) (Reichstein et al., 2018). RNNs are effective tools for capturing temporal information from sequential data. We used a special kind of RNN, capable of learning long-term dependencies, called LSTM networks (Hochreiter and Schmidhuber, 1997). LSTMs utilize relevant information from all previous observations and are suitable to model long-term temporal dependencies.

Monthly climate data (i.e. $\mathrm{T}_{\text {air }}$, Precip, Rg, and VPD) and Landsat raw bands from the period of 1982 to 2015 were used to train the LSTM models (Fig. 4.2). A single layered LSTM was used to learn information based on the input of the current and of all previous observations. At each training iteration, a loss function (i.e. mean squared error (MSE)) was calculated by comparing monthly predicted and observed NEE. The loss function was used to derive the gradients for backpropagation over the entire sequence (Rumelhart et al., 1986). The gradients were further used by an Adam optimizer (Kingma and Ba, 2014) for adjusting the weights of the connections in the model so as to minimize the loss function. During the learning procedure, an evaluation set (i.e. $20 \%$ of the training set) was created. The learning procedure was stopped when the calculated loss function on the evaluation set did not decrease after 500 iterations (i.e. early stopping). Additionally, there was a grid search of the LSTM's hyperparameters; i.e. learning rate $(0.1$ or 0.01$)$, number of hidden neurons $(10,20$, or 30$)$, and dropout ( 0 or 0.5$)$ (Srivastava et al., 2014) to select the optimal set of hyperparameters. Due to the random initialization of the LSTMs, 50 runs for each model set-up were performed to assess the uncertainty of the model outputs.

\subsubsection{Experimental design}

In order to understand vegetation and climate memory effects on NEE, a trained LSTM with monthly climatic data (i.e. $\mathrm{T}_{\text {air }}$, Precip, Rg, and VPD) and monthly Landsat data (i.e. blue, green, red, NIR, SWIR 1, SWIR 2, and TIR bands) was benchmarked against a series of different model set-ups (Table 4.1 and Fig. 4.3). A comparison of the following five distinct experimental set-ups was performed: (1) LSTM model using the full depth of the Landsat time series and climate data (hereafter LSTM); (2) LSTM model where the orders of the predictor-target pairs were randomly permuted so that the instantaneous link between dependent and independent variables were kept 


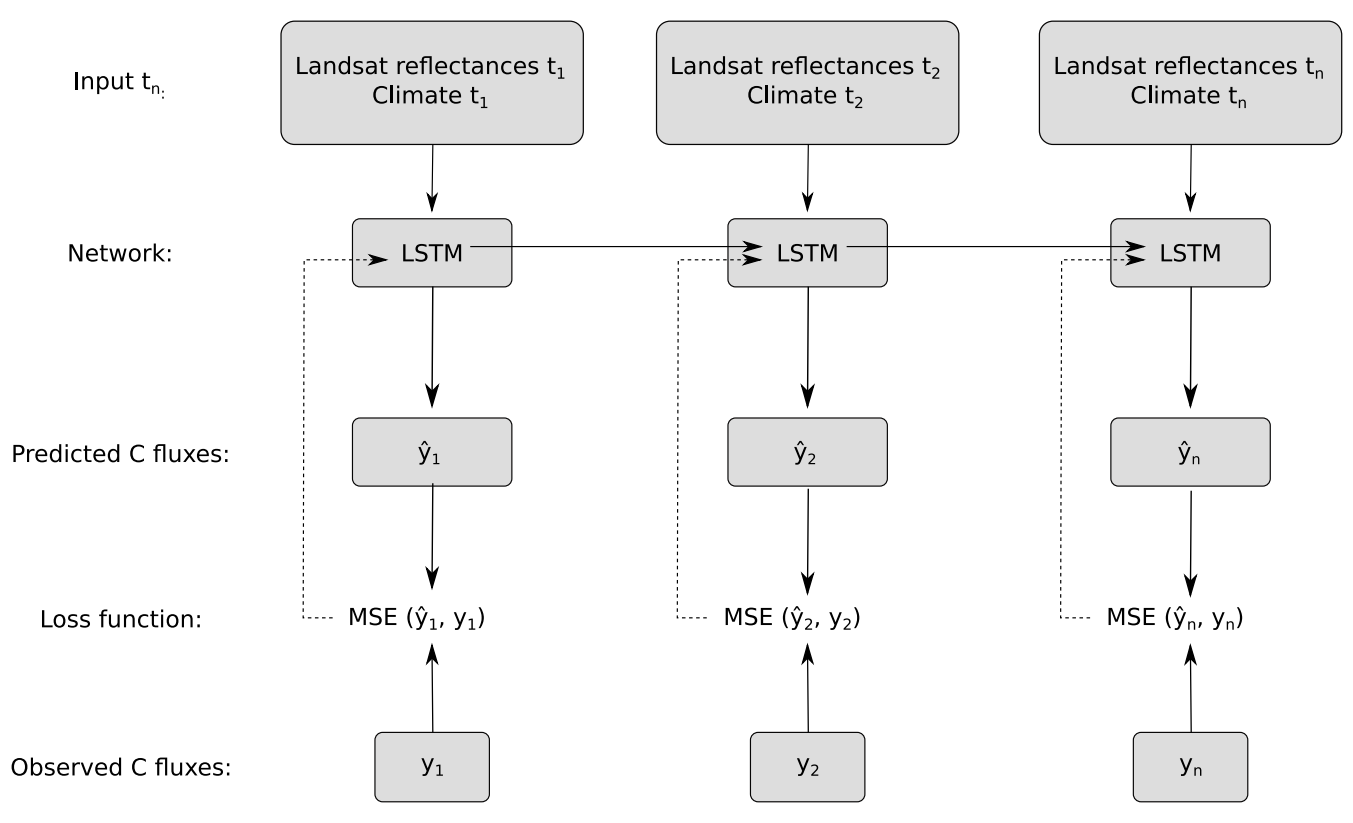

Figure 4.2: Flowchart of the proposed LSTM approach. Figure adapted from (Rußwurm and Körner, 2017). Each individual timestep is a monthly observation for the period 1982 to 2015. Landsat surface reflectances correspond to the seven spectral bands of the Landsat product; i.e. blue, green, red, near infrared, shortwave infrared 1, shortwave infrared 2, and thermal infrared. Climate corresponds to $\mathrm{T}_{\text {air }}$, Precip, Rg, and VPD. At each time step, an LSTM layer containing a set of cells or hidden neurons $(10,20$, or 30$)$ processes information based on the input of the current and of all previous observations. Predictions of NEE were performed at each monthly timestep by using information from both current and previous observations. The loss function was only calculated when NEE observations were available; i.e. measurement periods of LaThuile and FLUXNET2015 datasets.

but the realistic temporal sequences were destroyed (hereafter $L S T M_{\text {perm }}$ ); (3) LSTM model where the Landsat time series for each band were replaced by their mean seasonal cycle (i.e. mean of each month over the Landsat time series period) while using the actual values of $\mathrm{T}_{\text {air }}$, Precip, Rg, and VPD (hereafter $L S T M_{m s c}$ ); (4) LSTM model where the Landsat time series for each band were replaced by their annual mean (i.e. mean of the monthly observations within each year over the Landsat time series period) while using the actual values of $\mathrm{T}_{\text {air }}$, Precip, $\mathrm{Rg}$, and VPD (hereafter LSTM annual), and (5) a RF model (Breiman, 2001; Kuhn, 2008) using the actual values of the Landsat time series and climate data (hereafter $R F$ ). 
Table 4.1: Design of the factorial experiment. $X$ means that the variant was used to study the respective topic of each row. $L S T M=$ LSTM model using the full depth of the Landsat time series and climate data; $L S T M_{\text {perm }}=L S T M$ model but the temporal patterns of both the predictive and the target variables were randomly permuted while instantaneous relationships between predictive and target variables were kept; $L S T M_{m s c}=L S T M$ model but the Landsat time series for each band were replaced by their mean seasonal cycle, while using the actual values of $\mathrm{T}_{\text {air }}$, Precip, Rg, and VPD; LSTM annual $=$ LSTM model but the Landsat time series for each band were replaced by their annual mean, while using the actual values of $\mathrm{T}_{\text {air }}$, Precip, $\mathrm{Rg}$, and VPD; RF $=$ Random Forest model using the actual values of the Landsat time series and climate data.

\begin{tabular}{|c|c|c|c|c|c|}
\hline & LSTM & $\mathrm{LSTM}_{\text {perm }}$ & $\mathrm{LSTM}_{m s c}$ & $\mathrm{LSTM}_{\text {anпиаl }}$ & RF \\
\hline Temporal feature extraction/Memory effects & $\mathbf{X}$ & $\mathbf{X}$ & & & $\mathbf{X}$ \\
\hline Vegetation interannual seasonal variation & $\mathbf{X}$ & & $\mathbf{X}$ & & \\
\hline Vegetation interannual variability & $\mathbf{X}$ & & & $\mathbf{X}$ & \\
\hline Comparision to non-dynamic method & $\mathbf{X}$ & & & & $\mathbf{X}$ \\
\hline
\end{tabular}

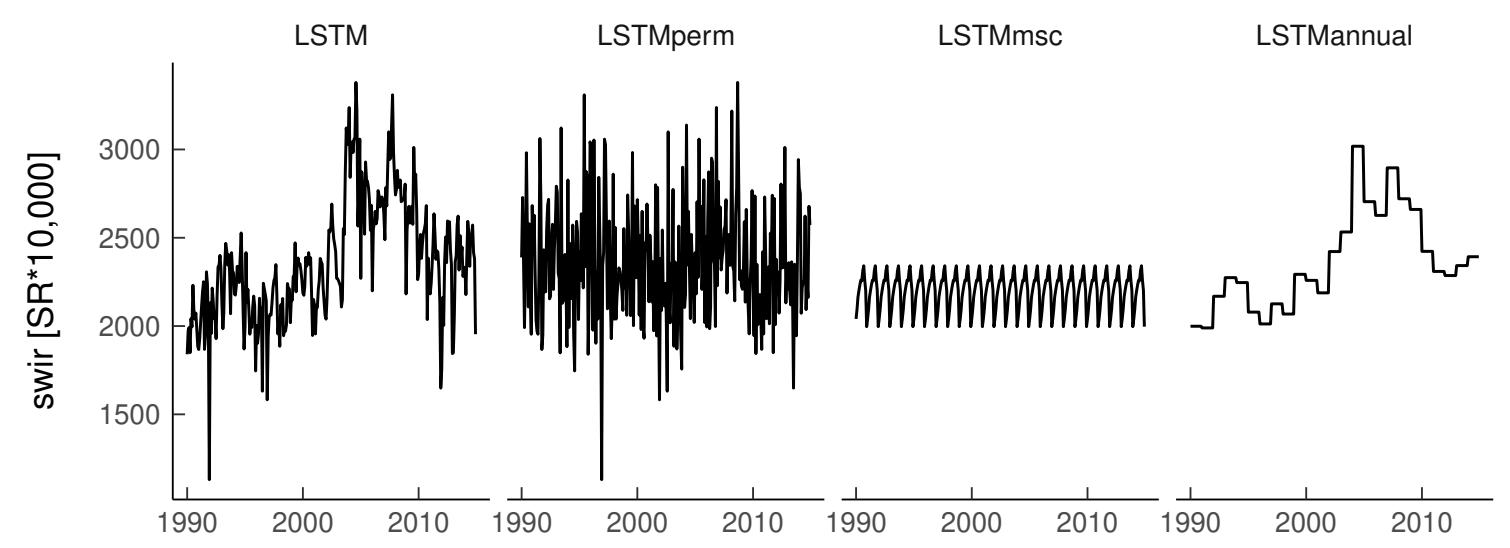

Figure 4.3: Illustration of the different Landsat time series temporal architectures of the different LSTM model set-ups for the SWIR band only for the period 1990-2015. SWIR = Short Wave Infrared. SR = Surface Reflectance. The US-SO3 site where fire followed by regrowth was reported in 2003 is shown.

Comparing the $L S T M$ with the $L S T M_{m s c}$ model set-ups served to assess the importance of including and extracting information on interannual seasonal variation of vegetation to calculate NEE for each forest site across the globe. Contrasting the LSTM annual with the LSTM reflects lost in model fitness by not including the information contained in the monthly mean seasonal cycle of vegetation. The differences between the results from the LSTM and the $L_{S T M}$ perm as well as between the LSTM and the $R F$ aimed to test the effects of extracting realistic temporal dependencies in the observations for predicting net $\mathrm{CO}_{2}$ fluxes. The $R F$ set-up also provided a comparison to commonly used data-driven statistical modeling approaches for NEE estimates 
(Jung et al., 2011; Tramontana et al., 2016) (Table 4.1). The predictive variables used in the different model set-ups are listed in table 4.2.

Table 4.2: List of predictors used in the different model set-ups. $L S T M=$ LSTM model using the full depth of the Landsat time series and climate data; $L_{S T M}$ perm $=$ LSTM model but the temporal patterns of both the predictive and the target variables were randomly permuted while instantaneous relationships between predictive and target variables were kept; $L S T M_{m s c}=L S T M$ model but the Landsat time series for each band were replaced by their mean seasonal cycle, while using the actual values of $\mathrm{T}_{\text {air }}$, Precip, Rg, and VPD; LSTM $_{\text {annual }}=$ LSTM model but the Landsat time series for each band were replaced by their annual mean, while using the actual values of $\mathrm{T}_{\text {air }}$, Precip, Rg, and VPD; RF = Random Forest model using the actual values of the Landsat time series and climate data.

\begin{tabular}{l|l} 
Model set-up & Predictors \\
\hline LSTM & 7 Landsat bands + $\mathrm{T}_{\text {air }}+$ Precip + Rg + VPD \\
LSTM $_{\text {perm }}$ & permuted [7 Landsat bands + Tair + Precip + Rg + VPD] \\
LSTM $_{\text {msc }}$ & 7 MSC Landsat bands $+\mathrm{T}_{\text {air }}+$ Precip + Rg + VPD \\
LSTM $_{\text {annual }}$ & 7 annual mean Landsat bands + Tair + Precip + Rg + VPD \\
RF & actual values of 7 Landsat bands + Tair + Precip + Rg + VPD
\end{tabular}

\subsubsection{Model training and evaluation}

The performance of each model set-up was evaluated by directly comparing model estimates with observed values of NEE for each site. These statistical models were evaluated using a 10-fold cross-validation strategy in which entire sites were assigned to each fold (Jung et al., 2011). The training of each model set-up was done using data from $\mathrm{n}_{\text {fold }}-1$, while predictions were made for the remaining fold, ensuring that the validation data were independent of the training data. The statistics used to assess the capability of the statistical models to estimate NEE were the coefficient of determination $\left(\mathrm{R}^{2}\right)$, the NSE, the root mean square error (RMSE), and mean absolute error (MAE) (Omlin and Reichert, 1999).

The predictive capacity of the different algorithms was assessed for the seasonal cycle, the seasonal anomalies, the interannual anomalies, and the across-site variability. The seasonal anomalies were computed as the difference between the monthly NEE estimates of a considered month and those of the same month averaged over the observation period for each site. The interannual anomalies were computed as the difference between the annual NEE estimates of a considered year and the annual averaged over the entire observation period for each site. Both seasonal and interannual anomalies were calculated only for the sites with at least three years of complete observations after data quality check. Across scales, the statistical models were trained using monthly time-series and the estimates were further aggregated to the corresponding scales, i.e. seasonal cycle, seasonal and interannual anomalies, and across-site.

Results were analyzed on the global dataset as well as according to PFT, bioclimatic, and forest age classes. PFT and climate classifications were found in the ancillary data files provided by 
the La Thuile or the FLUXNET2015 datasets (https://fluxnet.fluxdata.org). Forest age data were derived from a published dataset (Besnard et al., 2018). Forest age estimates were aggregated into six classes: $0-10$ years ( $\mathrm{N}=7$ sites), $10-20$ years $(\mathrm{N}=8$ sites), $20-50$ years $(\mathrm{N}=14$ sites), 50-100 ( $\mathrm{N}=27$ sites), 100-150 ( $\mathrm{N}=14$ sites), and 150- $\geq 300$ years $(\mathrm{N}=15$ sites).

\subsection{Results and Discussion}

\subsubsection{Performance of the Recurrent Neural Networks}

The proposed approach was generally able to capture the seasonal cycle well for LSTM set-ups $(\mathrm{NSE}=0.66)$, but had moderate to poor predictive capacity to explain across-site variability $(\mathrm{NSE}=0.42)$, monthly anomalies $(\mathrm{NSE}=0.07)$ or interannual anomalies $(\mathrm{NSE}=0.07)$ (Fig. 4.4). However, the proposed approach achieved comparable predictive capacity than the most recent NEE estimates based on FLUXNET data across scales (Jung et al., 2011; Tramontana et al., 2016).

(a) seasonal cycle

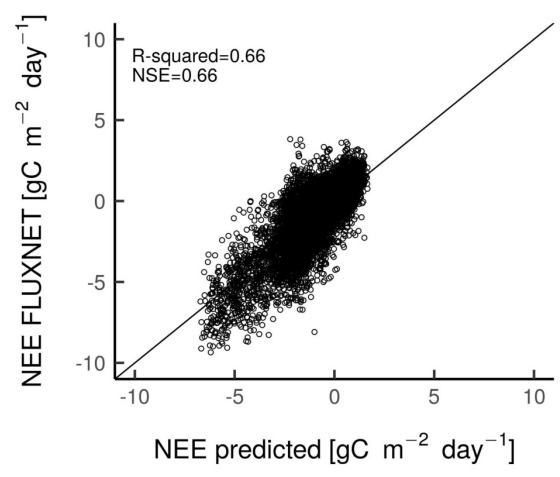

(c) across-sites

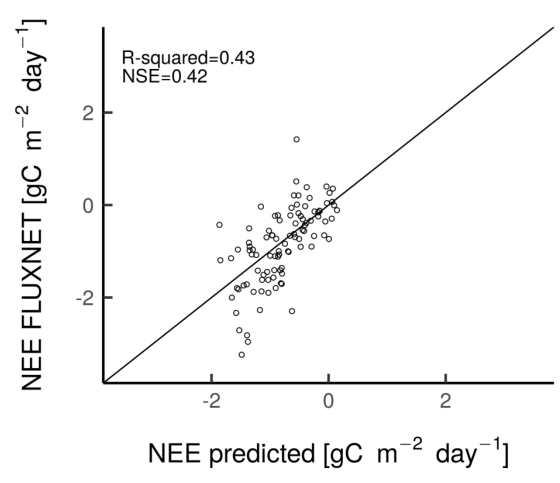

(b) seasonal anomalies

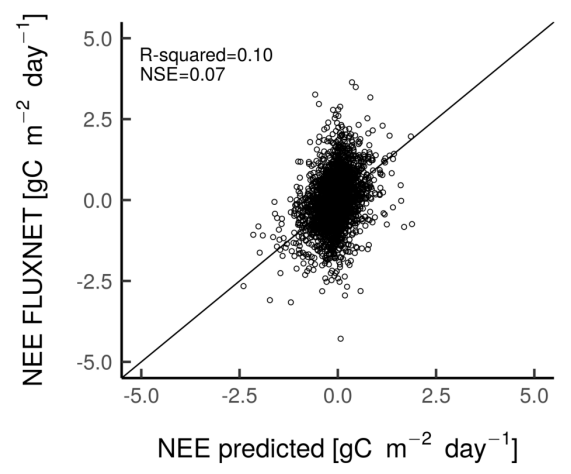

(d) interannual anomalies

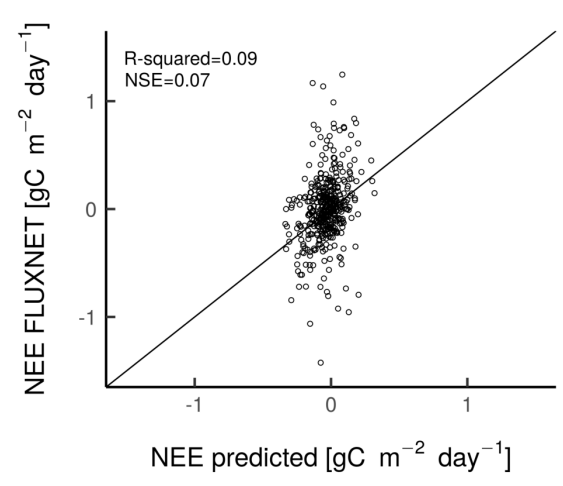

Figure 4.4: Scatterplots of observed data and the LSTM modeled NEE for the seasonal cycle (a), seasonal anomalies (b), across-site variability (c), and interannual anomalies (d). The modeled estimates were derived from the mean ensemble of the 50 model runs. 
Such dynamic statistical modeling approach (i.e. LSTM) was expected to achieve a better performance for predicting anomalies compared to Jung et al. (2011) and Tramontana et al. (2016) analyses. In fact, LSTMs are theoretically able to automatically learn informative features (LeCun et al., 2015; Schmidhuber, 2015), and as such could capture interannual and seasonal fluctuations in the remote sensing and climate data related to specific ecosystem impact events (e.g. anthropogenic disturbances, seasonal droughts). However, this appeared not to be the case. We assume that this could be due to:

(i) the fact that anomaly signals were relatively small (Fig. 4.4b and 4.4d) compared to the low signal-to-noise ratio in the remote sensing data because of atmospheric contamination;

(ii) the non-availability of complete Landsat time series and necessary gapfilling step;

(iii) the fact that the training of the statistical models was performed at monthly scale and not at daily scale due to the temporal resolution of the Landsat data. Signatures of extreme events are likely more apparent at daily time scale, therefore one could argue that the temporal scale used in the presented study was not appropriate to capture well the anomalies in the signals;

(iv) the limitations associated with the remote sensing signals in providing all necessary information regarding vegetation structure and growth trajectory, while being insensitive to $\mathrm{C}$ decomposition dynamics;

(v) the fact that few disturbances events were observed during the observational period compared to undisturbed sites or to sites where disturbances occurred a long time ago in which no spectral recovery signals were captured during the training procedure (i.e. only $10 \%$ of the observations in recently disturbed forests); and

(vi) the lack of information related to the spatial context (e.g. landscape patchiness, fractal dimension) that could translate the development stage.

All these factors could suggest that few anomaly signals were captured during the training process of the proposed approach. Furthermore, we cannot ignore the fact that there was a lack of relevant information on the predictors used in this study to predict NEE variability across-scales. For instance, extracting temporal variation in the Landsat data could have been a good proxy for the age effects on NEE among sites, but this was not the case here (as discussed further), likely due to missing information in the input variables for the heterotrophic respiration component of NEE. The mismatch between the observed and predicted NEE at interannual scales could also be related to the fact that the training procedure did not learn site-specific characteristics due to the implemented cross-validation set-up (i.e. entire sites out cross-validation) (Bodesheim et al., 2018), therefore limiting the capacity of a statistical model to predict NEE interannual anomalies accurately. Furthermore, the cost function was performed on the monthly observations during the training/evaluation procedure, which can potentially limit the capacity of the presented approach for calculating NEE signals realistically at annual scales. In addition, there were few very young sites $(<20$ years old) or sites where disturbances occurred during the Landsat record in the 
training set, which can limit the ability of the proposed approach to have good predictive capacity in young and recently disturbed sites. Another source of uncertainty was related to the mismatch between flux measurements and the Landsat time series cutouts around each flux tower. To overcome the latter issue, integration of footprint analysis could help to better describe the origin of the fluxes within the Landsat time series cutouts.

Differences in predictive capacity were apparent for different PFTs and climate levels (Table 4.3, 4.A2, and 4.A3). The NSE for different PFTs and climate regions at the seasonal scale ranged from 0.42 (i.e. evergreen forests) to 0.82 (i.e. deciduous forests), and from -0.0006 for the tropical forests to 0.68 for both temperate and boreal forests. The fact that the LSTM showed poor agreement with observations in the tropics can be explained by the very small signal in the input data due to the lack of seasonal variation in terms of reflective, thermal, and moisture properties (Frank et al., 2015). In addition, the Landsat data tend to be very sparse in the tropics due to frequent cloud coverage, leading to a high fraction of gap-filled data, thus a potentially poor representation of the seasonal vegetation variation. The properties of the Landsat data might also not be suitable to characterize seasonality in the tropics, therefore other remote sensing products related to leaf development and demography (e.g. Multi-Angle Implementation of Atmospheric Correction (MAIAC) product) (Wu et al., 2016) could be explored. However, MAIAC products were not tested given their relatively short time series, although this shortcoming will be overcome in the future. EC flux data also have their own limitations in the tropics, not only due to sparse spatial coverage but also due to large gaps in data related to frequent rain events and severe issues with the night-time fluxes due to low wind speed and tall canopies. The LSTM was able to well predict NEE across-sites in evergreen forests ( $\mathrm{NSE}=0.43$ ), while it showed poor agreement with observations in tropical regions (NSE=-0.15). One could assume that the low number of tropical sites currently available in this dataset $(\mathrm{N}=16$ and $\mathrm{N}=7$ at the seasonal scale and across-sites, respectively) might limit an LSTM to predict spatial NEE variabilities in such an ecosystem and also led to a systematically higher uncertainty across-scales compared to other PFTs (Table 4.3).

\subsubsection{Comparison of the different model set-ups}

A comparison of the model performance was done between the different LSTM networks along with the non-dynamic statistical RF model (Table 4.4, 4.A4, 4.A5, and 4.A6). In general, the performance metrics across the model set-ups differed. All model set-ups were capable of well predicting the seasonal cycle, with the LSTM achieving particularly better model fitness and lower errors $(\mathrm{NSE}=0.66, \mathrm{RMSE}=1.12$, and MAE $=0.81$ ). Similarly, LSTM depicted better agreement between observations and predictions across-sites (NSE $=0.42$, RMSE $=0.63$, and $M A E=0.48)$. However, none of the presented model set-ups were able to successfully predict the anomaly signals, with LSTM having rather similar performance and level of errors than the other model set-ups for both seasonal $(\mathrm{NSE}=0.07$, RMSE $=0.61$, and MAE $=0.31$ ) and interannual anomalies (NSE $=0.07$, RMSE $=0.31$, and MAE $=0.22$ ). Still, this results supported 
Table 4.3: Nash-Sutcliffe modeling efficiency of the $L S T M$ setup per vegetation type and climate region from the ensemble mean \pm sd estimate of the 50 runs. Statistics for the anomalies were not calculated in the arid and tropical climate (i.e. NA) because there was no site with at least 3 years of complete data after data quality control. Savanna vegetation type includes both savanna and woody savanna sites.

\begin{tabular}{l|cccc} 
& Seasonal cycle & Seasonal anomalies & Across-sites & Interannual anomalies \\
\hline Deciduous forest & $\mathbf{0 . 8 2} \pm 0.01$ & $\mathbf{0 . 1 6} \pm 0.03$ & $\mathbf{0 . 2 6} \pm 0.07$ & $\mathbf{0 . 1 7} \pm 0.04$ \\
Evergreen forest & $\mathbf{0 . 4 2} \pm 0.02$ & $\mathbf{0 . 0 3} \pm 0.02$ & $\mathbf{0 . 4 3} \pm 0.06$ & $\mathbf{0 . 0 0 8} \pm 0.04$ \\
Mixed forest & $\mathbf{0 . 6 3} \pm 0.03$ & $\mathbf{0 . 0 1} \pm 0.02$ & $\mathbf{0 . 4 0} \pm 0.19$ & $\mathbf{0 . 0 8} \pm 0.04$ \\
Savanna & $\mathbf{0 . 5 5} \pm 0.02$ & $\mathbf{0 . 0 3} \pm 0.02$ & $\mathbf{0 . 2 9} \pm 0.48$ & $\mathbf{0 . 3 7} \pm 0.07$ \\
\hline Arid & $\mathbf{0 . 4 7} \pm 0.04$ & NA & $\mathbf{0 . 1 5} \pm 0.75$ & NA \\
Boreal & $\mathbf{0 . 6 8} \pm 0.01$ & $\mathbf{0 . 1 4} \pm 0.03$ & $\mathbf{0 . 3 3} \pm 0.07$ & $\mathbf{- 0 . 0 4} \pm 0.05$ \\
Temperate & $\mathbf{0 . 6 8} \pm 0.01$ & $\mathbf{0 . 0 4} \pm 0.02$ & $\mathbf{0 . 2 9} \pm 0.07$ & $\mathbf{0 . 0 9} \pm 0.03$ \\
Tropical & $\mathbf{- 0 . 0 0 0 6} \pm 0.12$ & NA & $\mathbf{- 0 . 1 5} \pm 0.27$ & NA
\end{tabular}

the importance of accounting for interannual and seasonal fluctuations of climate and vegetation to estimate net $\mathrm{CO}_{2}$ fluxes, in particular at the seasonal scale and across-sites. This was evidenced by $L_{S T M_{m s c}}, L S T M_{\text {perm }}, L S T M_{\text {annual }}$, and the $R F$ model set-ups, which depicted lower predictive capacities and higher errors than the original LSTM. However, these comparisons were done for the entire FLUXNET dataset, but the effects of memory appeared to be substantially different across-sites (Fig. 4.A4).

Table 4.4: Nash-Sutcliffe modeling efficiency of the proposed approach against the other model set-ups from the ensemble mean \pm sd estimate of the 50 model runs. $L S T M=$ LSTM model using the full depth of the Landsat time series and climate data; $L S T M_{\text {perm }}=L S T M$ model but the temporal patterns of both the predictive and the target variables were randomly permuted while instantaneous relationships between predictive and target variables were kept; $L S T M_{m s c}=L S T M$ model but the Landsat time series for each band were replaced by their mean seasonal cycle, while using the actual values of $\mathrm{T}_{\text {air }}$, Precip, Rg, and VPD; LSTM anпиаl $=$ LSTM model but the Landsat time series for each band were replaced by their annual mean, while using the actual values of $\mathrm{T}_{\text {air }}$, Precip, Rg, and VPD; RF = Random Forest model using the actual values of the Landsat time series and climate data.

\begin{tabular}{l|cccc} 
& Seasonal cycle & Seasonal anomalies & Across-sites & Interannual anomalies \\
\hline LSTM & $\mathbf{0 . 6 6} \pm 0.01$ & $\mathbf{0 . 0 7} \pm 0.01$ & $\mathbf{0 . 4 2} \pm 0.05$ & $\mathbf{0 . 0 7} \pm 0.03$ \\
LSTM $_{\text {msc }}$ & $\mathbf{0 . 6 4} \pm 0.009$ & $\mathbf{0 . 0 5} \pm 0.008$ & $\mathbf{0 . 3 9} \pm 0.04$ & $\mathbf{0 . 0 2} \pm 0.01$ \\
LSTM $_{\text {annual }}$ & $\mathbf{0 . 5 9} \pm 0.02$ & $\mathbf{0 . 0 6} \pm 0.03$ & $\mathbf{0 . 3 6} \pm 0.05$ & $\mathbf{0 . 0 4} \pm 0.05$ \\
LSTM $_{\text {perm }}$ & $\mathbf{0 . 6 1} \pm 0.01$ & $\mathbf{0 . 0 0 8} \pm 0.02$ & $\mathbf{0 . 3 8} \pm 0.05$ & $\mathbf{0 . 0 8} \pm 0.02$ \\
RF & $\mathbf{0 . 5 8} \pm 0.00004$ & $\mathbf{- 0 . 3 0} \pm 0.0006$ & $\mathbf{0 . 3 8} \pm 0.0002$ & $\mathbf{- 0 . 0 4} \pm 0.0007$
\end{tabular}

The fact that the LSTM network exploited the history of the predictor variables could explain its overall better results in predicting $\mathrm{CO}_{2}$ fluxes compared to other model set-ups, despite the differences being marginal. $\mathrm{CO}_{2}$ fluxes are not only linearly related to the instantaneous 
reflectance and meteorological conditions but also associated with the climate and vegetation dynamics several months to years prior (Reichstein et al., 2013), which may affect non-observed ecosystem states with direct consequences to $\mathrm{C}$ fluxes. To investigate this, an additional simulation experiment was conducted to understand how many years, before predicting a specific year, the proposed approach (i.e. LSTM) uses to achieve a better model performance (Fig. 4.5). The LSTM model trained before was used, but during the prediction, the actual values for predictors in year ${ }_{i-n}$ (where $\mathrm{n}$ is a number of years ranging from one to five) were replaced by their mean seasonal cycle when predicting year ${ }_{i}$. Hence, the interannual variations and seasonal deviations of year ${ }_{i-n}$ were not included in the predictions of the LSTMs when calculating NEE for year ${ }_{i}$. For both deciduous and evergreen forests, there was a consistent increase in the mean absolute residuals from 0 to 1 years of altered forcings, while there were no substantial changes when the number of years since alteration was $\geq 1$ year (Fig. $4.5 \mathrm{a}$ and Fig. 4.5b). It was also interesting to see that altering only climate predictors had less of an effect on the deviations from the NEE estimates, compared to the other two scenarios. For deciduous forests, capturing information from the current and the previous years resulted in the highest differences in NEE estimates mainly during the growing season (April to September) (Fig. 4.5c). On the other hand, altering the Landsat and climate time series of the previous years seemed to mainly have the highest effects on the predicted NEE from January to August-September. Overall, the magnitudes in the errors were substantially higher for deciduous forests. Note that these findings did not mean that only previous-year climate and vegetation memory effects were important for improving NEE estimates but indicated that their contribution diminished to further improve its predictive capacity in the proposed approach.

This study confirmed that changes in historical climate and vegetation dynamics played a moderate role in shaping the temporal variability of ecosystem $\mathrm{CO}_{2}$ fluxes, particularly at the seasonal scale (i.e. around $8 \%$ difference in model efficiency between $L S T M$ and $L S T M_{\text {perm }}$ ) and across-sites (i.e. 10\% difference in model efficiency between $L S T M$ and $L S T M_{\text {perm }}$ ) (Table 4.4 and Fig. 4.5). However, these findings differed markedly between forest types (Fig. 4.5 and 4.6). For instance, NEE estimates calculated by $L S T M$ and $L S T M_{m s c}$ for deciduous forests were rather similar at the seasonal scale, suggesting that the interannual variation information carried by the remote sensing data did not help to achieve better performance capacity in predicting NEE at seasonal scale in such an ecosystem, while the interannual variation in climate was still considered. On the other hand, the highest modeling efficiency was achieved for evergreen forests using the LSTM model, suggesting that both interannual and seasonal fluctuations in vegetation were important drivers of NEE variabilities at the seasonal scale.

One outstanding result of this analysis was the importance of memory effects at the seasonal scale (Table 4.4 and Fig. 4.6). Such finding can be better explored using the NEE mean seasonal variation residuals for deciduous and evergreen forests (Fig. 4.7). For deciduous and evergreen forests, it was important to extract realistic temporal vegetation and climate information when predicting NEE as the $L S T M_{\text {perm }}$ model depicted the highest overall error in the residual seasonal patterns compared to the other model set-ups. For deciduous forests, both the onset and the 
(a)

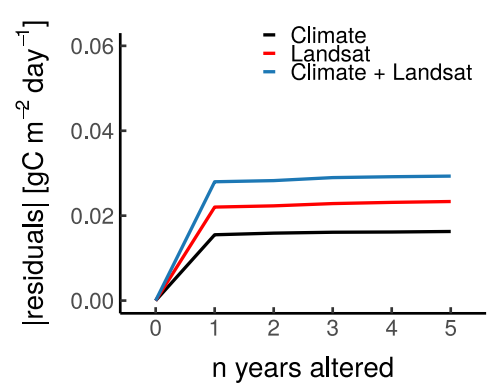

(b)

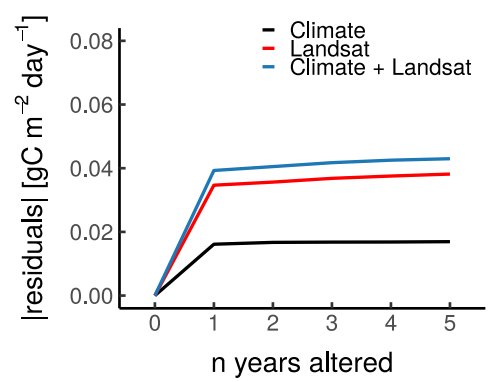

(c)
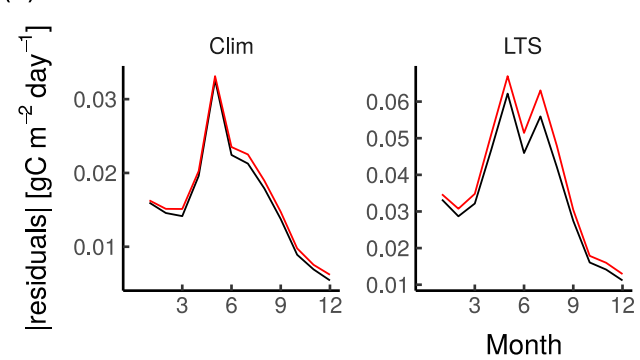

onth

(d)

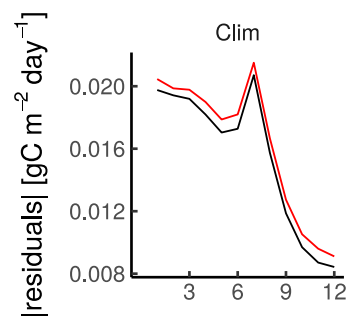

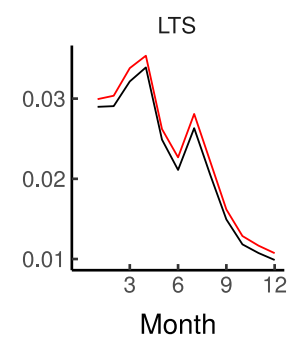

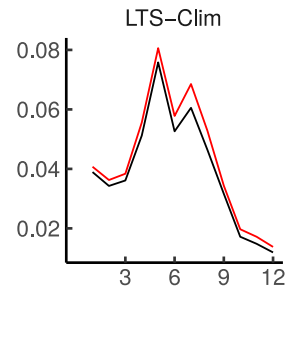

$-1-5$

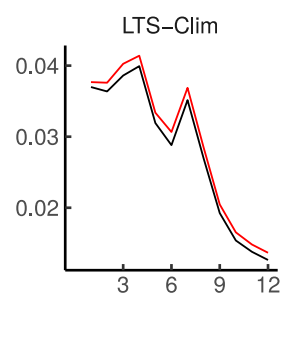

$-1-5$

Figure 4.5: Effects on predicting monthly NEE by altering $n$ year in the predictors for deciduous and evergreen forests. Average of the absolute residuals calculated between predicted monthly NEE with 0 year altered in the predictors and predicted monthly NEE with year ${ }_{i-n}$ altered in the predictors for deciduous and evergreen forests ( $a$ and $b$, respectively) is shown. The absolute residuals for the mean seasonal cycle are also reported ((c) deciduous and (d) evergreen forests)."1 year" means that only the last year was altered, "2 years" means that the last two years were altered, and so on. Months for the sites located in the Southern hemisphere have been adjusted to match the seasonal cycle of the sites in the Northern hemisphere.

peak of the growing season were better captured by $L S T M$ and $L S T M_{m s c}$ models (Fig. 4.7a). This could suggest that the climatic conditions of the previous years (e.g. water limitations (Aubinet et al., 2018; Desai, 2014), increased precipitation (Shen et al., 2016), or minimum air temperature during spring of the previous year (Zielis et al., 2014)) not only control NEE seasonal patterns in deciduous forests but also mean seasonal vegetation fluctuations. It was therefore probable that seasonal leaf physiology due to leaf aging also drived the residual seasonal patterns (Rodríguez-Calcerrada et al., 2012). The $L S T M_{\text {annual }}$ model set-up revealed 


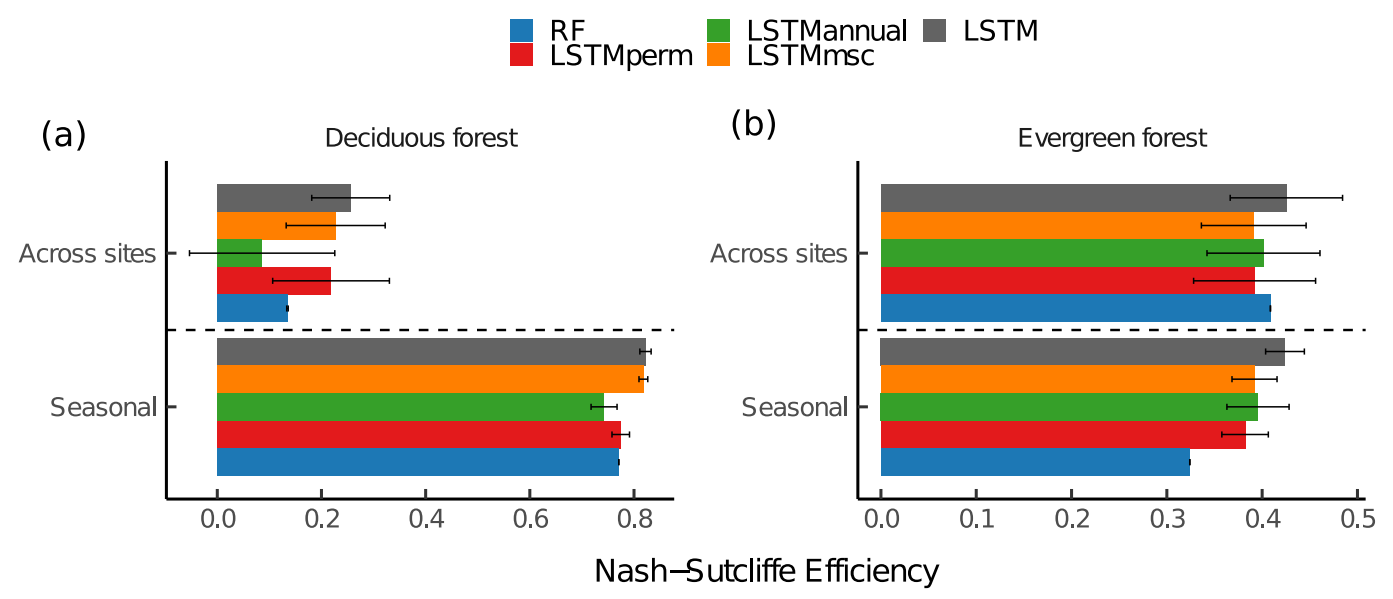

Figure 4.6: Nash-Sutcliffe modeling efficiency comparison between the proposed LSTM-based models and the other model set-ups for (a) deciduous and (b) evergreen forests. Nash-Sutcliffe modeling efficiency values have been calculated based on the mean ensemble \pm sd of the 50 model runs. $L S T M=$ LSTM model using the full depth of the Landsat time series and climate data; $L S T M_{\text {perm }}=$ LSTM model but the temporal patterns of both the predictive and the target variables were randomly permuted while instantaneous relationships between predictive and target variables were kept; $L S T M_{m s c}$ $=$ LSTM model but the Landsat time series for each band were replaced by their mean seasonal cycle, while using the actual values of $\mathrm{T}_{\text {air }}$, Precip, Rg, and VPD; $L S T M_{\text {annual }}=$ LSTM model but the Landsat time series for each band were replaced by their annual mean, while using the actual values of $\mathrm{T}_{\text {air }}$, Precip, Rg, and VPD, RF = Random Forest model using the actual values of the Landsat time series and climate data.

that capturing interannual variations in vegetation activities did not help in representing NEE estimates at the seasonal scale. However, all the model set-ups showed rather similar errors when representing the senescence phase in deciduous forests, suggesting that the processes that control these dynamics were not expressed in any of the observational datasets used here. Interestingly, LSTM, LSTM msc $_{\text {, and }}$ LSTM annual model set-ups depicted relatively similar errors over the course of the growing season for evergreen forests (Fig. 4.7b). This means that both the current climate conditions and the ones of the previous months or years control NEE seasonal cycle in such an ecosystem. These findings confirmed the existence of different ecosystem type-specific memory or lagged effects (Aubinet et al., 2018).

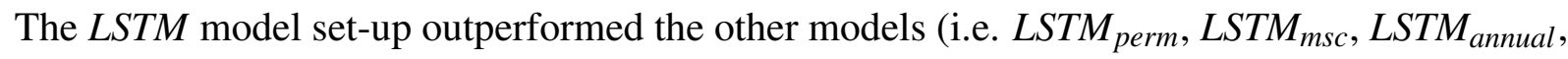
and $R F$ ) across sites, suggesting that it was able to better capture the complexity of the relation between past dynamics and current functions of the forests across-space. One hypothesis could be that net $\mathrm{CO}_{2}$ fluxes in recently disturbed forests are better predicted with a method that captures disturbance regimes. However, this hypothesis could not be confirmed since: (1) the LSTM model set-up did not outperform the other model set-ups for young forests (i.e. 0-20 years old) and for recently disturbed forests (Fig. 4.8); and (2) training an LSTM adding forest age as predictor or training it only for young forests (forest age $<40$ years) did not correct for 


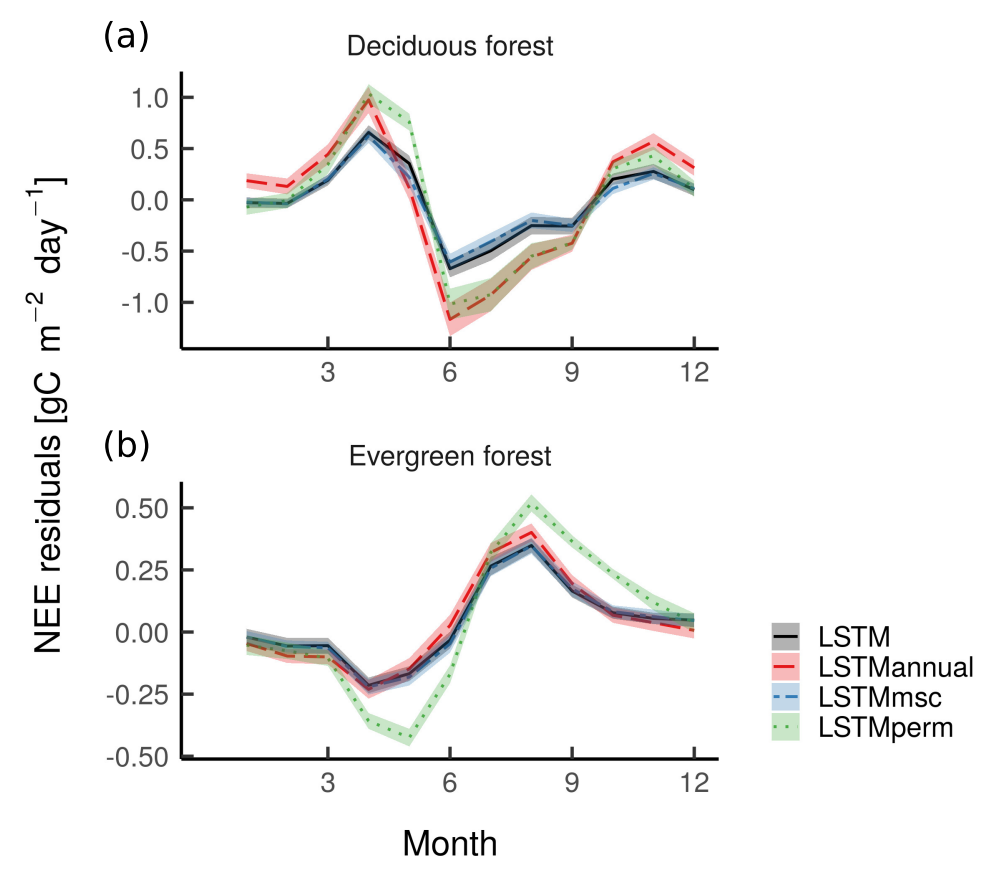

Figure 4.7: Mean seasonal variation of NEE residuals for $L S T M, L_{S T M} M_{\text {perm }}, \operatorname{LSTM}_{m s c}$, and $\boldsymbol{L S T M}_{\text {annual }}$ models for (a) deciduous and (b) evergreen forests. NEE residuals $=\left[\mathrm{NEE}\right.$ observed $_{i, j}-$ mean $\left.\left(\mathrm{NEE} \mathrm{observed}_{i}\right)\right]$ - [NEE predicted $\left.{ }_{i, j}-\operatorname{mean}\left(\mathrm{NEE} \operatorname{predicted}_{i}\right)\right]$, where $\mathrm{i}$ is a unique Fluxnet site and $\mathrm{j}$ is a monthly observation. Residual estimates have been calculated based on the mean ensemble \pm sd of the 50 model runs. $L S T M=$ LSTM model using the full depth of the Landsat time series and climate data; $L S T M_{\text {perm }}=L S T M$ model but the temporal patterns of both the predictive and the target variables were randomly permuted while instantaneous relationships between predictive and target variables were kept; $L S T M_{m s c}=L S T M$ model but the Landsat time series for each band were replaced by their mean seasonal cycle, while using the actual values of $\mathrm{T}_{\text {air }}$, Precip, Rg, and VPD; LSTM annual $=$ LSTM model but the Landsat time series for each band were replaced by their annual mean, while using the actual values of $\mathrm{T}_{\text {air }}$, Precip, Rg, and VPD; RF = Random Forest model using the actual values of the Landsat time series and climate data. Months for the sites located in the Southern hemisphere have been adjusted to match the seasonal cycle of the sites in the Northern hemisphere.

the observed bias in young forests (Fig. 4.8). However, it was not possible to be conclusive on the ability of the LSTMs to predict young sites since: (1) there was only a small sample of young forests and recently disturbed sites in this dataset; and that methodologically (2) no in-situ proxies for productivity (e.g. related to GPP) were used in the analysis; and (3) the LSTMs were trained with monthly observations. Therefore, it was very likely that the better performance of the LSTM model set-up compared to the other model set-ups at the seasonal cycle could explain its overall better capacity in explaining NEE spatial variation (e.g. spring NEE accounts for most of the annual NEE in the temperate deciduous forests (Zielis et al., 2014)). 


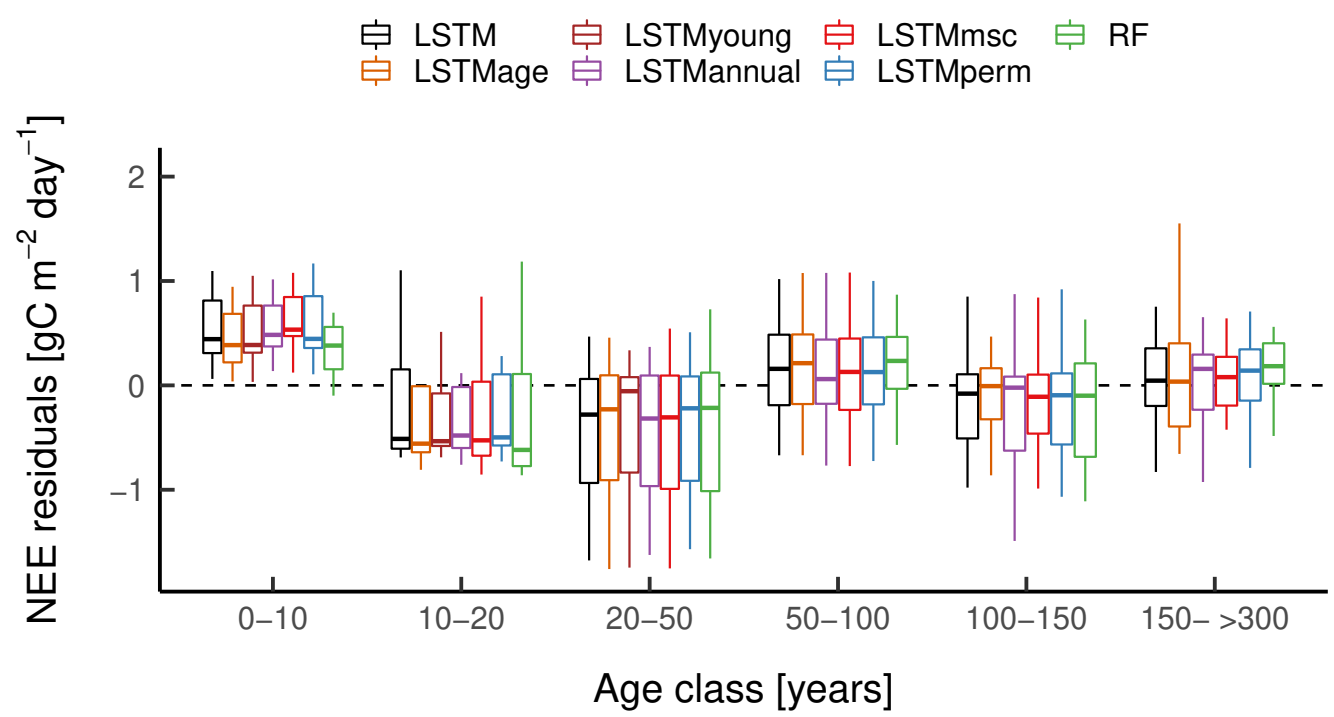

Figure 4.8: Model residuals per age class for $L S T M, L_{S T M}{ }_{\text {perm }}, L S T M_{m s c}, L S T M_{\text {annual }}$, and $R F$ models based on site-average NEE. $L S T M=$ LSTM model using the full depth of the Landsat time series and climate data; $L S T M_{\text {perm }}=L S T M$ model but the temporal patterns of both the predictive and the target variables were randomly permuted while instantaneous relationships between predictive and target variables were kept; $L S T M_{m s c}=L S T M$ model but the Landsat time series for each band were replaced by their mean seasonal cycle, while using the actual values of $\mathrm{T}_{\text {air }}$, Precip, $\mathrm{Rg}$, and VPD; $L S T M_{\text {annual }}=L S T M$ model but the Landsat time series for each band were replaced by their annual mean, while using the actual values of $\mathrm{T}_{\text {air }}$, Precip, $\mathrm{Rg}$, and VPD; RF $=$ Random Forest model using the actual values of the Landsat time series and climate data; $L S T M_{\text {age }}=L S T M+$ forest age as a predictive variable; $L S T M_{\text {young }}=L S T M$ only trained with forests younger than 40 years.

\subsection{Conclusion}

In this study, we present a novel framework for assessing the potential of the memory effects of climate and vegetation on forests' NEE using the Landsat satellite imagery and in-situ EC observations. The results presented here for the whole FLUXNET dataset reveal a variable memory effect on NEE across-scales, that is mainly apparent at the seasonal scale and across-sites. We also find that the effects of memory vary between FLUXNET sites suggesting site-specific memory effects. Although instantaneous observations of the contemporaneous vegetation states may already carry information from the past, current analysis suggests that extracting antecedent observations of vegetation and climate are beneficial for estimating NEE more realistically (the difference between $L S T M$ and $L S T M_{\text {perm }}$, as well as between $L S T M$ and $R F$ ). Such effects can emerge from the information contained in the course of the seasonal cycle or from the effects of interannual variation on NEE. However, the close agreement between LSTM and LSTM $M_{m s c}$ suggests that either the effect is smeared out by the impact of instantaneous climate on NEE or the interannual variation's memory effect in NEE is implicitly captured by this approach. The 
results are contingent on the length of observations and few recently disturbed forests but do emphasize the possibility of dynamic statistical methods that include memory effects to better estimate the contribution of forest ecosystems in the global terrestrial $\mathrm{C}$ cycle, hence for further improving statistically-based prediction methods. 


\section{A Appendix}

This appendix represents the supplementary materials of the presented publication.

\section{Sites used in this study}

Table 4.A1: List of sites used in this study. DBF $=$ Deciduous broadleaf forest, DNF $=$ deciduous needleleaf forest, $\mathrm{EBF}=$ evergreen broadleaf forest, $\mathrm{ENF}=$ evergreen needleleaf forest, $\mathrm{MF}=$ mixed forest, WSA = woody savanna, and SAV = savanna. The PFT and climate classifications used here are coming from the ancillary data files provided by the La Thuile or the FLUXNET2015 datasets (https://fluxnet.fluxdata.org).

\begin{tabular}{|c|c|c|c|c|c|}
\hline Site ID & Lat $\left[{ }^{\circ} \mathrm{N}\right]$ & Long $\left[{ }^{\circ} \mathrm{E}\right]$ & Climate type & Vegetation type & Forest age \\
\hline AR-SLu & -33.4648 & -66.4598 & Temperate & MF & 50 \\
\hline AR-Vir & -28.2395 & -56.1886 & Temperate & ENF & 8 \\
\hline AU-Ade & -13.0769 & 131.1178 & Tropical & WSA & NA \\
\hline AU-ASM & -22.2830 & 133.2490 & Arid & ENF & NA \\
\hline AU-Cpr & -34.0021 & 140.5891 & Arid & SAV & NA \\
\hline AU-Cum & -33.6133 & 150.7225 & Temperate & EBF & $>300$ \\
\hline AU-DaS & -14.1593 & 131.3881 & Tropical & SAV & NA \\
\hline AU-Dry & -15.2588 & 132.3706 & Tropical & SAV & NA \\
\hline AU-Gin & -31.3764 & 115.7138 & Temperate & WSA & NA \\
\hline AU-GWW & -30.1913 & 120.6541 & Arid & SAV & NA \\
\hline AU-How & -12.4943 & 131.1523 & Tropical & WSA & 110 \\
\hline AU-Lox & -34.4704 & 140.6551 & Arid & DBF & NA \\
\hline AU-RDF & -14.5636 & 132.4776 & Tropical & WSA & NA \\
\hline AU-Rob & -17.1175 & 145.6301 & Temperate & EBF & 198 \\
\hline AU-Tum & -35.6566 & 148.1517 & Temperate & EBF & 83 \\
\hline AU-Wac & -37.4259 & 145.1878 & Temperate & EBF & $>300$ \\
\hline AU-Whr & -36.6732 & 145.0294 & Temperate & EBF & NA \\
\hline AU-Wom & -37.4222 & 144.0944 & Temperate & EBF & 32 \\
\hline BE-Bra & 51.3092 & 4.5206 & Temperate & MF & 78 \\
\hline BE-Jal & 50.5639 & 6.0733 & Temperate & MF & 8 \\
\hline BE-Vie & 50.3051 & 5.9981 & Temperate & MF & 94 \\
\hline BR-Ban & -9.8244 & -50.1591 & Tropical & EBF & $>300$ \\
\hline BR-Cax & -1.7197 & -51.4590 & Tropical & EBF & 96 \\
\hline BR-Ji2 & -10.0832 & -61.9309 & Tropical & EBF & $>300$ \\
\hline BR-Ma2 & -2.6091 & -60.2093 & Tropical & EBF & $>300$ \\
\hline BR-Sa1 & -2.8567 & -54.9589 & Tropical & EBF & $>300$ \\
\hline BR-Sa3 & -3.0180 & -54.9714 & Tropical & EBF & $>300$ \\
\hline BR-Sp1 & -21.6195 & -47.6499 & Tropical & WSA & NA \\
\hline BW-Ma1 & -19.9155 & 23.5605 & Arid & WSA & NA \\
\hline CA-Ca1 & 49.8672 & -125.3340 & Temperate & ENF & 60 \\
\hline CA-Ca2 & 49.8705 & -125.2910 & Temperate & ENF & 3 \\
\hline CA-Ca3 & 49.5346 & -124.9000 & Temperate & ENF & 16 \\
\hline
\end{tabular}


Table 4.A1 - Continued from previous page

\begin{tabular}{|c|c|c|c|c|c|}
\hline Site ID & Lat $\left[{ }^{\circ} \mathrm{N}\right]$ & Long $\left[{ }^{\circ} \mathrm{E}\right]$ & Climate type & Vegetation type & Forest age \\
\hline CA-Gro & 48.2167 & -82.1556 & Boreal & MF & 78 \\
\hline CA-Man & 55.8796 & -98.4808 & Boreal & ENF & 161 \\
\hline CA-NS1 & 55.8792 & -98.4839 & Boreal & ENF & 154 \\
\hline CA-NS2 & 55.9058 & -98.5247 & Boreal & ENF & 73 \\
\hline CA-NS3 & 55.9117 & -98.3822 & Boreal & ENF & 39 \\
\hline CA-NS4 & 55.9144 & -98.3806 & Boreal & ENF & 39 \\
\hline CA-NS5 & 55.8631 & -98.4850 & Boreal & ENF & 23 \\
\hline CA-Oas & 53.6289 & -106.1978 & Boreal & $\mathrm{DBF}$ & 80 \\
\hline CA-Obs & 53.9872 & -105.1178 & Boreal & ENF & 112 \\
\hline CA-Ojp & 53.9163 & -104.6920 & Boreal & ENF & 88 \\
\hline CA-Qcu & 49.2671 & -74.0365 & Boreal & ENF & 4 \\
\hline CA-Qfo & 49.6925 & -74.3421 & Boreal & ENF & 102 \\
\hline CA-SF1 & 54.4850 & -105.8176 & Boreal & ENF & 28 \\
\hline CA-SF2 & 54.2539 & -105.8775 & Boreal & ENF & 14 \\
\hline CA-SJ1 & 53.9080 & -104.6560 & Boreal & ENF & 10 \\
\hline $\mathrm{CA}-\mathrm{SJ} 2$ & 53.9450 & -104.6490 & Boreal & ENF & 2 \\
\hline CA-SJ3 & 53.8758 & -104.6450 & Boreal & ENF & 30 \\
\hline CA-TP1 & 42.6609 & -80.5595 & Boreal & ENF & 9 \\
\hline CA-TP2 & 42.7744 & -80.4588 & Boreal & ENF & 73 \\
\hline CA-TP3 & 42.7068 & -80.3483 & Boreal & ENF & 37 \\
\hline CA-TP4 & 42.7102 & -80.3574 & Boreal & ENF & 70 \\
\hline CA-TPD & 42.6353 & -80.5577 & Boreal & $\mathrm{DBF}$ & 98 \\
\hline CG-Hin & -4.6811 & 12.0036 & Tropical & $\mathrm{EBF}$ & NA \\
\hline CG-Kis & -4.7914 & 11.9822 & Tropical & $\mathrm{EBF}$ & NA \\
\hline CG-Tch & -4.2892 & 11.6564 & Tropical & SAV & NA \\
\hline CH-Dav & 46.8153 & 9.8559 & Temperate & ENF & 222 \\
\hline CH-Lae & 47.4781 & 8.3650 & Temperate & $\mathrm{MF}$ & 184 \\
\hline CN-Anh & 33.0000 & 117.0000 & Temperate & $\mathrm{DBF}$ & 13 \\
\hline CN-Bed & 39.5306 & 116.2520 & Boreal & $\mathrm{EBF}$ & 12 \\
\hline CN-Cha & 42.4025 & 128.0958 & Boreal & $\mathrm{MF}$ & $>300$ \\
\hline CN-Din & 23.1733 & 112.5361 & Temperate & $\mathrm{EBF}$ & 96 \\
\hline CN-Hny & 29.3100 & 112.5100 & Temperate & $\mathrm{DBF}$ & 2 \\
\hline $\mathrm{CN}-\mathrm{Ku} 1$ & 40.5383 & 108.6940 & Arid & $\mathrm{EBF}$ & 5 \\
\hline CN-Qia & 26.7414 & 115.0581 & Temperate & ENF & 19 \\
\hline CZ-BK1 & 49.5021 & 18.5369 & Boreal & ENF & 31 \\
\hline DE-Bay & 50.1419 & 11.8669 & Temperate & ENF & 54 \\
\hline DE-Hai & 51.0792 & 10.4530 & Temperate & $\mathrm{DBF}$ & 254 \\
\hline DE-Har & 47.9344 & 7.6010 & Temperate & ENF & 42 \\
\hline DE-Lkb & 49.0996 & 13.3047 & Temperate & ENF & 2 \\
\hline DE-Lnf & 51.3282 & 10.3678 & Temperate & DBF & 117 \\
\hline DE-Meh & 51.2753 & 10.6555 & Temperate & $\mathrm{MF}$ & 2 \\
\hline DE-Obe & 50.7836 & 13.7196 & Temperate & ENF & 76 \\
\hline DE-Tha & 50.9636 & 13.5669 & Temperate & ENF & 118 \\
\hline DE-Wet & 50.4535 & 11.4575 & Temperate & ENF & 54 \\
\hline DK-Sor & 55.4859 & 11.6446 & Temperate & DBF & 85 \\
\hline ES-ES1 & 39.3460 & -0.3188 & Temperate & ENF & 116 \\
\hline ES-LMa & 39.9415 & -5.7734 & Temperate & SAV & 148 \\
\hline
\end{tabular}

Continued on next page 
Table 4.A1 - Continued from previous page

\begin{tabular}{|c|c|c|c|c|c|}
\hline Site ID & Lat $\left[{ }^{\circ} \mathrm{N}\right]$ & Long $\left[{ }^{\circ} \mathrm{E}\right]$ & Climate type & Vegetation type & Forest age \\
\hline FI-Hyy & 61.8475 & 24.2950 & Boreal & ENF & 46 \\
\hline FI-Let & 60.6418 & 23.9597 & Boreal & ENF & NA \\
\hline FI-Sod & 67.3619 & 26.6378 & Boreal & ENF & 161 \\
\hline FR-Fon & 48.4764 & 2.7801 & Temperate & $\mathrm{DBF}$ & 150 \\
\hline FR-Hes & 48.6742 & 7.0646 & Temperate & $\mathrm{DBF}$ & 37 \\
\hline FR-LBr & 44.7171 & -0.7693 & Temperate & ENF & 34 \\
\hline FR-Pue & 43.7414 & 3.5958 & Temperate & $\mathrm{EBF}$ & 64 \\
\hline GF-Guy & 5.2788 & -52.9249 & Tropical & $\mathrm{EBF}$ & $>300$ \\
\hline GH-Ank & 5.2685 & -2.6942 & Tropical & $\mathrm{EBF}$ & NA \\
\hline IL-Yat & 31.3450 & 35.0515 & Arid & ENF & 39 \\
\hline IS-Gun & 63.8333 & -20.2167 & Temperate & DBF & 7 \\
\hline IT-Bon & 39.4778 & 16.5347 & Temperate & ENF & 36 \\
\hline IT-CA1 & 42.3804 & 12.0266 & Temperate & $\mathrm{DBF}$ & NA \\
\hline IT-CA3 & 42.3800 & 12.0222 & Temperate & DBF & NA \\
\hline IT-Col & 41.8494 & 13.5881 & Temperate & $\mathrm{DBF}$ & 180 \\
\hline IT-Cp2 & 41.7043 & 12.3573 & Temperate & $\mathrm{EBF}$ & 63 \\
\hline IT-Cpz & 41.7052 & 12.3761 & Temperate & $\mathrm{EBF}$ & 56 \\
\hline IT-Isp & 45.8126 & 8.6336 & Temperate & $\mathrm{DBF}$ & NA \\
\hline IT-Lec & 43.3046 & 11.2706 & Temperate & $\mathrm{EBF}$ & NA \\
\hline IT-LMa & 45.5813 & 7.1546 & Temperate & $\mathrm{DBF}$ & 71 \\
\hline IT-Non & 44.6898 & 11.0887 & Temperate & $\mathrm{MF}$ & 10 \\
\hline IT-PT1 & 45.2009 & 9.0610 & Temperate & DBF & 13 \\
\hline IT-Ren & 46.5869 & 11.4337 & Boreal & ENF & 188 \\
\hline IT-Ro1 & 42.4081 & 11.9300 & Temperate & $\mathrm{DBF}$ & 10 \\
\hline IT-Ro2 & 42.3903 & 11.9209 & Temperate & DBF & 19 \\
\hline IT-SR2 & 43.7320 & 10.2910 & Temperate & ENF & 64 \\
\hline IT-SRo & 43.7279 & 10.2844 & Temperate & ENF & 54 \\
\hline IT-Vig & 45.3167 & 8.8500 & Temperate & DBF & 15 \\
\hline JP-MBF & 44.3869 & 142.3186 & Boreal & $\mathrm{DBF}$ & NA \\
\hline JP-SMF & 35.2617 & 137.0788 & Temperate & $\mathrm{MF}$ & NA \\
\hline JP-Tak & 36.1462 & 137.4230 & Boreal & $\mathrm{DBF}$ & 72 \\
\hline JP-Tef & 45.0563 & 142.1062 & Boreal & MF & 121 \\
\hline JP-Tom & 42.7395 & 141.5149 & Boreal & $\mathrm{MF}$ & 48 \\
\hline ML-Kem & 15.2237 & -1.5662 & Arid & DBF & NA \\
\hline MY-PSO & 2.9730 & 102.3062 & Tropical & $\mathrm{EBF}$ & 106 \\
\hline NL-Loo & 52.1666 & 5.7436 & Temperate & ENF & 106 \\
\hline PA-SPn & 9.3181 & -79.6346 & Tropical & $\mathrm{DBF}$ & 7 \\
\hline PT-Esp & 38.6394 & -8.6018 & Temperate & $\mathrm{EBF}$ & 12 \\
\hline PT-Mi1 & 38.5407 & -8.0004 & Temperate & $\mathrm{EBF}$ & 88 \\
\hline RU-Fyo & 56.4615 & 32.9221 & Boreal & ENF & 236 \\
\hline RU-SkP & 62.2550 & 129.1680 & Boreal & DNF & 161 \\
\hline RU-Zot & 60.8008 & 89.3508 & Boreal & ENF & 201 \\
\hline SD-Dem & 13.2829 & 30.4783 & Arid & SAV & NA \\
\hline SE-Abi & 68.3624 & 18.7948 & Tundra & DBF & NA \\
\hline SE-Fla & 64.1128 & 19.4569 & Boreal & ENF & 37 \\
\hline SE-Nor & 60.0865 & 17.4795 & Boreal & ENF & 105 \\
\hline SE-Sk1 & 60.1250 & 17.9181 & Boreal & ENF & 2 \\
\hline
\end{tabular}

Continued on next page 
Table 4.A1 - Continued from previous page

\begin{tabular}{|c|c|c|c|c|c|}
\hline Site ID & Lat $\left[{ }^{\circ} \mathrm{N}\right]$ & Long $\left[{ }^{\circ} \mathrm{E}\right]$ & Climate type & Vegetation type & Forest age \\
\hline SE-Sk2 & 60.1297 & 17.8401 & Boreal & ENF & 33 \\
\hline SK-Tat & 49.1208 & 20.1635 & Boreal & ENF & 0 \\
\hline SN-Dhr & 15.4028 & -15.4322 & Arid & SAV & NA \\
\hline UK-Gri & 56.6072 & -3.7981 & Temperate & ENF & 21 \\
\hline UK-Ham & 51.1208 & -0.8608 & Temperate & $\mathrm{DBF}$ & 64 \\
\hline UK-PL3 & 51.4500 & -1.2667 & Temperate & $\mathrm{DBF}$ & NA \\
\hline US-Bar & 44.0646 & -71.2881 & Boreal & $\mathrm{DBF}$ & 128 \\
\hline US-Blo & 38.8953 & -120.6328 & Temperate & ENF & 13 \\
\hline US-Bn1 & 63.9198 & -145.3780 & Boreal & ENF & 83 \\
\hline US-Bn2 & 63.9198 & -145.3780 & Boreal & $\mathrm{DBF}$ & 16 \\
\hline US-Dk2 & 35.9736 & -79.1004 & Temperate & DBF & 98 \\
\hline US-Dk3 & 35.9782 & -79.0942 & Temperate & ENF & 21 \\
\hline US-Fmf & 35.1426 & -111.7273 & Temperate & ENF & 150 \\
\hline US-FR2 & 29.9495 & -97.9962 & Temperate & WSA & NA \\
\hline US-Fuf & 35.0890 & -111.7620 & Temperate & ENF & 101 \\
\hline US-GBT & 41.3658 & -106.2397 & Boreal & ENF & 176 \\
\hline US-GLE & 41.3665 & -106.2399 & Boreal & ENF & 184 \\
\hline US-Ha1 & 42.5378 & -72.1715 & Boreal & $\mathrm{DBF}$ & 96 \\
\hline US-Ha2 & 42.5393 & -72.1779 & Boreal & ENF & 91 \\
\hline US-Ho1 & 45.2041 & -68.7402 & Boreal & ENF & 206 \\
\hline US-Ho2 & 45.2091 & -68.7470 & Boreal & ENF & 208 \\
\hline US-KS1 & 28.4583 & -80.6709 & Temperate & ENF & 7 \\
\hline US-LPH & 42.5419 & -72.1850 & Boreal & DBF & 98 \\
\hline US-Me1 & 44.5794 & -121.5000 & Temperate & ENF & 2 \\
\hline US-Me2 & 44.4523 & -121.5574 & Temperate & ENF & 94 \\
\hline US-Me3 & 44.3154 & -121.6078 & Temperate & ENF & 20 \\
\hline US-Me4 & 44.4992 & -121.6224 & Temperate & ENF & 24 \\
\hline US-Me5 & 44.4372 & -121.5668 & Temperate & ENF & 22 \\
\hline US-Me6 & 44.3233 & -121.6078 & Temperate & ENF & 22 \\
\hline US-MMS & 39.3232 & -86.4131 & Temperate & $\mathrm{DBF}$ & 95 \\
\hline US-MOz & 38.7441 & -92.2000 & Temperate & $\mathrm{DBF}$ & 78 \\
\hline US-NC2 & 35.8031 & -76.6679 & Temperate & ENF & 14 \\
\hline US-NR1 & 40.0329 & -105.5464 & Boreal & ENF & 110 \\
\hline US-Oho & 41.5545 & -83.8438 & Boreal & DBF & 50 \\
\hline US-PFa & 45.9459 & -90.2723 & Boreal & $\mathrm{MF}$ & 150 \\
\hline US-Prr & 65.1237 & -147.4876 & Boreal & ENF & 98 \\
\hline US-SP1 & 29.7381 & -82.2188 & Temperate & ENF & 63 \\
\hline US-SP2 & 29.7648 & -82.2448 & Temperate & ENF & 4 \\
\hline US-SP3 & 29.7548 & -82.1633 & Temperate & ENF & 12 \\
\hline US-SP4 & 29.8028 & -82.2031 & Temperate & ENF & 0 \\
\hline US-SRM & 31.8214 & -110.8661 & Arid & WSA & 201 \\
\hline US-Syv & 46.2420 & -89.3477 & Boreal & $\mathrm{MF}$ & $>300$ \\
\hline US-Ton & 38.4316 & -120.9660 & Temperate & WSA & NA \\
\hline US-UMB & 45.5598 & -84.7138 & Boreal & DBF & 93 \\
\hline US-UMd & 45.5625 & -84.6975 & Boreal & DBF & 90 \\
\hline US-WBW & 35.9588 & -84.2874 & Temperate & $\mathrm{DBF}$ & 110 \\
\hline US-WCr & 45.8059 & -90.0799 & Boreal & DBF & 96 \\
\hline
\end{tabular}

Continued on next page 
Table 4.A1 - Continued from previous page

\begin{tabular}{cccccc}
\hline Site ID & Lat $\left[{ }^{\circ} \mathrm{N}\right]$ & Long $\left[{ }^{\circ} \mathrm{E}\right]$ & Climate type & Vegetation type & Forest age \\
\hline US-Wi0 & 46.6188 & -91.0814 & Boreal & ENF & 7 \\
US-Wi1 & 46.7305 & -91.2329 & Boreal & DBF & 15 \\
US-Wi3 & 46.6347 & -91.0987 & Boreal & DBF & 66 \\
US-Wi4 & 46.7393 & -91.1663 & Boreal & ENF & 66 \\
US-Wi5 & 46.6531 & -91.0858 & Boreal & ENF & 9 \\
US-Wi8 & 46.7223 & -91.2524 & Boreal & DBF & 2 \\
US-Wi9 & 46.6188 & -91.0814 & Boreal & ENF & 16 \\
US-Wrc & 45.8205 & -121.9520 & Temperate & ENF & $>300$ \\
VU-Coc & -15.4427 & 167.1920 & Tropical & EBF & 20 \\
ZA-Kru & -25.0197 & 31.4969 & Temperate & SAV & NA \\
ZA-Map & -23.8325 & 31.2144 & Temperate & SAV & NA \\
ZM-Mon & -15.4378 & 23.2528 & Tropical & WSA & 88 \\
\hline
\end{tabular}

RMSE and MAE of the the LSTM setup per PFT and climate region.

Table 4.A2: RMSE of the LSTM setup per PFT and climate region from the ensemble mean mean \pm sd estimate of the 50 runs. Statistics for the anomalies were not calculated in the arid and tropical climate (i.e. NA) because there was no site with at least 2 years of complete data after data quality control.

\begin{tabular}{l|cccc} 
& Seasonal cycle & Seasonal anomaly & Across-site & Interannual anomaly \\
\hline Deciduous forest & $\mathbf{1 . 1 8} \pm 0.03$ & $\mathbf{0 . 6 1} \pm 0.01$ & $\mathbf{0 . 5 1} \pm 0.02$ & $\mathbf{0 . 2 7} \pm 0.007$ \\
Evergreen forest & $\mathbf{1 . 1 5} \pm 0.02$ & $\mathbf{0 . 5 8} \pm 0.006$ & $\mathbf{0 . 7 2} \pm 0.03$ & $\mathbf{0 . 3 1} \pm 0.006$ \\
Mixed forest & $\mathbf{1 . 1 0} \pm 0.05$ & $\mathbf{0 . 6 8} \pm 0.007$ & $\mathbf{0 . 5 0} \pm 0.08$ & $\mathbf{0 . 3 7} \pm 0.008$ \\
Savanna & $\mathbf{0 . 7 5} \pm 0.02$ & $\mathbf{0 . 5 8} \pm 0.007$ & $\mathbf{0 . 3 4} \pm 0.09$ & $\mathbf{0 . 1 9} \pm 0.01$ \\
\hline Arid & $\mathbf{0 . 7 1} \pm 0.03$ & NA & $\mathbf{0 . 2 0} \pm 0.07$ & NA \\
Boreal & $\mathbf{1 . 0 2} \pm 0.02$ & $\mathbf{0 . 4 5} \pm 0.006$ & $\mathbf{0 . 5 2} \pm 0.03$ & $\mathbf{0 . 1 8} \pm 0.004$ \\
Temperate & $\mathbf{1 . 1 8} \pm 0.02$ & $\mathbf{0 . 7 1} \pm 0.006$ & $\mathbf{0 . 6 5} \pm 0.03$ & $\mathbf{0 . 3 8} \pm 0.006$ \\
Tropical & $\mathbf{1 . 4 0} \pm 0.08$ & NA & $\mathbf{1 . 0 3} \pm 0.12$ & NA
\end{tabular}


Table 4.A3: MAE of the LSTM setup per PFT and climate region from the ensemble mean mean \pm sd estimate of the 50 runs. Statistics for the anomalies were not calculated in the arid and tropical climate (i.e. NA) because there was no site with at least 2 years of complete data after data quality control.

\begin{tabular}{l|cccc} 
& Seasonal cycle & Seasonal anomaly & Across-site & Interannual anomaly \\
\hline Deciduous forest & $\mathbf{0 . 8 6} \pm 0.03$ & $\mathbf{0 . 4 2} \pm 0.007$ & $\mathbf{0 . 4 5} \pm 0.02$ & $\mathbf{0 . 2 0} \pm 0.006$ \\
Evergreen forest & $\mathbf{0 . 8 3} \pm 0.02$ & $\mathbf{0 . 4 0} \pm 0.004$ & $\mathbf{0 . 5 4} \pm 0.03$ & $\mathbf{0 . 2 0} \pm 0.004$ \\
Mixed forest & $\mathbf{0 . 7 8} \pm 0.05$ & $\mathbf{0 . 4 7} \pm 0.006$ & $\mathbf{0 . 4 3} \pm 0.05$ & $\mathbf{0 . 2 9} \pm 0.006$ \\
Savanna & $\mathbf{0 . 5 7} \pm 0.02$ & $\mathbf{0 . 4 6} \pm 0.007$ & $\mathbf{0 . 2 2} \pm 0.06$ & $\mathbf{0 . 1 6} \pm 0.01$ \\
\hline Arid & $\mathbf{0 . 5 0} \pm 0.03$ & NA & $\mathbf{0 . 1 6} \pm 0.06$ & NA \\
Boreal & $\mathbf{0 . 6 8} \pm 0.02$ & $\mathbf{0 . 3 0} \pm 0.004$ & $\mathbf{0 . 3 7} \pm 0.02$ & $\mathbf{0 . 1 4} \pm 0.003$ \\
Temperate & $\mathbf{0 . 8 9} \pm 0.02$ & $\mathbf{0 . 5 1} \pm 0.005$ & $\mathbf{0 . 5 4} \pm 0.02$ & $\mathbf{0 . 2 8} \pm 0.005$ \\
Tropical & $\mathbf{1 . 1 2} \pm 0.07$ & NA & $\mathbf{0 . 8 5} \pm 0.10$ & NA
\end{tabular}


Comparison of Coefficient of determination, RMSE, and MAE between the the different model setups

Table 4.A4: Coefficient of determination of the proposed approach against the other model setups from the ensemble mean mean \pm sd estimate of the 50 runs. $L S T M=$ LSTM model using the full depth of the Landsat time series and climate data; $L S T M_{\text {perm }}=L S T M$ model but the temporal patterns of both the predictive and the target variables were randomly permuted while instantaneous relationships between predictive and target variables were kept; $L S T M_{m s c}=L S T M$ model but the Landsat time series for each band were replaced by their mean seasonal cycle, while using the actual values of $\mathrm{T}_{\text {air }}$, Precip, Rg, and VPD; LSTM annual $=$ LSTM model but the Landsat time series for each band were replaced by their annual mean, while using the actual values of $\mathrm{T}_{\text {air }}$, Precip, $\mathrm{Rg}$, and VPD; RF = Random Forest model using the actual values of the Landsat time series and climate data.

\begin{tabular}{l|cccc} 
& Seasonal & Seasonal anomaly & Across-site & Interannual anomaly \\
\hline LSTM & $\mathbf{0 . 6 6} \pm 0.01$ & $\mathbf{0 . 1 0} \pm 0.006$ & $\mathbf{0 . 4 3} \pm 0.04$ & $\mathbf{0 . 0 9} \pm 0.02$ \\
LSTM $_{\text {msc }}$ & $\mathbf{0 . 6 4} \pm 0.01$ & $\mathbf{0 . 0 5} \pm 0.006$ & $\mathbf{0 . 4 0} \pm 0.04$ & $\mathbf{0 . 0 2} \pm 0.008$ \\
LSTM $_{\text {annиаl }}$ & $\mathbf{0 . 6 0} \pm 0.02$ & $\mathbf{0 . 0 7} \pm 0.008$ & $\mathbf{0 . 3 7} \pm 0.04$ & $\mathbf{0 . 0 7} \pm 0.01$ \\
LSTM $_{\text {perm }}$ & $\mathbf{0 . 6 2} \pm 0.01$ & $\mathbf{0 . 0 8} \pm 0.005$ & $\mathbf{0 . 3 9} \pm 0.04$ & $\mathbf{0 . 1 1} \pm 0.02$ \\
RF & $\mathbf{0 . 5 8} \pm 0.00003$ & $\mathbf{0 . 0 6} \pm 0.00003$ & $\mathbf{0 . 3 9} \pm 0.0001$ & $\mathbf{0 . 0 7} \pm 0.0004$
\end{tabular}

Table 4.A5: RMSE of the proposed approach against the other model setups from the ensemble mean mean \pm sd estimate of the 50 runs. $L S T M=$ LSTM model using the full depth of the Landsat time series and climate data; $L S T M_{\text {perm }}=L S T M$ model but the temporal patterns of both the predictive and the target variables were randomly permuted while instantaneous relationships between predictive and target variables were kept; $L S T M_{m s c}=L S T M$ model but the Landsat time series for each band were replaced by their mean seasonal cycle, while using the actual values of $\mathrm{T}_{\text {air }}$, Precip, $\mathrm{Rg}$, and VPD; $L S T M_{\text {annual }}=L S T M$ model but the Landsat time series for each band were replaced by their annual mean, while using the actual values of $\mathrm{T}_{\text {air }}$, Precip, $\mathrm{Rg}$, and VPD; $\mathrm{RF}=$ Random Forest model using the actual values of the Landsat time series and climate data.

\begin{tabular}{l|cccc} 
& Seasonal & Seasonal anomaly & Across-site & Interannual anomaly \\
\hline LSTM & $\mathbf{1 . 1 2} \pm 0.02$ & $\mathbf{0 . 6 1} \pm 0.005$ & $\mathbf{0 . 6 3} \pm 0.02$ & $\mathbf{0 . 3 1} \pm 0.004$ \\
LSTM $_{\text {msc }}$ & $\mathbf{1 . 1 5} \pm 0.01$ & $\mathbf{0 . 6 1} \pm 0.003$ & $\mathbf{0 . 6 5} \pm 0.02$ & $\mathbf{0 . 3 2} \pm 0.002$ \\
LSTM $_{\text {пnпиаl }}$ & $\mathbf{1 . 2 3} \pm 0.03$ & $\mathbf{0 . 6 1} \pm 0.008$ & $\mathbf{0 . 6 6} \pm 0.02$ & $\mathbf{0 . 3 2} \pm 0.008$ \\
LSTM $_{\text {perm }}$ & $\mathbf{1 . 1 9} \pm 0.02$ & $\mathbf{0 . 6 3} \pm 0.005$ & $\mathbf{0 . 6 5} \pm 0.03$ & $\mathbf{0 . 3 1} \pm 0.004$ \\
RF & $\mathbf{1 . 2 5} \pm 0.00006$ & $\mathbf{0 . 7 2} \pm 0.0002$ & $\mathbf{0 . 6 5} \pm 0.0001$ & $\mathbf{0 . 3 3} \pm 0.0001$
\end{tabular}


Table 4.A6: MAE of the proposed approach against the other model set-ups from the ensemble mean mean \pm sd estimate of the 50 runs. $L S T M=$ LSTM model using the full depth of the Landsat time series and climate data; $L S T M_{\text {perm }}=L S T M$ model but the temporal patterns of both the predictive and the target variables were randomly permuted while instantaneous relationships between predictive and target variables were kept; $L S T M_{m s c}=L S T M$ model but the Landsat time series for each band were replaced by their mean seasonal cycle, while using the actual values of $\mathrm{T}_{\text {air }}$, Precip, $\mathrm{Rg}$, and VPD; $L S T M_{\text {annual }}=L S T M$ model but the Landsat time series for each band were replaced by their annual mean, while using the actual values of $\mathrm{T}_{\text {air }}$, Precip, $\mathrm{Rg}$, and VPD; $\mathrm{RF}=$ Random Forest model using the actual values of the Landsat time series and climate data.

\begin{tabular}{l|cccc} 
& Seasonal & Seasonal anomaly & Across-site & Interannual anomaly \\
\hline LSTM & $\mathbf{0 . 8 1} \pm 0.01$ & $\mathbf{0 . 4 2} \pm 0.003$ & $\mathbf{0 . 4 8} \pm 0.02$ & $\mathbf{0 . 2 2} \pm 0.003$ \\
LSTM $_{\text {msc }}$ & $\mathbf{0 . 8 3} \pm 0.01$ & $\mathbf{0 . 4 2} \pm 0.002$ & $\mathbf{0 . 5 0} \pm 0.02$ & $\mathbf{0 . 2 2} \pm 0.002$ \\
LSTM $_{\text {nnпиal }}$ & $\mathbf{0 . 8 9} \pm 0.02$ & $\mathbf{0 . 4 2} \pm 0.006$ & $\mathbf{0 . 5 1} \pm 0.02$ & $\mathbf{0 . 2 2} \pm 0.006$ \\
LSTM $_{\text {perm }}$ & $\mathbf{0 . 8 6} \pm 0.01$ & $\mathbf{0 . 4 3} \pm 0.003$ & $\mathbf{0 . 5 0} \pm 0.02$ & $\mathbf{0 . 2 2} \pm 0.003$ \\
RF & $\mathbf{0 . 9 1} \pm 0.00004$ & $\mathbf{0 . 5 1} \pm 0.00008$ & $\mathbf{0 . 5 1} \pm 0.0001$ & $\mathbf{0 . 2 4} \pm 0.00009$
\end{tabular}




\section{Performance of the gap-filling procedure of each Landsat band}

(a)

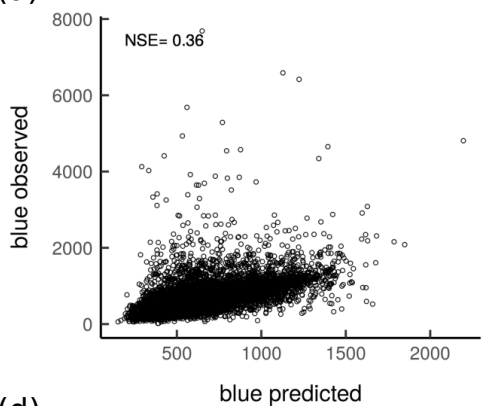

(d)

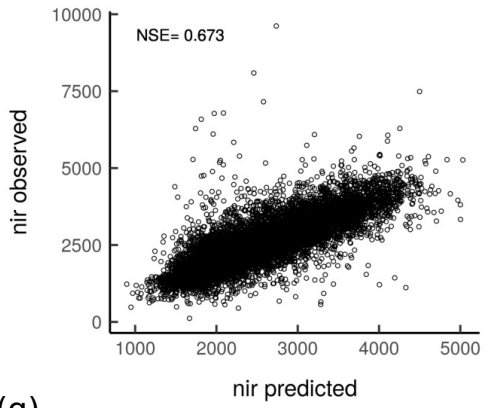

(g)

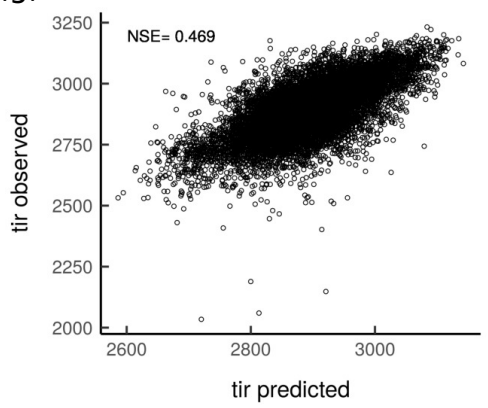

(b)

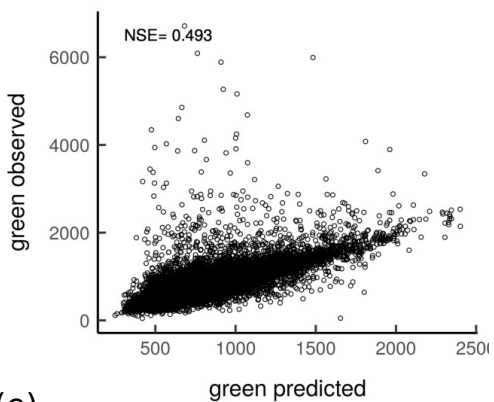

(e)

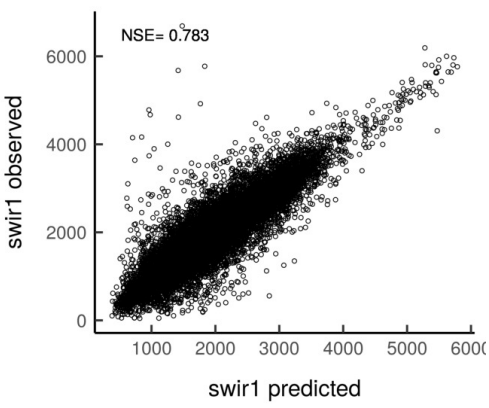

(c)

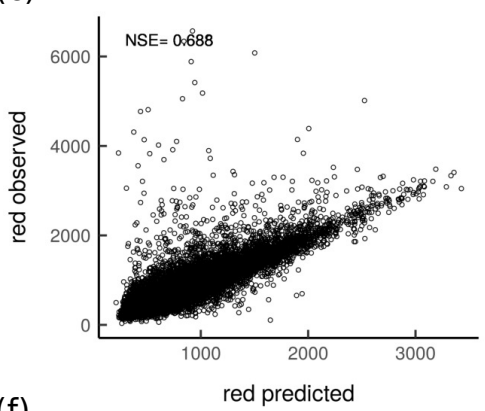

(f)

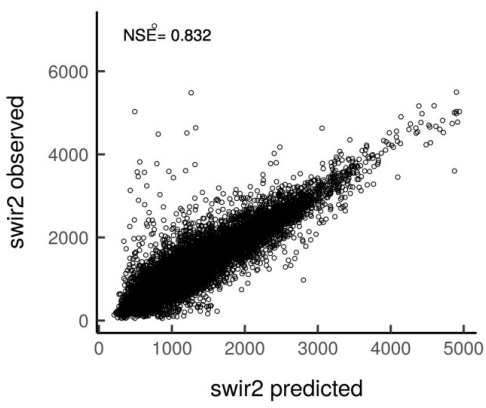

Figure 4.A1: Performance of the gap-filling procedure of each Landsat band using a RF model and the MODIS bands as predictive variables. The model was trained on $70 \%$ of the data and evaluated on $30 \%$ of the left out data. nir $=$ near-infrared, swir $1=$ shortwave infrared 1 , swir $2=$ shortwave infrared 2, and tir $=$ thermal infrared. 
(a)

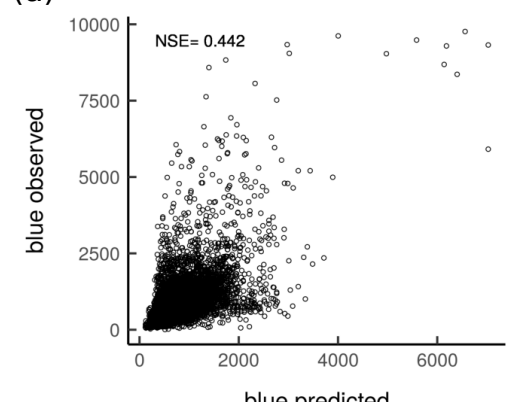

(d)

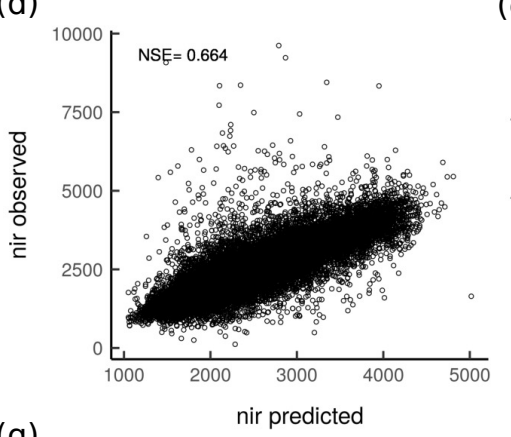

(g)

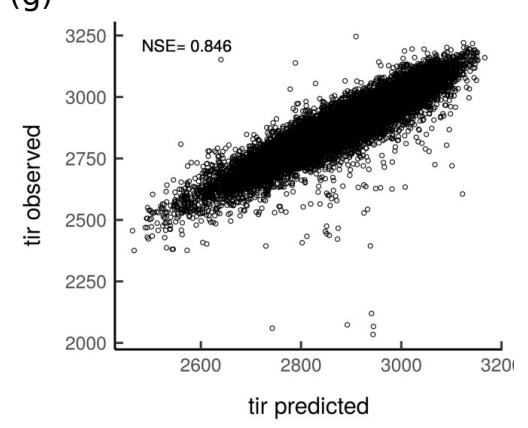

(b)

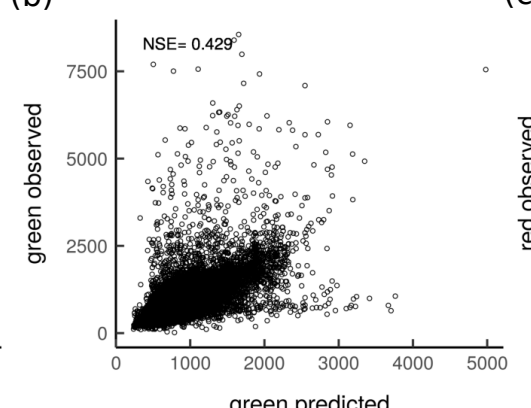

(e)

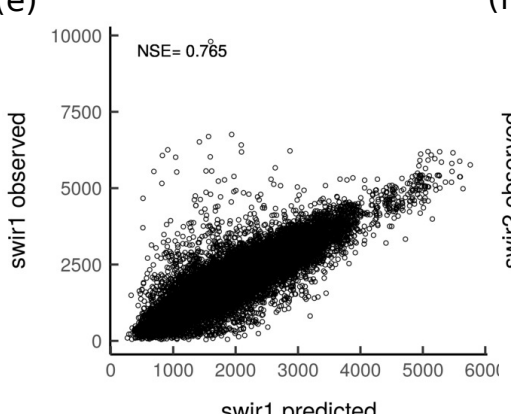

(c)

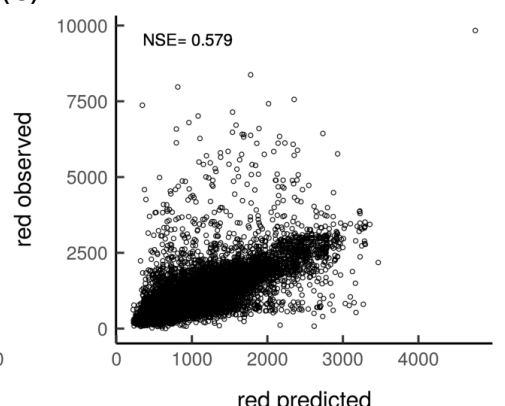

(f)

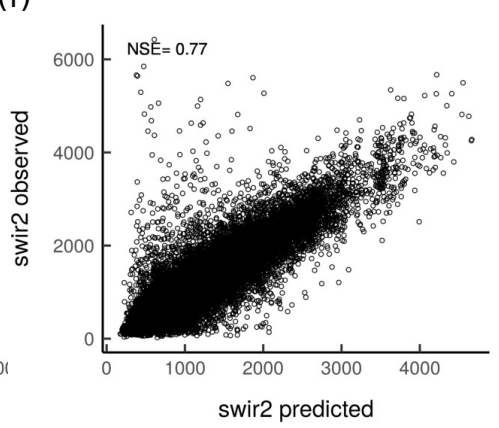

Figure 4.A2: Performance of the gap-filling procedure of each Landsat band using a Random Forest model and climate variables, PFT, latitude, and month as predictive variables. The model was trained on $70 \%$ of the data and evaluated on $30 \%$ of the left out data. nir $=$ near-infrared, swir $1=$ shortwave infrared 1 , swir $2=$ shortwave infrared 2 , and tir $=$ thermal infrared. 
Performance of the gap-filling procedure for the differtent climate variables
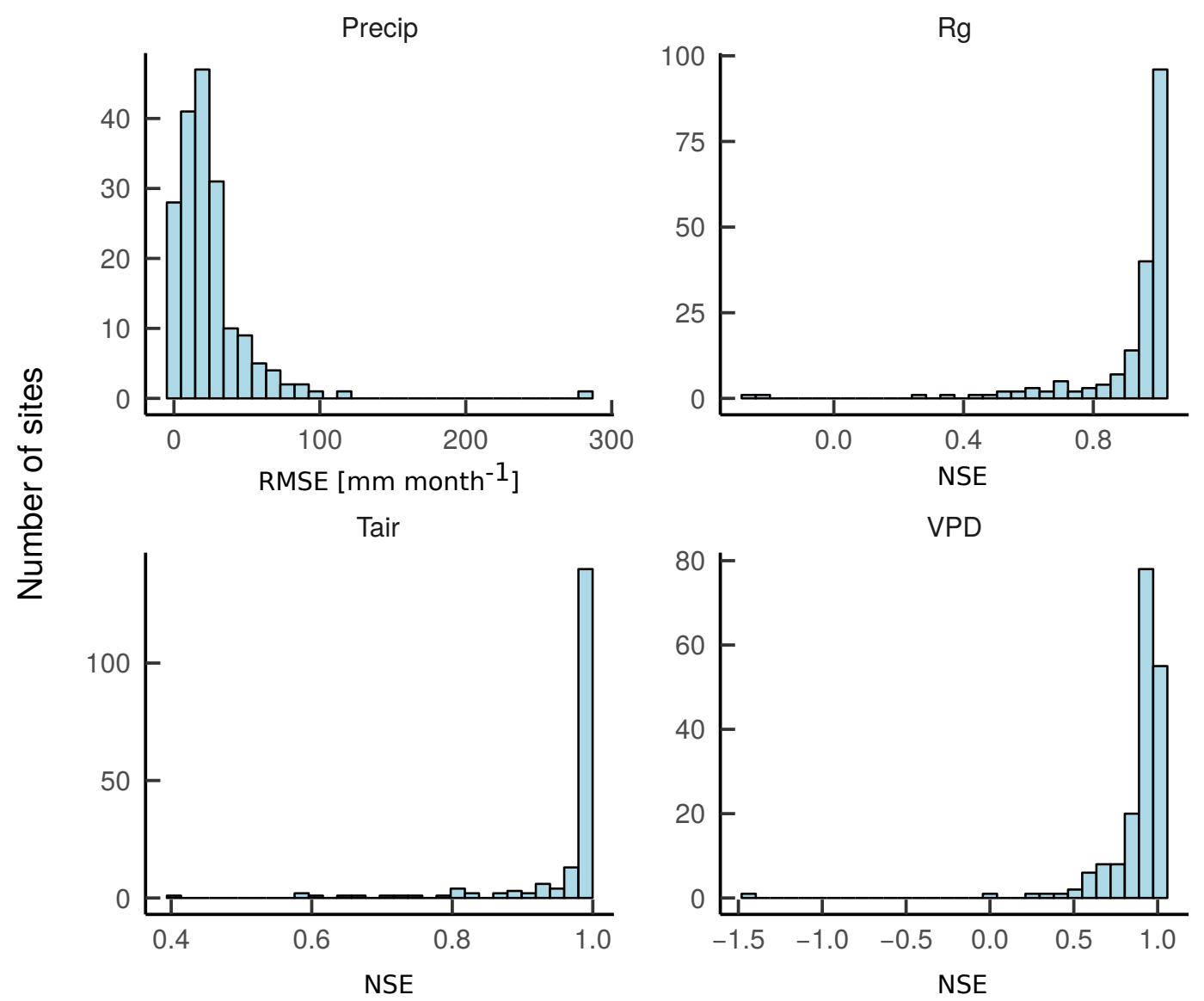

Figure 4.A3: Performance of the gap-filling procedure for the differtent climate variables. Assessment of the gap-filling procedure was done for $\mathrm{T}_{\text {air }}$, Precip, Rg, and VPD. For $\mathrm{T}_{\text {air }}$, Rg, and VPD, the Nash-Sutcliffe efficiency (NSE) is reported, while the root mean squared error (RMSE) is reported for Precip. 
Comparison of the proposed approach against the other model set-ups at site level.

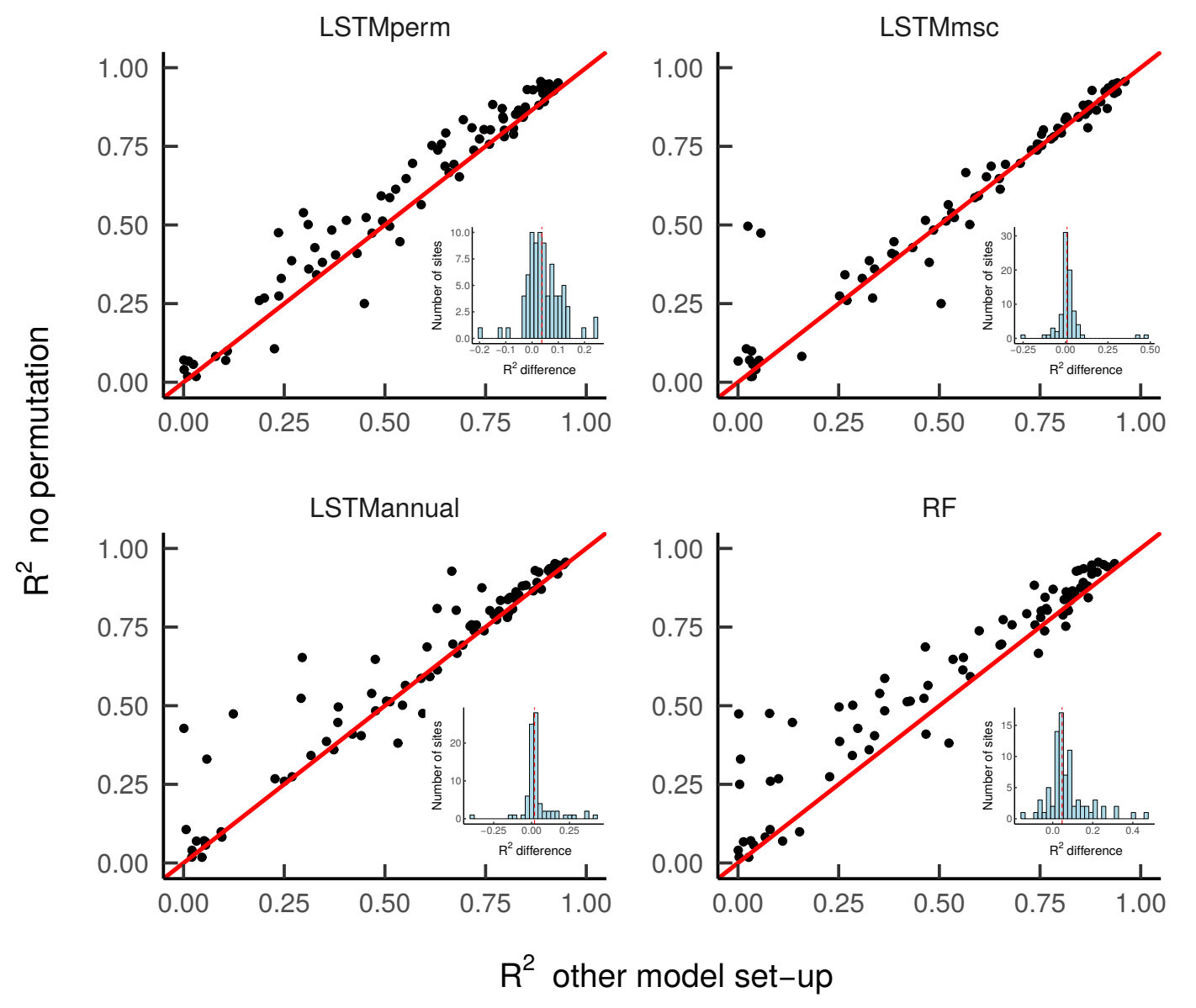

Figure 4.A4: Scatterplots of the coefficient of determination of the proposed approach against the other model set-ups at site level. The coefficient of determination was computed using monthly observed and predicted NEE estimates for each site. Each point represents one site and only the sites with at least one complete year of good quality data $(\mathrm{N}=81$ sites $)$ are shown. 


\section{Chapter 5}

\section{Dynamic and lag effects of climate and vegetation on biosphere-atmosphere $\mathrm{CO}_{2}$ exchange: a global analysis}

This chapter is based on:

S. Besnard, N. Carvalhais, J. G. P. W. Clevers, F. Gans, M. Herold, M. Jung, U. Weber, and M. Reichstein (2019b). "Dynamic and lag effects of climate and vegetation on biosphere-atmosphere $\mathrm{CO}_{2}$ exchange: a global analysis". In preparation 


\begin{abstract}
Climate variability can have both instantaneous and lagged effects on the functioning of terrestrial ecosystems; thereby altering the carbon $(\mathrm{C})$ budgets regionally and globally at different time scales. Yet, the ecological memory (EM) effects associated with past climate \& vegetation conditions on contemporary carbon dioxide $\left(\mathrm{CO}_{2}\right)$ fluxes, including their magnitude and geographic distribution, are poorly understood. Here we present advances in understanding the relevance of eddy-covariance (EC) effects on $\mathrm{CO}_{2}$ fluxes globally from satellite, climate, and eddy-covariance (EC) data for the period 2001-2018. This analysis relies on a dynamic statistical approach; a Long-Short-Term Memory (LSTM) model able to transmit the memory of past vegetation and climate states (or EM effects) to future predictions of $\mathrm{CO}_{2}$ fluxes. EM effects were quantified by computing a difference metric (Root Mean Square Difference, RMSD) between a reference LSTM model with memory to a LSTM model where memory was partly or entirely destroyed via permutation of the time dimension. We found widespread and pronounced EM effects (i.e. high RMSD between the reference and permutation model) on net ecosystem exchange (NEE) across scales. Strong EM effects were observed from dry and boreal climates, as well as from savannas and both deciduous and mixed forests, all with RMSD values ranging from 0.3-0.4 $\mathrm{gC} \mathrm{m}^{-2}$ day $^{-1}$. Globally, limited EM effects were found in water-limited ecosystems, whereas colder and energy-limited regions revealed substantial EM effects on $\mathrm{CO}_{2}$ flux responses. At FLUXNET sites, we found stronger EM effects on NEE than either on ecosystem respiration (ER) or gross primary productivity (GPP). Finally, we also analyzed the 2018 European heatwave and observed that capturing EM effects dampen the net $\mathrm{CO}_{2}$ flux responses to this specific extreme event. These findings emphasize the importance of capturing past vegetation and climate temporal dynamics to better understand the response of contemporary ecosystem $\mathrm{CO}_{2}$ fluxes.
\end{abstract}




\subsection{Introduction}

The structure and function of terrestrial ecosystems are altered by temporal changes in climate and vegetation (Reichstein et al., 2014). As such, the driver-response relationships observed in the functioning of terrestrial ecosystems are not only constant through time, but also respond to both short- and long-term past conditions (Liu et al., 2019; Monger et al., 2015; Montagnani et al., 2018; Seidl et al., 2014). In addition to the instantaneous responses of terrestrial ecosystems to environmental conditions, other responses lag in time due to mortality, changes in plant physiology, and heterotrophic metabolic activity (Frank et al., 2015). Consequently, the temporal vegetation and climate dynamic properties embed fluctuations from seasonal to interannual scales that can exert variable lagged responses on the carbon (C) cycle, depending on both their magnitudes and frequencies (Büntgen et al., 2019). As a result, it is essential to understand the contribution of the climate and vegetation's temporal dynamics to the spatiotemporal patterns of carbon dioxide $\left(\mathrm{CO}_{2}\right)$ fluxes (Thornton et al., 2002; Tramontana et al., 2016; Zscheischler et al., 2017).

While mean seasonal variations of climate and vegetation control most of the spatiotemporal patterns of the terrestrial $\mathrm{CO}_{2}$ fluxes (Besnard et al., 2019a) (Chapter 4), several in-situ studies provide evidence that both past climate conditions and disturbances can significantly influence the contemporary spatiotemporal patterns of the terrestrial $\mathrm{CO}_{2}$ fluxes. For instance, the lag effects of water limitations (Aubinet et al., 2018; Desai, 2014; Liu et al., 2018; Shen et al., 2016) and antecedent air temperature conditions can have either adverse or beneficial effects on vegetation productivity from seasonal to decadal timescales (Buermann et al., 2018; Zhang et al., 2015b; Zielis et al., 2014). Furthermore, climate extreme events (e.g. drought) reduce carbohydrate storage (Frank et al., 2015), which can eventually lead to favorable conditions for other disturbances to occur, such as insect outbreaks or fire (Meigs et al., 2009).

Yet, given the complex and non-linear relationships between past environmental conditions and current vegetation productivity (Bolt et al., 2018; Molen et al., 2011), the effects of antecedent climate and vegetation's temporal dynamics on $\mathrm{CO}_{2}$ fluxes, including their magnitude and geographic distribution, are still poorly understood (Frank et al., 2015; Vicca et al., 2014). Hence, there is a need to investigate the degree to which the recent and past temporal dynamics of both climate and vegetation control the $\mathrm{CO}_{2}$ budgets of terrestrial ecosystems. The complex relation between the vegetation productivity and former environmental conditions highlights the importance of exploiting the vegetation and climate's temporal domain when seeking an understanding of present or future responses of an ecological system (Reichstein et al., 2019). Recently, the application of deep learning (DL) statistical models that translate the response of $\mathrm{CO}_{2}$ fluxes to past climate and vegetation dynamics (i.e. recurrent neural network (RNN)) has shown great potential for understanding how vegetation and climate's temporal dynamics may be used to explain the spatiotemporal variabilities of $\mathrm{CO}_{2}$ fluxes (Besnard et al., 2019a; Reichstein et al., 2018). 
Here, we aim to present advances in understanding the contribution of past climate and vegetation's temporal dynamics (hereafter ecological memory (EM)) (Ogle et al., 2015; Ryo et al., 2019) to $\mathrm{CO}_{2}$ fluxes globally. We use satellite data of greenness, climate data, and eddy-covariance (EC) flux tower data to estimate the contribution of EM to the $\mathrm{CO}_{2}$ fluxes globally for the period of 2001-2018. Our analysis relies on a dynamic statistical approach - a type of RNN called Long-Short-Term Memory (LSTM) - that captures the temporal dependencies of vegetation and climate dynamics. More specifically, we address the questions of (1) what is the magnitude and geographic distribution of EM effects on $\mathrm{CO}_{2}$ fluxes, (2) how do the ecosystem properties and climatic conditions control the observed spatial patterns of EM effects, and (3) how do EM effects contribute to the $\mathrm{CO}_{2}$ flux responses during the 2018 European drought.

\subsection{Material and methods}

\subsubsection{Experimental design}

Following the approach proposed by Besnard et al., 2019a, two LSTM models were trained in order to assess the contribution of vegetation and climate's temporal dynamic properties to the $\mathrm{CO}_{2}$ fluxes globally: (1) a reference LSTM model using the full depth of MODerateresolution Imaging Spectroradiometer (MODIS) time series and in-situ climate data (hereafter no permutation), (2) an LSTM model for which the order of the in-situ predictor-target pairs was randomly permuted (hereafter full permutation) (Fig. 5.1). During the training procedure, 16-day MODIS vegetation indices (VIs) (i.e. normalised difference vegetation index (NDVI) (Tucker, 1979) (equation 5.2) and enhanced vegetation index (EVI) (Huete et al., 2002) (equation 5.3)) and climate (i.e. air temperature $\left(\mathrm{T}_{\text {air }}\right)$, total precipitation (Precip), global radiation $(\mathrm{Rg})$, and vapor pressure deficit (VPD)) time series were used as input data, while 16-day $\mathrm{CO}_{2}$ flux data (net ecosystem exchange (NEE), gross primary productivity (GPP), and ecosystem respiration (ER)) were the response variables. Due to the random initialization of the LSTM models, 15 runs of the two aforementioned LSTM model set-ups were performed. The comparison between the no permutation and the full permutation model outputs provided insights on the contribution EM effects to the $\mathrm{CO}_{2}$ fluxes with regard to both the seasonal cycle and the interannual variability. The importance of EM effects was analyzed at the global scale by translating gridded inputs of 16-day $\mathrm{T}_{\text {air }}$, total Precip, Rg, VPD, NDVI, and EVI into 15 global time-varying $0.05^{\circ}$ grids of NEE for the 2001-2018 period. The median of the ensemble for each pixel and time step of these 15 spatiotemporal grids was further used in the analysis. The construction of the different spatiotemporal grids of $\mathrm{CO}_{2}$ fluxes was done within the Earth System Data Cube platform (https://www . earthsystemdatalab.net/).

Additionally, the no permutation model was compared to a third model at FLUXNET site level only: (3) an LSTM model for which the order of each 16-day observation was randomly permuted across the years, but the annual cycle was kept unpermuted (hereafter permuted year) 
(Fig. 5.1). Contrasting no permutation against permuted year enabled us to assess the relevance of previous years to the current $\mathrm{CO}_{2}$ flux responses (Table 5.1). At FLUXNET site level, an assessment of the no permutation, full permutation, and permuted year models was provided not only for NEE but also for GPP and ER.

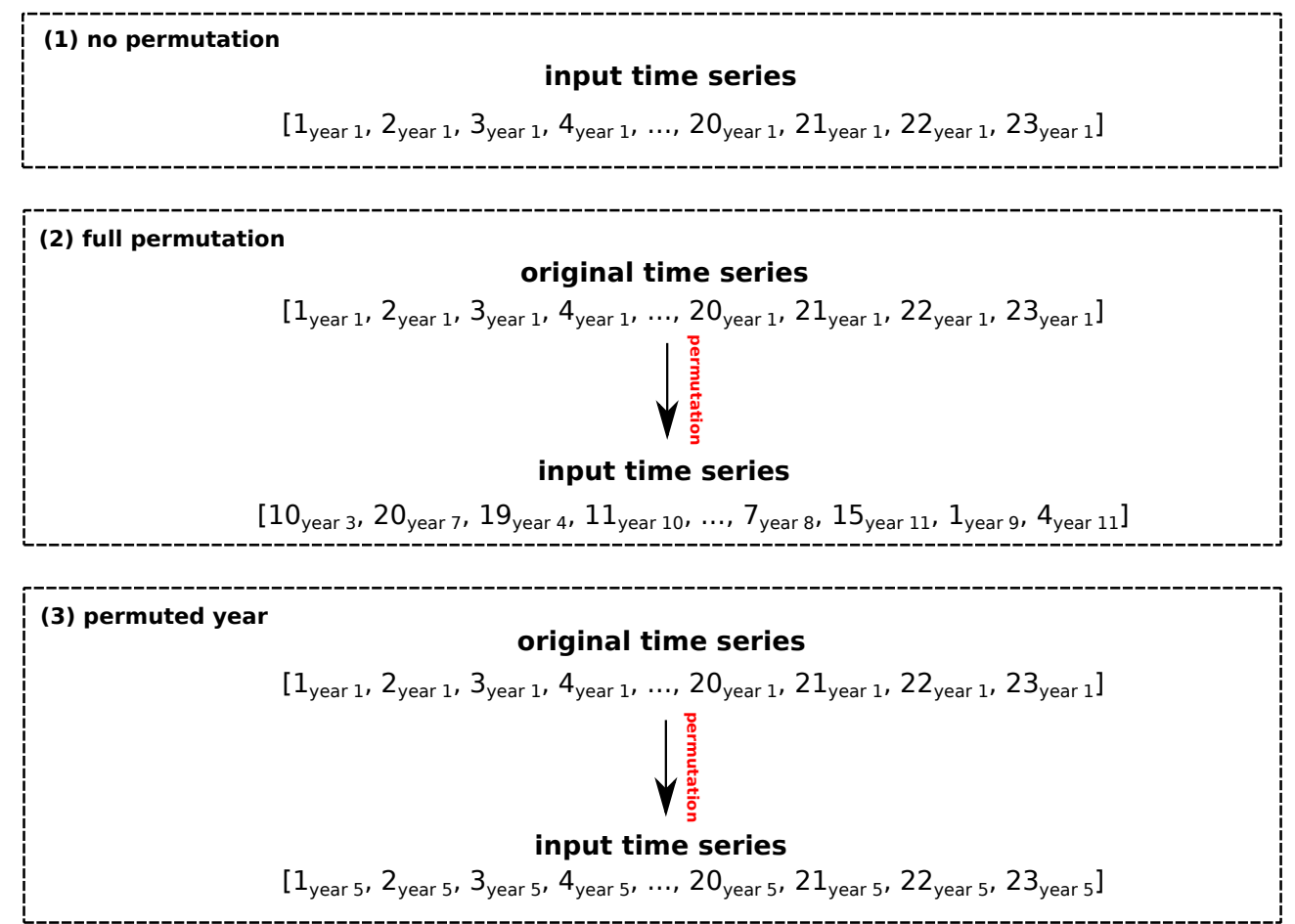

Figure 5.1: Illustration of the different temporally permuted order of the predictor-target pairs. This example represents the different permutation scenarios for an individual year. The numbers from one to 23 represent each 16-day observations of the year while the subscript notations are the number of the years. The presented temporal permutations were performed for the $\mathrm{n}$ years during the training procedure of each LSTM model.

Table 5.1: Design of the factorial experiment. $X$ means that the variant was used to study the respective topic of each row.

\begin{tabular}{l|ccc} 
& no permutation & full permutation & permuted year \\
\hline Full temporal context & $\mathbf{X}$ & $\mathbf{X}$ & \\
Interannual variability & $\mathbf{X}$ & & $\mathbf{X}$
\end{tabular}

Comparing the outputs of the different LSTM models (Table 5.1) at gridcell levels was done using the root mean square difference (RMSD) (equation 5.1):

$$
R M S D=\sqrt{\frac{\sum_{i=1}^{n}\left(\text { model } X_{i}-\operatorname{model} Y_{i}\right)^{2}}{n}}
$$

where $\mathrm{X}$ means permutation and $\mathrm{Y}$ no permutation. EM effects were considered limited when we found low RMSD estimates and vice-versa. 


\subsubsection{Site level data}

\section{Eddy-covariance data}

The current dataset consisted of 346 sites belonging to either the version 2 of the LaThuile FLUXNET or the FLUXNET2015 datasets (https://fluxnet.fluxdata.org) of the FLUXNET global network (Baldocchi, 2008; Baldocchi et al., 2001). Nine plant functional types (PFTs) were considered: cropland (CRO) $(\mathrm{N}=40)$, deciduous broadleaf forest (DBF) $(\mathrm{N}=42)$, evergreen broadleaf forest $(\mathrm{EBF})(\mathrm{N}=28)$, evergreen needleleaf forest $(\mathrm{ENF})(\mathrm{N}=81)$, grassland (GRA) ( $=63)$, mixed forest (MF) $(\mathrm{N}=14)$, savanna (SAV) $(\mathrm{N}=19)$, shrubland $(\mathrm{SH})(\mathrm{N}=24)$, and wetland (WET) $(\mathrm{N}=35)$. For each site, we obtained continuously measured or gap-filled of NEE (variable names are NEE_f and NEE_VUT_USTAR50 for LaThuile and FLUXNET2015 datasets, respectively), GPP (variable names are GPP $f$ and GPP_NT_VUT_USTAR50 for LaThuile and FLUXNET2015 datasets, respectively), and ER (variable names are Reco and RECO_NT_VUT_USTAR50 for LaThuile and FLUXNET2015 datasets, respectively) at halfhourly time intervals. We used GPP and ER estimates derived from the nighttime partitioning method (Reichstein et al., 2005). The half-hourly NEE, GPP, and ER observations were aggregated into 16-day averages. Only 16-day observations with more than $80 \%$ of the original or good quality gap-filled data were considered in this analysis (based on the variables NEE fqcOK and NEE_VUT_USTAR50_QC for LaThuile and FLUXNET2015 datasets, respectively), otherwise observations were discarded.

\section{Remote sensing data}

For each FLUXNET site, the entire multi-temporal 16-day MODIS collection at 250m spatial resolution (MOD13Q1 version 6) was downloaded from the Google Earth Engine (GEE) platform (Gorelick et al., 2017) (https : //earthengine.google.com/). The NDVI (equation 5.2) and the EVI were extracted (equation 5.3).

$$
\begin{gathered}
N D V I=\frac{(N I R-R E D)}{(N I R+R E D)} \\
E V I=2.5 \cdot \frac{(N I R-R E D)}{(N I R+6 \cdot R E D-7.5 \cdot B L U E+1)}
\end{gathered}
$$

where NIR, RED, and BLUE denote surface reflectances in the wavelengths regions of $858 \mathrm{~nm}$, $645 \mathrm{~nm}$, and $469 \mathrm{~nm}$, respectively.

A circular buffer of $1 \mathrm{~km}$ radius centered around each FLUXNET tower was defined to better represent the area of the $\mathrm{CO}_{2}$ flux footprints. The quality reliability of VI pixel layer (i.e. SummaryQA layer) of the MOD13Q1 version 6 product was used for filtering out low quality observations within the cutout (i.e. cloudy pixels and pixels covered with snow/ice were 
discarded). A mean value of the good quality pixels within the different MODIS cutouts was extracted for each MODIS VI. Time series of MODIS VI were gap-filled to create continuous 16-day time series by predicting each MODIS VI with a Random Forest (RF) model (Breiman, 2001; Kuhn, 2008) using climate variables (i.e. $\mathrm{T}_{\text {air }}, \mathrm{Rg}$, and potential global radiation $\left(\mathrm{R}_{\text {pot }}\right)$ ) as predictive variables (Fig. 5.A1).

\section{Climate data}

Local climatic variables (i.e. $\mathrm{T}_{\text {air }}$, total Precip, $\mathrm{Rg}$, and VPD) were obtained at site level from the FLUXNET dataset. These climate variables were further aggregated to 16-day and gapfilled with respective down-scaled time series to provide climate data covering the entire MODIS era. The downscaling procedure of each climate variable was done using the CRUNCEP datasets (Viovy, 2018) for the period of 1901-2015. At the FLUXNET site level, the time series of the three nearest gridcells of each climate variable (i.e. $\mathrm{T}_{\text {air }}$, total Precip, Rg, and VPD) from the CRUNCEP datasets (Viovy, 2018) were extracted for training several statistical models (i.e. relational logistic regression, kernel ridge regression, Gaussian processes regression, and neural networks). The best statistical model for each site was consequently selected based on the highest Nash-Sutcliffe model efficiency (NSE) in order to obtain predicted climate variables at the site level (Fig. 5.A2). These predicted time series were further used for gap-filling the in-situ climate variables (Besnard et al., 2019a).

\subsubsection{Global gridded products}

The global NDVI and EVI Climate Modeling Grid MOD13C1 Version 6 products (Didan, 2015) (https://lpdaac.usgs.gov/products/mod13c1v006/) at $0.05^{\circ}$ spatial resolution and 16day temporal resolution for the 2001-2018 period were used to retrieve information on land surface vegetation at the global scale. Assuming that there is no vegetation activity during the winter observations in the Northern latitude $\left(>60^{\circ} \mathrm{N}\right)$, a linear interpolation on both the NDVI and EVI mean seasonal cycles between neighboring observations of each gridcell was performed in order to gap-fill the missing observations of the winter period in the original NDVI and EVI MOD13C1 Version 6 products. The other gaps in the gridded MODIS products related to cloudy data were filled by calculating the average of good data from all previous years of a specific composite period (Didan, 2015).

Gridded climate products of $\mathrm{T}_{\text {air }}$, total Precip, $\mathrm{Rg}$, air relative humidity (q), and air pressure (P) were obtained from the ERA5 datasets (https://apps.ecmwf.int/datasets/licences/copernicus/) at $0.5^{\circ}$ spatial resolution and half-hourly temporal resolution. $\mathrm{T}_{\text {air }}, \mathrm{q}$, and $\mathrm{P}$ products were used to calculate VPD according to the first principles of Monteith and Unsworth (2007). Climate gridded products were nearest neighbor resampled to $0.05^{\circ}$ and aggregated to 16-day temporal resolution in order to match the spatial and temporal resolutions of the MOD13C1 version 6 
global products. A summary of all the gridded products considered in the analysis can be found in Table 5.2.

Table 5.2: A summary of the datasets used in this study. Documentation about the characteristics of the ERA5 products can be found on the European Centre for Medium-Range Weather Forecasts website (https://apps.ecmwf.int/datasets/licences/copernicus/).

\begin{tabular}{|c|c|c|c|c|c|}
\hline Variable & Product & Native spatial resolution & Native temporal resolution & Spanning period & Source \\
\hline Normalized difference vegetation index & MOD13C1 v6 & $0.05^{\circ}$ & 16-day & 2001-2018 & Didan, 2015 \\
\hline Enhanced vegetation index & MOD13C1 v6 & $0.05^{\circ}$ & 16-day & 2001-2018 & Didan, 2015 \\
\hline Air temperature & ERA5 & $0.5^{\circ}$ & half-hourly & 1979-2018 & - \\
\hline Total precipitation & ERA5 & $0.5^{\circ}$ & half-hourly & 1979-2018 & - \\
\hline Global radiation & ERA5 & $0.5^{\circ}$ & half-hourly & $1979-2018$ & - \\
\hline Air relative humidity & ERA5 & $0.5^{\circ}$ & half-hourly & 1979-2018 & - \\
\hline Air pressure & ERA5 & $0.5^{\circ}$ & half-hourly & $1979-2018$ & - \\
\hline
\end{tabular}

\subsection{Results and discussion}

\subsubsection{EM effects on net $\mathrm{CO}_{2}$ exchange at the global scale}

Overall, there were widespread and substantial EM effects for NEE at the global scale (Fig. 5.2a). Substantial EM effects were apparent in the seasonally dry climates (e.g. central Europe), tropical savannas (e.g. India, northern Australia, southern Latin America), semi-arid/monsoonal regions (e.g. the Sahel, part of the dry tropics, and eastern Australia), agricultural/managed areas (e.g. eastern United States and western Europe), northeastern Eurasia, part of Siberia, and eastern temperate Asia. However, wet tropical regions depicted limited EM on the net $\mathrm{CO}_{2}$ fluxes. Temporal variations of the no permutation and full permutation models in selected locations (Fig. 5.A3) provided insights in explaining the aforementioned spatial patterns of EM. For instance, in eastern United States, the full permutation model had substantial discrepancies with the no permutation model when modeling the seasonal cycle of the net $\mathrm{CO}_{2}$ fluxes, in particular at the peak of the growing season where lower NEE estimates were observed in the full permutation model. Similar temporal patterns were observed in the other selected regions (e.g. the Sahel, western Europe, Eurasia, dry tropics in Africa), while in regions such as India there were substantial differences between the full permutation and no permutation models at the start and the end of the growing season. Interestingly, comparing the RMSD estimates against interannual variability in the $\mathrm{CO}_{2}$ fluxes (Fig. 5.2b) suggested that the regions with the highest EM effects (i.e. highest RMSD estimates) had a rather moderate interannual variability. Some of the regions with high NEE interannual variability (e.g. Miombo woodlands, eastern Australia grasslands) depicted relatively lower EM effects. This may suggest that ecosystems in such regions have adapted with an array of morphological, physiological, and biochemical conditions (Bohnert et al., 1995) to endure important climate variabilities. Yet, we found that there were also regions with high NEE interannual variability (e.g. dry tropics in south America) that had substantial EM effects. Therefore, regions depicting strong NEE interannual variability that are usually located 
in arid to semi-arid regions, known to be susceptible to variations in moisture supply (Jung et al., 2011), appeared to have variable sensitivity to climate and vegetation variabilities (Fig. 5.2d). Because semi-arid and arid regions are expected to experience an increase in frequency and severity of climate extreme events (Giorgi, 2006; Hoerling et al., 2011; Sheffield and Wood, 2008), the apparent contribution of EM effects might be amplified in the near future for such ecosystems.

(a)

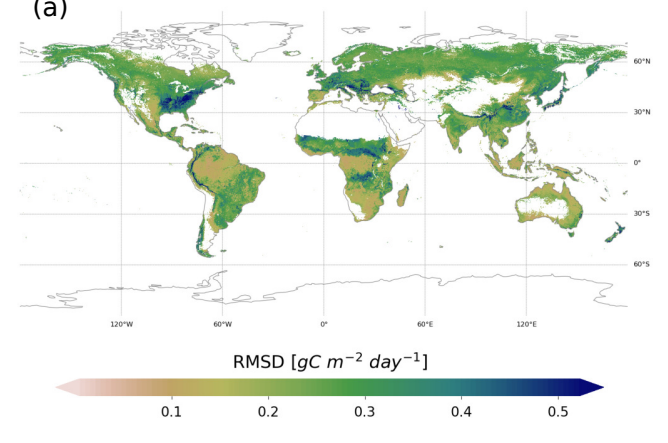

(c)

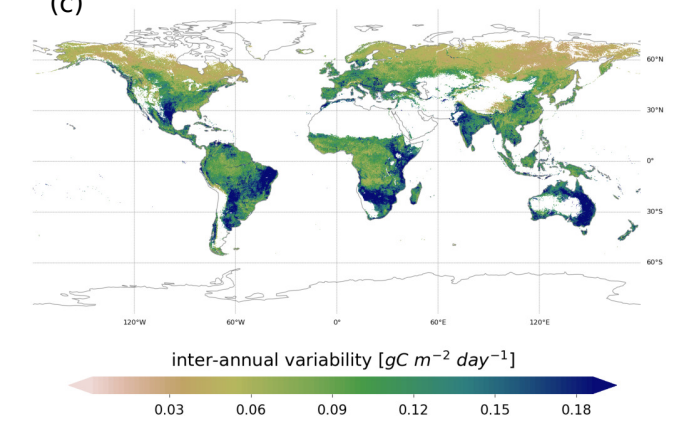

(b)

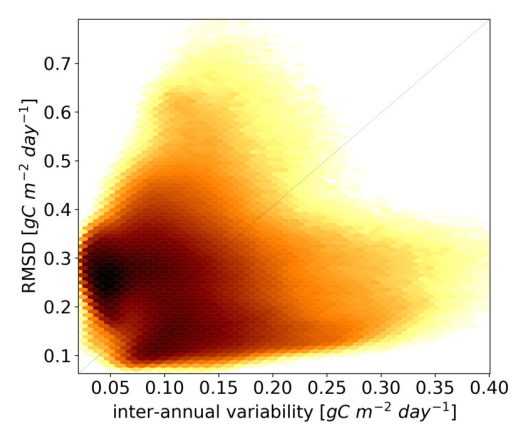

(d)

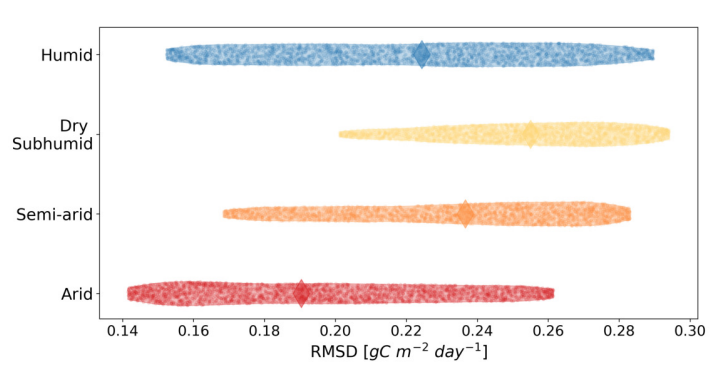

Figure 5.2: Spatial patterns of RMSD estimates between no permutation vs. full permutation models in NEE (a). Regions with GPP estimates lower than $200 \mathrm{gC} \mathrm{year}^{-1}$ were masked out. RMSD estimates are also contrasted against the interannual variability in NEE derived from the no permutation product (b). In (c), the spatial patterns of NEE interannual variability for the no permutation model are shown. Interannual variability was computed as the standard deviation of the annual estimates derived from the no permutation product. In (d), violin plots represent the RMSD estimates (truncated to the $25^{\text {th }}$ and $75^{\text {th }}$ percentiles) across classes of an aridity index. The latter was derived from the ratio of total Precip and potential evapotranspiration (PET). Arid: $0.05>$ Precip/PET $<=0.2$, Semi-arid: $0.2>$ Precip/PET $<=0.5$, Dry Subhumid: $0.5>$ Precip/PET $<=0.65$, and Humid: $0.65>$ Precip/PET.

The observed patterns of EM effects on the $\mathrm{CO}_{2}$ fluxes suggested they were not only ecosystem, but also varied with climate (Fig. 5.3). The EM effects on NEE prevailed in forested ecosystems (i.e. deciduous broadleaf, deciduous needleleaf, evergreen needleleaf, and mixed forests) with RMSD estimates ranging from $0.25-0.40 \mathrm{gC} \mathrm{m}^{-2} \mathrm{day}^{-1}$, while evergreen broadleaf forests ( $\mathrm{RMSD}=0.15 \pm 0.069 \mathrm{gC} \mathrm{m}^{-2} \mathrm{day}^{-1}$ ) exhibited relatively low EM effects (Fig. 5.3a). Savannas ( $\mathrm{RMSD}=0.26 \pm 0.031 \mathrm{gC} \mathrm{m}^{-2} \mathrm{day}^{-1}$ ), croplands (RMSD $=0.25 \pm 0.024 \mathrm{gC} \mathrm{m}^{-2} \mathrm{day}^{-1}$ ), and shrublands $\left(\mathrm{RMSD}=0.21 \pm 0.026 \mathrm{gC} \mathrm{m}^{-2} \mathrm{day}^{-1}\right.$ ) had rather moderate $\mathrm{EM}$ effects for NEE. 
In addition, we found that overall the highest contribution of EM effects was located in dry climate zones $\left(\mathrm{RMSD}=0.26 \pm 0.019 \mathrm{gC} \mathrm{m}^{-2} \mathrm{day}^{-1}\right.$ ) and boreal $\left(\mathrm{RMSD}=0.26 \pm 0.037 \mathrm{gC} \mathrm{m}^{-2}\right.$ day $^{-1}$ ) climates, followed by temperate $\left(\mathrm{RMSD}=0.25 \pm 0.051 \mathrm{gC} \mathrm{m}^{-2} \mathrm{day}^{-1}\right.$ ), and tropical ( $\mathrm{RMSD}=0.20 \pm 0.041 \mathrm{gC} \mathrm{m}^{-2} \mathrm{day}^{-1}$ ) regions (Fig. 5.3b). It has been shown that EM effects can control vegetation productivity in semi-arid regions of the boreal belt (Seddon et al., 2016). Additionally, several studies have provided evidence for lagged effects of spring warmth on plant productivity during the subsequent summer and autumn periods in the northern high latitudes (Buermann et al., 2018; Menzel et al., 2006), which could explain the strong EM observed in deciduous temperate-boreal ecozones. Nevertheless, there was a large spread of the RMSD estimates within the same ecosystem types or climatic biomes, suggesting that areas having similar ecosystem properties or belonging to the same climatic regime could respond quite differently to past dynamics of vegetation and climate. These results suggest that $\mathrm{CO}_{2}$ flux responses to antecedent environmental conditions are not necessarily ecosystem or climate specific, but also depend quite often on the local environmental conditions.

(a)

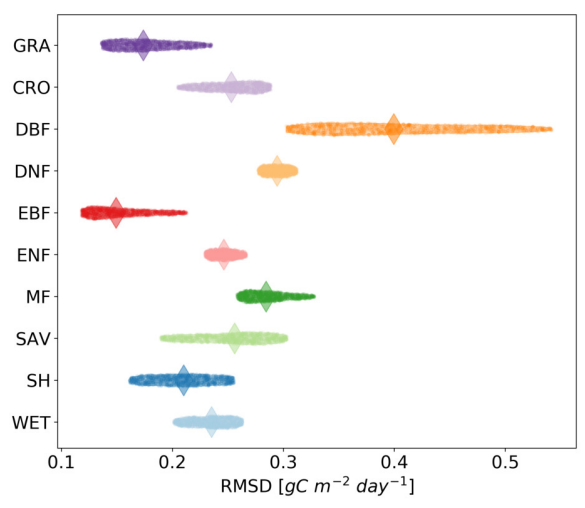

(b)

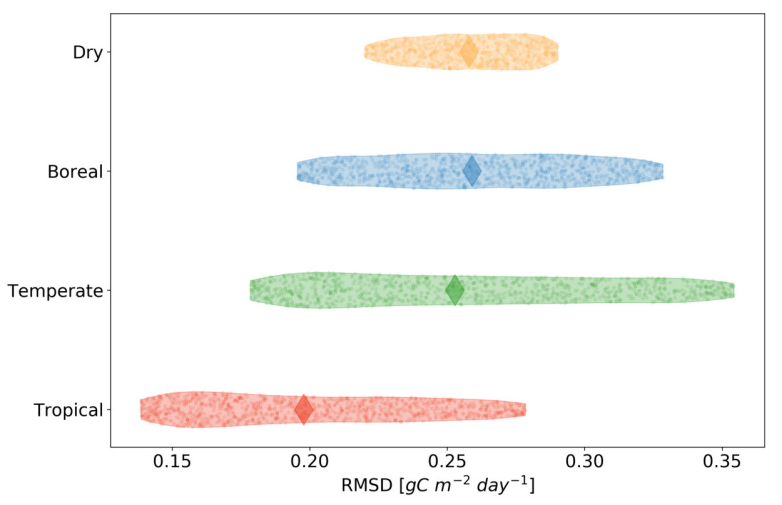

Figure 5.3: RMSD estimates between no permutation vs. full permutation models) in NEE among PFTs (a) and climatic regions (b) for NEE. Violin plots represent the RMSD estimates (truncated to the $25^{\text {th }}$ and $75^{\text {th }}$ percentiles) across each PFT and climatic region. PFT classes were derived from the MCD12Q1 MODIS product (Friedl et al., 2010) while the climatic regions were derived from the Köppen Geiger A2 Scenario 2001-2025.

By computing RMSD estimates between the no permutation and full permutation models for different temporal scales (i.e. seasonal cycle, seasonal anomalies, interannual, and mean fluxes), we were able to assess the relevance of EM across scales (Fig. 5.4). While a large part of the study area exhibited strong EM effects on the seasonal cycle of NEE (Fig. 5.4a), some regions also had substantial EM effects on the seasonal anomalies of NEE (Fig. 5.4b). For instance, regions with less seasonal variations (e.g. wet rainforest in the Amazon region, the Congo basin, southeast Asia) had strong EM effects on the seasonal anomalies of NEE. Climate extreme events, such as the El Niño that imposed extreme warming and dry conditions, can have instantaneous and substantial impacts on the seasonal anomalies of the $\mathrm{CO}_{2}$ fluxes in the tropics (Bastos et al., 2018) but these climate effects can also impact the $\mathrm{CO}_{2}$ fluxes during the 
subsequent months after the event occurred (Kim et al., 2016). Some regions depicted strong EM effects both at the seasonal scale (Fig. 5.4a) and on the mean fluxes (Fig. 5.4c) for NEE (e.g. Miombo, Sahel, Eurasia, semi-arid northern Australia, India). On the other hand, regions being strongly managed (e.g. western Europe, the Corn Belt, southeast of China) appeared to have low EM effects on the mean net $\mathrm{CO}_{2}$ fluxes. These findings possibly suggested that most of the observed EM effects at the seasonal scale in such managed regions may be related to differences in the timing of the growing season's onset (Myneni et al., 1997) between the no permutation and full permutation experiments rather than differences in the magnitude of the growing season; therefore the mean $\mathrm{CO}_{2}$ fluxes appeared to be rather similar between the two experiments. However, we found that the sign, the magnitude, and the timing of the growing season were different between the no permutation and full permutation models (Fig. 5.A4). Only a small fraction of the global vegetated land area depicted substantial EM effects both on the overall time series and at interannual scales (Fig. 5.4d). These areas with dual EM effects were located in water-limited systems (e.g. Miombo, eastern Australia, eastern Africa, midwest of the United States) (Fig. 5.2c). EM effects were also apparent at the interannual scale for some parts of the wet tropics that could be related to fire regimes in the Amazon (Aragão et al., 2018) and deforestation in South-East Asia (Zeng et al., 2018), even though relatively low EM effects were found in the overall time series (Fig. 5.2a).

The geographical distribution of EM effects on $\mathrm{CO}_{2}$ fluxes (Fig. 5.2) suggested that the spatial variability of biospheric and atmospheric variables could largely explain these patterns. The global relationships between the EM effects (i.e. RMSD estimates) and biospheric and atmospheric variables (Fig. 5.5) revealed that colder regions exhibited stronger EM effects on NEE than warmer regions (Fig. 5.5a). We found that there was a decrease in EM effects with an increase in global radiation (Fig. 5.5c), suggesting that capturing EM effects is important when examining NEE changes in energy limited regions. These regions present a moderate to high percentage of tree cover as well as a large fraction of deciduous and mixed forests across the globe. Additionally, water-limited regions (low precipitation and high VPD) (Fig. 5.5b and d) with relatively low NDVI (e.g. savannas, shrublands, desert, and semi-arid regions) (Fig. 5.5e), had low EM effects. Such regions with limited soil water availability typically have low/sparse tree cover with a high fraction of grass. As such, photosynthesis and respiratory fluxes, hence NEE variability, are mostly controlled by instantaneous environmental conditions (e.g. rainfall, low intensity fire), thereby EM effects were less obvious in these regions as this is just part of the normal environmental variability. Finally, EM effects appeared to increase from regions with low $\mathrm{C}$ stocks to regions with moderate $\mathrm{C}$ stocks, while areas with high aboveground biomass (AGB) depicted lower EM effects (Fig. 5.5f). 
(a)

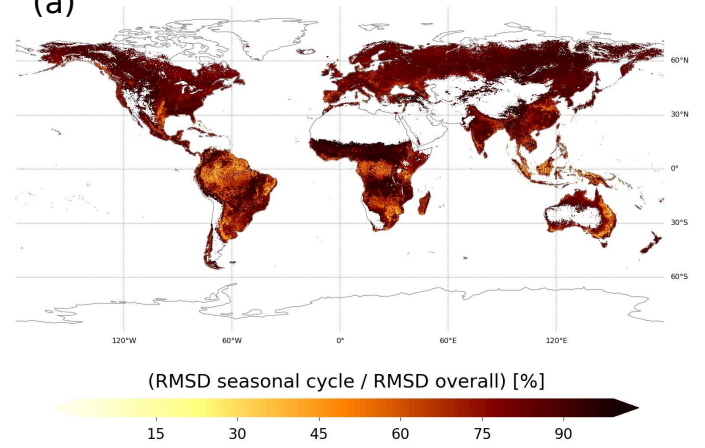

(c)

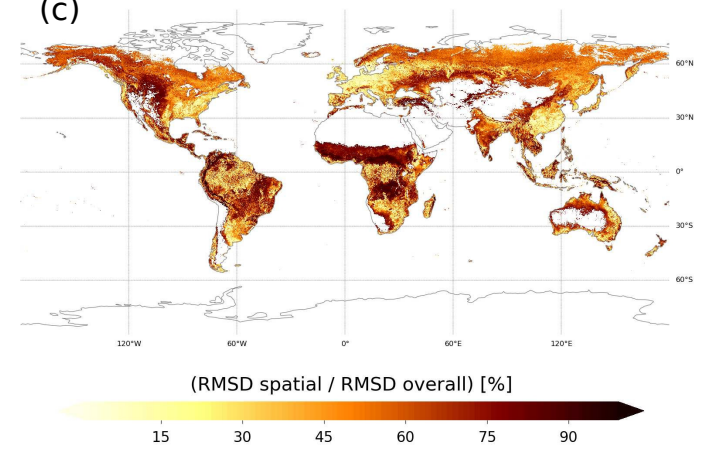

(b)

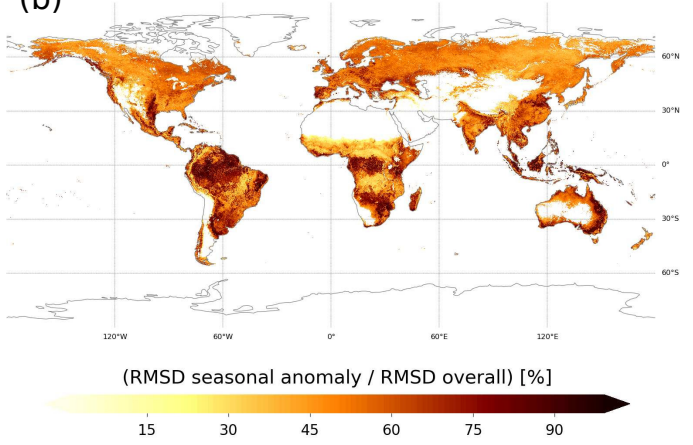

(d)

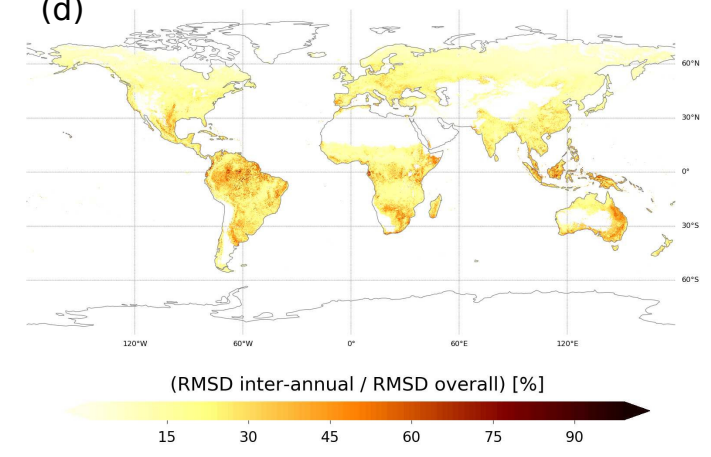

Figure 5.4: Spatial patterns of normalized RMSD estimates between no permutation and full permutation models across scales (i.e. seasonal cycle (a), seasonal anomalies (b), mean fluxes (c), and interannual (d)). The maps show the ratio between the RMSD estimates for different scales (i.e. seasonal cycle, seasonal anomalies, interannual or mean fluxes) and the RMSD esimtates estimated on the entire time series (i.e. overall). The differences are expressed in percentage (\%).

\subsubsection{The implications of EM effects on net $\mathrm{CO}_{2}$ fluxes during the 2018 European heat- wave}

The relevance of EM effects was explored for the 2018 European heatwave for NEE (Fig. 5.6). First of all, we found both widespread positive (i.e. less $\mathrm{C}$ uptake in 2018) and negative (i.e. more $\mathrm{C}$ uptake in 2018) anomalies over Europe in 2018 regardless whether past vegetation and climate dynamics were being considered or not (i.e. full permutation and no permutation) (Fig. 5.6a). Negative impacts of drought on NEE were mainly apparent in western Europe while the Mediterranean region and most of eastern Europe were not affected by the heatwave (i.e. negative anomalies or no anomaly). We found that the anomalies observed in NEE were non-linearly related with the anomalies in total Precip (Fig. 5.A5d) but not coupled with air temperature anomalies (Fig. 5.A5b). As such, regions having a relatively low amount of total Precip during 2018 compared to the period 2001-2017 (except 2003 due to the heatwave and drought during the year 2003 (Ciais et al., 2005)) depicted a lower C uptake. The fact that NEE anomalies were more related to the anomalies in precipitation may suggest that terrestrial ecosystems responded mainly to rainfall deficit rather than to heat, which appeared to be an opposite pattern 
(a)

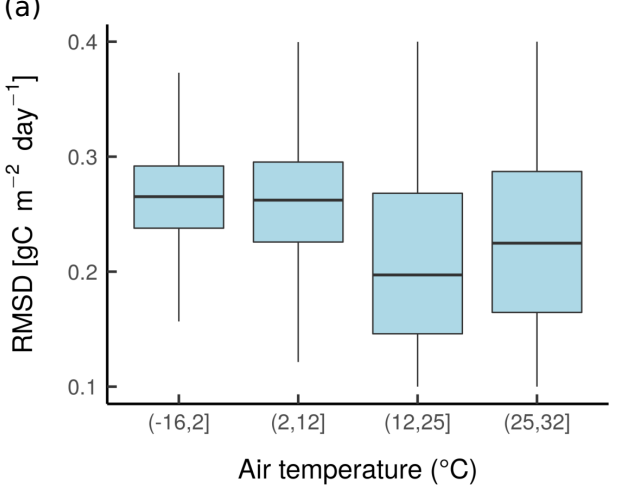

(c)

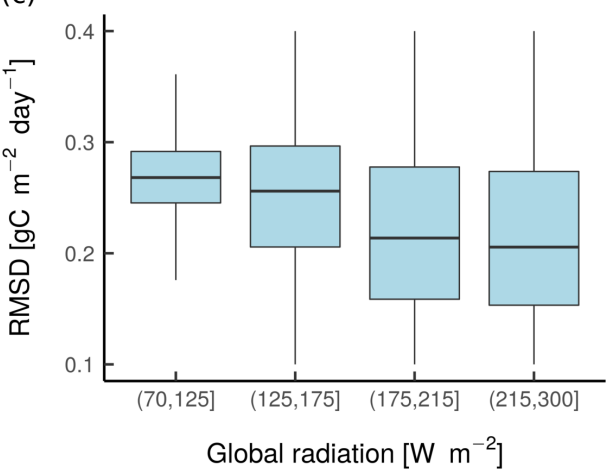

(e)

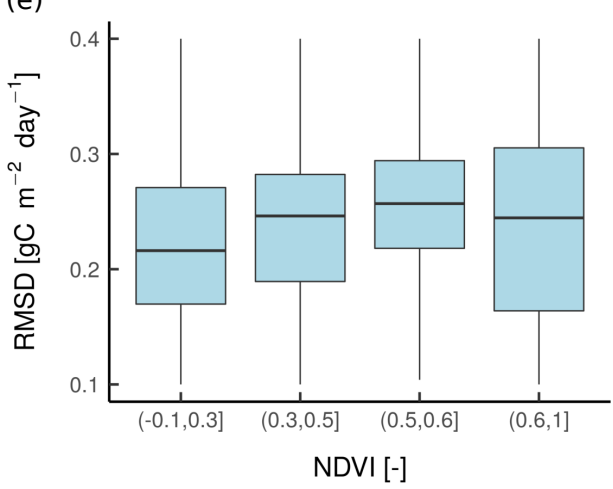

(b)

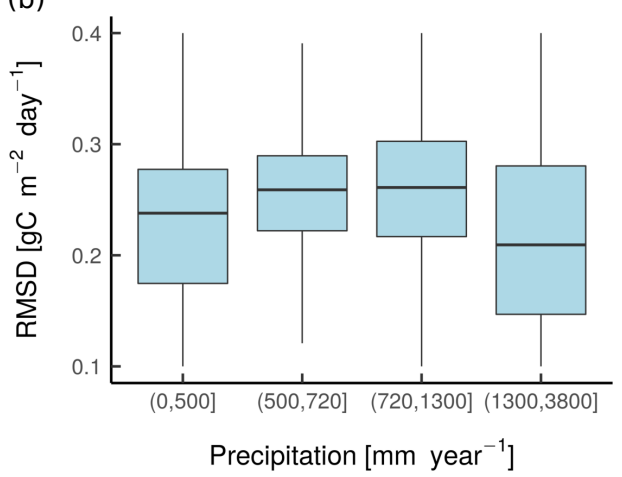

(d)

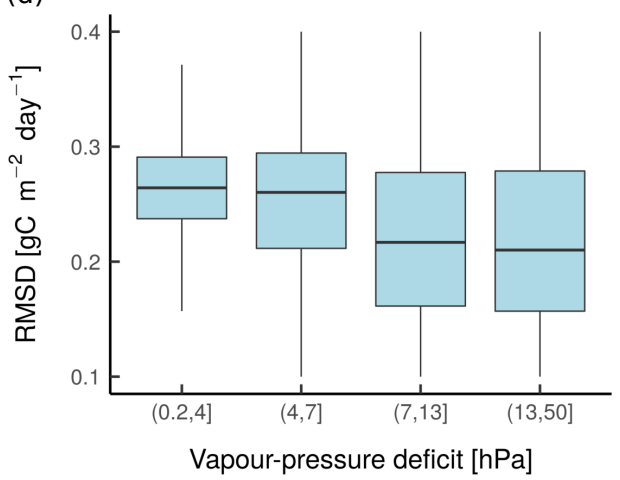

(f)

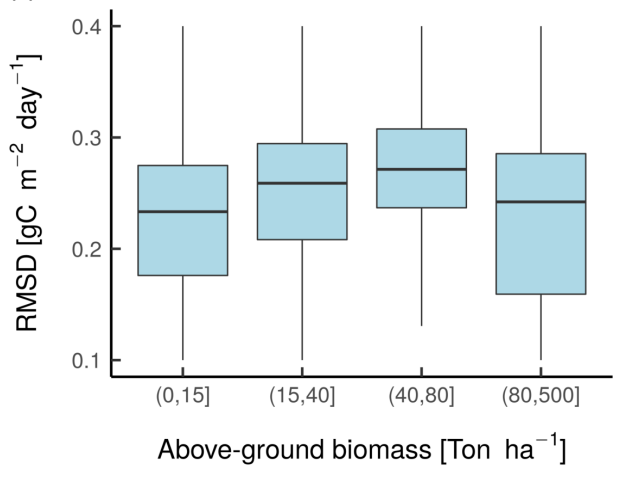

Figure 5.5: Boxplots of global relationships of RMSD estimates between no permutation and full permutation models and a series of atmosphere and biosphere variables. Comparison of the spatial patterns between RMSD estimates and atmosphere or biosphere variables are shown. Atmosphere category of predictors includes air temperature, precipitation, global radiation, and vapor pressure deficit (Dee et al., 2011), while biosphere category of predictors includes normalized vegetation index (Didan, 2015) and above-ground biomass (Globbiomass project, http://globbiomass.org/). Box-plots represent the RSMD mean \pm std across classes for each atmosphere or biosphere variable.

to the one observed during the 2010 heatwave in Russia (Bastos et al., 2014). The fact that the observed NEE anomalies were negatively correlated with the anomalies in NDVI (Fig. 5.A5f) suggested that the decrease of net $\mathrm{C}$ uptake observed during the 2018 European drought can be attributed to reduced photosynthetic activity. Overall, it seemed that the magnitude of the 
anomalies (either positive or negative) were higher for the full permutation model. The difference in anomaly between the full permutation and no permutation maps (i.e. top right map in Fig. 5.6a) revealed that the contribution of EM effects was variable over Europe in terms of magnitude and sign, resulting in either a higher $\mathrm{C}$ uptake (i.e. blue color) or a lower $\mathrm{C}$ uptake (i.e. red color). Surprisingly, we found that EM effects dampened positive or negative anomalies (Fig. 5.6b). It was also interesting to see that the differences in anomalies between the no permutation and full permutation were sensitive to tree cover as regions with low tree cover $(0-20 \%$ and $20-40 \%)$ had higher spread in anomalies differences than regions with high tree cover $(>40 \%)$ (Fig. 5.6c). The lower variability in regions with tree cover $>40 \%$ may indicate an inherent stability of forest systems with relatively high tree cover to severe heat stress. Future releases of the FLUXNET dataset will not doubt help to not only benchmark the magnitude and the sign of $\mathrm{CO}_{2}$ flux anomalies such as these, but also to corroborate our findings related to EM effects and the 2018 European heatwave.

(a)
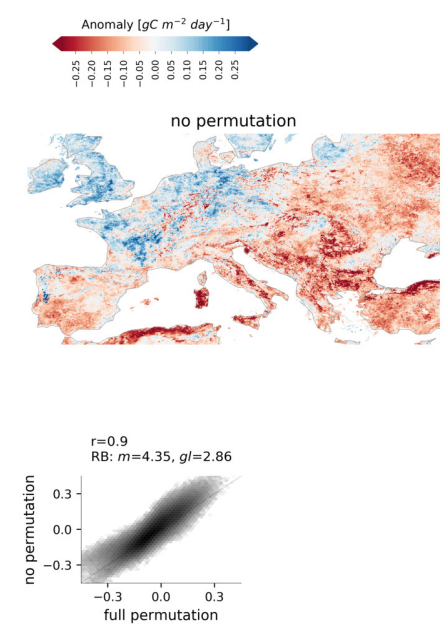

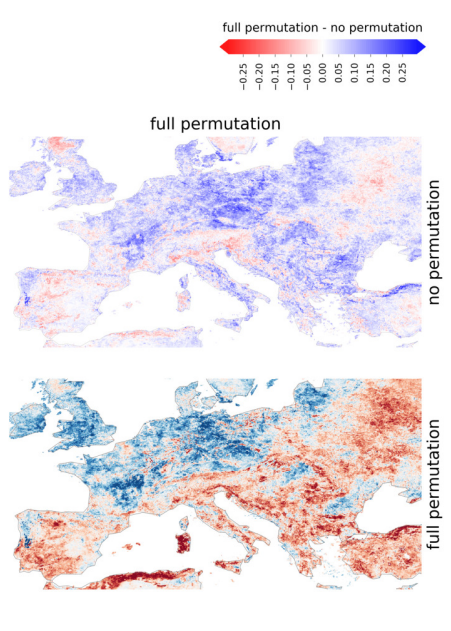

(c) (b)
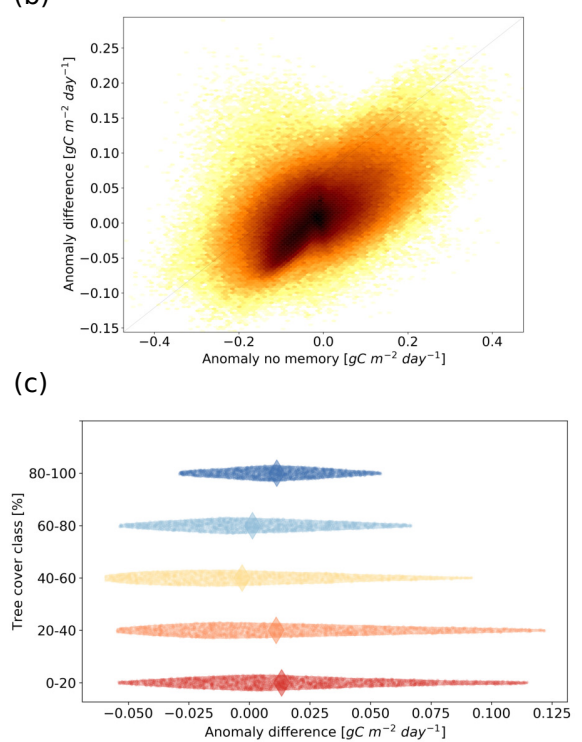

Figure 5.6: Comparison of the annual anomalies derived from the no permutation and the full permutation models (a) during the European 2018 heatwave. The maps show the annual anomalies in net ecosystem exchange (NEE) for the year 2018. Anomalies were computed by calculating the difference between annual NEE in 2018 and mean annual NEE over the period 2001-2017 (except 2003). A difference map between the calculated anomalies for the full permutation and the no permutation experiments (i.e. full permutation anomaly - no permutation anomaly) is also shown. (b) The differences in anomalies (i.e. full permutation anomaly - no permutation anomaly) are also plotted against the anomalies reported in the full permutation experiment and (c) tree cover classes (Hansen et al., 2013). 


\subsubsection{EM effects on $\mathrm{CO}_{2}$ fluxes at FLUXNET site level}

The contribution of EM effects to the $\mathrm{CO}_{2}$ fluxes was further explored at the individual FLUXNET site level (Fig. 5.7, Fig. 5.A6, Fig. 5.A7, Fig. 5.A8). Here, we explored the seasonal variations of the EM effects for a set of FLUXNET sites belonging to forest ecosystems in the northern hemisphere, which were found to have the strongest EM effects globally (Fig. 5.3a). This analysis was performed not only for NEE, but also for GPP and ER in order to understand the mechanisms (i.e. photosynthesis or respiratory fluxes) controlling some of the EM effects' features that were observed on the net $\mathrm{CO}_{2}$ fluxes. To ensure the robustness of such an assessment, we only considered sites where the NEE and $\mathrm{NEE}^{\prime}$ estimates (i.e. the difference between ER and GPP) were strongly correlated (i.e. NSE > 0.9) both for the full permutation and no permutation models (Fig. 5.A7b and Fig. 5.A7c). Additionally, we discarded the FLUXNET sites where the correlation coefficient $(\mathrm{r})$ between $\mathrm{NEE}_{\text {(full permutation-no permutation) }}$ and $\mathrm{NEE}^{\prime}$ (full permutation-no permutation) was lower than 0.5 ( $\mathrm{N}=49$ forest sites left after data filtering). We found that both the magnitude, the sign, and the timing of the deviations in $\mathrm{CO}_{2}$ fluxes related to EM effects varied over the course of the seasonal cycle. For instance, considering the temporal context of the seasonal variation resulted in lower net $\mathrm{C}$ uptake at the start and the end of the growing season (i.e. negative difference between full permutation and no permutation). Conversely, one can observe higher net $\mathrm{C}$ uptake (i.e. positive difference between full permutation and no permutation) at the peak of the growing season when capturing past environmental conditions (Fig. 5.7a). The lower net $\mathrm{C}$ uptake at the start of the growing season seemed to be controlled by GPP and to a lesser extent by ER (Fig. 5.7c). On the other hand, the lower net $\mathrm{C}$ uptake at the end of the growing season was driven both by ER and GPP (Fig. 5.7c, d, e, and $\mathrm{f}$ ). It was not entirely clear what were the mechanisms controlling the higher net $\mathrm{C}$ uptake observed at the peak of the growing season. Yet, the absolute seasonal variations (Fig. 5.7b, d, and f) tend to suggest that cumulative EM effects from both GPP and ER may partly explain this observation. The EM effects investigated across $\mathrm{CO}_{2}$ fluxes appeared to be robust as the seasonal variations of the differences between the no permutation and full permutation models for $\mathrm{NEE}^{\prime}$ (Fig. 5.A7) were found to be comparable to the ones found in NEE.

EM effects related to the climate and vegetation of previous years (i.e. comparing permuted year and no permutation) had a small contribution for NEE at the peak of the growing season (Fig. 5.7b), which was less apparent for the gross $\mathrm{CO}_{2}$ fluxes (Fig. 5.7c and f). The low EM effects related to the environmental conditions of previous years suggested that most of the observed EM effects were related to the seasonal variations within a year. However, these findings were contingent on the capacity of the LSTM models to capture anomaly signals and on the magnitude of the anomaly signals presented in the EC data. Furthermore, during the training procedure, the LSTM models have to optimize both spatial and temporal variability, which may limit the capacity of such statistical model to properly capture the $\mathrm{CO}_{2}$ flux variabilities for a specific site. As such, given the number and heterogeneity of FLUXNET sites considered in this study, the observed estimates of EM effects were very likely conservative. 
In this FLUXNET site level analysis ( $\mathrm{N}=104$ sites left after data filtering), we found that the effects of EM observed for ER were overall higher than those observed for GPP (Fig. 5.A8a). It was expected that there would be strong EM effects on the respiratory fluxes as ER is not only sensitive to temperature but also to temporal changes in the $\mathrm{C}$ supply via photosynthesis and the subsequent litterfall that drives litter/soil respiration. This finding suggests that the EM effects on ER are related not only to changes in temperature, which should also impact but to a lesser extent GPP, but also to changes in the C pools. The "flux partitioning" of EM effects is likely to be of substantial use to the modelling community and warrants application to a much larger set of FLUXNET sites to validate the approach used.

\subsection{Conclusions}

This study based on vegetation, climate, and EC flux tower data presents new insights in understanding the contribution of past temporal vegetation and climate dynamics to $\mathrm{CO}_{2}$ fluxes globally. At the FLUXNET site level, we provide insights on the mechanisms controlling the observed EM effects on the seasonal cycle of NEE and find that the EM effects are more apparent in NEE than either on ER or GPP. At the global scale, we find a widespread and substantial contribution of EM effects to NEE across scales, in particular at the seasonal scale. In addition, we show that EM effects prevail in regions with moderate interannual variability of $\mathrm{CO}_{2}$ fluxes, albeit variable responses across the gradient of $\mathrm{CO}_{2}$ flux interannual variability. Global relationships between EM effects and atmospheric and biospheric variables indicate that energy limited regions where there is a large fraction of deciduous and mixed forests experience substantial EM effects on NEE. Conversely, water-limited regions where $\mathrm{CO}_{2}$ fluxes are mainly controlled by instantaneous climatic conditions exhibit relatively low EM effects. By investigating the 2018 European heatwave, we have demonstrated that EM effects dampen the predicted net $\mathrm{CO}_{2}$ flux responses to climate variabilities (i.e. positive or negative anomalies) and that forested areas showed lower EM effects on $\mathrm{CO}_{2}$ fluxes indicating a degree of resilience of these systems to heat waves. Our study highlights the importance of EM effects for the dynamics of the terrestrial $\mathrm{C}$ cycle. As such, it appears important to include past environmental conditions in data-driven statistical approaches that seek to better understand the responses of the terrestrial $\mathrm{C}$ cycle to climate and vegetation changes. 
- full temporal context $\ldots$ inter-annual
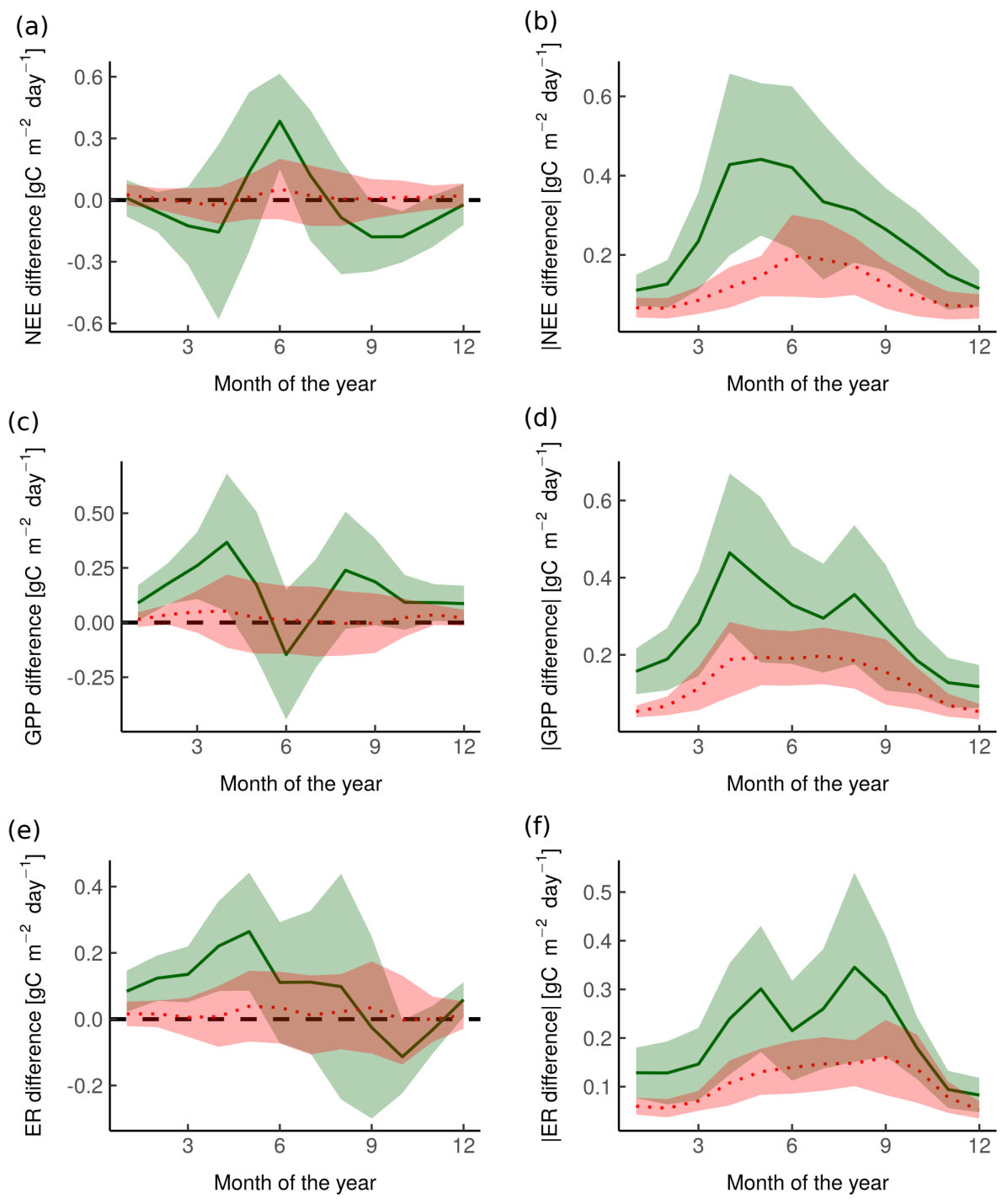

Figure 5.7: Mean seasonal cycle of the actual differences for the full temporal context (i.e. no permutation vs. full permutation models) and interannual variability (i.e. no permutation vs. permuted year models) at deciduous forest FLUXNET sites ( $\mathrm{N}=49$ sites) for NEE, GPP, and ER. Both actual differences (a, c, and e) and absolute differences (b, d, and f) were calculated. Differences were computed for each month independently and from the median estimates of the 15 model runs. Only sites where the NEE and NEE' estimates (i.e. the difference between ER and GPP) were strongly correlated (i.e. NSE $>0.9$ ) for both the full permutation and no permutation models were considered. Additionally, FLUXNET sites where the correlation coefficient (r) between NEE (full permutation - no permutation) and $\mathrm{NEE}^{\prime}$ (full permutation -no permutation) was lower than 0.5 were discarded. Mean \pm standard deviation across all the deciduous forest FLUXNET sites are reported. 


\section{A Appendix}

This appendix represents the supplementary materials of the presented publication.

\section{Sites used in this study}

Table 5.A1: S1 Table. List of sites used in this study. $\mathrm{CSH}=$ closed shrubland, $\mathrm{CRO}=$ cropland, DBF $=$ Deciduous broadleaf forest, $\mathrm{DNF}=$ deciduous needleleaf forest, $\mathrm{GRA}=$ grassland, $\mathrm{EBF}=$ evergreen broadleaf forest, $\mathrm{ENF}=$ evergreen needleleaf forest, $\mathrm{MF}=$ mixed forest, $\mathrm{OSH}=$ open shrubland, $\mathrm{SAV}$ = savanna, WSA = woody savanna, and WET = wetland. The PFT and climate classifications used here are coming from the ancillary data files provided by the La Thuile or the FLUXNET2015 datasets (https://fluxnet.fluxdata.org).

\begin{tabular}{ccccc}
\hline Site ID & Lat $\left[{ }^{\circ} \mathrm{N}\right]$ & Long $\left[{ }^{\circ} \mathrm{E}\right]$ & Climate type & Vegetation type \\
& & & & \\
\hline AR-SLu & -33.465 & -66.460 & Temperate & MF \\
AR-Vir & -28.239 & -56.189 & Temperate & ENF \\
AT-Neu & 47.117 & 11.318 & Boreal & GRA \\
AU-Ade & -13.077 & 131.118 & Tropical & WSA \\
AU-ASM & -22.283 & 133.249 & Arid & ENF \\
AU-Cpr & -34.002 & 140.589 & Arid & SAV \\
AU-Cum & -33.615 & 150.724 & Temperate & EBF \\
AU-DaP & -14.063 & 131.318 & Tropical & GRA \\
AU-DaS & -14.159 & 131.388 & Tropical & SAV \\
AU-Dry & -15.259 & 132.371 & Tropical & SAV \\
AU-Emr & -23.859 & 148.475 & Arid & GRA \\
AU-Fog & -12.545 & 131.307 & Tropical & WET \\
AU-Gin & -31.376 & 115.714 & Temperate & WSA \\
AU-GWW & -30.191 & 120.654 & Arid & SAV \\
AU-How & -12.494 & 131.152 & Tropical & WSA \\
AU-Lox & -34.470 & 140.655 & Arid & DBF \\
AU-RDF & -14.564 & 132.478 & Tropical & WSA \\
AU-Rig & -36.650 & 145.576 & Temperate & GRA \\
AU-Rob & -17.117 & 145.630 & Temperate & EBF \\
AU-Stp & -17.151 & 133.350 & Arid & GRA \\
AU-TTE & -22.287 & 133.640 & Arid & OSH \\
AU-Tum & -35.657 & 148.152 & Temperate & EBF \\
AU-Wac & -37.426 & 145.188 & Temperate & EBF \\
AU-Whr & -36.673 & 145.029 & Temperate & EBF \\
AU-Wom & -37.422 & 144.094 & Temperate & EBF \\
AU-Ync & -34.989 & 146.291 & Arid & GRA \\
BE-Bra & 51.308 & 4.520 & Temperate & MF \\
BE-Jal & 50.564 & 6.073 & Temperate & MF \\
BE-Lon & 50.552 & 4.746 & Temperate & CRO \\
BE-Vie & 50.305 & 5.998 & Temperate & MF \\
BR-Ban & -9.824 & -50.159 & Tropical & EBF \\
\hline & & Continued on next page & \\
& & & &
\end{tabular}


Table 5.A1 - Continued from previous page

\begin{tabular}{|c|c|c|c|c|}
\hline Site ID & Lat $\left[{ }^{\circ} \mathrm{N}\right]$ & Long $\left[{ }^{\circ} \mathrm{E}\right]$ & Climate type & Vegetation type \\
\hline BR-Cax & -1.720 & -51.459 & Tropical & $\mathrm{EBF}$ \\
\hline BR-Ji2 & -10.083 & -61.931 & Tropical & $\mathrm{EBF}$ \\
\hline BR-Ma2 & -2.609 & -60.209 & Tropical & $\mathrm{EBF}$ \\
\hline BR-Sa1 & -2.857 & -54.959 & Tropical & $\mathrm{EBF}$ \\
\hline BR-Sa2 & -3.012 & -54.536 & Tropical & $\mathrm{CRO}$ \\
\hline BR-Sa3 & -3.018 & -54.971 & Tropical & $\mathrm{EBF}$ \\
\hline BR-Sp1 & -21.619 & -47.650 & Tropical & WSA \\
\hline BW-Ma1 & -19.916 & 23.560 & Arid & WSA \\
\hline CA-Ca1 & 49.867 & -125.334 & Temperate & ENF \\
\hline $\mathrm{CA}-\mathrm{Ca} 2$ & 49.870 & -125.291 & Temperate & ENF \\
\hline $\mathrm{CA}-\mathrm{Ca} 3$ & 49.535 & -124.900 & Temperate & ENF \\
\hline CA-Gro & 48.217 & -82.156 & Boreal & $\mathrm{MF}$ \\
\hline CA-Let & 49.709 & -112.940 & Boreal & GRA \\
\hline CA-Man & 55.880 & -98.481 & Boreal & ENF \\
\hline CA-Mer & 45.409 & -75.519 & Boreal & WET \\
\hline CA-NS1 & 55.879 & -98.484 & Boreal & ENF \\
\hline CA-NS2 & 55.906 & -98.525 & Boreal & ENF \\
\hline CA-NS3 & 55.912 & -98.382 & Boreal & ENF \\
\hline CA-NS4 & 55.914 & -98.381 & Boreal & ENF \\
\hline CA-NS5 & 55.863 & -98.485 & Boreal & ENF \\
\hline CA-NS6 & 55.917 & -98.964 & Boreal & $\mathrm{OSH}$ \\
\hline CA-NS7 & 56.636 & -99.948 & Boreal & $\mathrm{OSH}$ \\
\hline CA-Oas & 53.629 & -106.198 & Boreal & DBF \\
\hline CA-Obs & 53.987 & -105.118 & Boreal & ENF \\
\hline CA-Ojp & 53.916 & -104.692 & Boreal & ENF \\
\hline CA-Qcu & 49.267 & -74.037 & Boreal & ENF \\
\hline CA-Qfo & 49.693 & -74.342 & Boreal & ENF \\
\hline CA-SF1 & 54.485 & -105.818 & Boreal & ENF \\
\hline CA-SF2 & 54.254 & -105.877 & Boreal & ENF \\
\hline CA-SF3 & 54.092 & -106.005 & Boreal & OSH \\
\hline CA-SJ1 & 53.908 & -104.656 & Boreal & ENF \\
\hline CA-SJ2 & 53.945 & -104.649 & Boreal & ENF \\
\hline $\mathrm{CA}-\mathrm{SJ} 3$ & 53.876 & -104.645 & Boreal & ENF \\
\hline CA-TP1 & 42.661 & -80.559 & Boreal & ENF \\
\hline CA-TP2 & 42.774 & -80.459 & Boreal & ENF \\
\hline CA-TP3 & 42.707 & -80.348 & Boreal & ENF \\
\hline CA-TP4 & 42.710 & -80.357 & Boreal & ENF \\
\hline CA-TPD & 42.635 & -80.558 & Boreal & DBF \\
\hline CA-WP1 & 54.954 & -112.467 & Boreal & WET \\
\hline CA-WP2 & 55.538 & -112.334 & Boreal & WET \\
\hline CA-WP3 & 54.470 & -113.320 & Boreal & WET \\
\hline CG-Hin & -4.681 & 12.004 & Tropical & $\mathrm{EBF}$ \\
\hline CG-Kis & -4.791 & 11.982 & Tropical & $\mathrm{EBF}$ \\
\hline CG-Tch & -4.289 & 11.656 & Tropical & SAV \\
\hline $\mathrm{CH}-\mathrm{Cha}$ & 47.210 & 8.410 & Temperate & GRA \\
\hline CH-Dav & 46.815 & 9.856 & Tundra & ENF \\
\hline CH-Fru & 47.116 & 8.538 & Temperate & GRA \\
\hline
\end{tabular}


Table 5.A1 - Continued from previous page

\begin{tabular}{|c|c|c|c|c|}
\hline Site ID & Lat $\left[{ }^{\circ} \mathrm{N}\right]$ & Long $\left[{ }^{\circ} \mathrm{E}\right]$ & Climate type & Vegetation type \\
\hline CH-Lae & 47.478 & 8.365 & Temperate & MF \\
\hline $\mathrm{CH}-\mathrm{Oe} 1$ & 47.286 & 7.732 & Temperate & GRA \\
\hline $\mathrm{CH}-\mathrm{Oe} 2$ & 47.286 & 7.734 & Temperate & $\mathrm{CRO}$ \\
\hline CN-Anh & 33.000 & 117.000 & Temperate & DBF \\
\hline CN-Bed & 39.531 & 116.252 & Boreal & $\mathrm{EBF}$ \\
\hline CN-Cha & 42.403 & 128.096 & Boreal & $\mathrm{MF}$ \\
\hline CN-Cng & 44.593 & 123.509 & Boreal & GRA \\
\hline CN-Dan & 30.498 & 91.066 & Tundra & GRA \\
\hline CN-Din & 23.173 & 112.536 & Temperate & $\mathrm{EBF}$ \\
\hline CN-Do1 & 31.517 & 121.961 & Temperate & WET \\
\hline CN-Do2 & 31.585 & 121.903 & Temperate & WET \\
\hline CN-Do3 & 31.517 & 121.972 & Temperate & WET \\
\hline CN-Du1 & 42.046 & 116.671 & Boreal & CRO \\
\hline CN-Du2 & 42.047 & 116.284 & Boreal & GRA \\
\hline CN-Du3 & 42.055 & 116.281 & Boreal & GRA \\
\hline $\mathrm{CN}-\mathrm{Ha} 2$ & 37.609 & 101.327 & Tundra & WET \\
\hline CN-HaM & 37.370 & 101.180 & Tundra & GRA \\
\hline CN-Hny & 29.310 & 112.510 & Temperate & $\mathrm{DBF}$ \\
\hline $\mathrm{CN}-\mathrm{Ku} 1$ & 40.538 & 108.694 & Arid & $\mathrm{EBF}$ \\
\hline $\mathrm{CN}-\mathrm{Ku} 2$ & 40.381 & 108.549 & Arid & $\mathrm{OSH}$ \\
\hline CN-Qia & 26.741 & 115.058 & Temperate & ENF \\
\hline CN-Sw2 & 41.790 & 111.897 & Arid & GRA \\
\hline CN-Xi1 & 43.546 & 116.678 & Boreal & GRA \\
\hline $\mathrm{CN}-\mathrm{Xi} 2$ & 43.554 & 116.671 & Boreal & GRA \\
\hline CZ-BK1 & 49.502 & 18.537 & Boreal & ENF \\
\hline CZ-BK2 & 49.494 & 18.543 & Boreal & GRA \\
\hline CZ-wet & 49.025 & 14.770 & Temperate & WET \\
\hline DE-Akm & 53.866 & 13.683 & Temperate & WET \\
\hline DE-Geb & 51.100 & 10.914 & Temperate & $\mathrm{CRO}$ \\
\hline DE-Gri & 50.950 & 13.513 & Temperate & GRA \\
\hline DE-Hai & 51.079 & 10.453 & Temperate & DBF \\
\hline DE-Har & 47.934 & 7.601 & Temperate & ENF \\
\hline DE-Kli & 50.893 & 13.522 & Temperate & $\mathrm{CRO}$ \\
\hline DE-Lkb & 49.100 & 13.305 & Temperate & ENF \\
\hline DE-Lnf & 51.328 & 10.368 & Temperate & DBF \\
\hline DE-Meh & 51.275 & 10.655 & Temperate & $\mathrm{MF}$ \\
\hline DE-Obe & 50.787 & 13.721 & Temperate & ENF \\
\hline DE-RuR & 50.622 & 6.304 & Temperate & GRA \\
\hline DE-RuS & 50.866 & 6.447 & Temperate & CRO \\
\hline DE-Seh & 50.871 & 6.450 & Temperate & CRO \\
\hline DE-SfN & 47.806 & 11.328 & Temperate & WET \\
\hline DE-Spw & 51.892 & 14.034 & Temperate & WET \\
\hline DE-Tha & 50.962 & 13.565 & Temperate & ENF \\
\hline DE-Wet & 50.453 & 11.458 & Temperate & ENF \\
\hline DE-Zrk & 53.876 & 12.889 & Temperate & WET \\
\hline DK-Eng & 55.691 & 12.192 & Temperate & GRA \\
\hline DK-Fou & 56.484 & 9.587 & Temperate & $\mathrm{CRO}$ \\
\hline
\end{tabular}


Table 5.A1 - Continued from previous page

\begin{tabular}{|c|c|c|c|c|}
\hline Site ID & Lat $\left[{ }^{\circ} \mathrm{N}\right]$ & Long $\left[{ }^{\circ} \mathrm{E}\right]$ & Climate type & Vegetation type \\
\hline DK-Lva & 55.683 & 12.083 & Temperate & GRA \\
\hline DK-NuF & 64.131 & -51.386 & Tundra & WET \\
\hline DK-Ris & 55.530 & 12.097 & Temperate & $\mathrm{CRO}$ \\
\hline DK-Sor & 55.486 & 11.645 & Temperate & DBF \\
\hline DK-ZaF & 74.481 & -20.555 & Tundra & WET \\
\hline DK-ZaH & 74.473 & -20.550 & Tundra & GRA \\
\hline ES-Amo & 36.834 & -2.252 & Arid & $\mathrm{OSH}$ \\
\hline ES-ES1 & 39.346 & -0.319 & Temperate & ENF \\
\hline ES-ES2 & 39.276 & -0.315 & Temperate & $\mathrm{CRO}$ \\
\hline ES-LgS & 37.098 & -2.966 & Temperate & $\mathrm{OSH}$ \\
\hline ES-LJu & 36.927 & -2.752 & Temperate & $\mathrm{OSH}$ \\
\hline ES-LMa & 39.941 & -5.773 & Temperate & SAV \\
\hline ES-Ln2 & 36.970 & -3.476 & Temperate & $\mathrm{OSH}$ \\
\hline ES-VDA & 42.152 & 1.448 & Temperate & GRA \\
\hline FI-Hyy & 61.847 & 24.295 & Boreal & ENF \\
\hline FI-Jok & 60.899 & 23.513 & Boreal & $\mathrm{CRO}$ \\
\hline FI-Kaa & 69.141 & 27.270 & Boreal & WET \\
\hline FI-Let & 60.642 & 23.960 & Boreal & ENF \\
\hline FI-Lom & 67.997 & 24.209 & Boreal & WET \\
\hline FI-Sii & 61.833 & 24.193 & Boreal & WET \\
\hline FI-Sod & 67.362 & 26.639 & Boreal & ENF \\
\hline FR-Aur & 43.550 & 1.106 & Temperate & $\mathrm{CRO}$ \\
\hline FR-Fon & 48.476 & 2.780 & Temperate & $\mathrm{DBF}$ \\
\hline FR-Gri & 48.844 & 1.952 & Temperate & CRO \\
\hline FR-Hes & 48.674 & 7.065 & Temperate & DBF \\
\hline FR-Lam & 43.496 & 1.238 & Temperate & $\mathrm{CRO}$ \\
\hline FR-LBr & 44.717 & -0.769 & Temperate & ENF \\
\hline FR-Lq1 & 45.644 & 2.735 & Temperate & GRA \\
\hline FR-Lq2 & 45.639 & 2.737 & Temperate & GRA \\
\hline FR-Pue & 43.741 & 3.596 & Temperate & $\mathrm{EBF}$ \\
\hline GF-Guy & 5.279 & -52.925 & Tropical & $\mathrm{EBF}$ \\
\hline GH-Ank & 5.269 & -2.694 & Tropical & $\mathrm{EBF}$ \\
\hline HU-Bug & 46.691 & 19.601 & Temperate & GRA \\
\hline HU-Mat & 47.847 & 19.726 & Temperate & GRA \\
\hline ID-Pag & 2.345 & 114.036 & Tropical & $\mathrm{EBF}$ \\
\hline IE-Ca1 & 52.859 & -6.918 & Temperate & $\mathrm{CRO}$ \\
\hline IE-Dri & 51.987 & -8.752 & Temperate & GRA \\
\hline IL-Yat & 31.345 & 35.052 & Arid & ENF \\
\hline IT-Amp & 41.904 & 13.605 & Temperate & GRA \\
\hline IT-BCi & 40.524 & 14.957 & Temperate & CRO \\
\hline IT-Be2 & 46.004 & 13.028 & Temperate & $\mathrm{CRO}$ \\
\hline IT-Bon & 39.478 & 16.535 & Temperate & ENF \\
\hline IT-CA1 & 42.380 & 12.027 & Temperate & DBF \\
\hline IT-CA2 & 42.377 & 12.026 & Temperate & CRO \\
\hline IT-CA3 & 42.380 & 12.022 & Temperate & $\mathrm{DBF}$ \\
\hline IT-Cas & 45.070 & 8.718 & Temperate & CRO \\
\hline IT-Col & 41.849 & 13.588 & Temperate & DBF \\
\hline
\end{tabular}


Table 5.A1 - Continued from previous page

\begin{tabular}{|c|c|c|c|c|}
\hline Site ID & Lat $\left[{ }^{\circ} \mathrm{N}\right]$ & Long $\left[{ }^{\circ} \mathrm{E}\right]$ & Climate type & Vegetation type \\
\hline IT-Cp2 & 41.704 & 12.357 & Temperate & EBF \\
\hline IT-Cpz & 41.705 & 12.376 & Temperate & $\mathrm{EBF}$ \\
\hline IT-Isp & 45.813 & 8.634 & Temperate & DBF \\
\hline IT-La2 & 45.954 & 11.285 & Temperate & ENF \\
\hline IT-Lav & 45.956 & 11.281 & Temperate & ENF \\
\hline IT-Lec & 43.304 & 11.270 & Temperate & $\mathrm{EBF}$ \\
\hline IT-LMa & 45.153 & 7.583 & Temperate & $\mathrm{DBF}$ \\
\hline IT-Mal & 46.114 & 11.703 & Temperate & GRA \\
\hline IT-MBo & 46.015 & 11.046 & Boreal & GRA \\
\hline IT-Noe & 40.606 & 8.151 & Temperate & $\mathrm{CSH}$ \\
\hline IT-Non & 44.690 & 11.091 & Temperate & $\mathrm{DBF}$ \\
\hline IT-Pia & 42.584 & 10.078 & Temperate & $\mathrm{OSH}$ \\
\hline IT-PT1 & 45.201 & 9.061 & Temperate & $\mathrm{DBF}$ \\
\hline IT-Ren & 46.587 & 11.434 & Boreal & ENF \\
\hline IT-Ro1 & 42.408 & 11.930 & Temperate & $\mathrm{DBF}$ \\
\hline IT-Ro2 & 42.390 & 11.921 & Temperate & $\mathrm{DBF}$ \\
\hline IT-SR2 & 43.732 & 10.291 & Temperate & ENF \\
\hline IT-SRo & 43.728 & 10.284 & Temperate & ENF \\
\hline IT-Tor & 45.844 & 7.578 & Boreal & GRA \\
\hline IT-Vig & 45.296 & 8.876 & Temperate & $\mathrm{DBF}$ \\
\hline JP-Mas & 36.054 & 140.027 & Temperate & CRO \\
\hline JP-MBF & 44.384 & 142.319 & Boreal & $\mathrm{DBF}$ \\
\hline JP-SMF & 35.262 & 137.079 & Temperate & $\mathrm{MF}$ \\
\hline JP-Tak & 36.146 & 137.423 & Boreal & $\mathrm{DBF}$ \\
\hline JP-Tef & 45.056 & 142.106 & Boreal & $\mathrm{MF}$ \\
\hline JP-Tom & 42.739 & 141.515 & Boreal & $\mathrm{MF}$ \\
\hline KR-Hnm & 34.550 & 126.570 & Temperate & CRO \\
\hline KR-Kw1 & 37.749 & 127.163 & Boreal & $\mathrm{MF}$ \\
\hline ML-AgG & 15.343 & -1.481 & Arid & GRA \\
\hline ML-Kem & 15.224 & -1.566 & Arid & DBF \\
\hline MY-PSO & 2.973 & 102.306 & Tropical & $\mathrm{EBF}$ \\
\hline NL-Ca1 & 51.971 & 4.927 & Temperate & GRA \\
\hline NL-Haa & 52.004 & 4.806 & Temperate & GRA \\
\hline NL-Hor & 52.240 & 5.071 & Temperate & GRA \\
\hline NL-Lan & 51.954 & 4.903 & Temperate & CRO \\
\hline NL-Loo & 52.167 & 5.744 & Temperate & ENF \\
\hline NL-Lut & 53.399 & 6.356 & Temperate & CRO \\
\hline NL-Mol & 51.650 & 4.639 & Temperate & $\mathrm{CRO}$ \\
\hline NO-Adv & 78.186 & 15.923 & Tundra & WET \\
\hline PA-SPn & 9.318 & -79.635 & Tropical & $\mathrm{DBF}$ \\
\hline PA-SPs & 9.314 & -79.631 & Tropical & GRA \\
\hline PL-Wet & 52.762 & 16.309 & Temperate & WET \\
\hline PT-Esp & 38.639 & -8.602 & Temperate & $\mathrm{EBF}$ \\
\hline PT-Mi1 & 38.541 & -8.000 & Temperate & $\mathrm{EBF}$ \\
\hline PT-Mi2 & 38.477 & -8.025 & Temperate & GRA \\
\hline RU-Che & 68.613 & 161.341 & Boreal & WET \\
\hline RU-Cok & 70.829 & 147.494 & Boreal & $\mathrm{OSH}$ \\
\hline
\end{tabular}


Table 5.A1 - Continued from previous page

\begin{tabular}{|c|c|c|c|c|}
\hline Site ID & Lat $\left[{ }^{\circ} \mathrm{N}\right]$ & Long $\left[{ }^{\circ} \mathrm{E}\right]$ & Climate type & Vegetation type \\
\hline RU-Fyo & 56.462 & 32.922 & Boreal & ENF \\
\hline RU-Ha1 & 54.725 & 90.002 & Boreal & GRA \\
\hline RU-Ha2 & 54.773 & 89.957 & Boreal & GRA \\
\hline RU-Ha3 & 54.705 & 89.078 & Boreal & GRA \\
\hline RU-Sam & 72.374 & 126.496 & Boreal & GRA \\
\hline RU-Tks & 71.594 & 128.888 & Boreal & GRA \\
\hline RU-Vrk & 67.055 & 62.940 & Boreal & $\mathrm{CSH}$ \\
\hline RU-Zot & 60.801 & 89.351 & Boreal & ENF \\
\hline SD-Dem & 13.283 & 30.478 & Arid & SAV \\
\hline SE-Abi & 68.362 & 18.795 & Tundra & DBF \\
\hline SE-Deg & 64.182 & 19.557 & Boreal & WET \\
\hline SE-Faj & 56.265 & 13.554 & Temperate & WET \\
\hline SE-Fla & 64.113 & 19.457 & Boreal & ENF \\
\hline SE-Nor & 60.086 & 17.480 & Boreal & ENF \\
\hline SE-Sk1 & 60.125 & 17.918 & Boreal & ENF \\
\hline SE-Sk2 & 60.130 & 17.840 & Boreal & ENF \\
\hline SE-St1 & 68.354 & 19.050 & Tundra & WET \\
\hline SK-Tat & 49.121 & 20.163 & Boreal & ENF \\
\hline SN-Dhr & 15.403 & -15.432 & Arid & SAV \\
\hline TW-Tar & 24.031 & 120.688 & Temperate & CRO \\
\hline UK-AMo & 55.792 & -3.244 & Temperate & WET \\
\hline UK-EBu & 55.866 & -3.206 & Temperate & GRA \\
\hline UK-ESa & 55.907 & -2.859 & Temperate & $\mathrm{CRO}$ \\
\hline UK-Gri & 56.607 & -3.798 & Temperate & ENF \\
\hline UK-Ham & 51.154 & -0.858 & Temperate & DBF \\
\hline UK-Her & 51.784 & -0.476 & Temperate & $\mathrm{CRO}$ \\
\hline UK-PL3 & 51.450 & -1.267 & Temperate & DBF \\
\hline UK-Tad & 51.207 & -2.829 & Temperate & GRA \\
\hline US-AR1 & 36.427 & -99.420 & Temperate & GRA \\
\hline US-AR2 & 36.636 & -99.597 & Temperate & GRA \\
\hline US-ARb & 35.550 & -98.040 & Temperate & GRA \\
\hline US-ARc & 35.547 & -98.040 & Temperate & GRA \\
\hline US-ARM & 36.606 & -97.489 & Temperate & CRO \\
\hline US-Atq & 70.470 & -157.409 & Tundra & WET \\
\hline US-Aud & 31.591 & -110.510 & Arid & GRA \\
\hline US-Bar & 44.065 & -71.288 & Boreal & DBF \\
\hline US-Bkg & 44.345 & -96.836 & Boreal & GRA \\
\hline US-Blo & 38.895 & -120.633 & Temperate & ENF \\
\hline US-Bn1 & 63.920 & -145.378 & Boreal & ENF \\
\hline US-Bn2 & 63.920 & -145.378 & Boreal & DBF \\
\hline US-Bn3 & 63.923 & -145.744 & Boreal & $\mathrm{OSH}$ \\
\hline US-Bo1 & 40.006 & -88.290 & Boreal & CRO \\
\hline US-Bo2 & 40.009 & -88.290 & Boreal & $\mathrm{CRO}$ \\
\hline US-Brw & 71.323 & -156.609 & Tundra & WET \\
\hline US-CaV & 39.063 & -79.421 & Temperate & GRA \\
\hline US-Cop & 38.090 & -109.390 & Boreal & GRA \\
\hline US-CRT & 41.629 & -83.347 & Boreal & CRO \\
\hline
\end{tabular}


Table 5.A1 - Continued from previous page

\begin{tabular}{|c|c|c|c|c|}
\hline Site ID & Lat $\left[{ }^{\circ} \mathrm{N}\right]$ & Long $\left[{ }^{\circ} \mathrm{E}\right]$ & Climate type & Vegetation type \\
\hline US-Dk1 & 35.971 & -79.093 & Temperate & GRA \\
\hline US-Dk2 & 35.974 & -79.100 & Temperate & $\mathrm{DBF}$ \\
\hline US-Dk3 & 35.978 & -79.094 & Temperate & ENF \\
\hline US-Fmf & 35.143 & -111.727 & Temperate & ENF \\
\hline US-FPe & 48.308 & -105.102 & Arid & GRA \\
\hline US-FR2 & 29.950 & -97.996 & Temperate & WSA \\
\hline US-Fuf & 35.089 & -111.762 & Temperate & ENF \\
\hline US-Fwf & 35.445 & -111.772 & Temperate & GRA \\
\hline US-GBT & 41.366 & -106.240 & Boreal & ENF \\
\hline US-GLE & 41.367 & -106.240 & Boreal & ENF \\
\hline US-Goo & 34.255 & -89.874 & Temperate & GRA \\
\hline US-Ha1 & 42.538 & -72.171 & Boreal & $\mathrm{DBF}$ \\
\hline US-Ha2 & 42.539 & -72.178 & Boreal & ENF \\
\hline US-Ho1 & 45.204 & -68.740 & Boreal & ENF \\
\hline US-Ho2 & 45.209 & -68.747 & Boreal & ENF \\
\hline US-IB1 & 41.859 & -88.223 & Boreal & $\mathrm{CRO}$ \\
\hline US-IB2 & 41.841 & -88.241 & Boreal & GRA \\
\hline US-Ivo & 68.487 & -155.750 & Tundra & WET \\
\hline US-KS1 & 28.458 & -80.671 & Temperate & ENF \\
\hline US-KS2 & 28.609 & -80.671 & Temperate & $\mathrm{CSH}$ \\
\hline US-Lin & 36.357 & -119.842 & Arid & $\mathrm{CRO}$ \\
\hline US-Los & 46.083 & -89.979 & Boreal & WET \\
\hline US-LPH & 42.542 & -72.185 & Boreal & $\mathrm{DBF}$ \\
\hline US-Me1 & 44.579 & -121.500 & Temperate & ENF \\
\hline US-Me2 & 44.452 & -121.557 & Temperate & ENF \\
\hline US-Me3 & 44.315 & -121.608 & Temperate & ENF \\
\hline US-Me4 & 44.499 & -121.622 & Temperate & ENF \\
\hline US-Me5 & 44.437 & -121.567 & Temperate & ENF \\
\hline US-Me6 & 44.323 & -121.608 & Temperate & ENF \\
\hline US-MMS & 39.323 & -86.413 & Temperate & $\mathrm{DBF}$ \\
\hline US-MOz & 38.744 & -92.200 & Temperate & $\mathrm{DBF}$ \\
\hline US-Myb & 38.050 & -121.765 & Temperate & WET \\
\hline US-NC1 & 35.812 & -76.712 & Temperate & $\mathrm{OSH}$ \\
\hline US-NC2 & 35.803 & -76.668 & Temperate & ENF \\
\hline US-Ne1 & 41.165 & -96.477 & Boreal & CRO \\
\hline US-Ne2 & 41.165 & -96.470 & Boreal & $\mathrm{CRO}$ \\
\hline US-Ne3 & 41.180 & -96.440 & Boreal & $\mathrm{CRO}$ \\
\hline US-NR1 & 40.033 & -105.546 & Boreal & ENF \\
\hline US-Oho & 41.554 & -83.844 & Boreal & $\mathrm{DBF}$ \\
\hline US-ORv & 40.020 & -83.018 & Boreal & WET \\
\hline US-PFa & 45.946 & -90.272 & Boreal & $\mathrm{MF}$ \\
\hline US-Prr & 65.124 & -147.488 & Boreal & ENF \\
\hline US-SO2 & 33.374 & -116.623 & Temperate & $\mathrm{CSH}$ \\
\hline US-SO3 & 33.377 & -116.623 & Temperate & $\mathrm{CSH}$ \\
\hline US-SO4 & 33.385 & -116.641 & Temperate & $\mathrm{CSH}$ \\
\hline US-SP1 & 29.738 & -82.219 & Temperate & ENF \\
\hline US-SP2 & 29.765 & -82.245 & Temperate & ENF \\
\hline
\end{tabular}


Table 5.A1 - Continued from previous page

\begin{tabular}{|c|c|c|c|c|}
\hline Site ID & Lat $\left[{ }^{\circ} \mathrm{N}\right]$ & Long $\left[{ }^{\circ} \mathrm{E}\right]$ & Climate type & Vegetation type \\
\hline US-SP3 & 29.755 & -82.163 & Temperate & ENF \\
\hline US-SRC & 31.908 & -110.840 & Arid & $\mathrm{OSH}$ \\
\hline US-SRG & 31.789 & -110.828 & Arid & GRA \\
\hline US-SRM & 31.821 & -110.866 & Arid & WSA \\
\hline US-Sta & 41.397 & -106.802 & Boreal & $\mathrm{OSH}$ \\
\hline US-Syv & 46.242 & -89.348 & Boreal & MF \\
\hline US-Ton & 38.432 & -120.966 & Temperate & WSA \\
\hline US-Tw1 & 38.107 & -121.647 & Temperate & WET \\
\hline US-Tw2 & 38.105 & -121.643 & Temperate & $\mathrm{CRO}$ \\
\hline US-Tw3 & 38.116 & -121.647 & Temperate & $\mathrm{CRO}$ \\
\hline US-Tw4 & 38.103 & -121.641 & Temperate & WET \\
\hline US-Twt & 38.109 & -121.653 & Temperate & CRO \\
\hline US-UMB & 45.560 & -84.714 & Boreal & DBF \\
\hline US-UMd & 45.562 & -84.698 & Boreal & $\mathrm{DBF}$ \\
\hline US-Var & 38.413 & -120.951 & Temperate & GRA \\
\hline US-WCr & 45.806 & -90.080 & Boreal & DBF \\
\hline US-Whs & 31.744 & -110.052 & Arid & $\mathrm{OSH}$ \\
\hline US-Wi0 & 46.619 & -91.081 & Boreal & ENF \\
\hline US-Wi1 & 46.730 & -91.233 & Boreal & DBF \\
\hline US-Wi3 & 46.635 & -91.099 & Boreal & DBF \\
\hline US-Wi4 & 46.739 & -91.166 & Boreal & ENF \\
\hline US-Wi5 & 46.653 & -91.086 & Boreal & ENF \\
\hline US-Wi6 & 46.625 & -91.298 & Boreal & $\mathrm{OSH}$ \\
\hline US-Wi7 & 46.649 & -91.069 & Boreal & $\mathrm{OSH}$ \\
\hline US-Wi8 & 46.722 & -91.252 & Boreal & DBF \\
\hline US-Wi9 & 46.619 & -91.081 & Boreal & ENF \\
\hline US-Wkg & 31.736 & -109.942 & Arid & GRA \\
\hline US-WPT & 41.465 & -82.996 & Boreal & WET \\
\hline US-Wrc & 45.821 & -121.952 & Temperate & ENF \\
\hline VU-Coc & -15.443 & 167.192 & Tropical & $\mathrm{EBF}$ \\
\hline ZA-Kru & -25.020 & 31.497 & Temperate & SAV \\
\hline ZA-Map & -23.833 & 31.214 & Temperate & SAV \\
\hline ZM-Mon & -15.438 & 23.253 & Tropical & DBF \\
\hline
\end{tabular}


Performance of the gap-filling procedure for the differtent vegetation indices

(a)

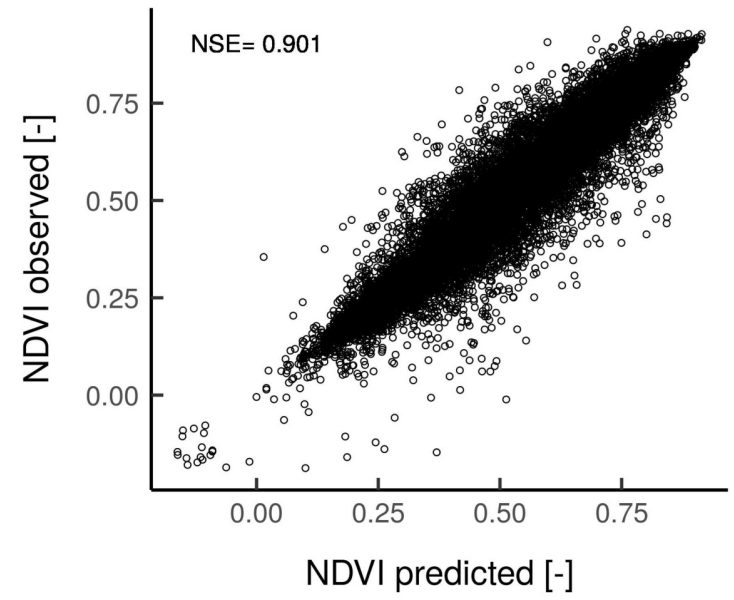

(b)

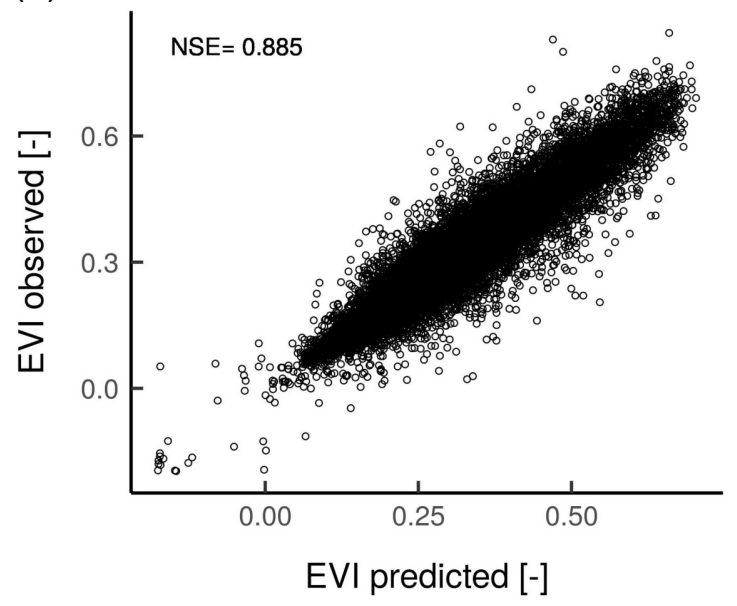

Figure 5.A1: Performance of the gap-filling procedure of NDVI and EVI using a RF model and climate variables (i.e. $\mathbf{T}_{\text {air }}$, Rg, and rpot) as predictive variables. The model was trained on $70 \%$ of the data and evaluated on $30 \%$ of the left out data. NDVI = normalized difference vegetation index and $\mathrm{EVI}=$ enhanced vegetation index. 
Performance of the gap-filling procedure for the differtent climate variables.
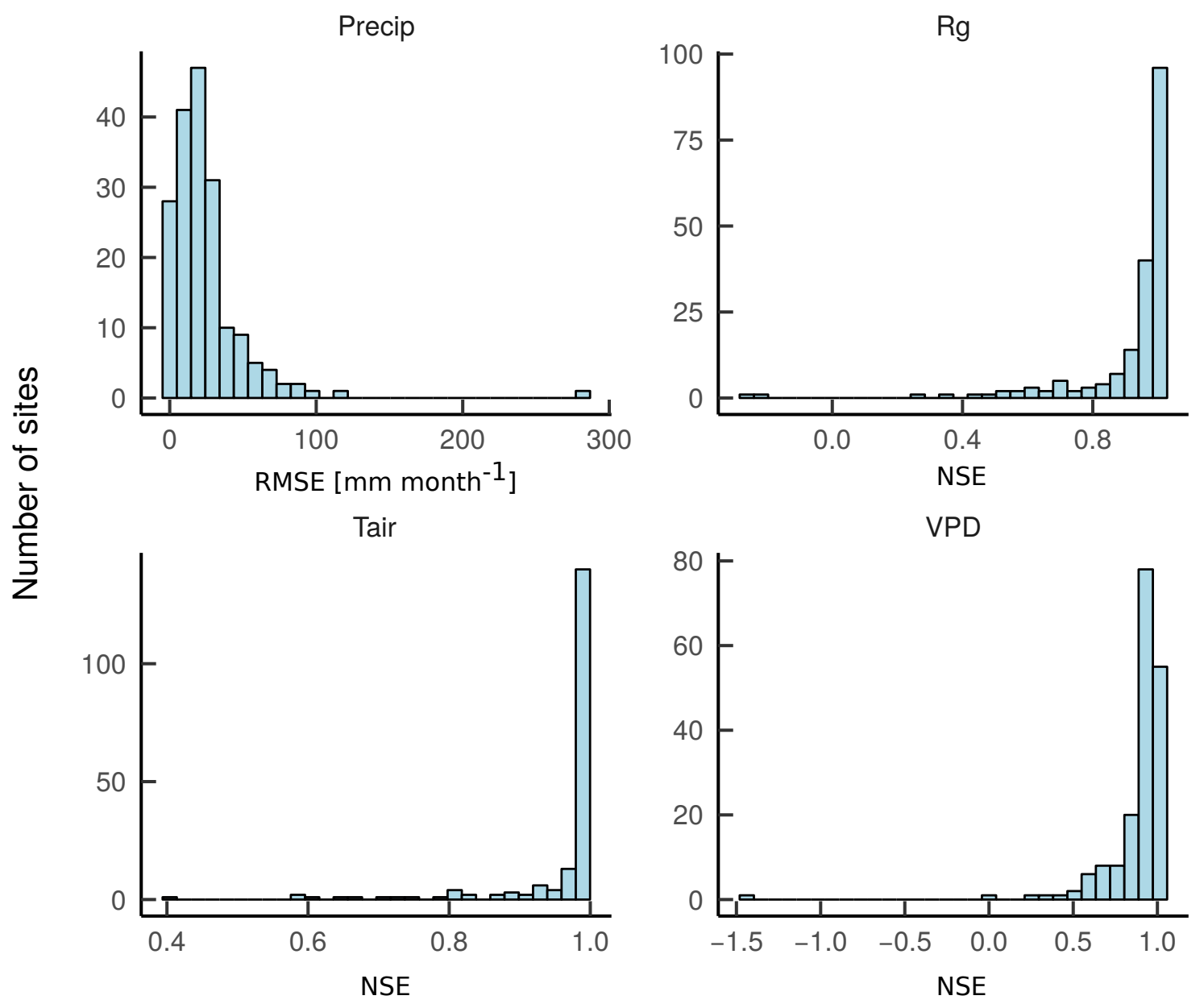

Figure 5.A2: Performance of the gap-filling procedure for the different climate variables. Assessment of the gap-filling procedure was done for $\mathrm{T}_{\text {air }}$, Precip, Rg, and VPD. For $\mathrm{T}_{\text {air }}$, Rg, and VPD, the Nash-Sutcliffe efficiency (NSE) is reported, while the root mean square error (RMSE) is reported for Precip. 
Global spatial patterns and time series for 2001-2018 of the no permutation and the full permutation models
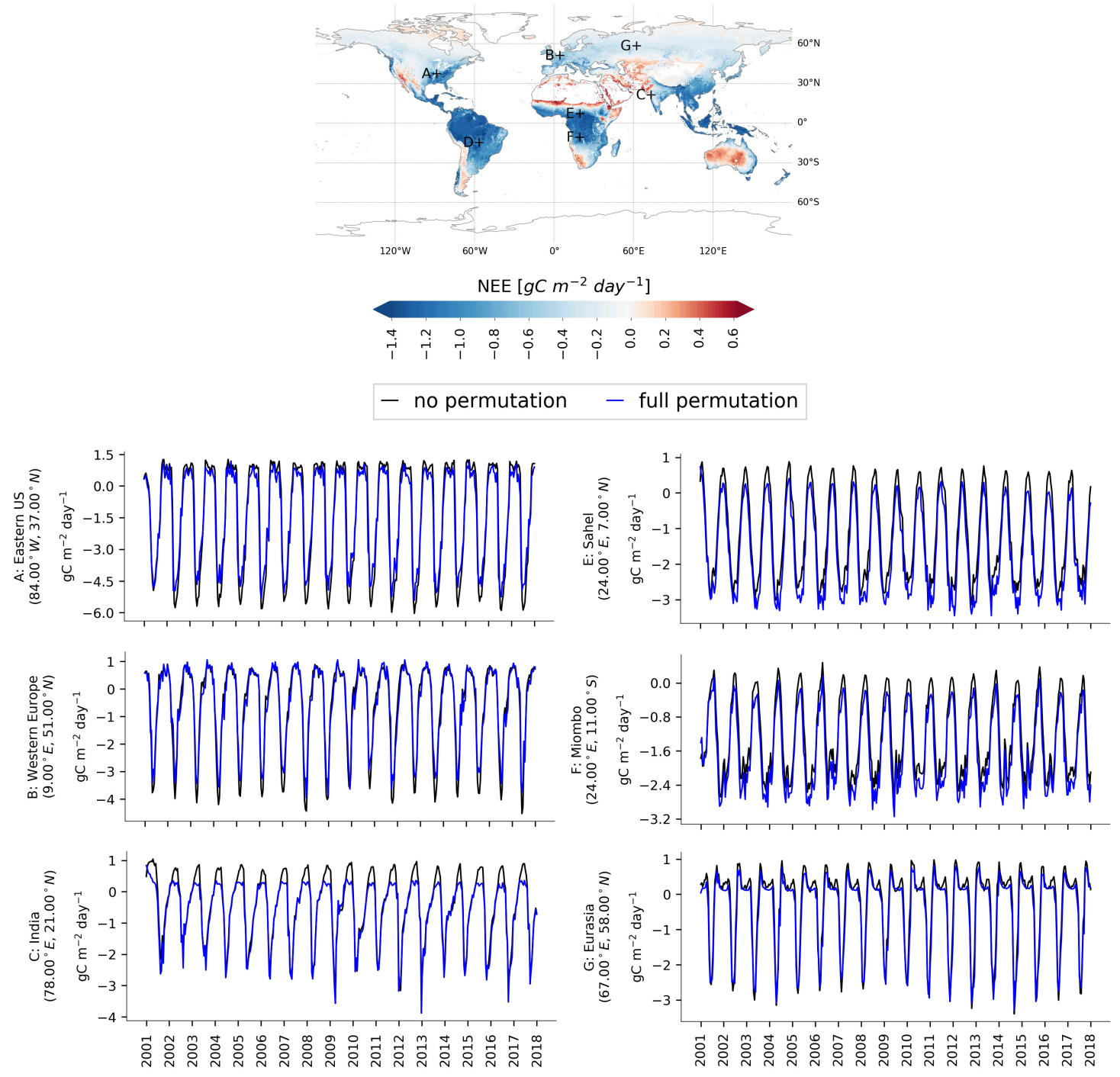

Figure 5.A3: Global spatial patterns of NEE and the temporal variations in selected locations derived from the no permutation and the full permutation models. Global spatial maps were derived from the no permutation product. Line plots depict time series of the no permutation (black) and the full permutation (blue) models for selected locations (median values within a $0.5^{\circ}$ bounding box around selected locations) from 2001 to 2018 in $\mathrm{gC} \mathrm{m}^{-2}$ day $^{-1}$. 
Mean seasonal cycle of the no permutation and the full permutation models

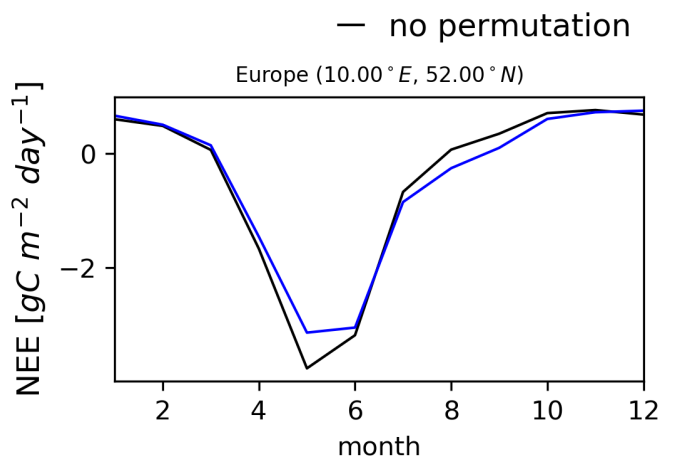

- full permutation

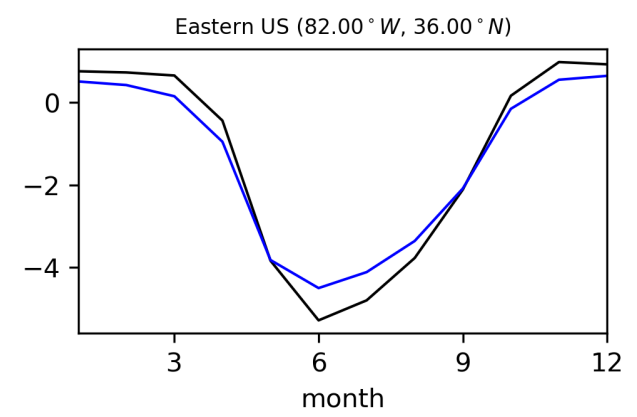

Figure 5.A4: Mean seasonal cycle of the no permutation and the full permutation models for two selected locations. Line plots depict the mean seasonal cycle of the no permutation (black) and the full permutation (blue) models for selected locations (median values within a $0.5^{\circ}$ bounding box around selected locations). 


\section{European heatwave}

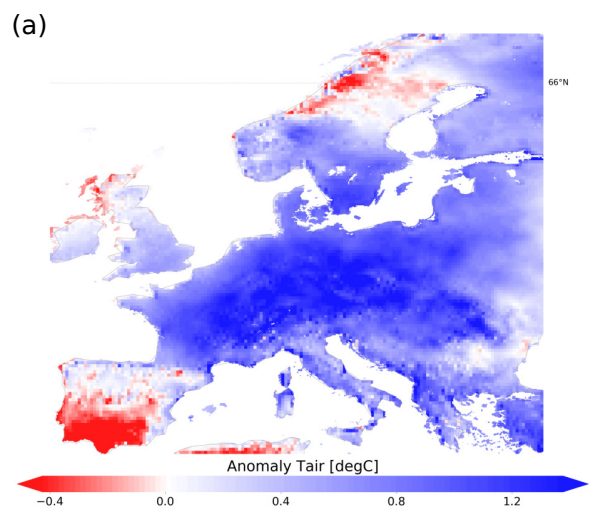

(c)

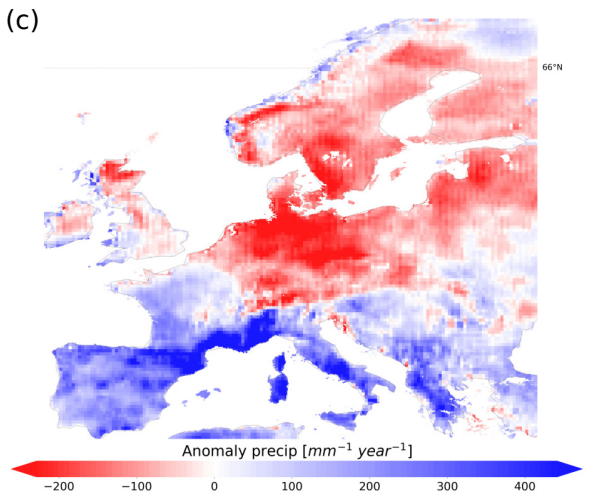

(e) (b)

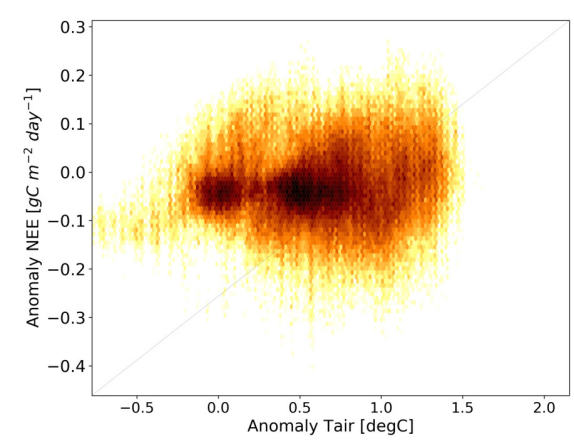

(d)

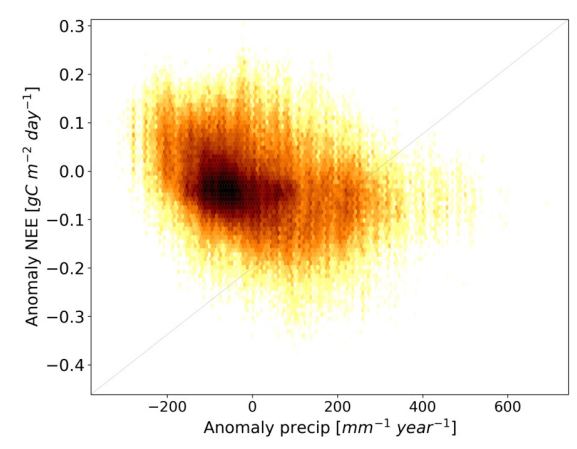

(f)
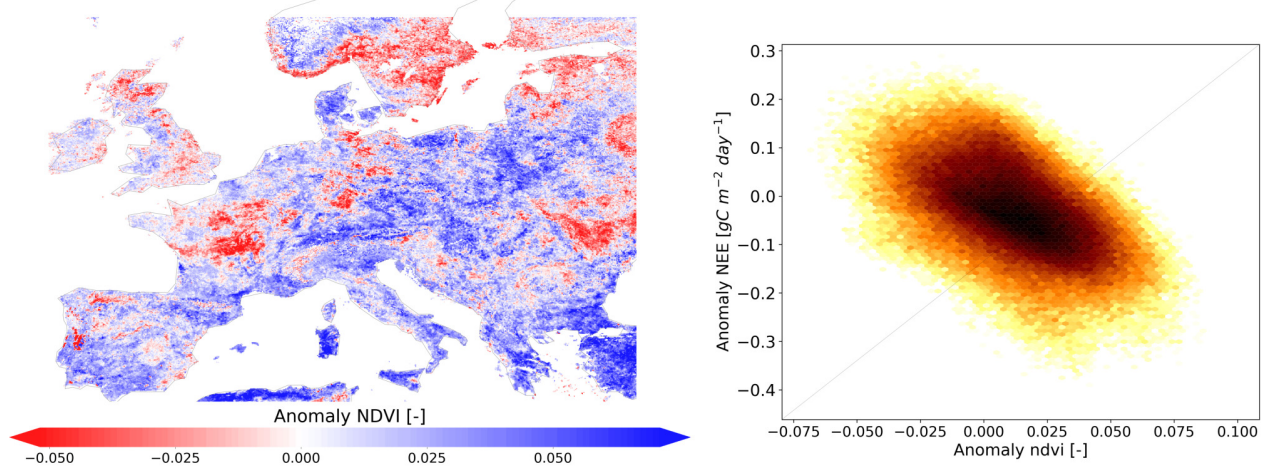

Figure 5.A5: Anomalies in $T_{a i r}$ (a), Precip (c), and NDVI (e) for the year 2018. Comparisons of the anomalies in NEE from the no permutation model against $\mathrm{T}_{\text {air }}$ (b), Precip (d), and NDVI (f) are also shown. Anomalies were computed by calculating the difference between annual estimates in 2018 and mean annual estimates over the period 2001-2017 (except 2003) 


\section{Cross-validation results of the LSTM models}

(a)

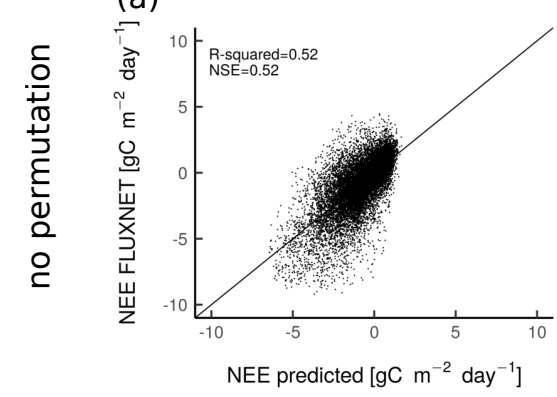

(d)

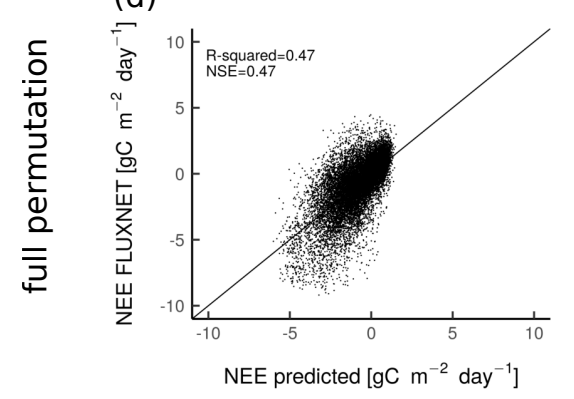

(b)

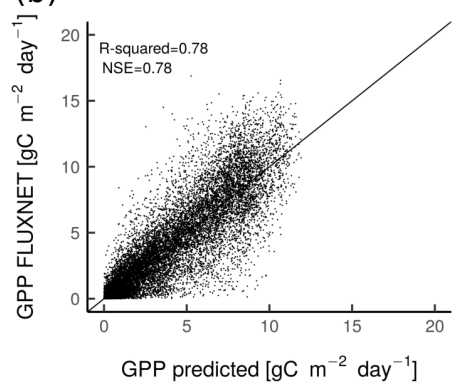

(e)

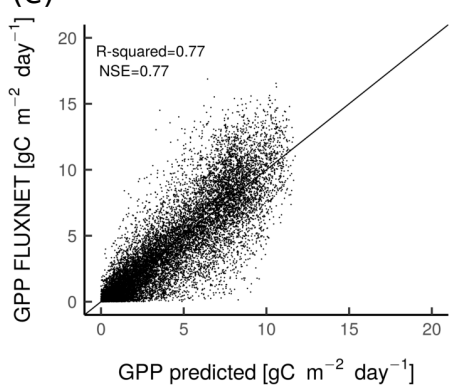

(c)

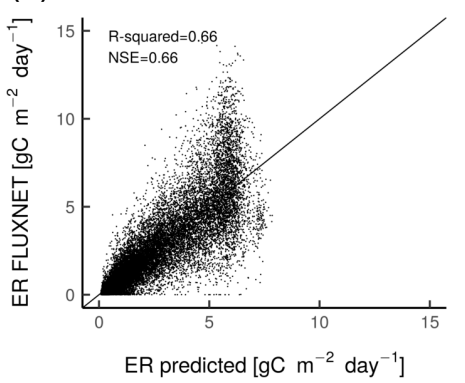

(f)

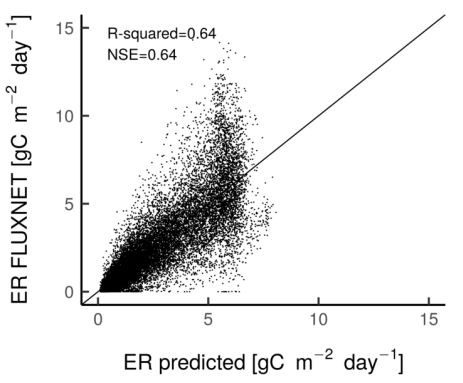

Figure 5.A6: Scatterplots of observed data and the LSTM modeled fluxes for NEE (a, d), GPP $(\mathbf{b}, \mathbf{e})$, and ER (c, f). The modeled estimates were derived from the median of the ensemble of the 15 model simulations. 


\section{Difference between no permutation and full permutation for $\mathrm{NEE}^{\prime}$}
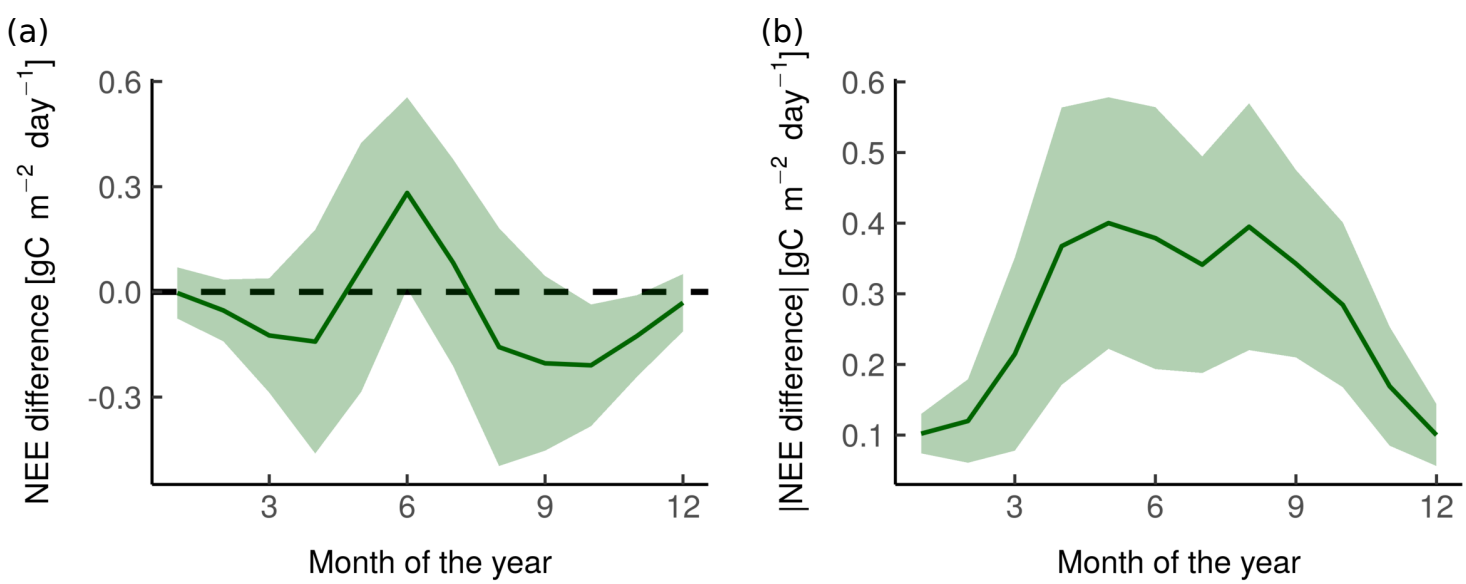

Figure 5.A7: Mean seasonal cycle of the actual differences for the full temporal context (i.e. no permutation vs. full permutation models) at deciduous forest FLUXNET sites ( $\mathrm{N}=49$ sites) for NEE' (ER - GPP). Both actual differences (a) and absolute differences (b) were calculated. Differences were computed for each month independently and from the median estimates of the 15 model simulations. Only sites where the NEE and NEE' estimates (i.e. the difference between predicted ER and GPP) were strongly correlated (i.e. NSE $>0.9$ ) for both the full permutation and no permutation models were considered. Additionally, FLUXNET sites where the correlation coefficient (r) between NEE (full permutation - no permutation) and $\mathrm{NEE}^{\prime}$ (full permutation -no permutation) was lower than 0.5 were discarded. Mean \pm standard deviation across all the FLUXNET sites are reported. 


\section{Comparison between net ecosystem exchange (NEE) and NEE'}
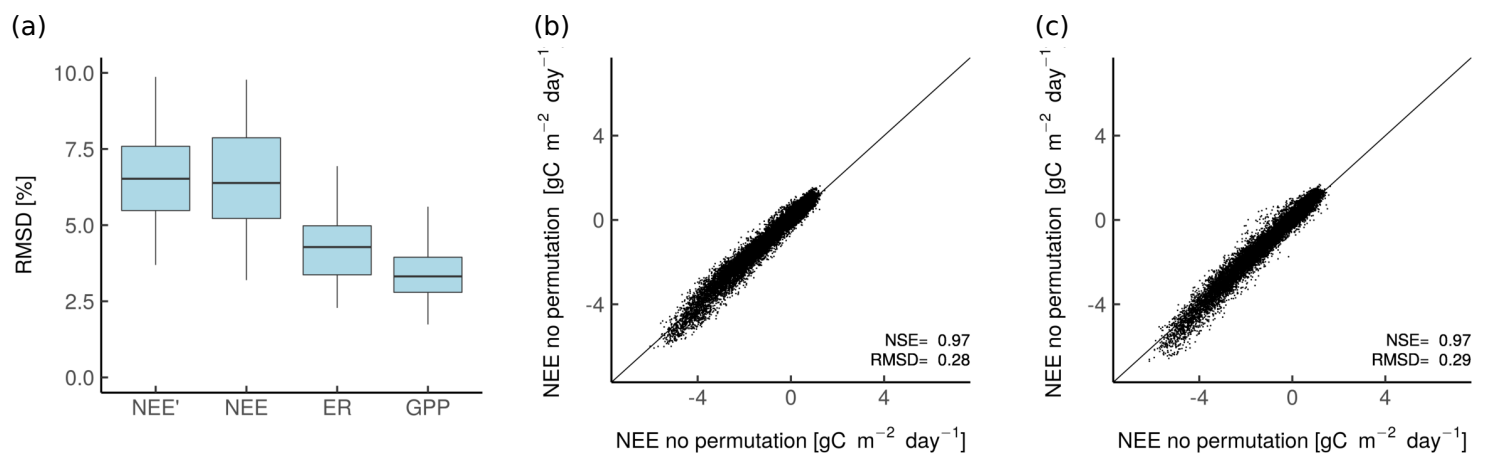

Figure 5.A8: Boxplots of RMSD across $\mathrm{CO}_{2}$ fluxes (a). Scatterplots of NEE and $\mathrm{NEE}^{\prime}$ (ER GPP) at Fluxnet site level ( $\mathrm{N}=104$ sites) for the no permutation (b) and full permutation models (c). To ensure a fair comparison of the RMSD estimates across fluxes (a), each of the $\mathrm{CO}_{2}$ flux estimates were first normalized independently for each FLUXNET site from 0 to 1 before computing the RMSD estimates. Only sites where the NEE and NEE' estimates (i.e. the difference between ER and GPP) were strongly correlated (i.e. NSE $>0.9$ ) for both the full permutation and no permutation models were considered. Additionally, FLUXNET sites where the correlation coefficient (r) between NEE (full permutation - no permutation) and $\mathrm{NEE}^{\prime}$ (full permutation -no permutation) was lower than 0.5 were discarded. cropland $=21$ sites, deciduous forest $=20$ sites, evergreen forest $=24$ sites, grassland $=18$ sites, mixed forest $=5$ sites, shrubland $=4$ sites, and wetland $=12$ sites. 

Chapter 6

\section{General discussion}




\subsection{Introduction}

There is no doubt that the increase of atmospheric carbon dioxide $\left(\mathrm{CO}_{2}\right)$ concentration due to anthropogenic emissions is a key factor driving climate change (Andres et al., 1999; Canadell et al., 2007; Vitousek, 1997). As such, understanding the global carbon (C) cycle is at the forefront of policy debates and climate research. To implement effective C-related policies in the context of the United Nations Framework Convention on Climate Change (UNFCCC) agreements, accurate estimation of the biosphere-atmosphere $\mathrm{CO}_{2}$ exchange is required. However, such a task is not straight-forward due to the complexity of the C-climate feedback mechanisms; therefore there remain large uncertainties on the current estimates of land-atmosphere $\mathrm{CO}_{2}$ fluxes (Ciais et al., 2014a; Friedlingstein, 2015; Le Quéré et al., 2009). For instance, there is a need to better understand the ecosystem C-cycle mechanisms that are triggered by climate extremes and associated disturbances for obtaining more reliable estimates of the magnitude and even the sign of future C-cycle feedbacks. Scientists have been debating to what extent a proxy for disturbances history (i.e. forest age) controls the spatiotemporal variability of net $\mathrm{C}$ balance. While evidence of the role of forest age to the terrestrial net $\mathrm{C}$ fluxes were found, a debate on its relative importance remains. Additionally, climate and vegetation variability have been recognized to have widespread consequences on the $\mathrm{C}$ balance of terrestrial ecosystems (Reichstein et al., 2013). However, the responses of terrestrial ecosystem to the temporal dynamics of climate and vegetation are rather non-linear and complex (Bolt et al., 2018; Molen et al., 2011), therefore there is still a debate regarding the future impacts of climate variability and disturbances on the global C cycle (Frank et al., 2015).

This thesis addresses two related problems for modeling the spatiotemporal variability of the $\mathrm{CO}_{2}$ fluxes: site history and ecological memory (EM) effects; therefore contributes to provide insights of the role of ecosystem history in understanding the biosphere-atmosphere $\mathrm{CO}_{2}$ exchange dynamics. Overall, my thesis demonstrates the importance of ecosystem history for biosphere-atmosphere $\mathrm{CO}_{2}$ exchange. By showing the importance of forest age in explaining the spatiotemporal variability of net ecosystem production (NEP) at FLUXNET site level, I am able to provide a new perspective on the global forest $\mathrm{C}$ balance. Additionally, I show that deep learning (DL) methods are able to learn past climate and vegetation dynamics for improving $\mathrm{CO}_{2}$ flux estimates. This finding opens new avenues to explicitly quantify and represent EM effects in $\mathrm{C}$ cycle models. Yet my work also indicates that methodological and data characteristic challenges still persist. In the present chapter, I synthesise and discuss the main findings of my thesis. I also contrast my findings to research conducted by others and reflect on the limitations of the thesis. Finally, I provide some visions and future directions concerning new modeling frameworks to better capture terrestrial ecosystem dynamics and operational systems for $\mathrm{CO}_{2}$ flux monitoring. 


\subsection{Specific contributions of this thesis}

In the general introduction (Chapter 1), a series of research questions were defined. This section summarises the answers to these questions based on the analysis carried out in Chapter 2 to Chapter 5.

\section{What is the contribution of forest age to the terrestrial net $C$ fluxes?}

The first explored role of ecosystem history in biosphere-atmosphere $\mathrm{CO}_{2}$ exchange, presented in Chapter 2 and Chapter 3, is based on quantifying the relevance of disturbance history in the forest $\mathrm{C}$ balance. This is shown from two different perspectives: local and global. In the local analysis, I investigate whether a proxy for site history, that is forest age, contributes to explain the spatiotemporal variability of NEP at annual scale (Chapter 2). The relative importance of forest age in explaining NEP variability has previously been suggested at ecosystem-level. In fact, the early forest dynamics theory on net primary productivity (NPP) trajectories with forest age by Odum (1969) can be seen as a first hypothesis that such an effect could exist. Still, the quantification of the role of forest age in explaining the spatiotemporal variability of NEP is still under debate (Amiro et al., 2006; Fernández-Martínez et al., 2014; Gao et al., 2016). In this study, I find that including the forest age effects on NEP increases the model performance (Chapter 2) (Table 2.2). I also find that not including forest age in the statistical model leads to a substantial decrease in modeling efficiency, while removing proxies for environmental conditions leads to a minor decrease in explaining the variation of NEP. These findings confirm our understanding that forest age is an important driver of NEP spatiotemporal variability. It also suggests that forest age contains relevant ecological information not captured by other single variables and can be seen as a surrogate for several environmental drivers (e.g. nutrient availability, photosynthesis) that are more directly mechanistically coupled with $\mathrm{C}$ cycling processes.

\section{Can forest age be explicitly accounted for when upscaling net $\mathrm{CO}_{2}$ fluxes?}

The question that opens up after this work is to assess whether considering disturbance history for the upscaling of net $\mathrm{CO}_{2}$ flux data could provide more reasonable forest $\mathrm{C}$ balance estimates than current state-of-the-art global NEP products (Jung et al., 2011; Tramontana et al., 2016) (Chapter 3). More precisely, this global analysis describes a first attempt to include a proxy for disturbance history (i.e. forest age), when estimating global net forest $\mathrm{C}$ balance based on eddy-covariance (EC) data and a new forest age map (Fig. 3.1) as well as gross primary productivity (GPP) and climate global products (Chapter 3). This study provides not only new estimates of global forest NEP and net biome production (NBP) (Table 3.1), but also more insights on the location of forest $\mathrm{C}$ sinks (Fig. 3.5). The global estimates of forest NBP are found to be in agreement with independent forest inventory measurements, although substantial differences at biome level are observed. Yet, the results found in Chapter 3 are contingent on both the certainty of the forest age maps and on the representativeness of 
the FLUXNET sites in the environmental space. As a result, there is a need for producing more accurate forest age maps as well as for more FLUXNET sites with longer time series and higher data quality to corroborate these findings. It would be therefore relevant to contrast the conclusions of Chapter 3 with an analysis integrating the future releases of FLUXNET datasets that will present much more flux towers with longer time series. Generally, this work emphasizes the need for supporting networks that are collecting in-situ data (e.g. forest inventory data, EC data) to not only better understand regions poorly sampled but also to provide robustness in our conclusions with regard to ecosystem functioning. From a methodological perspective, Chapter 2 and Chapter 3 demonstrate both the advantages and limitations of empirical models based on EC data for answering questions about the forest $\mathrm{C}$ balance.

\section{How important is vegetation and climate's EM effects on terrestrial $\mathrm{CO}_{2}$ fluxes?}

The rest of the thesis turns its attention to understand the vegetation and climate's EM effects on terrestrial $\mathrm{CO}_{2}$ fluxes. Inspired from the fact that recurrent neural network (RNN) models have shown the potential to capture long-term temporal dependencies (Bahdanau et al., 2014; Hinton et al., 2012; Sutskever et al., 2014), Chapter 4 and Chapter 5 focus on the concept of EM effects by using dynamic statistical models, such as RNN. In Chapter 4, I test whether a Long-Short-Term Memory (LSTM) (i.e. a type of RNN model) model is able to learn temporal climate and vegetation dynamics to reproduce net $\mathrm{CO}_{2}$ flux dynamics more realistically across scales for forest and savanna ecosystems at 185 FLUXNET site level. I use time series of Landsat sensors since 1980 to integrate long-term (more than 30 years) vegetation dynamics (Fig. 4.3). The idea of this study was originally to capture long-term vegetation variabilities and disturbance regimes at FLUXNET site level for understanding how these temporal variations are reflected in the contemporary $\mathrm{CO}_{2}$ fluxes of forest and savanna ecosystems (Fig. 1.5). In this chapter, I could demonstrate the relevance of capturing vegetation and climate memory effects when predicting forest net ecosystem exchange (NEE) (Table 4.4 and Fig. 4.7). Furthermore, my assumption was that an LSTM model would have been able to better predict $\mathrm{CO}_{2}$ flux anomalies compared to commonly used non-dynamic methods (Jung et al., 2011; Tramontana et al., 2016). However, this thesis indicates a relatively poor predictive capacity for the NEE anomalies at seasonal and inter-annual scales (Fig. 4.4). Additionally, my hypothesis that the presented statistical model could better represent the $\mathrm{C}$ dynamics of young or recently disturbed forests could not be confirmed (Fig. 4.8). Overall, this analysis reveals that an LSTM model has a better capacity to learn the seasonal temporal contexts of vegetation and climate compared to a non-dynamic method (i.e. Random Forest (RF) model) or to an LSTM model constrained with input data having unrealistic temporal architecture (i.e. permutation experiment). I could also conclude that the better capacity of an LSTM model to learn the seasonal temporal context of vegetation and climate may explain its overall better capacity to predict the spatial variability in forest NEE (Table 4.4). 


\section{What is the magnitude and geographical distribution of climate and vegetation EM effects on biosphere-atmosphere $\mathrm{CO}_{2}$ exchange globally?}

Chapter 5 provides a global perspective with regard to vegetation and climate's EM effects on biosphere-atmosphere $\mathrm{CO}_{2}$ exchange. The results obtained in Chapter $\mathbf{5}$ show the magnitude and the geographical distribution of vegetation and climate's EM effects. This study shows that there are widespread memory effects across $\mathrm{CO}_{2}$ fluxes (Fig. 5.2), while some ecosystem and environmental characteristics (e.g. air temperature $\left(\mathrm{T}_{\text {air }}\right.$ ), vapor pressure deficit (VPD), global radiation ( $\mathrm{Rg}$ ), normalised difference vegetation index (NDVI), aboveground biomass (AGB)) control these observed spatial patterns (Fig. 5.5). Another interesting finding is that the EM effects observed are largely related to the seasonal vegetation and climate's variations and to a lower extent to inter-annual variability (Fig. 5.7). This study highlights that EM effects are most apparent for ecosystem respiration (ER) than for GPP (Fig. 5.A8). This finding are expected because soil $\mathrm{C}$ pools are key regulators of heterotrophic respiration ( $\mathrm{Rh}$ ) and the former are known to experience lag effects related to for example past land use or management (Ward et al., 2016). Yet, it is not clear how much information related to the changes in soil $\mathrm{C}$ pools is contained in the input variables, therefore EM effects observed in ER, hence NEE are very likely conservatives.

5. What are the implications of EM effects in net $\mathrm{CO}_{2}$ flux's responses during climate extreme events?

Additionally, I explore to what degree vegetation and climate's EM effects control the biosphere-atmosphere $\mathrm{CO}_{2}$ responses to a specific climate extreme event (i.e. 2018 European heatwave) (Fig. 5.6 and Fig. 5.A5). The differences between the anomalies inferred from an LSTM either containing EM effects or not provide insights on the contribution of these effects to the ecosystem response during a drought event. I am able to demonstrate that considering the temporal contexts of vegetation and climate dampens the response's magnitude (negative or positive) of an ecosystem during the heatwave (Fig. 5.6b). However, this analysis is done on a specific climate extreme event; therefore it would be relevant to perform a similar analysis for other reported climate extreme events (e.g. European heat wave in 2003, dry spells in the Amazon basin in 2005, forest fires in Russia in 2010, and so on) to see whether comparable patterns are found or not. Similarly, it would also be interesting to look at the years following any climate extreme events (e.g. the year 2019 for the 2018 European heatwave). This will provide knowledge on the lag effects of any climate extreme events in the following years as well as help us to understand what type of ecosystems are experiencing these lag effects. Yet, questions remain on whether the observed effects for the 2018 European heatwave were consistent with other observations related to this specific climate extreme event. The future releases of the FLUXNET datasets, containing $\mathrm{CO}_{2}$ flux observations for the year 2018 at European level, will be key for validating the observed anomalies in the global $\mathrm{CO}_{2}$ flux products presented in Chapter 5. 


\subsection{Reflection}

The following sections discuss the findings of this thesis, reflect on some of the limitations found in the thesis, and propose a series of avenues to overcome these limitations. Visions and future directions concerning $\mathrm{C}$ cycle studies and the understanding of the Earth System complement this general discussion.

\subsubsection{Evidence of the importance of forest age to the terrestrial net $\mathrm{CO}_{2}$ fluxes}

\section{What does forest age stand for?}

The thesis provides evidence for an effect of forest age on NEP spatiotemporal variability at annual scales. In Chapter 2, I observe from an updated EC dataset that forest age is the most important variable in explaining NEP spatiotemporal variability among a series of other biotic and abiotic factors (Table 2.2). The magnitude of the effects of forest age on annual forest $\mathrm{C}$ balance noted in Chapter 2 is considerable but is in contradiction with previous research (Fernández-Martínez et al., 2014). Instead of forest age, nutrient availability (NA) has been described as a key regulator for forest C balance (Bhatti et al., 2002; Fernández-Martínez et al., 2014; Janssens et al., 2010; Vicca et al., 2012). Yet in my work on quantifying the effect of forest age in the annual net forest carbon balance (Chapter 2), the NA of the forest ecosystems is not considered as an important driver for reproducing the spatiotemporal dynamics of NEP. It remains unclear why NA does not appear to be a strong determinant of NEP variability in Chapter 2 compared to previous studies. One could surmise that including forest age to some extent represents the nutrient status of a given forest ecosystem (Brais et al., 1995; Entry and Emmingham, 1995) and is a more accurate proxy for the nutrient status of a forest than the simple classification of NA used in this thesis (i.e. low, medium, and high NA). As a result, in spite of the absence of NA from the set of predictor variables selected by the feature selection algorithm (Kursa and Rudnicki, 2010) (Table 2.A3), a RF constrained by a combination of environmental variables and forest age could conceivably reproduce the nutrient status of forest ecosystems. Future research could target the link between forest structure, environmental conditions, and NA across different forest ecosystems and climatic regions to corroborate such a hypothesis.

Furthermore, the question of what $\mathrm{C}$ cycling mechanisms forest age is coupled with still remains. Although I am able to reproduce the spatial variability of forest age realistically from AGB and a series of climate variables (i.e. new forest age map in Chapter 3), understanding what forest age stands for when explaining the spatiotemporal variability of NEP is more challenging. One obvious assumption would be that the amount of $\mathrm{C}$ stored in a forest is closely related to the age-NEP spatiotemporal dynamics. In fact, since belowground and aboveground $\mathrm{C}$ stocks change with forest age (Fig. 1.4), it would not be too speculative to say that terrestrial C sink dynamics are controlled by a shift in forest age, therefore by changes in AGB. However, replacing forest 
age by a series of proxies for $\mathrm{C}$ stock estimates (i.e. AGB and growing stock volume (GSV)) does not achieve comparable predictive capacity as the original statistical model presented in Chapter 2 (Table 6.1). This could suggest that mean AGB and mean GSV are not good proxies for reproducing the effects of forest age on NEP, therefore other metrics, such as the differences between the potential AGB and the measured AGB, could be considered as a proxy for forest age. Additionally, looking at the belowground $\mathrm{C}$ stocks might also be an interesting avenue to explore for better representing both the respiratory processes (i.e. $\mathrm{Rh}$ and autotrophic respiration (Ra)) and the belowground allocation, which are known to be key regulators of the forest $\mathrm{C}$ balance (Ryan et al., 1997). Such missing processes could explain the high NEP residual values observed in Chapter 2, in particular for very young forests (Fig. 2.4). Consequently, there is a need to collect more measurements not only spatially but also temporally of AGB and belowground biomass (BGB) at FLUXNET site-level. The question of whether the effects of forest age on NEP are mainly driven by belowground processes, aboveground processes or both could be addressed this way.

Table 6.1: Model performance in explaining the spatiotemporal variability of NEP using the original model (Chapter 2), a model excluding forest age but including AGB, a model excluding forest age but including GSV. The (-) and (+) symbols mean that the predictive variable(s) were removed from or added to the RF models, respectively. These results were computed in a leave-one-siteout cross-validation mode. $\mathrm{R}^{2}$ = coefficient of determination; NSE = Nash-Sutcliffe model efficiency coefficient; $\mathrm{RMSE}=$ root mean squared error; $\mathrm{MAE}=$ mean absolute error; total $\mathrm{n}=716$ for site-years and $\mathrm{n}=126$ for site-average.

\begin{tabular}{|c|c|c|c|c|}
\hline & $\mathbf{R}^{2}$ & NSE & $\operatorname{RMSE}\left(\mathbf{g C ~ m}^{-2} \mathbf{y}^{-1}\right)$ & $\operatorname{MAE}\left(\mathbf{g C ~ m}^{-2} \mathbf{y}^{-1}\right)$ \\
\hline \multicolumn{5}{|l|}{ Site-years } \\
\hline original model & 0.62 & 0.62 & 180.14 & 131.09 \\
\hline$(-)$ age (+) AGB & 0.23 & 0.21 & 258.62 & 194.73 \\
\hline$(-)$ age (+) GSV & 0.23 & 0.21 & 257.25 & 195.92 \\
\hline \multicolumn{5}{|l|}{ Site-average } \\
\hline original model & 0.71 & 0.71 & 162.38 & 117.71 \\
\hline$(-)$ age $(+)$ AGB & 0.24 & 0.23 & 249.03 & 191.19 \\
\hline$(-)$ age $(+) \mathrm{GSV}$ & 0.29 & 0.29 & 239.74 & 184.49 \\
\hline
\end{tabular}

The need for better representing the age-spectrum in EC data

Demonstrating forest age as an important control of forest NEP is contingent on the distribution of the EC sites along the forest age spectrum. In fact, one of the main limitations of the dataset used in Chapter $\mathbf{2}$ and Chapter $\mathbf{3}$ is its biased sampling not only in the age-dimension but also in the environmental space. For instance, while young-to-intermediate forests are dominant in the FLUXNET datasets, very recently disturbed ( $<10$ years) and old-growth ( $>100$ years) forests are relatively under-represented (Fig. 2.A2). As such, the spatiotemporal forest age-NEP 
dynamics could not be properly described along the forest age spectrum among different climate zones and plant functional types (PFTs). Yet, the data-oriented character of the statistical model presented in Chapter 2 has the advantage of a purely empirical model, and thus has the ability to adapt to the data. Although this empirical model is based on a global EC dataset and makes use of a single global age-NEP relationship, the statistical model described in Chapter $\mathbf{2}$ appears to be able to adapt to local environmental conditions and to specific forest successional dynamics (Fig. 6.1). As such, the data-adaptive property of the presented statistical model may have overcome some of the limitations of the FLUXNET datasets with regard to the age-dimension representativeness. Still, my work advocates for the integration of more very young ( $<10$ years) and old ( $>100$ years) EC forest sites across different biomes and ecosystems to ascertain our current understanding of the link between forest age and the $\mathrm{C}$ balance.
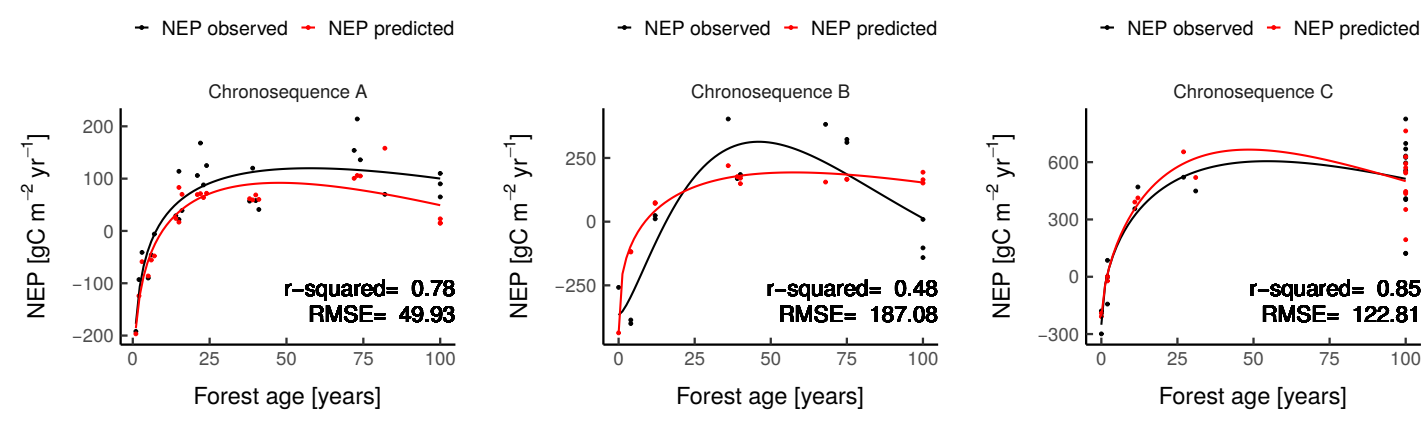

Figure 6.1: Reconstruction of independent chronosequences based on the statistical model presented in Chapter 2. Data of the chronosequences A and B were collected from boreal DBF and ENF ecosystems, respectively, while chronosequence $\mathrm{C}$ was a temperate $\mathrm{DBF}$ forest. The RF model used in Chapter 2 was used to predict NEP in these independent chronosequences by using the same set of variables. $\mathrm{NEP}=\mathrm{f}\left(\right.$ age $, \mathrm{f}($ Age $\left.), \mathrm{GPP}^{\prime}, \mathrm{T}_{\text {air }}, \mathrm{N}_{\text {deposition }}\right)$

\subsubsection{Limitations of the presented global forest net $\mathrm{C}$ balance estimates}

My thesis offers a novel look at the global patterns of forest NEP. Based on the findings of Chapter 2, I am able to translate a new forest age map, GPP, and environmental global products into a series of forest NEP global maps at $0.5^{\circ}$ spatial resolution representing the period 20012013. Chapter 3 illustrates the location and the magnitude of the forest $\mathrm{C}$ sinks. The results demonstrate that a large $\mathrm{C}$ sink dominated the young and productive temperate forests of Western Europe and the Eastern United States as well as the temperate and subtropical forests of China (Fig. 3.3). As this thesis shows, humid tropical forests are a small $\mathrm{C}$ sink that is geographically ubiquitous across the tropics. 


\section{Uncertainty of the global NEP and NBP estimates}

The latter finding is in contradiction with existing data-driven NEP products (Jung et al., 2011; Tramontana et al., 2016), which find a large $\mathrm{C}$ sink in the humid tropical forests. However, the sparse sampling of wet tropical regions is a particularly important problem for the upscaling exercise presented in Chapter $\mathbf{3}$ and could challenge my findings. The majority of included sites are localized in temperate ecosystems, while tropical ecosystems constitute only a small subset of sites (Fig. 2.A1). Tropical forests are known to be important ecosystems in the C cycle due to their high productivity and capacity to sequester a large amount of C (Pan et al., 2011a). As such, it is of relevance for developing global statistical data-driven models that are able to provide accurate predictions of forest $\mathrm{C}$ balance in tropical ecosystems. However, it is not clear how the limited number of tropical sites used in Chapter $\mathbf{3}$ affects the observed spatial patterns in this region (e.g. extrapolation uncertainty) and how confident one can be on the presented C balance of tropical ecosystems. My work thus underlines the necessity for more FLUXNET sites in tropical biomes and the maintenance of the existing FLUXNET sites. Long-term and better quality EC datasets in the tropics are indispensable to understand the global $\mathrm{C}$ cycle.

Additionally, Chapter 3 provides new estimates of global forest NEP and NBP (Table 3.1). As I note, the regional NEP budgets are rather similar between the presented approaches (i.e. AC-Amiro, AC-Tang, and Clim), while the NBP estimates are somehow in agreement with an independent forest inventory approach (Pan et al., 2011b). However, I identify substantial differences per biome between the presented approach and the Pan et al. (2011b) study, in particular in tropical and temperate regions. I propose a couple of arguments for explaining these differences, for example the Pan et al. (2011b) study possibly underestimates the soil C storage in temperate regions. As previously mentioned, the differences observed in the tropics are very contingent on the quality of the EC data in such an ecosystem, which called for being cautious in drawing conclusions from the results presented in Chapter 3. However, the observed spatial patterns (Fig. 3.3b) and the regional NEP estimates in the Amazon region somehow coincide with the findings provided by the forest gap model FORMIND constrained with forest structure (Rödig et al., 2018) in which they suggest a rather low C sink in the Amazon rainforest (i.e. 0.56 PgC year $\left.^{-1}\right)$.

Generally, I find it critical to emphasize that comparing regional estimates across methods can potentially be misleading, therefore such comparisons have to be interpreted very carefully. For instance, one can see that whether an approach considers the age effects on NEP or not can reach relatively similar conclusions with regard to regional C budgets (Table 3.1, AC-* approaches vs. Clim approach). However, there are widespread differences between the NEP spatial patterns of these two aforementioned approaches (i.e. AC-Amiro and AC-Clim) (Fig. 3.5), suggesting that the global forest $\mathrm{C}$ balance estimates of these two methods are, to a certain extent, similar simply because the observed differences in the spatial patterns are canceling each other out. Understanding the spatial patterns are therefore as important as providing global estimates in $\mathrm{C}$ cycle studies. 
The need for $\mathrm{CO}_{2}$ flux products with finer spatial resolution

By analyzing the global patterns of forest NEP (Chapter 3), it appears that a limited number forest ecosystems are a net $\mathrm{C}$ source across the globe (e.g. boreal forests, African dry forests) (Fig. 3.3). In contrast, Shugart et al. (2018) using a tree-based model shows widespread forest areas in the Eurasian boreal region to be $\mathrm{C}$ sources as well as in tropical regions undergoing disturbances. The question is then how to explain the discrepancies in the location of the $\mathrm{C}$ sources between the two different methods. One of the big limitations of the presented global forest NEP product is the coarse spatial resolution of the GPP products used in this study (i.e. $0.5^{\circ}$ spatial resolution). Thus, one of the reasons why the area of $\mathrm{C}$ source is relatively small could be that the forest age-GPP relationship (Tang et al., 2014) was challenged in Chapter 3 (Fig. 3.4a). It is known that the interactions between forest age and GPP control forest C dynamics (Odum, 1969; Tang et al., 2014) and having unrealistic age-GPP interactions in the globally gridded forcing datasets could strongly affect our forest $\mathrm{C}$ balance estimates. Therefore, the generation of spatially high-resolution datasets (e.g. $1 \mathrm{~km}$ spatial resolution) could provide a valuable source of information in order to avoid wrong or biased conclusions on the location of forest $\mathrm{C}$ sources and sinks globally.

\section{Challenges and opportunities to map forest demography}

Global forest age estimates introduce major uncertainties in the estimates of the global forest NEP. A sensitivity analysis demonstrates that depending on the forest age product used, the resulting forest NEP spatial patterns can be substantially different (Fig. 3.A8). These observed differences in the spatial patterns suggest that the conclusions presented in Chapter $\mathbf{3}$ are also limited by the certainty of the forest age products. By considering different AGB maps (i.e. European Space Agency (ESA) GlobBiomass product corrected for different tree cover thresholds) to produce a series of global forest age maps (Fig. 3.A5), I could account for the uncertainty related to forest age when assessing the forest $\mathrm{C}$ balance estimates. Still, it is rather challenging to obtain accurate estimates of forest demography at the global scale, therefore the spatial patterns of forest age remain uncertain. For example, there is biased and incomplete global spatial coverage of the forest inventory databases (Álvarez-Dávila et al., 2017; Anderson-Teixeira et al., 2018; Anderson-Teixeira et al., 2016; Baker et al., 2016; Johnson et al., 2016; Lewis et al., 2013; Mitchard et al., 2014; N'Guessan et al., 2019; Poorter et al., 2016; Schepaschenko et al., 2017; Somogyi et al., 2008; Sullivan et al., 2017) with the pan-tropical regions largely under-represented compared to the temperate forest ecosystems (Fig. 3.A3). Consequently, this representativeness issue is expected to have an impact on the capacity of a statistical model to predict forest age estimates in these under-represented regions based on forest inventory data (Fig. 3.A4). As such, initiatives (e.g. ForestPlots.net initiative) should be supported so that they can continue their work in collecting forest inventory measurements in tropical forests. Increasing the number of forest inventory plots in the tropics could contribute to a better understanding of 
tropical forests' behavior. Furthermore, very few regional or global forest age maps (Chazdon et al., 2016; Loboda and Chen, 2017; Moreno et al., 2017; Pan et al., 2011a; Poulter et al., 2019; Zhang et al., 2017) are currently available, which hinders the validation of any attempts for representing the forest demography. Yet, the comparison between the different regional and global forest age products carried out in Chapter 3 depicts substantial discrepancies and confirms the challenges for mapping forest age (Fig. 3.A6). In this context, I would argue that one would need to create a set of ensembles from the different regional/global forest age maps in order to properly assess the uncertainties related to forest age of the global forest NEP spatial patterns. Alternatively, the creation of several forest age maps (following the approach developed in Chapter 3) using a series of independent global AGB products (e.g. ESA GlobBiomass product, forthcoming AGB global product from the Saatchi group) together with different tree cover thresholds' corrections could also provide both a valuable and consistent option for not only describing uncertainties in the forest age estimates but also in the global forest NEP. New remote sensing missions such as the National Aeronautics and Space Administration (NASA) Global Ecosystem Dynamics Investigation (GEDI) are also aiming to provide measurements of forest structure, therefore could be a relevant source of information to characterize forest demography and address $\mathrm{C}$ cycle questions.

\subsubsection{On the ability to capture $\mathrm{CO}_{2}$ flux anomalies}

Chapter 4 and Chapter 5 show that the use of a dynamic DL method (i.e. Long-Short-Term Memory (LSTM) model) could capture climate and vegetation's EM effects to reproduce $\mathrm{CO}_{2}$ flux dynamics across scales more realistically, particularly the seasonal variations. However, this thesis indicates a relatively poor predictive capacity for the NEE anomalies at seasonal and inter-annual scales (Fig. 4.4). It is not entirely clear why an LSTM model could not capture the anomalies in the $\mathrm{CO}_{2}$ fluxes at seasonal and inter-annual scales, although several hypotheses can be drawn.

\section{Temporal scales during the training procedure}

One of the main limitations of the method presented in Chapter 4 is the temporal resolution used during the training procedure of the LSTM models (i.e. monthly scale). Due to the temporal resolution of the Landsat data (i.e. 16-days) and the irregular character of the Landsat timeseries, I aggregate the input (i.e. Landsat bands and climate variables) and target (i.e. NEE) variables into monthly averages. However, for instance, the drought impacts on the functioning of vegetation state is generally observed at sub-monthly temporal resolution (Stocker et al., 2019; Vicca et al., 2016). Consequently, I would argue that any drought effects on the $\mathrm{CO}_{2}$ fluxes occurring during the observational period of EC data are mitigated at monthly scale, which may impede an LSTM model to fully learn the variations of vegetation in response to drought. Assuming that climate extreme events exert a strong control on the $\mathrm{CO}_{2}$ flux anomalies 
(Bastos et al., 2014; Ciais et al., 2005; Reichstein et al., 2007a), it would be interesting to apply such dynamic statistical model at weekly or even daily scale to better understand the effects of climate's temporal variabilities on $\mathrm{CO}_{2}$ flux responses.

\section{Limited number of disturbance events in the FLUXNET networks}

The properties of an LSTM model suggest a priori that such an approach would be able to capture features related to disturbance regimes (e.g. time since last disturbance, magnitude of the disturbance, speed of forest recovery) (Fig. 1.5) from long-term Landsat time-series, therefore help to reproduce anomalies in NEE. However, the sparse sampling of recently disturbed forests in the FLUXNET datasets is thus a particularly important problem. In fact, we report a very limited number of FLUXNET sites, which experienced disturbance events during the period of EC data collection or within the Landsat era. As a consequence, this can limit an LSTM in learning specific vegetation responses to disturbance regimes as well as their short- to long-term effects. This issue is further emphasized by the fact that both space and time were considered during the training procedure. Surprisingly, adding forest age as an input variable in the LSTM model does not improve its predictive capacity, in particular across FLUXNET sites (Fig. 4.8). This finding is somehow in contradiction with the results presented in Chapter 2, but could suggest that different variables/mechanisms are controlling $\mathrm{CO}_{2}$ fluxes across temporal scales. This raises the issue that the effects of site history, such as forest age, in controlling the spatiotemporal variability of annual NEP (Chapter 2) were not captured during the training procedure, which was performed at monthly scale. Assuming that most of the seasonal patterns of $\mathrm{CO}_{2}$ fluxes are controlled by climate seasonal variability and phenology, the opportunities of an LSTM model to learn the effects of other variables (e.g. forest age) are very likely limited, therefore the latter effects cannot be reflected across the different temporal scales (i.e. seasonal to inter-annual scales). Constraining the presented statistical models with cost functions computed at monthly, annual, and spatial scales could supply a way to overcome this problem.

\section{The application of LSTM models to synthetic data}

The fact that the presented LSTM model struggles to represent $\mathrm{CO}_{2}$ flux anomalies prompts the question of whether the architecture of the presented LSTM model is suitable to learn the different components of the vegetation and climate temporal context. In fact, the architecture of the LSTM model used in Chapter 4 is rather simple: a single-layered LSTM using a grid search for optimizing the hyperparameters (i.e. learning rate, number of hidden neurons, and dropout). It is, therefore, challenging to conclude to what degree the NEE residuals observed across scales is related to the complexity of the LSTM model, the characteristics of the Landsat data (e.g. noisy signals and high fraction of gaps in the time series), the temporal scale of the model training procedure (i.e. monthly scale), or even the properties of the EC data (e.g. quality, length of the time series). In fact, I still find puzzling that any attempts to reproduce $\mathrm{CO}_{2}$ flux anomalies 
with data-driven statistical models (Chapter 4, Tramontana et al. (2016), and Jung et al. (2011)) have not been yet successfull. Conversely, realistically reproducing the anomalies of the energy fluxes (i.e. sensible heat, latent heat, and net radiation) using a similar set of predictors have been achieved (Tramontana et al., 2016). In my opinion, this finding questions the quality of the EC data for $\mathrm{CO}_{2}$ flux measurements with regard to the properties of $\mathrm{CO}_{2}$ flux anomalies captured by EC method. In this context, the use of long-term $\mathrm{CO}_{2}$ flux synthetic data (i.e. noise free, long-term, and high temporal resolution data) derived from a prognostic process-based biosphere model (e.g. Carnegie Ames Stanford Approach (CASA) model (Field et al., 1995; Potter et al., 1993)) could be an interesting exercise to make a comprehensive assessment of the real capacity of an LSTM model to characteristize vegetation and climate's EM effects as well as to get some insights on the data quality of $\mathrm{CO}_{2}$ fluxes at FLUXNET sites when measuring the anomaly signals.

\subsubsection{Extrapolation uncertainties and the potential of transfer learning}

In Chapter 5, I find that areas with moderate $\mathrm{CO}_{2}$ inter-annual variability have the strongest EM effects (Fig. 5.A5), while semi-arid regions are expected to have relatively strong EM effects due to their high inter-annual variability of C uptake (Ahlström et al., 2015; Poulter et al., 2014). However, the fact that limited EM effects are found in semi-arid regions could also be related to the representativeness of these regions in the FLUXNET datasets. Similar to tropical sites, EC sites in semi-arid regions are under-represented in the FLUXNET datasets, which could hinder data-driven statistical models, such as an LSTM model, to properly capture the $\mathrm{CO}_{2}$ flux dynamics in these areas.

\section{Uncertainties in extrapolation due to representativeness}

The under-representation of specific environmental conditions (e.g. semi-arid regions, the tropics) in the FLUXNET datasets brings me to discuss critiques with regard to upscaling $\mathrm{CO}_{2}$ fluxes using FLUXNET datasets due the representativeness of the EC sites (Kumar et al., 2016; Schimel et al., 2015). However, there is currently no comprehensive assessment of extrapolation uncertainty presented in the FLUXNET datasets. It is therefore important to diagnose extrapolation uncertainty spatially and temporally for different $\mathrm{CO}_{2}$ fluxes to understand the strengths and weaknesses of such global $\mathrm{CO}_{2}$ flux products. The FLUXCOM initiative (http: //www.fluxcom.org/) is currently considering to provide a diagnosis of these extrapolation uncertainty issues (Fig. 6.2). Their approach is particularly interesting because it reflects distances in the environmental predictor space but not in the geographical space. While one would expect to observe a lot of extrapolation uncertainties in the wet tropics, hotspots in uncertainties are actually localized in semi-arid regions. These preliminary results could suggest the need for more EC sites in semi-arid regions, in particular, when one considers their future role in the C cycle (Giorgi, 2006; Hoerling et al., 2011; Sheffield and Wood, 2008). Furthermore, due 
to the limited number of tropical sites, it still remains difficult to draw any conclusions on whether one would need more data in the tropics or not: the problem of the unknown unknowns.
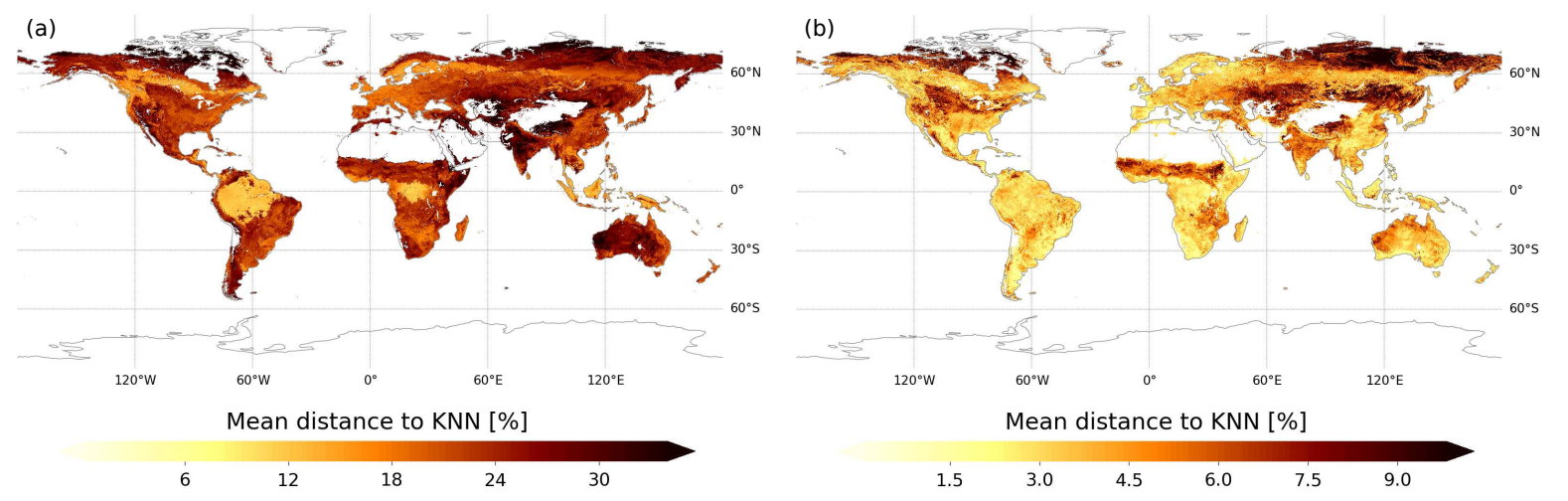

Figure 6.2: Spatial patterns of extrapolation uncertainty for GPP in the context of the FLUXCOM framework. Annual mean (a) and annual standard deviation (b) of the mean distance to k nearest neighbors (KNN) in training data for the year 2015 are shown. The distance estimates are normalized, that is a value of $\mathrm{X} \%$ means that one would expect an error increase of $\mathrm{X} \%$ error with regard to having observations for these conditions. In other words, this metric represents the error increase when one goes further away from the training data. Data provided by Martin Jung.

The potential of transfer learning for upscaling $\mathrm{CO}_{2}$ fluxes

The problem of representativeness is difficult to tackle, although some solutions exist. Increasing the number of FLUXNET sites in the under-represented regions is part of the solution. However, a more cost-effective avenue is the use of transfer learning, where a model trained on one task is re-purposed on a second related task. The application of transfer learning when modeling $\mathrm{CO}_{2}$ fluxes can be done by for example taking advantage of global retrievals of satellite-based sun-induced chlorophyll fluorescence (SIF) (e.g. GOME-2 (Joiner et al., 2013; Köhler et al., 2015), TROPOMI-SIF (Köhler et al., 2018)), particularly for GPP. In fact, several studies have found strong positive seasonal correlations between SIF and GPP (Frankenberg et al., 2011; Guanter et al., 2014; Joiner et al., 2014; Lee et al., 2013; Parazoo et al., 2014). The observed seasonal correlations between satellite-based SIF and GPP motivate the use of transfer learning. By following the idea of Junzhi Liu, one can train an LSTM model on satellite-based SIF, re-use this pre-trained model to learn GPP dynamics at FLUXNET site level, and further simulate GPP globally (Fig. 6.3). In selected regions where extrapolation uncertainties are usually an issue (Fig. 6.2), it appears that transfer learning can really help in overcoming issues related to extrapolation (i.e. LSTM vs. LSTM+transfer learning). More precisely, the comparison between LSTM and LSTM+transfer learning reveals that the latter can better reproduce both the course of the SIF annual cycle and its magnitude. Although the presented results are preliminary and more investigation has to be done, the work of Junzhi Liu on transfer learning technique is conceptually very promising for upscaling $\mathrm{CO}_{2}$ fluxes and overcoming extrapolation uncertainties. In the 
context of Chapter 5, the observed patterns of vegetation and climate's EM effects are contingent both on the spatiotemporal signals presented in the global forcings (i.e. vegetation state and climate variables) and on the features learned by the LSTM at FLUXNET site level. As such, the application of transfer learning could enable an LSTM to capture additional features not contained in the EC observations, thereby may result in higher EM effects magnitude and reveal new regions showing substantial EM effects.

$$
\text { - LSTM - LSTM+transfer learning - SIF }
$$
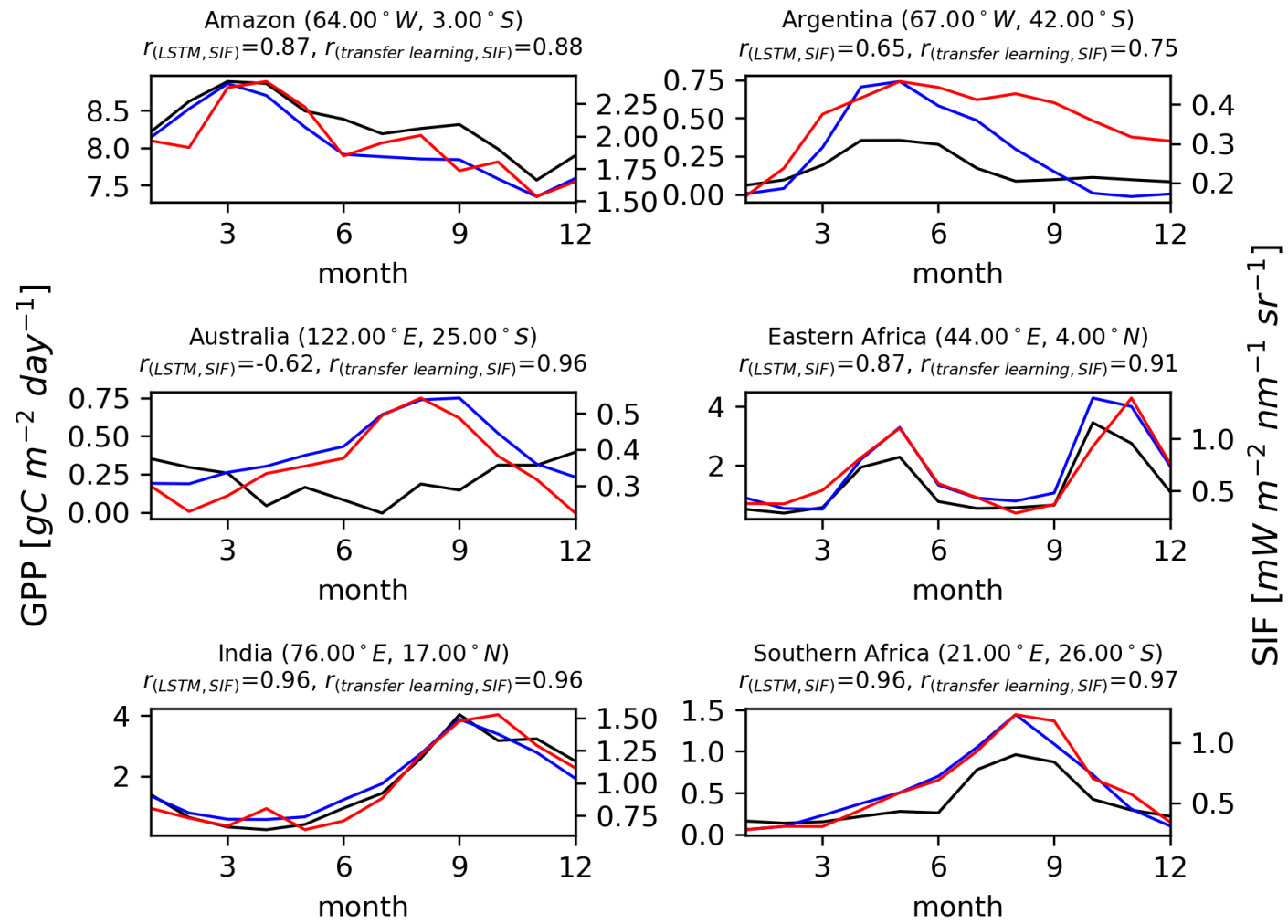

Figure 6.3: Mean seasonal cycle of GPP estimated from an LSTM model only trained at FLUXNET site level (i.e. LSTM), GPP esimtated from an LSTM model trained both on global satellite-based SIF and FLUXNET site (i.e. LSTM+transfer learning), and SIF retrieved from GOME-2 (i.e. SIF). Line plots depict the mean seasonal cycle of either GPP (black and blue lines for LSTM and LSTM+transfer learning, respectively) or SIF (red lines) for selected locations (median values within a $0.5^{\circ}$ bounding box around selected locations). Figure was derived by following the idea of Junzhi Liu.

\subsubsection{The integration of multiple data sources for $\mathrm{CO}_{2}$ flux estimates}

While the chosen predictors in Chapter $\mathbf{4}$ and Chapter 5 reflect many climate and land surface properties in great detail, they also omit important relevant ecosystem characteristics such 
as species richness (Musavi et al., 2017), information on the spatial context (e.g. landscape patchiness, fractal dimension) (Premke et al., 2016), soil moisture (Stocker et al., 2019), or possibly changes in $\mathrm{C}$ stocks that can also control the ecosystem functioning and that relate to vegetation and climate's EM effects. In Chapter $\mathbf{4}$ and Chapter 5, I use a single type of satellite dataset as a proxy for vegetation state, that is optical remote sensing data (Landsat and MODerate-resolution Imaging Spectroradiometer (MODIS) data). The use of only one type of sensor can result in biased conclusions about the performance of any data-driven statistical models, and thus about the functioning of ecosystems globally. For instance, the use of Synthetic Aperture Radar (SAR) images have been shown to be a good proxy for temporal changes of surface soil moisture (Bauer-Marschallinger et al., 2019), while soil moisture exerts a strong control on photosynthesis and vegetation primary production (Stocker et al., 2019). As a result, the integration of such datasets representing soil moisture conditions either at coarse (e.g. ESA climate change initiative (CCI) soil moisture product) or fine (e.g. Sentinel-1 data) spatial resolution into $\mathrm{CO}_{2}$ flux data-driven models seems primordial. Furthermore, a series of initiatives are currently working on providing satellite-derived biomass annual maps by combining different sensors (e.g. ASCAT, Envisat ASAR, and ALOS PALSAR). These new products could potentially allow us to estimate net changes in vegetation $\mathrm{C}$ stocks during the last 30 years and therefore to infer environmental mechanisms behind such changes. Consequently, future efforts within the scientific community should aim to integrate not only remote sensing data related to phenology and physiology but also to changes in soil moisture conditions and $\mathrm{C}$ stocks when modeling the spatiotemporal variabilities of $\mathrm{CO}_{2}$ fluxes. Therefore, the integration of remote sensing products describing temporal changes of $\mathrm{C}$ stocks and soil moisture into existing statistical modeling frameworks should be prioritized in studies aiming to have a comprehensive assessment of vegetation and climate's EM effects, thereby a better understanding of the C cycle.

\subsection{Future prospects}

\subsubsection{Potential of hybrid modeling for Earth System sciences}

Machine learning approaches have demonstrated their great capacity to provide answers to geoscience and remote sensing questions and have become universal tools in Earth System sciences. The main limitation of the methods is that they cannot integrate existing knowledge about natural processes and it is difficult to extract interpretable information from these methods ('black box' concept). While the link between physical modeling and machine learning methods have been often overlooked in the past, the synergy between the two approaches has recently gained attention (Camps-Valls et al., 2018; Karpatne et al., 2017a; Karpatne et al., 2017b). Integrating data-driven approaches into physical models could be relevant for optimizing the parameters required in physical models. Instead of being static, these parameters could be more 
dynamic, interdependent, and contextual when being learned from appropriate sets of statistical covariates (Reichstein et al., 2019). Additionally, the interpretability property of the physical models could help us in understanding the statistical interactions between co-variates being learned by machine learning methods. Some of the relevant relevant biogeochemical processes presented in this thesis are difficult to emulate in machine learning. As such, combining fully data-adaptive machine learning methods and physical models that obey established physical principles could provide a better understanding of the Earth system, but also for testing new hypotheses.

\subsubsection{Towards a near-real time monitoring of the $\mathrm{CO}_{2}$ fluxes}

The development of an alert system for near-real-time deforestation monitoring (www . globalforestwatch.org) paved the way to transfer technological innovation to an operational system. As a result, it is currently rather straight-forward for anyone to have access to tools for visualizing and understanding information that have large impacts in terms of public awareness about contemporary environmental issues. With the success of operational remote sensing based deforestation mapping, the question is then whether a similar operational system could be implemented for near-real-time monitoring of the $\mathrm{CO}_{2}$ fluxes. While producing a global product of $\mathrm{CO}_{2}$ fluxes in an operational way that is regularly updated would be desirable and highly beneficial for environmental conservation causes, the complexity of climate-carbon feedback mechanisms opens constantly new scientific questions. Therefore, the current unanswered questions on the climate-carbon feedback mechanisms could hinder $\mathrm{CO}_{2}$ flux monitoring to achieve the same operational level for deforestation monitoring. In addition, the creation of accurate maps for monitoring $\mathrm{CO}_{2}$ fluxes requires a plethora of input variables and complex data-driven models in order to reproduce $\mathrm{CO}_{2}$ flux observations realistically. Although our capacity to store a large amount of data and to use high-performance computers could overcome this problem, there are still some hurdles to provide a fully operational, near-real-time monitoring system for $\mathrm{CO}_{2}$ fluxes and discussion among the different stakeholders (e.g. remote sensing community, carbon cycle community, computer scientists, and policymakers/governments) should take place to better articulate how such an operational system could be set-up, whether it is really needed, and who is responsible for what. Alternatively, a series of initiatives are currently discussing the possibility of releasing annual updates of global $\mathrm{CO}_{2}$ and energy flux products, which could be seen as a step forward towards an operational system comparable to the one for deforestation monitoring. However, questions related to who is responsible for the maintenance of such an operational system or who stores the data would need to be answered before moving forward. 



\section{References}

Ahlström, A., M. R. Raupach, G. Schurgers, B. Smith, A. Arneth, M. Jung, M. Reichstein, J. G. Canadell, P. Friedlingstein, A. K. Jain, E. Kato, B. Poulter, S. Sitch, B. D. Stocker, N. Viovy, Y. P. Wang, A. Wiltshire, S. Zaehle, and N. Zeng (2015). "The dominant role of semi-arid ecosystems in the trend and variability of the land CO2 sink". Science 348.6237, 895-899.

Álvarez-Dávila, E., L. Cayuela, S. González-Caro, A. M. Aldana, P. R. Stevenson, O. Phillips, Á. Cogollo, M. C. Peñuela, P. v. Hildebrand, E. Jiménez, O. Melo, A. C. Londoño-Vega, I. Mendoza, O. Velásquez, F. Fernández, M. Serna, C. Velázquez-Rua, D. Benítez, and J. M. Rey-Benayas (2017). "Forest biomass density across large climate gradients in northern South America is related to water availability but not with temperature". PLOS ONE 12.3, e0171072.

Amiro, B. D., A. G. Barr, J. G. Barr, T. A. Black, R. Bracho, M. Brown, J. Chen, K. L. Clark, K. J. Davis, A. R. Desai, S. Dore, V. Engel, J. D. Fuentes, A. H. Goldstein, M. L. Goulden, T. E. Kolb, M. B. Lavigne, B. E. Law, H. A. Margolis, T. Martin, J. H. McCaughey, L. Misson, M. Montes-Helu, A. Noormets, J. T. Randerson, G. Starr, and J. Xiao (2010). "Ecosystem carbon dioxide fluxes after disturbance in forests of North America". Journal of Geophysical Research: Biogeosciences 115.G4.

Amiro, B. D., A. G. Barr, T. A. Black, H. Iwashita, N. Kljun, J. H. McCaughey, K. Morgenstern, S. Murayama, Z. Nesic, A. L. Orchansky, and N. Saigusa (2006). "Carbon, energy and water fluxes at mature and disturbed forest sites, Saskatchewan, Canada". Agricultural and Forest Meteorology 136.3, 237-251.

Anderson-Teixeira, K. J., A. D. Miller, J. E. Mohan, T. W. Hudiburg, B. D. Duval, and E. H. DeLucia (2013). "Altered dynamics of forest recovery under a changing climate". Global Change Biology 19.7, 2001-2021.

Anderson-Teixeira, K. J., M. M. H. Wang, J. C. McGarvey, V. Herrmann, A. J. Tepley, B. BondLamberty, and D. S. LeBauer (2018). "ForC: a global database of forest carbon stocks and fluxes". Ecology 99.6, 1507.

Anderson-Teixeira, K. J., M. M. H. Wang, J. C. McGarvey, and D. S. LeBauer (2016). "Carbon dynamics of mature and regrowth tropical forests derived from a pantropical database (TropForC-db)". Global Change Biology 22.5, 1690-1709. 
Andres, R. J., D. J. Fielding, G. Marland, T. A. Boden, N. Kumar, and A. T. Kearney (1999). “Carbon dioxide emissions from fossil-fuel use, 1751-1950". Tellus B 51.4, 759-765.

Aragão, L. E. O. C., L. O. Anderson, M. G. Fonseca, T. M. Rosan, L. B. Vedovato, F. H. Wagner, C. V. J. Silva, C. H. L. S. Junior, E. Arai, A. P. Aguiar, J. Barlow, E. Berenguer, M. N. Deeter, L. G. Domingues, L. Gatti, M. Gloor, Y. Malhi, J. A. Marengo, J. B. Miller, O. L. Phillips, and S. Saatchi (2018). "21st Century drought-related fires counteract the decline of Amazon deforestation carbon emissions". Nature Communications 9.1, 1-12.

Araújo, A. C., A. D. Nobre, B. Kruijt, J. A. Elbers, R. Dallarosa, P. Stefani, C. v. Randow, A. O. Manzi, A. D. Culf, J. H. C. Gash, R. Valentini, and P. Kabat (2002). "Comparative measurements of carbon dioxide fluxes from two nearby towers in a central Amazonian rainforest: The Manaus LBA site". Journal of Geophysical Research: Atmospheres 107 (D20), LBA 58-1-LBA 58-20.

Aubinet, M., Q. Hurdebise, H. Chopin, A. Debacq, A. De Ligne, B. Heinesch, T. Manise, and C. Vincke (2018). "Inter-annual variability of Net Ecosystem Productivity for a temperate mixed forest: A predominance of carry-over effects?" Agricultural and Forest Meteorology 262, 340-353.

Bahdanau, D., K. Cho, and Y. Bengio (2014). "Neural Machine Translation by Jointly Learning to Align and Translate". arXiv:1409.0473.

Baker, T. R., D. M. V. Díaz, V. C. Moscoso, G. Navarro, A. Monteagudo, R. Pinto, K. Cangani, N. M. Fyllas, G. L. Gonzalez, W. F. Laurance, S. L. Lewis, J. Lloyd, H. t. Steege, J. W. Terborgh, and O. L. Phillips (2016). "Consistent, small effects of treefall disturbances on the composition and diversity of four Amazonian forests". Journal of Ecology 104.2, 497-506.

Baldocchi, D. (2008). “'Breathing' of the terrestrial biosphere: lessons learned from a global network of carbon dioxide flux measurement systems". Australian Journal of Botany 56.1, 1-26.

Baldocchi, D., E. Falge, L. Gu, R. Olson, D. Hollinger, S. Running, P. Anthoni, C. Bernhofer, K. Davis, R. Evans, J. Fuentes, A. Goldstein, G. Katul, B. Law, X. Lee, Y. Malhi, T. Meyers, W. Munger, W. Oechel, K. T. Paw U, K. Pilegaard, H. P. Schmid, R. Valentini, S. Verma, T. Vesala, K. Wilson, and S. Wofsy (2001). "FLUXNET: A New Tool to Study the Temporal and Spatial Variability of Ecosystem-Scale Carbon Dioxide, Water Vapor, and Energy Flux Densities". Bulletin of the American Meteorological Society 82.11, 2415-2434.

Bastos, A., C. M. Gouveia, R. M. Trigo, and S. W. Running (2014). "Analysing the spatiotemporal impacts of the 2003 and 2010 extreme heatwaves on plant productivity in Europe". Biogeosciences 11.13, 3421-3435.

Bastos, A., P. Friedlingstein, S. Sitch, C. Chen, A. Mialon, J.-P. Wigneron, V. K. Arora, P. R. Briggs, J. G. Canadell, P. Ciais, F. Chevallier, L. Cheng, C. Delire, V. Haverd, A. K. Jain, F. Joos, E. Kato, S. Lienert, D. Lombardozzi, J. R. Melton, R. Myneni, J. E. M. S. Nabel, J. Pongratz, B. Poulter, C. Rödenbeck, R. Séférian, H. Tian, C. van Eck, N. Viovy, N. Vuichard, A. P. Walker, A. Wiltshire, J. Yang, S. Zaehle, N. Zeng, and D. Zhu (2018). "Impact of the 
2015/2016 El Niño on the terrestrial carbon cycle constrained by bottom-up and top-down approaches". Philosophical Transactions of the Royal Society B: Biological Sciences 373.1760, 20170304.

Bauer-Marschallinger, B., V. Freeman, S. Cao, C. Paulik, S. Schaufler, T. Stachl, S. Modanesi, C. Massari, L. Ciabatta, L. Brocca, and W. Wagner (2019). "Toward Global Soil Moisture Monitoring With Sentinel-1: Harnessing Assets and Overcoming Obstacles". IEEE Transactions on Geoscience and Remote Sensing 57.1, 520-539.

Becknell, J. M., A. R. Desai, M. C. Dietze, C. A. Schultz, G. Starr, P. A. Duffy, J. F. Franklin, A. Pourmokhtarian, J. Hall, P. C. Stoy, M. W. Binford, L. R. Boring, and C. L. Staudhammer (2015). "Assessing Interactions Among Changing Climate, Management, and Disturbance in Forests: A Macrosystems Approach”. BioScience 65.3, 263-274.

Beer, C., M. Reichstein, E. Tomelleri, P. Ciais, M. Jung, N. Carvalhais, C. Rödenbeck, M. A. Arain, D. Baldocchi, G. B. Bonan, A. Bondeau, A. Cescatti, G. Lasslop, A. Lindroth, M. Lomas, S. Luyssaert, H. Margolis, K. W. Oleson, O. Roupsard, E. Veenendaal, N. Viovy, C. Williams, F. I. Woodward, and D. Papale (2010). "Terrestrial Gross Carbon Dioxide Uptake: Global Distribution and Covariation with Climate". Science 329.5993, 834-838.

Beringer, J., L. B. Hutley, N. J. Tapper, and L. A. Cernusak (2007). "Savanna fires and their impact on net ecosystem productivity in North Australia”. Global Change Biology 13.5, 9901004.

Besnard, S., N. Carvalhais, M. A. Arain, A. Black, B. Brede, N. Buchmann, J. Chen, J. G. P. W. Clevers, L. P. Dutrieux, F. Gans, M. Herold, M. Jung, Y. Kosugi, A. Knohl, B. E. Law, E. Paul-Limoges, A. Lohila, L. Merbold, O. Roupsard, R. Valentini, S. Wolf, X. Zhang, and M. Reichstein (2019a). "Memory effects of climate and vegetation affecting net ecosystem $\mathrm{CO}_{2}$ fluxes in global forests". PLOS ONE 14.2, e0211510.

Besnard, S., N. Carvalhais, M. A. Arain, A. Black, S. d. Bruin, N. Buchmann, A. Cescatti, J. Chen, J. G. P. W. Clevers, A. R. Desai, C. M. Gough, K. Havrankova, M. Herold, L. Hörtnagl, M. Jung, A. Knohl, B. Kruijt, L. Krupkova, B. E. Law, A. Lindroth, A. Noormets, O. Roupsard, R. Steinbrecher, A. Varlagin, C. Vincke, and M. Reichstein (2018). "Quantifying the effect of forest age in annual net forest carbon balance". Environmental Research Letters 13.12, 124018 .

Besnard, S., N. Carvalhais, J. G. P. W. Clevers, F. Gans, M. Herold, M. Jung, U. Weber, and M. Reichstein (2019b). "Dynamic and lag effects of climate and vegetation on biosphereatmosphere $\mathrm{CO}_{2}$ exchange: a global analysis". In preparation.

Bhatti, J. S., M. J. Apps, and H. Jiang (2002). "Influence of nutrients, disturbances and site conditions on carbon stocks along a boreal forest transect in central Canada". Plant and Soil 242.1, 1-14.

Birdsey, R., K. Pregitzer, and A. Lucier (2006). "Forest carbon management in the United States: 1600-2100”. Journal of Environmental Quality 35.4, 1461-1469. 
Bodesheim, P., M. Jung, F. Gans, M. D. Mahecha, and M. Reichstein (2018). "Upscaled diurnal cycles of land-atmosphere fluxes: a new global half-hourly data product". Earth System Science Data 10.3, 1327-1365.

Bohnert, H. J., D. E. Nelson, and R. G. Jensen (1995). “Adaptations to Environmental Stresses.” The Plant Cell 7.7, 1099-1111.

Bolt, B. v. d., E. H. v. Nes, S. Bathiany, M. E. Vollebregt, and M. Scheffer (2018). "Climate reddening increases the chance of critical transitions". Nature Climate Change 8.6, 478.

Bonan, G. B. (2008). "Forests and Climate Change: Forcings, Feedbacks, and the Climate Benefits of Forests". Science 320.5882, 1444-1449.

Bowman, D. M. J. S., J. K. Balch, P. Artaxo, W. J. Bond, J. M. Carlson, M. A. Cochrane, C. M. D’Antonio, R. S. DeFries, J. C. Doyle, S. P. Harrison, F. H. Johnston, J. E. Keeley, M. A. Krawchuk, C. A. Kull, J. B. Marston, M. A. Moritz, I. C. Prentice, C. I. Roos, A. C. Scott, T. W. Swetnam, G. R. v. d. Werf, and S. J. Pyne (2009). "Fire in the Earth System". Science 324.5926, 481-484.

Brais, S., C. Camiré, Y. Bergeron, and D. Paré (1995). “Changes in nutrient availability and forest floor characteristics in relation to stand age and forest composition in the southern part of the boreal forest of northwestern Quebec". Forest Ecology and Management 76.1, 181-189.

Breiman, L. (2001). "Random Forests". Machine Learning 45.1, 5-32.

Buchmann, N. and E.-D. Schulze (1999). "Net CO2 and H2O fluxes of terrestrial ecosystems". Global Biogeochemical Cycles 13.3, 751-760.

Buermann, W., M. Forkel, M. O’Sullivan, S. Sitch, P. Friedlingstein, V. Haverd, A. K. Jain, E. Kato, M. Kautz, S. Lienert, D. Lombardozzi, J. E. M. S. Nabel, H. Tian, A. J. Wiltshire, D. Zhu, W. K. Smith, and A. D. Richardson (2018). "Widespread seasonal compensation effects of spring warming on northern plant productivity”. Nature 562.7725, 110.

Buitenwerf, R., B. Sandel, S. Normand, A. Mimet, and J.-C. Svenning (2018). "Land surface greening suggests vigorous woody regrowth throughout European semi-natural vegetation". Global Change Biology 24.12, 5789-5801.

Büntgen, U., P. J. Krusic, A. Piermattei, D. A. Coomes, J. Esper, V. S. Myglan, A. V. Kirdyanov, J. J. Camarero, A. Crivellaro, and C. Körner (2019). "Limited capacity of tree growth to mitigate the global greenhouse effect under predicted warming". Nature Communications 10.1, 1-6.

Burba, G. and D. Anderson (2007). Introduction to the Eddy Covariance Method: General Guidelines and Conventional Workflow. LI-COR Biosciences, 141 pp.

Campioli, M., S. Vicca, S. Luyssaert, J. Bilcke, E. Ceschia, F. S. Chapin III, P. Ciais, M. Fernández-Martínez, Y. Malhi, M. Obersteiner, D. Olefeldt, D. Papale, S. L. Piao, J. Peñuelas, P. F. Sullivan, X. Wang, T. Zenone, and I. A. Janssens (2015). "Biomass production efficiency controlled by management in temperate and boreal ecosystems". Nature Geoscience 8.11, 843-846. 
Camps-Valls, G., L. Martino, D. H. Svendsen, M. Campos-Taberner, J. Muñoz-Marí, V. Laparra, D. Luengo, and F. J. García-Haro (2018). "Physics-aware Gaussian processes in remote sensing". Applied Soft Computing 68, 69-82.

Canadell, J. G., C. Le Quere, M. R. Raupach, C. B. Field, E. T. Buitenhuis, P. Ciais, T. J. Conway, N. P. Gillett, R. A. Houghton, and G. Marland (2007). "Contributions to accelerating atmospheric $\mathrm{CO} 2$ growth from economic activity, carbon intensity, and efficiency of natural sinks". Proceedings of the National Academy of Sciences 104.47, 18866-18870.

Canadell, J. G., H. A. Mooney, D. D. Baldocchi, J. A. Berry, J. R. Ehleringer, C. B. Field, S. T. Gower, D. Y. Hollinger, J. E. Hunt, R. B. Jackson, S. W. Running, G. R. Shaver, W. Steffen, S. E. Trumbore, R. Valentini, and B. Y. Bond (2000). "Commentary: Carbon Metabolism of the Terrestrial Biosphere: A Multitechnique Approach for Improved Understanding”. Ecosystems $3.2,115-130$.

Carvalhais, N., M. Forkel, M. Khomik, J. Bellarby, M. Jung, M. Migliavacca, M. Mu, S. Saatchi, M. Santoro, M. Thurner, U. Weber, B. Ahrens, C. Beer, A. Cescatti, J. T. Randerson, and M. Reichstein (2014). "Global covariation of carbon turnover times with climate in terrestrial ecosystems”. Nature 514.7521, 213-217.

Carvalhais, N., M. Reichstein, P. Ciais, G. J. Collatz, M. D. Mahecha, L. Montagnani, D. Papale, S. Rambal, and J. Seixas (2010). "Identification of vegetation and soil carbon pools out of equilibrium in a process model via eddy covariance and biometric constraints". Global Change Biology 16.10, 2813-2829.

Chambers, J. Q., J. I. Fisher, H. Zeng, E. L. Chapman, D. B. Baker, and G. C. Hurtt (2007). "Hurricane Katrina's Carbon Footprint on U.S. Gulf Coast Forests". Science 318.5853, 11071107.

Chazdon, R. L., E. N. Broadbent, D. M. A. Rozendaal, F. Bongers, A. M. A. Zambrano, T. M. Aide, P. Balvanera, J. M. Becknell, V. Boukili, P. H. S. Brancalion, D. Craven, J. S. AlmeidaCortez, G. A. L. Cabral, B. d. Jong, J. S. Denslow, D. H. Dent, S. J. DeWalt, J. M. Dupuy, S. M. Durán, M. M. Espírito-Santo, M. C. Fandino, R. G. César, J. S. Hall, J. L. Hernández-Stefanoni, C. C. Jakovac, A. B. Junqueira, D. Kennard, S. G. Letcher, M. Lohbeck, M. Martínez-Ramos, P. Massoca, J. A. Meave, R. Mesquita, F. Mora, R. Muñoz, R. Muscarella, Y. R. F. Nunes, S. Ochoa-Gaona, E. Orihuela-Belmonte, M. Peña-Claros, E. A. Pérez-García, D. Piotto, J. S. Powers, J. Rodríguez-Velazquez, I. E. Romero-Pérez, J. Ruíz, J. G. Saldarriaga, A. SanchezAzofeifa, N. B. Schwartz, M. K. Steininger, N. G. Swenson, M. Uriarte, M. v. Breugel, H. v. d. Wal, M. D. M. Veloso, H. Vester, I. C. G. Vieira, T. V. Bentos, G. B. Williamson, and L. Poorter (2016). "Carbon sequestration potential of second-growth forest regeneration in the Latin American tropics". Science Advances 2.5, e1501639.

Chen, J. M., W. Ju, J. Cihlar, D. Price, J. Liu, W. Chen, J. Pan, A. Black, and A. Barr (2003). "Spatial distribution of carbon sources and sinks in Canada's forests". Tellus B 55.2, 622-641.

Chen, J., M. Falk, E. Euskirchen, K. T. P. U, T. H. Suchanek, S. L. Ustin, B. J. Bond, K. D. Brosofske, N. Phillips, and R. Bi (2002). "Biophysical controls of carbon flows in three 
successional Douglas-fir stands based on eddy-covariance measurements". Tree Physiology 22.2-3, 169-177.

Chu, H., D. D. Baldocchi, R. John, S. Wolf, and M. Reichstein (2017). "Fluxes all of the time? A primer on the temporal representativeness of FLUXNET". Journal of Geophysical Research: Biogeosciences 122.2, 289-307.

Ciais, P., A. J. Dolman, A. Bombelli, R. Duren, A. Peregon, P. J. Rayner, C. Miller, N. Gobron, G. Kinderman, G. Marland, N. Gruber, F. Chevallier, R. J. Andres, G. Balsamo, L. Bopp, F.-M. Bréon, G. Broquet, R. Dargaville, T. J. Battin, A. Borges, H. Bovensmann, M. Buchwitz, J. Butler, J. G. Canadell, R. B. Cook, R. DeFries, R. Engelen, K. R. Gurney, C. Heinze, M. Heimann, A. Held, M. Henry, B. Law, S. Luyssaert, J. Miller, T. Moriyama, C. Moulin, R. B. Myneni, C. Nussli, M. Obersteiner, D. Ojima, Y. Pan, J.-D. Paris, S. L. Piao, B. Poulter, S. Plummer, S. Quegan, P. Raymond, M. Reichstein, L. Rivier, C. Sabine, D. Schimel, O. Tarasova, R. Valentini, R. Wang, G. van der Werf, D. Wickland, M. Williams, and C. Zehner (2014a). "Current systematic carbon-cycle observations and the need for implementing a policy-relevant carbon observing system”. Biogeosciences 11.13, 3547-3602.

Ciais, P., M. Reichstein, N. Viovy, A. Granier, J. Ogée, V. Allard, M. Aubinet, N. Buchmann, C. Bernhofer, A. Carrara, F. Chevallier, N. De Noblet, A. D. Friend, P. Friedlingstein, T. Grünwald, B. Heinesch, P. Keronen, A. Knohl, G. Krinner, D. Loustau, G. Manca, G. Matteucci, F. Miglietta, J. M. Ourcival, D. Papale, K. Pilegaard, S. Rambal, G. Seufert, J. F. Soussana, M. J. Sanz, E. D. Schulze, T. Vesala, and R. Valentini (2005). "Europe-wide reduction in primary productivity caused by the heat and drought in 2003". Nature 437.7058, 529-533.

Ciais, P., S. Besnard, Y. Yao, J. Chave, M. Herold, S. Piao, B. Poulter, T. Pugh, M. Reichstein, D. M. Rozendaal, P. Stoy, C. Yue, and N. Carvalhais (2019). "The global forest carbon balance inferred from flux towers and forest age". In preparation.

Ciais, P., C. Sabine, G. Bala, L. Bopp, V. Brovkin, J. Canadell, A. Chhabra, R. DeFries, J. Galloway, M. Heimann, et al. (2014b). "Carbon and other biogeochemical cycles". In: Climate change 2013: the physical science basis. Contribution of Working Group I to the Fifth Assessment Report of the Intergovernmental Panel on Climate Change. Cambridge University Press, 465-570.

Clark, K. L., N. Skowronski, and J. Hom (2010). "Invasive insects impact forest carbon dynamics". Global Change Biology 16.1, 88-101.

Coursolle, C., H. A. Margolis, M. A. Giasson, P. Y. Bernier, B. D. Amiro, M. A. Arain, A. G. Barr, T. A. Black, M. L. Goulden, J. H. McCaughey, J. M. Chen, A. L. Dunn, R. F. Grant, and P. M. Lafleur (2012). "Influence of stand age on the magnitude and seasonality of carbon fluxes in Canadian forests". Agricultural and Forest Meteorology 165, 136-148.

Coursolle, C., H. A. Margolis, A. G. Barr, T. A. Black, B. D. Amiro, J. H. McCaughey, L. B. Flanagan, P. M. Lafleur, N. T. Roulet, C. P.-A. Bourque, M. A. Arain, S. C. Wofsy, A. Dunn, K. Morgenstern, A. L. Orchansky, P. Y. Bernier, J. M. Chen, J. Kidston, N. Saigusa, and 
N. Hedstrom (2006). "Late-summer carbon fluxes from Canadian forests and peatlands along an east-west continental transect". Canadian Journal of Forest Research 36.3, 783-800.

Curtis, P. S. and C. M. Gough (2018). "Forest aging, disturbance and the carbon cycle". New Phytologist 219.4, 1188-1193.

Dee, D. P., S. M. Uppala, A. J. Simmons, P. Berrisford, P. Poli, S. Kobayashi, U. Andrae, M. A. Balmaseda, G. Balsamo, P. Bauer, P. Bechtold, A. C. M. Beljaars, L. van de Berg, J. Bidlot, N. Bormann, C. Delsol, R. Dragani, M. Fuentes, A. J. Geer, L. Haimberger, S. B. Healy, H. Hersbach, E. V. Hólm, L. Isaksen, P. Kållberg, M. Köhler, M. Matricardi, A. P. McNally, B. M. Monge-Sanz, J.-J. Morcrette, B.-K. Park, C. Peubey, P. de Rosnay, C. Tavolato, J.-N. Thépaut, and F. Vitart (2011). "The ERA-Interim reanalysis: configuration and performance of the data assimilation system". Quarterly Journal of the Royal Meteorological Society 137.656, 553-597.

Desai, A. R. (2014). "Influence and predictive capacity of climate anomalies on daily to decadal extremes in canopy photosynthesis". Photosynthesis Research 119.1, 31-47.

DeVries, B., M. Decuyper, J. Verbesselt, A. Zeileis, M. Herold, and S. Joseph (2015). “Tracking disturbance-regrowth dynamics in tropical forests using structural change detection and Landsat time series". Remote Sensing of Environment 169, 320-334.

Didan, K. (2015). MOD13C1 MODIS/Terra Vegetation Indices 16-Day L3 Global 0.05Deg CMG V006. NASA EOSDIS Land Processes DAAC.

Donato, D. C., B. J. Harvey, W. H. Romme, M. Simard, and M. G. Turner (2013). "Bark beetle effects on fuel profiles across a range of stand structures in Douglas-fir forests of Greater Yellowstone". Ecological Applications 23.1, 3-20.

Entry, J. A. and W. H. Emmingham (1995). "Influence of forest age on nutrient availability and storage in coniferous soils of the Oregon Coast Range". Canadian Journal of Forest Research $25.1,114-120$.

Fang, J., Z. Guo, H. Hu, T. Kato, H. Muraoka, and Y. Son (2014). "Forest biomass carbon sinks in East Asia, with special reference to the relative contributions of forest expansion and forest growth”. Global Change Biology 20.6, 2019-2030.

FAO (2006). Global Forest Resources Assessment 2005: progress towards sustainable forest management. 147. Rome: Food and Agriculture Organization of the United Nations. 320 pp.

- (2010). Global forest resources assessment 2010: Main report. 163. Rome: Food and Agriculture Organization of the United Nations. $340 \mathrm{pp}$.

- (2015). Global forest resources assessment 2015. Food and Agriculture Organization of the United Nations. 253 pp.

Fernández-Martínez, M., S. Vicca, I. A. Janssens, J. Sardans, S. Luyssaert, M. Campioli, F. S. Chapin III, P. Ciais, Y. Malhi, M. Obersteiner, D. Papale, S. L. Piao, M. Reichstein, F. Rodà, and J. Peñuelas (2014). "Nutrient availability as the key regulator of global forest carbon balance". Nature Climate Change 4.6, 471-476. 
Fick, S. E. and R. J. Hijmans (2017). "WorldClim 2: new 1-km spatial resolution climate surfaces for global land areas". International Journal of Climatology 37.12, 4302-4315.

Field, C. B., J. T. Randerson, and C. M. Malmström (1995). "Global net primary production: Combining ecology and remote sensing”. Remote Sensing of Environment 51.1, 74-88.

Foken, T., M. Aubinet, and R. Leuning (2012). "The eddy covariance method". In: Eddy covariance. Springer, 1-19.

Frank, D., M. Reichstein, M. Bahn, K. Thonicke, D. Frank, M. D. Mahecha, P. Smith, M. v. d. Velde, S. Vicca, F. Babst, C. Beer, N. Buchmann, J. G. Canadell, P. Ciais, W. Cramer, A. Ibrom, F. Miglietta, B. Poulter, A. Rammig, S. I. Seneviratne, A. Walz, M. Wattenbach, M. A. Zavala, and J. Zscheischler (2015). "Effects of climate extremes on the terrestrial carbon cycle: concepts, processes and potential future impacts". Global Change Biology 21.8, 2861-2880.

Frankenberg, C., J. B. Fisher, J. Worden, G. Badgley, S. S. Saatchi, J.-E. Lee, G. C. Toon, A. Butz, M. Jung, A. Kuze, and T. Yokota (2011). "New global observations of the terrestrial carbon cycle from GOSAT: Patterns of plant fluorescence with gross primary productivity". Geophysical Research Letters 38.17.

Franklin, J. F., T. A. Spies, R. V. Pelt, A. B. Carey, D. A. Thornburgh, D. R. Berg, D. B. Lindenmayer, M. E. Harmon, W. S. Keeton, D. C. Shaw, K. Bible, and J. Chen (2002). "Disturbances and structural development of natural forest ecosystems with silvicultural implications, using Douglas-fir forests as an example”. Forest Ecology and Management 155.1, 399-423.

Friedl, M. A., D. Sulla-Menashe, B. Tan, A. Schneider, N. Ramankutty, A. Sibley, and X. Huang (2010). "MODIS Collection 5 global land cover: Algorithm refinements and characterization of new datasets". Remote Sensing of Environment 114.1, 168-182.

Friedlingstein, P., R. M. Andrew, J. Rogelj, G. P. Peters, J. G. Canadell, R. Knutti, G. Luderer, M. R. Raupach, M. Schaeffer, D. P. van Vuuren, and C. Le Quéré (2014). "Persistent growth of $\mathrm{CO}_{2}$ emissions and implications for reaching climate targets". Nature Geoscience 7.10, 709-715.

Friedlingstein, P. (2015). "Carbon cycle feedbacks and future climate change". Philosophical Transactions of the Royal Society A: Mathematical, Physical and Engineering Sciences 373.2054, 20140421.

Friedlingstein, P., M. Meinshausen, V. K. Arora, C. D. Jones, A. Anav, S. K. Liddicoat, and R. Knutti (2013). "Uncertainties in CMIP5 Climate Projections due to Carbon Cycle Feedbacks". Journal of Climate 27.2, 511-526.

Friend, A. D., W. Lucht, T. T. Rademacher, R. Keribin, R. Betts, P. Cadule, P. Ciais, D. B. Clark, R. Dankers, P. D. Falloon, A. Ito, R. Kahana, A. Kleidon, M. R. Lomas, K. Nishina, S. Ostberg, R. Pavlick, P. Peylin, S. Schaphoff, N. Vuichard, L. Warszawski, A. Wiltshire, and F. I. Woodward (2014). "Carbon residence time dominates uncertainty in terrestrial vegetation responses to future climate and atmospheric $\mathrm{CO}_{2}$ ". Proceedings of the National Academy of Sciences 111.9, 3280-3285. 
Gao, S., T. Zhou, X. Zhao, D. Wu, Z. Li, H. Wu, L. Du, and H. Luo (2016). "Age and climate contribution to observed forest carbon sinks in East Asia". Environmental Research Letters 11.3, 034021.

Giglio, L., L. Boschetti, D. P. Roy, M. L. Humber, and C. O. Justice (2018). "The Collection 6 MODIS burned area mapping algorithm and product". Remote Sensing of Environment 217, $72-85$.

Giorgi, F. (2006). "Climate change hot-spots”. Geophysical Research Letters 33.8.

Gorelick, N., M. Hancher, M. Dixon, S. Ilyushchenko, D. Thau, and R. Moore (2017). "Google Earth Engine: Planetary-scale geospatial analysis for everyone". Remote Sensing of Environment 202, 18-27.

Gough, C. M., P. S. Curtis, B. S. Hardiman, C. M. Scheuermann, and B. Bond-Lamberty (2016). "Disturbance, complexity, and succession of net ecosystem production in North America's temperate deciduous forests". Ecosphere 7.6, e01375.

Gough, C. M., C. S. Vogel, K. H. Harrold, K. George, and P. S. Curtis (2007). "The legacy of harvest and fire on ecosystem carbon storage in a north temperate forest". Global Change Biology 13.9, 1935-1949.

Gough, C. M., C. S. Vogel, H. P. Schmid, and P. S. Curtis (2008). "Controls on Annual Forest Carbon Storage: Lessons from the Past and Predictions for the Future". BioScience 58.7, 609-622.

Gower, S. T. (2003). "Patterns and Mechanisms of the Forest Carbon Cycle". Annual Review of Environment and Resources 1, 169-204.

Gray, A. N., T. R. Whittier, and M. E. Harmon (2016). "Carbon stocks and accumulation rates in Pacific Northwest forests: role of stand age, plant community, and productivity". Ecosphere 7.1, e01224.

Guanter, L., Y. Zhang, M. Jung, J. Joiner, M. Voigt, J. A. Berry, C. Frankenberg, A. R. Huete, P. Zarco-Tejada, J.-E. Lee, M. S. Moran, G. Ponce-Campos, C. Beer, G. Camps-Valls, N. Buchmann, D. Gianelle, K. Klumpp, A. Cescatti, J. M. Baker, and T. J. Griffis (2014). "Global and time-resolved monitoring of crop photosynthesis with chlorophyll fluorescence". Proceedings of the National Academy of Sciences 111.14, E1327-E1333.

Hansen, M. C., P. V. Potapov, R. Moore, M. Hancher, S. A. Turubanova, A. Tyukavina, D. Thau, S. V. Stehman, S. J. Goetz, T. R. Loveland, A. Kommareddy, A. Egorov, L. Chini, C. O. Justice, and J. R. G. Townshend (2013). "High-Resolution Global Maps of 21st-Century Forest Cover Change". Science 342.6160, 850-853.

Harmon, M. E., B. Bond-Lamberty, J. Tang, and R. Vargas (2011). "Heterotrophic respiration in disturbed forests: A review with examples from North America". Journal of Geophysical Research: Biogeosciences 116.G4.

Harmon, M. E., W. K. Ferrell, and J. F. Franklin (1990). "Effects on Carbon Storage of Conversion of Old-Growth Forests to Young Forests". Science 247.4943, 699-702. 
Harris, I., P. Jones, T. Osborn, and D. Lister (2014). "Updated high-resolution grids of monthly climatic observations - the CRU TS3.10 Dataset". International Journal of Climatology 34, 623-642.

Hengl, T., J. M. d. Jesus, R. A. MacMillan, N. H. Batjes, G. B. M. Heuvelink, E. Ribeiro, A. Samuel-Rosa, B. Kempen, J. G. B. Leenaars, M. G. Walsh, and M. R. Gonzalez (2014). "SoilGrids1km — Global Soil Information Based on Automated Mapping". PLOS ONE 9.8, e105992.

Hicke, J. A., C. D. Allen, A. R. Desai, M. C. Dietze, R. J. Hall, E. H. (Ted) Hogg, D. M. Kashian, D. Moore, K. F. Raffa, R. N. Sturrock, and J. Vogelmann (2012). "Effects of biotic disturbances on forest carbon cycling in the United States and Canada". Global Change Biology 18.1, 7-34.

Hinton, G., L. Deng, D. Yu, G. E. Dahl, A. Mohamed, N. Jaitly, A. Senior, V. Vanhoucke, P. Nguyen, T. N. Sainath, and B. Kingsbury (2012). "Deep Neural Networks for Acoustic Modeling in Speech Recognition: The Shared Views of Four Research Groups". IEEE Signal Processing Magazine 29.6, 82-97.

Hochreiter, S. and J. Schmidhuber (1997). "Long Short-Term Memory”. Neural Comput. 9.8, 1735-1780.

Hoerling, M., J. Eischeid, J. Perlwitz, X. Quan, T. Zhang, and P. Pegion (2011). “On the Increased Frequency of Mediterranean Drought”. Journal of Climate 25.6, 2146-2161.

Houghton, R. A. and A. A. Nassikas (2017). "Global and regional fluxes of carbon from land use and land cover change 1850-2015”. Global Biogeochemical Cycles 31.3, 456-472.

Huang, C., S. N. Goward, J. G. Masek, N. Thomas, Z. Zhu, and J. E. Vogelmann (2010). “An automated approach for reconstructing recent forest disturbance history using dense Landsat time series stacks". Remote Sensing of Environment 114.1, 183-198.

Huete, A., K. Didan, T. Miura, E. P. Rodriguez, X. Gao, and L. G. Ferreira (2002). "Overview of the radiometric and biophysical performance of the MODIS vegetation indices". Remote Sensing of Environment 83.1, 195-213.

Irvine, J., B. E. Law, and M. R. Kurpius (2005). "Coupling of canopy gas exchange with root and rhizosphere respiration in a semi-arid forest". Biogeochemistry 73.1, 271-282.

Ito, A. (2011). "A historical meta-analysis of global terrestrial net primary productivity: are estimates converging?" Global Change Biology 17.10, 3161-3175.

Janssens, I. A., W. Dieleman, S. Luyssaert, J.-A. Subke, M. Reichstein, R. Ceulemans, P. Ciais, A. J. Dolman, J. Grace, G. Matteucci, D. Papale, S. L. Piao, E.-D. Schulze, J. Tang, and B. E. Law (2010). "Reduction of forest soil respiration in response to nitrogen deposition". Nature Geoscience 3.5, 315-322.

Johnson, D. W. and P. S. Curtis (2001). "Effects of forest management on soil C and N storage: meta analysis". Forest Ecology and Management 140.2, 227-238.

Johnson, M. O., D. Galbraith, M. Gloor, H. D. Deurwaerder, M. Guimberteau, A. Rammig, K. Thonicke, H. Verbeeck, C. v. Randow, A. Monteagudo, O. L. Phillips, R. J. W. Brienen, 
T. R. Feldpausch, G. L. Gonzalez, S. Fauset, C. A. Quesada, B. Christoffersen, P. Ciais, G. Sampaio, B. Kruijt, P. Meir, P. Moorcroft, K. Zhang, E. Alvarez-Davila, A. A. d. Oliveira, I. Amaral, A. Andrade, L. E. O. C. Aragao, A. Araujo-Murakami, E. J. M. M. Arets, L. Arroyo, G. A. Aymard, C. Baraloto, J. Barroso, D. Bonal, R. Boot, J. Camargo, J. Chave, A. Cogollo, F. C. Valverde, A. C. L. d. Costa, A. D. Fiore, L. Ferreira, N. Higuchi, E. N. Honorio, T. J. Killeen, S. G. Laurance, W. F. Laurance, J. Licona, T. Lovejoy, Y. Malhi, B. Marimon, B. H. Marimon, D. C. L. Matos, C. Mendoza, D. A. Neill, G. Pardo, M. Peña-Claros, N. C. A. Pitman, L. Poorter, A. Prieto, H. Ramirez-Angulo, A. Roopsind, A. Rudas, R. P. Salomao, M. Silveira, J. Stropp, H. t. Steege, J. Terborgh, R. Thomas, M. Toledo, A. Torres-Lezama, G. M. F. v. d. Heijden, R. Vasquez, I. C. G. Vieira, E. Vilanova, V. A. Vos, and T. R. Baker (2016). "Variation in stem mortality rates determines patterns of above-ground biomass in Amazonian forests: implications for dynamic global vegetation models". Global Change Biology 22.12, 3996-4013.

Joiner, J., L. Guanter, R. Lindstrot, M. Voigt, A. P. Vasilkov, E. M. Middleton, K. F. Huemmrich, Y. Yoshida, and C. Frankenberg (2013). "Global monitoring of terrestrial chlorophyll fluorescence from moderate-spectral-resolution near-infrared satellite measurements: methodology, simulations, and application to GOME-2". Atmospheric Measurement Techniques 6.10, 28032823.

Joiner, J., Y. Yoshida, A. P. Vasilkov, K. Schaefer, M. Jung, L. Guanter, Y. Zhang, S. Garrity, E. M. Middleton, K. F. Huemmrich, L. Gu, and L. Belelli Marchesini (2014). "The seasonal cycle of satellite chlorophyll fluorescence observations and its relationship to vegetation phenology and ecosystem atmosphere carbon exchange". Remote Sensing of Environment 152, 375-391.

Jung, M., M. Reichstein, H. A. Margolis, A. Cescatti, A. D. Richardson, M. A. Arain, A. Arneth, C. Bernhofer, D. Bonal, J. Chen, D. Gianelle, N. Gobron, G. Kiely, W. Kutsch, G. Lasslop, B. E. Law, A. Lindroth, L. Merbold, L. Montagnani, E. J. Moors, D. Papale, M. Sottocornola, F. Vaccari, and C. Williams (2011). "Global patterns of land-atmosphere fluxes of carbon dioxide, latent heat, and sensible heat derived from eddy covariance, satellite, and meteorological observations". Journal of Geophysical Research: Biogeosciences 116.G3.

Jung, M. and J. Zscheischler (2013). "A Guided Hybrid Genetic Algorithm for Feature Selection with Expensive Cost Functions". Procedia Computer Science 18, 2337-2346.

Karpatne, A., G. Atluri, J. Faghmous, M. Steinbach, A. Banerjee, A. Ganguly, S. Shekhar, N. Samatova, and V. Kumar (2017a). "Theory-guided Data Science: A New Paradigm for Scientific Discovery from Data”. IEEE Transactions on Knowledge and Data Engineering 29.10, 2318-2331. arXiv: 1612 .08544.

Karpatne, A., W. Watkins, J. Read, and V. Kumar (2017b). "Physics-guided Neural Networks (PGNN): An Application in Lake Temperature Modeling”. arXiv:1710.11431 [physics, stat]. arXiv: 1710.11431. 
Kashian, D. M., W. H. Romme, D. B. Tinker, M. G. Turner, and M. G. Ryan (2013). "Postfire changes in forest carbon storage over a 300-year chronosequence of Pinus contorta-dominated forests". Ecological Monographs 83.1, 49-66.

Kasting, J. F. (1989). "Long-term stability of the earth's climate”. Palaeogeography, Palaeoclimatology, Palaeoecology 75.1, 83-95.

Katul, G. G., S. Palmroth, and R. Oren (2009). "Leaf stomatal responses to vapour pressure deficit under current and $\mathrm{CO}_{2}$-enriched atmosphere explained by the economics of gas exchange". Plant, Cell \& Environment 32.8, 968-979.

Katul, G., S. Manzoni, S. Palmroth, and R. Oren (2010). "A stomatal optimization theory to describe the effects of atmospheric $\mathrm{CO}_{2}$ on leaf photosynthesis and transpiration". Annals of Botany 105.3, 431-442.

Kennedy, R. E., Z. Yang, and W. B. Cohen (2010). "Detecting trends in forest disturbance and recovery using yearly Landsat time series: 1. LandTrendr - Temporal segmentation algorithms". Remote Sensing of Environment 114.12, 2897-2910.

Kim, J.-S., J.-S. Kug, J.-H. Yoon, and S.-J. Jeong (2016). "Increased Atmospheric CO2 Growth Rate during El Niño Driven by Reduced Terrestrial Productivity in the CMIP5 ESMs". Journal of Climate 29.24, 8783-8805.

Kingma, D. P. and J. Ba (2014). "Adam: A Method for Stochastic Optimization". arXiv:1412.6980 [cs].

Köhler, P., L. Guanter, and J. Joiner (2015). "A linear method for the retrieval of sun-induced chlorophyll fluorescence from GOME-2 and SCIAMACHY data". Atmospheric Measurement Techniques 8.6, 2589-2608.

Köhler, P., C. Frankenberg, T. S. Magney, L. Guanter, J. Joiner, and J. Landgraf (2018). "Global Retrievals of Solar-Induced Chlorophyll Fluorescence With TROPOMI: First Results and Intersensor Comparison to OCO-2". Geophysical Research Letters 45.19, 10, 456-10, 463.

Kosugi, Y., S. Takanashi, M. Tani, S. Ohkubo, N. Matsuo, M. Itoh, S. Noguchi, and A. R. Nik (2012). "Effect of inter-annual climate variability on evapotranspiration and canopy CO2 exchange of a tropical rainforest in Peninsular Malaysia". Journal of Forest Research 17.3, 227-240.

Kuhn, M. (2008). "Building Predictive Models in R Using the caret Package". Journal of Statistical Software 28.1, 1-26.

Kumar, J., F. M. Hoffman, W. W. Hargrove, and N. Collier (2016). "Understanding the representativeness of FLUXNET for upscaling carbon flux from eddy covariance measurements". Earth System Science Data Discussions, 1-25.

Kursa, M. B. and W. R. Rudnicki (2010). "Feature Selection with the Boruta Package". Journal of Statistical Software 36.1, 1-13. 
Kurz, W. A., C. C. Dymond, G. Stinson, G. J. Rampley, E. T. Neilson, A. L. Carroll, T. Ebata, and L. Safranyik (2008). "Mountain pine beetle and forest carbon feedback to climate change". Nature 452.7190, 987-990.

Kutsch, W. L. and P. Kolari (2015). "Data quality and the role of nutrients in forest carbon-use efficiency". Nature Climate Change 5.11, 959-960.

Larcher, W. (2003). Physiological Plant Ecology: Ecophysiology and Stress Physiology of Functional Groups. 4th ed. Berlin Heidelberg: Springer-Verlag.

Lasslop, G., M. Reichstein, D. Papale, A. D. Richardson, A. Arneth, A. Barr, P. Stoy, and G. Wohlfahrt (2010). "Separation of net ecosystem exchange into assimilation and respiration using a light response curve approach: critical issues and global evaluation”. Global Change Biology 16.1, 187-208.

Lauerwald, R., G. G. Laruelle, J. Hartmann, P. Ciais, and P. A. G. Regnier (2015). "Spatial patterns in CO2 evasion from the global river network". Global Biogeochemical Cycles 29.5, 534-554.

Law, B. E., O. J. Sun, J. Campbell, S. V. Tuyl, and P. E. Thornton (2003). "Changes in carbon storage and fluxes in a chronosequence of ponderosa pine". Global Change Biology 9.4, 510-524.

Law, B. E., E. Falge, L. Gu, D. D. Baldocchi, P. Bakwin, P. Berbigier, K. Davis, A. J. Dolman, M. Falk, J. D. Fuentes, A. Goldstein, A. Granier, A. Grelle, D. Hollinger, I. A. Janssens, P. Jarvis, N. O. Jensen, G. Katul, Y. Mahli, G. Matteucci, T. Meyers, R. Monson, W. Munger, W. Oechel, R. Olson, K. Pilegaard, K. T. Paw U, H. Thorgeirsson, R. Valentini, S. Verma, T. Vesala, K. Wilson, and S. Wofsy (2002). "Environmental controls over carbon dioxide and water vapor exchange of terrestrial vegetation". Agricultural and Forest Meteorology 113.1, 97-120.

Law, B. E., T. W. Hudiburg, L. T. Berner, J. J. Kent, P. C. Buotte, and M. E. Harmon (2018). "Land use strategies to mitigate climate change in carbon dense temperate forests". Proceedings of the National Academy of Sciences 115.14, 3663-3668.

Le Quéré, C., R. Moriarty, R. M. Andrew, J. G. Canadell, S. Sitch, J. I. Korsbakken, P. Friedlingstein, G. P. Peters, R. J. Andres, T. A. Boden, R. A. Houghton, J. I. House, R. F. Keeling, P. Tans, A. Arneth, D. C. E. Bakker, L. Barbero, L. Bopp, J. Chang, F. Chevallier, L. P. Chini, P. Ciais, M. Fader, R. A. Feely, T. Gkritzalis, I. Harris, J. Hauck, T. Ilyina, A. K. Jain, E. Kato, V. Kitidis, K. Klein Goldewijk, C. Koven, P. Landschützer, S. K. Lauvset, N. Lefèvre, A. Lenton, I. D. Lima, N. Metzl, F. Millero, D. R. Munro, A. Murata, J. E. M. S. Nabel, S. Nakaoka, Y. Nojiri, K. O \\&amp;apos;Brien, A. Olsen, T. Ono, F. F. Pérez, B. Pfeil, D. Pierrot, B. Poulter, G. Rehder, C. Rödenbeck, S. Saito, U. Schuster, J. Schwinger, R. Séférian, T. Steinhoff, B. D. Stocker, A. J. Sutton, T. Takahashi, B. Tilbrook, I. T. van der Laan-Luijkx, G. R. van der Werf, S. van Heuven, D. Vandemark, N. Viovy, A. Wiltshire, S. Zaehle, and N. Zeng (2015). “Global Carbon Budget 2015”. Earth System Science Data 7.2, 349-396. 
Le Quéré, C., R. M. Andrew, P. Friedlingstein, S. Sitch, J. Pongratz, A. C. Manning, J. I. Korsbakken, G. P. Peters, J. G. Canadell, R. B. Jackson, T. A. Boden, P. P. Tans, O. D. Andrews, V. K. Arora, D. C. E. Bakker, L. Barbero, M. Becker, R. A. Betts, L. Bopp, F. Chevallier, L. P. Chini, P. Ciais, C. E. Cosca, J. Cross, K. Currie, T. Gasser, I. Harris, J. Hauck, V. Haverd, R. A. Houghton, C. W. Hunt, G. Hurtt, T. Ilyina, A. K. Jain, E. Kato, M. Kautz, R. F. Keeling, K. Klein Goldewijk, A. Körtzinger, P. Landschützer, N. Lefèvre, A. Lenton, S. Lienert, I. Lima, D. Lombardozzi, N. Metzl, F. Millero, P. M. S. Monteiro, D. R. Munro, J. E. M. S. Nabel, S.-i. Nakaoka, Y. Nojiri, X. A. Padin, A. Peregon, B. Pfeil, D. Pierrot, B. Poulter, G. Rehder, J. Reimer, C. Rödenbeck, J. Schwinger, R. Séférian, I. Skjelvan, B. D. Stocker, H. Tian, B. Tilbrook, F. N. Tubiello, I. T. van der Laan-Luijkx, G. R. van der Werf, S. van Heuven, N. Viovy, N. Vuichard, A. P. Walker, A. J. Watson, A. J. Wiltshire, S. Zaehle, and D. Zhu (2018). "Global Carbon Budget 2017”. Earth System Science Data 10.1, 405-448.

Le Quéré, C., M. R. Raupach, J. G. Canadell, G. Marland, C. Le Quéré, M. R. Raupach, J. G. Canadell, G. Marland, L. Bopp, P. Ciais, T. J. Conway, S. C. Doney, R. A. Feely, P. Foster, P. Friedlingstein, K. Gurney, R. A. Houghton, J. I. House, C. Huntingford, P. E. Levy, M. R. Lomas, J. Majkut, N. Metzl, J. P. Ometto, G. P. Peters, I. C. Prentice, J. T. Randerson, S. W. Running, J. L. Sarmiento, U. Schuster, S. Sitch, T. Takahashi, N. Viovy, G. R. van der Werf, and F. I. Woodward (2009). "Trends in the sources and sinks of carbon dioxide". Nature Geoscience 2.12, 831-836.

LeCun, Y., Y. Bengio, and G. Hinton (2015). “Deep learning”. Nature 521.7553, 436-444.

Lee, J.-E., C. Frankenberg, C. van der Tol, J. A. Berry, L. Guanter, C. K. Boyce, J. B. Fisher, E. Morrow, J. R. Worden, S. Asefi, G. Badgley, and S. Saatchi (2013). "Forest productivity and water stress in Amazonia: observations from GOSAT chlorophyll fluorescence". Proceedings of the Royal Society B: Biological Sciences 280.1761, 20130171.

Lewis, S. L., B. Sonké, T. Sunderland, S. K. Begne, G. Lopez-Gonzalez, G. M. F. van der Heijden, O. L. Phillips, K. Affum-Baffoe, T. R. Baker, L. Banin, J.-F. Bastin, H. Beeckman, P. Boeckx, J. Bogaert, C. De Cannière, E. Chezeaux, C. J. Clark, M. Collins, G. Djagbletey, M. N. K. Djuikouo, V. Droissart, J.-L. Doucet, C. E. N. Ewango, S. Fauset, T. R. Feldpausch, E. G. Foli, J.-F. Gillet, A. C. Hamilton, D. J. Harris, T. B. Hart, T. de Haulleville, A. Hladik, K. Hufkens, D. Huygens, P. Jeanmart, K. J. Jeffery, E. Kearsley, M. E. Leal, J. Lloyd, J. C. Lovett, J.-R. Makana, Y. Malhi, A. R. Marshall, L. Ojo, K. S.-H. Peh, G. Pickavance, J. R. Poulsen, J. M. Reitsma, D. Sheil, M. Simo, K. Steppe, H. E. Taedoumg, J. Talbot, J. R. D. Taplin, D. Taylor, S. C. Thomas, B. Toirambe, H. Verbeeck, J. Vleminckx, L. J. T. White, S. Willcock, H. Woell, and L. Zemagho (2013). "Above-ground biomass and structure of 260 African tropical forests". Philosophical Transactions of the Royal Society B: Biological Sciences 368.1625, 20120295.

Li, W., N. MacBean, P. Ciais, P. Defourny, C. Lamarche, S. Bontemps, R. A. Houghton, and S. Peng (2018). "Gross and net land cover changes in the main plant functional types derived from the annual ESA CCI land cover maps (1992-2015)". Earth System Science Data 10.1, 219-234. 
Lindauer, M., H. P. Schmid, R. Grote, M. Mauder, R. Steinbrecher, and B. Wolpert (2014). "Net ecosystem exchange over a non-cleared wind-throw-disturbed upland spruce forest-Measurements and simulations". Agricultural and Forest Meteorology 197, 219-234.

Lindroth, A., F. Lagergren, M. Aurela, B. Bjarnadottir, T. Christensen, E. Dellwik, A. Grelle, A. Ibrom, T. Johansson, H. Lankreijer, S. Launiainen, T. Laurila, M. Mölder, E. Nikinmaa, K. Pilegaard, B. D. Sigurdsson, and T. Vesala (2008). "Leaf area index is the principal scaling parameter for both gross photosynthesis and ecosystem respiration of Northern deciduous and coniferous forests". Tellus B 60.2, 129-142.

Liu, L., Y. Zhang, S. Wu, S. Li, and D. Qin (2018). "Water memory effects and their impacts on global vegetation productivity and resilience”. Scientific Reports 8.1, 2962.

Liu, S., B. Bond-Lamberty, J. A. Hicke, R. Vargas, S. Zhao, J. Chen, S. L. Edburg, Y. Hu, J. Liu, A. D. McGuire, J. Xiao, R. Keane, W. Yuan, J. Tang, Y. Luo, C. Potter, and J. Oeding (2011). "Simulating the impacts of disturbances on forest carbon cycling in North America: Processes, data, models, and challenges". Journal of Geophysical Research: Biogeosciences 116.G4.

Liu, Y., C. R. Schwalm, K. E. Samuels-Crow, and K. Ogle (2019). "Ecological memory of daily carbon exchange across the globe and its importance in drylands". Ecology Letters 0.0.

Lobell, D. B., A. Sibley, and J. Ivan Ortiz-Monasterio (2012). "Extreme heat effects on wheat senescence in India". Nature Climate Change 2.3, 186-189.

Loboda, T. V. and D. Chen (2017). "Spatial distribution of young forests and carbon fluxes within recent disturbances in Russia". Global Change Biology 23.1, 138-153.

Lovett, G. M., J. J. Cole, and M. L. Pace (2006). "Is Net Ecosystem Production Equal to Ecosystem Carbon Accumulation?” Ecosystems 9.1, 152-155.

Luyssaert, S., E.-D. Schulze, A. Börner, A. Knohl, D. Hessenmöller, B. E. Law, P. Ciais, and J. Grace (2008). “Old-growth forests as global carbon sinks”. Nature 455.7210, 213-215.

Marino, G. P., D. P. Kaiser, L. Gu, and D. M. Ricciuto (2011). "Reconstruction of false spring occurrences over the southeastern United States, 1901-2007: an increasing risk of spring freeze damage?" Environmental Research Letters 6.2, 024015.

Meigs, G. W., D. C. Donato, J. L. Campbell, J. G. Martin, and B. E. Law (2009). "Forest Fire Impacts on Carbon Uptake, Storage, and Emission: The Role of Burn Severity in the Eastern Cascades, Oregon". Ecosystems 12.8, 1246-1267.

Menzel, A., T. H. Sparks, N. Estrella, E. Koch, A. Aasa, R. Ahas, K. Alm-Kübler, P. Bissolli, O. Braslavská, A. Briede, F. M. Chmielewski, Z. Crepinsek, Y. Curnel, Å. Dahl, C. Defila, A. Donnelly, Y. Filella, K. Jatczak, F. Måge, A. Mestre, Ø. Nordli, J. Peñuelas, P. Pirinen, V. Remišová, H. Scheifinger, M. Striz, A. Susnik, A. J. H. V. Vliet, F.-E. Wielgolaski, S. Zach, and A. Zust (2006). "European phenological response to climate change matches the warming pattern". Global Change Biology 12.10, 1969-1976.

Mitchard, E. T. A., T. R. Feldpausch, R. J. W. Brienen, G. Lopez-Gonzalez, A. Monteagudo, T. R. Baker, S. L. Lewis, J. Lloyd, C. A. Quesada, M. Gloor, H. t. Steege, P. Meir, E. Alvarez, A. 
Araujo-Murakami, L. E. O. C. Aragão, L. Arroyo, G. Aymard, O. Banki, D. Bonal, S. Brown, F. I. Brown, C. E. Cerón, V. C. Moscoso, J. Chave, J. A. Comiskey, F. Cornejo, M. C. Medina, L. D. Costa, F. R. C. Costa, A. D. Fiore, T. F. Domingues, T. L. Erwin, T. Frederickson, N. Higuchi, E. N. H. Coronado, T. J. Killeen, W. F. Laurance, C. Levis, W. E. Magnusson, B. S. Marimon, B. H. M. Junior, I. M. Polo, P. Mishra, M. T. Nascimento, D. Neill, M. P. N. Vargas, W. A. Palacios, A. Parada, G. P. Molina, M. Peña-Claros, N. Pitman, C. A. Peres, L. Poorter, A. Prieto, H. Ramirez-Angulo, Z. R. Correa, A. Roopsind, K. H. Roucoux, A. Rudas, R. P. Salomão, J. Schietti, M. Silveira, P. F. d. Souza, M. K. Steininger, J. Stropp, J. Terborgh, R. Thomas, M. Toledo, A. Torres-Lezama, T. R. v. Andel, G. M. F. v. d. Heijden, I. C. G. Vieira, S. Vieira, E. Vilanova-Torre, V. A. Vos, O. Wang, C. E. Zartman, Y. Malhi, and O. L. Phillips (2014). "Markedly divergent estimates of Amazon forest carbon density from ground plots and satellites". Global Ecology and Biogeography 23.8, 935-946.

Molen, M. K. van der, A. J. Dolman, P. Ciais, T. Eglin, N. Gobron, B. E. Law, P. Meir, W. Peters, O. L. Phillips, M. Reichstein, T. Chen, S. C. Dekker, M. Doubková, M. A. Friedl, M. Jung, B. J. J. M. van den Hurk, R. A. M. de Jeu, B. Kruijt, T. Ohta, K. T. Rebel, S. Plummer, S. I. Seneviratne, S. Sitch, A. J. Teuling, G. R. van der Werf, and G. Wang (2011). "Drought and ecosystem carbon cycling”. Agricultural and Forest Meteorology 151.7, 765-773.

Monger, C., O. E. Sala, M. C. Duniway, H. Goldfus, I. A. Meir, R. M. Poch, H. L. Throop, and E. R. Vivoni (2015). "Legacy effects in linked ecological-soil-geomorphic systems of drylands". Frontiers in Ecology and the Environment 13.1, 13-19.

Montagnani, L., D. Zanotelli, M. Tagliavini, and E. Tomelleri (2018). "Timescale effects on the environmental control of carbon and water fluxes of an apple orchard". Ecology and Evolution $8.1,416-434$.

Monteith, J. and M. Unsworth (2007). Principles of environmental physics. Academic Press.

Montgomery, R. B. (1948). "Vertical eddy flux of heat in the atmosphere". Journal of Meteorology 5.6, 265-274.

Moore, D. J. P., N. A. Trahan, P. Wilkes, T. Quaife, B. B. Stephens, K. Elder, A. R. Desai, J. Negron, and R. K. Monson (2013). "Persistent reduced ecosystem respiration after insect disturbance in high elevation forests". Ecology Letters 16.6, 731-737.

Moreno, A., M. Neumann, and H. Hasenauer (2017). "Forest structures across Europe". Geoscience Data Journal 4.1, 17-28.

Musavi, T., M. Migliavacca, M. Reichstein, J. Kattge, C. Wirth, T. A. Black, I. Janssens, A. Knohl, D. Loustau, O. Roupsard, A. Varlagin, S. Rambal, A. Cescatti, D. Gianelle, H. Kondo, R. Tamrakar, and M. D. Mahecha (2017). "Stand age and species richness dampen interannual variation of ecosystem-level photosynthetic capacity". Nature Ecology \& Evolution 1.2, 0048.

Myneni, R. B., C. D. Keeling, C. J. Tucker, G. Asrar, and R. R. Nemani (1997). "Increased plant growth in the northern high latitudes from 1981 to 1991”. Nature 386.6626, 698-702. 
N'Guessan, A. E., J. K. N'dja, O. N. Yao, B. H. K. Amani, R. G. Z. Gouli, C. Piponiot, I. C. Zo-Bi, and B. Hérault (2019). "Drivers of biomass recovery in a secondary forested landscape of West Africa". Forest Ecology and Management 433, 325-331.

Nash, J. E. and J. V. Sutcliffe (1970). "River flow forecasting through conceptual models part I - A discussion of principles". Journal of Hydrology 10.3, 282-290.

Naudts, K., Y. Chen, M. J. McGrath, J. Ryder, A. Valade, J. Otto, and S. Luyssaert (2016). "Europe's forest management did not mitigate climate warming". Science 351.6273, 597-600.

Niu, S., Y. Luo, D. Li, S. Cao, J. Xia, J. Li, and M. D. Smith (2014). "Plant growth and mortality under climatic extremes: An overview". Environmental and Experimental Botany 98, 13-19.

Noormets, A., D. Epron, J. C. Domec, S. G. McNulty, T. Fox, G. Sun, and J. S. King (2015). "Effects of forest management on productivity and carbon sequestration: A review and hypothesis". Forest Ecology and Management 355, 124-140.

Noormets, A., S. G. McNulty, J.-C. Domec, M. Gavazzi, G. Sun, and J. S. King (2012). "The role of harvest residue in rotation cycle carbon balance in loblolly pine plantations. Respiration partitioning approach". Global Change Biology 18.10, 3186-3201.

Odum, E. P. (1969). "The Strategy of Ecosystem Development”. Science 164.3877, 262-270.

Ogle, K., J. J. Barber, G. A. Barron-Gafford, L. P. Bentley, J. M. Young, T. E. Huxman, M. E. Loik, and D. T. Tissue (2015). "Quantifying ecological memory in plant and ecosystem processes". Ecology Letters 18.3, 221-235.

Omlin, M. and P. Reichert (1999). "A comparison of techniques for the estimation of model prediction uncertainty”. Ecological Modelling 115.1, 45-59.

Oren, R., D. S. Ellsworth, K. H. Johnsen, N. Phillips, B. E. Ewers, C. Maier, K. V. R. Schäfer, H. McCarthy, G. Hendrey, S. G. McNulty, and G. G. Katul (2001). "Soil fertility limits carbon sequestration by forest ecosystems in a $\mathrm{CO}_{2}$-enriched atmosphere". Nature 411.6836, 469-472.

Pan, Y., J. M. Chen, R. Birdsey, K. McCullough, L. He, and F. Deng (2011a). "Age structure and disturbance legacy of North American forests". Biogeosciences 8.3, 715-732.

Pan, Y., R. A. Birdsey, J. Fang, R. Houghton, P. E. Kauppi, W. A. Kurz, O. L. Phillips, A. Shvidenko, S. L. Lewis, J. G. Canadell, P. Ciais, R. B. Jackson, S. W. Pacala, A. D. McGuire, S. Piao, A. Rautiainen, S. Sitch, and D. Hayes (2011b). "A Large and Persistent Carbon Sink in the World's Forests". Science 333.6045, 988-993.

Papale, D., M. Reichstein, M. Aubinet, E. Canfora, C. Bernhofer, W. Kutsch, B. Longdoz, S. Rambal, R. Valentini, T. Vesala, and D. Yakir (2006). "Towards a standardized processing of Net Ecosystem Exchange measured with eddy covariance technique: algorithms and uncertainty estimation". Biogeosciences 3.4, 571-583.

Parazoo, N. C., K. Bowman, J. B. Fisher, C. Frankenberg, D. B. A. Jones, A. Cescatti, O. Pérez-Priego, G. Wohlfahrt, and L. Montagnani (2014). "Terrestrial gross primary production 
inferred from satellite fluorescence and vegetation models". Global Change Biology 20.10, 3103-3121.

Paul-Limoges, E., T. A. Black, A. Christen, Z. Nesic, and R. S. Jassal (2015). "Effect of clearcut harvesting on the carbon balance of a Douglas-fir forest". Agricultural and Forest Meteorology 203, 30-42.

Peterson, G. D. (2002). “Contagious Disturbance, Ecological Memory, and the Emergence of Landscape Pattern”. Ecosystems 5.4, 329-338.

Peylin, P., R. M. Law, K. R. Gurney, F. Chevallier, A. R. Jacobson, T. Maki, Y. Niwa, P. K. Patra, W. Peters, P. J. Rayner, C. Rödenbeck, I. T. v. d. Laan-Luijkx, and X. Zhang (2013). "Global atmospheric carbon budget: results from an ensemble of atmospheric $\mathrm{CO}_{2}$ inversions". Biogeosciences 10.10, 6699-6720.

Piao, S., P. Ciais, P. Friedlingstein, P. Peylin, M. Reichstein, S. Luyssaert, H. Margolis, J. Fang, A. Barr, A. Chen, A. Grelle, D. Y. Hollinger, T. Laurila, A. Lindroth, A. D. Richardson, and T. Vesala (2008). "Net carbon dioxide losses of northern ecosystems in response to autumn warming”. Nature 451.7174, 49-52.

Piao, S., P. Friedlingstein, P. Ciais, P. Peylin, B. Zhu, and M. Reichstein (2009). "Footprint of temperature changes in the temperate and boreal forest carbon balance: Temperature and carbon balance". Geophysical Research Letters 36.7.

Poorter, L., F. Bongers, T. M. Aide, A. M. Almeyda Zambrano, P. Balvanera, J. M. Becknell, V. Boukili, P. H. S. Brancalion, E. N. Broadbent, R. L. Chazdon, D. Craven, J. S. de AlmeidaCortez, G. A. L. Cabral, B. H. J. de Jong, J. S. Denslow, D. H. Dent, S. J. DeWalt, J. M. Dupuy, S. M. Durán, M. M. Espírito-Santo, M. C. Fandino, R. G. César, J. S. Hall, J. L. Hernandez-Stefanoni, C. C. Jakovac, A. B. Junqueira, D. Kennard, S. G. Letcher, J.-C. Licona, M. Lohbeck, E. Marín-Spiotta, M. Martínez-Ramos, P. Massoca, J. A. Meave, R. Mesquita, F. Mora, R. Muñoz, R. Muscarella, Y. R. F. Nunes, S. Ochoa-Gaona, A. A. de Oliveira, E. Orihuela-Belmonte, M. Peña-Claros, E. A. Pérez-García, D. Piotto, J. S. Powers, J. RodríguezVelázquez, I. E. Romero-Pérez, J. Ruíz, J. G. Saldarriaga, A. Sanchez-Azofeifa, N. B. Schwartz, M. K. Steininger, N. G. Swenson, M. Toledo, M. Uriarte, M. van Breugel, H. van der Wal, M. D. M. Veloso, H. F. M. Vester, A. Vicentini, I. C. G. Vieira, T. V. Bentos, G. B. Williamson, and D. M. A. Rozendaal (2016). "Biomass resilience of Neotropical secondary forests". Nature 530.7589, 211-214.

Potter, C. S., J. T. Randerson, C. B. Field, P. A. Matson, P. M. Vitousek, H. A. Mooney, and S. A. Klooster (1993). "Terrestrial ecosystem production: A process model based on global satellite and surface data". Global Biogeochemical cycles 7.4, 811-841.

Poulter, B., L. Aragão, N. Andela, V. Bellassen, P. Ciais, T. Kato, X. Lin, B. Nachin, S. Luyssaert, N. Pederson, P. Peylin, S. Piao, T. Pugh, S. Saatchi, D. Schepaschenko, M. Schelhaas, and A. Shivdenko (2019). The global forest age dataset and its uncertainties (GFADv1.1). PANGAEA - Data Publisher for Earth \& Environmental Science. 
Poulter, B., D. Frank, P. Ciais, R. B. Myneni, N. Andela, J. Bi, G. Broquet, J. G. Canadell, F. Chevallier, Y. Y. Liu, S. W. Running, S. Sitch, and G. R. van der Werf (2014). "Contribution of semi-arid ecosystems to interannual variability of the global carbon cycle". Nature 509.7502, 600-603.

Pregitzer, K. S. and E. S. Euskirchen (2004). "Carbon cycling and storage in world forests: biome patterns related to forest age”. Global Change Biology 10.12, 2052-2077.

Premke, K., K. Attermeyer, J. Augustin, A. Cabezas, P. Casper, D. Deumlich, J. Gelbrecht, H. H. Gerke, A. Gessler, H.-P. Grossart, S. Hilt, M. Hupfer, T. Kalettka, Z. Kayler, G. Lischeid, M. Sommer, and D. Zak (2016). "The importance of landscape diversity for carbon fluxes at the landscape level: small-scale heterogeneity matters". Wiley Interdisciplinary Reviews: Water 3.4, 601-617.

Pugh, T. A. M., M. Lindeskog, B. Smith, B. Poulter, A. Arneth, V. Haverd, and L. Calle (2019). "Role of forest regrowth in global carbon sink dynamics". Proceedings of the National Academy of Sciences 116.10, 4382-4387.

Reichstein, M., S. Besnard, N. Carvalhais, F. Gans, M. Jung, B. Kraft, and M. Mahecha (2018). "Modelling Landsurface Time-Series with Recurrent Neural Nets". In: IGARSS 2018 - 2018 IEEE International Geoscience and Remote Sensing Symposium, 7640-7643.

Reichstein, M., P. Ciais, D. Papale, R. Valentini, S. Running, N. Viovy, W. Cramer, A. Granier, J. Ogée, V. Allard, M. Aubinet, C. Bernhofer, N. Buchmann, A. Carrara, T. Grünwald, M. Heimann, B. Heinesch, A. Knohl, W. Kutsch, D. Loustau, G. Manca, G. Matteucci, F. Miglietta, J. M. Ourcival, K. Pilegaard, J. Pumpanen, S. Rambal, S. Schaphoff, G. Seufert, J.-F. Soussana, M.-J. Sanz, T. Vesala, and M. Zhao (2007a). "Reduction of ecosystem productivity and respiration during the European summer 2003 climate anomaly: a joint flux tower, remote sensing and modelling analysis". Global Change Biology 13.3, 634-651.

Reichstein, M., M. Bahn, P. Ciais, D. Frank, M. D. Mahecha, S. I. Seneviratne, J. Zscheischler, C. Beer, N. Buchmann, D. C. Frank, D. Papale, A. Rammig, P. Smith, K. Thonicke, M. van der Velde, S. Vicca, A. Walz, and M. Wattenbach (2013). "Climate extremes and the carbon cycle". Nature 500.7462, 287-295.

Reichstein, M., G. Camps-Valls, B. Stevens, M. Jung, J. Denzler, N. Carvalhais, and Prabhat (2019). "Deep learning and process understanding for data-driven Earth system science". Nature 566.7743, 195.

Reichstein, M., E. Falge, D. Baldocchi, D. Papale, M. Aubinet, P. Berbigier, C. Bernhofer, N. Buchmann, T. Gilmanov, A. Granier, T. Grünwald, K. Havránková, H. Ilvesniemi, D. Janous, A. Knohl, T. Laurila, A. Lohila, D. Loustau, G. Matteucci, T. Meyers, F. Miglietta, J.-M. Ourcival, J. Pumpanen, S. Rambal, E. Rotenberg, M. Sanz, J. Tenhunen, G. Seufert, F. Vaccari, T. Vesala, D. Yakir, and R. Valentini (2005). "On the separation of net ecosystem exchange into assimilation and ecosystem respiration: review and improved algorithm". Global Change Biology 11.9, 1424-1439. 
Reichstein, M., D. Papale, R. Valentini, M. Aubinet, C. Bernhofer, A. Knohl, T. Laurila, A. Lindroth, E. Moors, K. Pilegaard, and G. Seufert (2007b). "Determinants of terrestrial ecosystem carbon balance inferred from European eddy covariance flux sites". Geophysical Research Letters 34.1.

Reichstein, M., A. D. Richardson, M. Migliavacca, and N. Carvalhais (2014). "Plant-Environment Interactions Across Multiple Scales". In: Ecology and the Environment. New York, NY: Springer New York, 1-27.

Richardson, A. D. and D. Y. Hollinger (2005). "Statistical modeling of ecosystem respiration using eddy covariance data: Maximum likelihood parameter estimation, and Monte Carlo simulation of model and parameter uncertainty, applied to three simple models". Agricultural and Forest Meteorology 131.3, 191-208.

Rödig, E., M. Cuntz, A. Rammig, R. Fischer, F. Taubert, and A. Huth (2018). "The importance of forest structure for carbon fluxes of the Amazon rainforest". Environmental Research Letters $13.5,054013$.

Rodríguez-Calcerrada, J., J.-M. Limousin, N. K. Martin-StPaul, C. Jaeger, and S. Rambal (2012). "Gas exchange and leaf aging in an evergreen oak: causes and consequences for leaf carbon balance and canopy respiration". Tree Physiology 32.4, 464-477.

Rumelhart, D. E., G. E. Hinton, and R. J. Williams (1986). "Learning representations by backpropagating errors". Nature 323.6088, 533.

Rußwurm, M. and M. Körner (2017). “Temporal Vegetation Modelling Using Long Short-Term Memory Networks for Crop Identification from Medium-Resolution Multi-spectral Satellite Images". In: 2017 IEEE Conference on Computer Vision and Pattern Recognition Workshops (CVPRW), 1496-1504.

Ryan, M. G., D. Binkley, and J. H. Fownes (1997). “Age-Related Decline in Forest Productivity: Pattern and Process". In: Advances in Ecological Research. Vol. 27. Academic Press, 213262.

Ryan, M. G., D. Binkley, J. H. Fownes, C. P. Giardina, and R. S. Senock (2004). “An Experimental Test of the Causes of Forest Growth Decline with Stand Age". Ecological Monographs 74.3, 393-414.

Ryo, M., C. A. Aguilar-Trigueros, L. Pinek, L. A. H. Muller, and M. C. Rillig (2019). "Basic Principles of Temporal Dynamics". Trends in Ecology \& Evolution.

Schepaschenko, D., L. See, M. Lesiv, I. McCallum, S. Fritz, C. Salk, E. Moltchanova, C. Perger, M. Shchepashchenko, A. Shvidenko, S. Kovalevskyi, D. Gilitukha, F. Albrecht, F. Kraxner, A. Bun, S. Maksyutov, A. Sokolov, M. Dürauer, M. Obersteiner, V. Karminov, and P. Ontikov (2015). "Development of a global hybrid forest mask through the synergy of remote sensing, crowdsourcing and FAO statistics". Remote Sensing of Environment 162, 208-220. 
Schepaschenko, D., A. Shvidenko, V. Usoltsev, P. Lakyda, Y. Luo, R. Vasylyshyn, I. Lakyda, Y. Myklush, L. See, I. McCallum, S. Fritz, F. Kraxner, and M. Obersteiner (2017). "A dataset of forest biomass structure for Eurasia". Scientific Data 4, 170070.

Schimel, D. (2007). "Carbon cycle conundrums". Proceedings of the National Academy of Sciences $104.47,18353-18354$.

Schimel, D., R. Pavlick, J. B. Fisher, G. P. Asner, S. Saatchi, P. Townsend, C. Miller, C. Frankenberg, K. Hibbard, and P. Cox (2015). "Observing terrestrial ecosystems and the carbon cycle from space”. Global Change Biology 21.5, 1762-1776.

Schmidhuber, J. (2015). “Deep Learning in Neural Networks: An Overview”. Neural Networks 61, 85-117.

Schmidt, G., C. Jenkerson, J. Masek, E. Vermote, and F. Gao (2013). Landsat ecosystem disturbance adaptive processing system (LEDAPS) algorithm description. Tech. rep. US Geological Survey.

Schwarz, P. A., B. E. Law, M. Williams, J. Irvine, M. Kurpius, and D. Moore (2004). "Climatic versus biotic constraints on carbon and water fluxes in seasonally drought-affected ponderosa pine ecosystems". Global Biogeochemical Cycles 18.4.

Seddon, A. W. R., M. Macias-Fauria, P. R. Long, D. Benz, and K. J. Willis (2016). "Sensitivity of global terrestrial ecosystems to climate variability". Nature 531, 229-232.

Seidl, R., W. Rammer, and T. A. Spies (2014). "Disturbance legacies increase the resilience of forest ecosystem structure, composition, and functioning". Ecological Applications 24.8, 2063-2077.

Seidl, R., M.-J. Schelhaas, and M. J. Lexer (2011). "Unraveling the drivers of intensifying forest disturbance regimes in Europe". Global Change Biology 17.9, 2842-2852.

Sheffield, J. and E. F. Wood (2008). "Projected changes in drought occurrence under future global warming from multi-model, multi-scenario, IPCC AR4 simulations". Climate Dynamics 31.1, 79-105.

Shen, W., G. D. Jenerette, D. Hui, and R. L. Scott (2016). "Precipitation legacy effects on dryland ecosystem carbon fluxes: direction, magnitude and biogeochemical carryovers". Biogeosciences 13.2, 425-439.

Shugart, H. H., B. Wang, R. Fischer, J. Ma, J. Fang, X. Yan, A. Huth, and A. H. Armstrong (2018). "Gap models and their individual-based relatives in the assessment of the consequences of global change". Environmental Research Letters 13.3, 033001.

Somogyi, Z., M. Teobaldelli, S. Federici, G. Matteucci, V. Pagliari, G. Grassi, and G. Seufert (2008). "Allometric biomass and carbon factors database". iForest-Biogeosciences and Forestry 1.3, 107.

Spies, T. A., J. F. Franklin, et al. (1991). "The structure of natural young, mature, and oldgrowth Douglas-fir forests in Oregon and Washington". Wildlife and vegetation of unmanaged Douglas-fir forests, 91-109. 
Srivastava, N., G. Hinton, A. Krizhevsky, I. Sutskever, and R. Salakhutdinov (2014). "Dropout: A Simple Way to Prevent Neural Networks from Overfitting". Journal of Machine Learning Research 15, 1929-1958.

Stephens, B. B., K. R. Gurney, P. P. Tans, C. Sweeney, W. Peters, L. Bruhwiler, P. Ciais, M. Ramonet, P. Bousquet, T. Nakazawa, S. Aoki, T. Machida, G. Inoue, N. Vinnichenko, J. Lloyd, A. Jordan, M. Heimann, O. Shibistova, R. L. Langenfelds, L. P. Steele, R. J. Francey, and A. S. Denning (2007). "Weak Northern and Strong Tropical Land Carbon Uptake from Vertical Profiles of Atmospheric CO2”. Science 316.5832, 1732-1735.

Stocker, B. D., J. Zscheischler, T. F. Keenan, I. C. Prentice, S. I. Seneviratne, and J. Peñuelas (2019). "Drought impacts on terrestrial primary production underestimated by satellite monitoring". Nature Geoscience, 1.

Sulla-Menashe, D., C. E. Woodcock, and M. A. Friedl (2018). "Canadian boreal forest greening and browning trends: an analysis of biogeographic patterns and the relative roles of disturbance versus climate drivers". Environmental Research Letters 13.1, 014007.

Sullivan, M. J. P., J. Talbot, S. L. Lewis, O. L. Phillips, L. Qie, S. K. Begne, J. Chave, A. CuniSanchez, W. Hubau, G. Lopez-Gonzalez, L. Miles, A. Monteagudo-Mendoza, B. Sonké, et al. (2017). "Diversity and carbon storage across the tropical forest biome". Scientific Reports 7 , 39102.

Sutskever, I., O. Vinyals, and Q. V. Le (2014). "Sequence to Sequence Learning with Neural Networks". arXiv:1409.3215.

Swinbank, W. C. (1951). "The measurement of vertical transfer of heat and water vapor by eddies in the lower atmosphere". Journal of Meteorology 8.3, 135-145.

Tang, J., S. Luyssaert, A. D. Richardson, W. Kutsch, and I. A. Janssens (2014). "Steeper declines in forest photosynthesis than respiration explain age-driven decreases in forest growth". Proceedings of the National Academy of Sciences 111.24, 8856-8860.

Team, R. C. (2015). R: A language and environment for statistical computing [Internet]. Vienna, Austria: R Foundation for Statistical Computing; 2014.

Thom, D., W. Rammer, R. Garstenauer, and R. Seidl (2018). "Legacies of past land use have a stronger effect on forest carbon exchange than future climate change in a temperate forest landscape". Biogeosciences 15.18, 5699-5713.

Thornton, P. E., B. E. Law, H. L. Gholz, K. L. Clark, E. Falge, D. S. Ellsworth, A. H. Goldstein, R. K. Monson, D. Hollinger, M. Falk, J. Chen, and J. P. Sparks (2002). "Modeling and measuring the effects of disturbance history and climate on carbon and water budgets in evergreen needleleaf forests". Agricultural and Forest Meteorology 113.1, 185-222.

Thuiller, W., S. Lavergne, C. Roquet, I. Boulangeat, B. Lafourcade, and M. B. Araujo (2011). "Consequences of climate change on the tree of life in Europe". Nature 470.7335, 531-534.

Tramontana, G., M. Jung, C. R. Schwalm, K. Ichii, G. Camps-Valls, B. Ráduly, M. Reichstein, M. A. Arain, A. Cescatti, G. Kiely, L. Merbold, P. Serrano-Ortiz, S. Sickert, S. Wolf, and 
D. Papale (2016). "Predicting carbon dioxide and energy fluxes across global FLUXNET sites with regression algorithms". Biogeosciences 13.14, 4291-4313.

Trumbore, S., P. Brando, and H. Hartmann (2015). "Forest health and global change". Science 349.6250, 814-818.

Tucker, C. J. (1979). "Red and photographic infrared linear combinations for monitoring vegetation". Remote Sensing of Environment 8.2, 127-150.

Turner, M. G. (2010). "Disturbance and landscape dynamics in a changing world”. Ecology 91.10, 2833-2849.

Vicca, S., M. Bahn, M. Estiarte, E. E. van Loon, R. Vargas, G. Alberti, P. Ambus, M. A. Arain, C. Beier, L. P. Bentley, W. Borken, N. Buchmann, S. L. Collins, G. de Dato, J. S. Dukes, C. Escolar, P. Fay, G. Guidolotti, P. J. Hanson, A. Kahmen, G. Kröel-Dulay, T. Ladreiter-Knauss, K. S. Larsen, E. Lellei-Kovacs, E. Lebrija-Trejos, F. T. Maestre, S. Marhan, M. Marshall, P. Meir, Y. Miao, J. Muhr, P. A. Niklaus, R. Ogaya, J. Peñuelas, C. Poll, L. E. Rustad, K. Savage, A. Schindlbacher, I. K. Schmidt, A. R. Smith, E. D. Sotta, V. Suseela, A. Tietema, N. van Gestel, O. van Straaten, S. Wan, U. Weber, and I. A. Janssens (2014). "Can current moisture responses predict soil $\mathrm{CO}_{2}$ efflux under altered precipitation regimes? A synthesis of manipulation experiments". Biogeosciences 11.11, 2991-3013.

Vicca, S., S. Luyssaert, J. Peñuelas, M. Campioli, F. S. Chapin, P. Ciais, A. Heinemeyer, P. Högberg, W. L. Kutsch, B. E. Law, Y. Malhi, D. Papale, S. L. Piao, M. Reichstein, E. D. Schulze, and I. A. Janssens (2012). "Fertile forests produce biomass more efficiently". Ecology Letters 15.6, 520-526.

Vicca, S., M. Balzarolo, I. Filella, A. Granier, M. Herbst, A. Knohl, B. Longdoz, M. Mund, Z. Nagy, K. Pintér, S. Rambal, J. Verbesselt, A. Verger, A. Zeileis, C. Zhang, and J. Peñuelas (2016). "Remotely-sensed detection of effects of extreme droughts on gross primary production". Scientific Reports 6.

Viovy, N. (2018). CRUNCEP Version 7 - Atmospheric Forcing Data for the Community Land Model. Research Data Archive at the National Center for Atmospheric Research, Computational and Information Systems Laboratory.

Vitousek, P. M. (1997). “Human Domination of Earth's Ecosystems”. Science 277.5325, 494499.

Wang, R., D. Goll, Y. Balkanski, D. Hauglustaine, O. Boucher, P. Ciais, I. Janssens, J. Penuelas, B. Guenet, J. Sardans, L. Bopp, N. Vuichard, F. Zhou, B. Li, S. Piao, S. Peng, Y. Huang, and S. Tao (2017). "Global forest carbon uptake due to nitrogen and phosphorus deposition from 1850 to 2100”. Global Change Biology 23.11, 4854-4872.

Ward, S. E., S. M. Smart, H. Quirk, J. R. B. Tallowin, S. R. Mortimer, R. S. Shiel, A. Wilby, and R. D. Bardgett (2016). "Legacy effects of grassland management on soil carbon to depth". Global Change Biology 22.8, 2929-2938. 
Werf, G. R. v. d., J. T. Randerson, L. Giglio, T. T. v. Leeuwen, Y. Chen, B. M. Rogers, M. Mu, M. J. E. v. Marle, D. C. Morton, G. J. Collatz, R. J. Yokelson, and P. S. Kasibhatla (2017). “Global fire emissions estimates during 1997-2016”. Earth System Science Data 9.2, 697-720.

Wieder, W. (2014). Regridded Harmonized World Soil Database v1.2. ORNL Distributed Active Archive Center.

Williams, C. A., G. J. Collatz, J. Masek, and S. N. Goward (2012). "Carbon consequences of forest disturbance and recovery across the conterminous United States". Global Biogeochemical Cycles 26.1.

Wirth, C., C. I. Czimczik, and E.-D. Schulze (2002). "Beyond annual budgets: carbon flux at different temporal scales in fire-prone Siberian Scots pine forests". Tellus B: Chemical and Physical Meteorology 54.5, 611-630.

Wolf, S., W. Eugster, C. Potvin, B. L. Turner, and N. Buchmann (2011). "Carbon sequestration potential of tropical pasture compared with afforestation in Panama". Global Change Biology 17.9, 2763-2780.

Woodall, C. W., M. B. Russell, B. F. Walters, A. W. D’Amato, S. Fraver, and G. M. Domke (2015). "Net carbon flux of dead wood in forests of the Eastern US". Oecologia 177.3, 861874.

Woodbury, P. B., J. E. Smith, and L. S. Heath (2007). "Carbon sequestration in the U.S. forest sector from 1990 to 2010”. Forest Ecology and Management. 241: 14-27. 241.

Woodwell, G. M. and R. H. Whittaker (1968). "Primary Production in Terrestrial Ecosystems". Integrative and Comparative Biology 8.1, 19-30.

Wu, J., L. P. Albert, A. P. Lopes, N. Restrepo-Coupe, M. Hayek, K. T. Wiedemann, K. Guan, S. C. Stark, B. Christoffersen, N. Prohaska, J. V. Tavares, S. Marostica, H. Kobayashi, M. L. Ferreira, K. S. Campos, R. d. Silva, P. M. Brando, D. G. Dye, T. E. Huxman, A. R. Huete, B. W. Nelson, and S. R. Saleska (2016). "Leaf development and demography explain photosynthetic seasonality in Amazon evergreen forests". Science 351.6276, 972-976.

Xiao, J., Q. Zhuang, E. Liang, X. Shao, A. D. McGuire, A. Moody, D. W. Kicklighter, and J. M. Melillo (2009). "Twentieth-Century Droughts and Their Impacts on Terrestrial Carbon Cycling in China”. Earth Interactions 13.10, 1-31.

Yi, C., D. Ricciuto, R. Li, J. Wolbeck, X. Xu, M. Nilsson, L. Aires, J. D. Albertson, C. Ammann, M. A. Arain, A. C. d. Araujo, M. Aubinet, M. Aurela, et al. (2010). "Climate control of terrestrial carbon exchange across biomes and continents". Environmental Research Letters 5.3, 034007.

Yu, G., Z. Chen, S. Piao, C. Peng, P. Ciais, Q. Wang, X. Li, and X. Zhu (2014). "High carbon dioxide uptake by subtropical forest ecosystems in the East Asian monsoon region". Proceedings of the National Academy of Sciences 111.13, 4910-4915. 
Zeng, Z., L. Estes, A. D. Ziegler, A. Chen, T. Searchinger, F. Hua, K. Guan, A. Jintrawet, and E. F. Wood (2018). "Highland cropland expansion and forest loss in Southeast Asia in the twenty-first century". Nature Geoscience 11.8, 556-562.

Zhang, B., X. Zhou, L. Zhou, and R. Ju (2015a). "A global synthesis of below-ground carbon responses to biotic disturbance: a meta-analysis: Below-ground $\mathrm{C}$ responses to biotic disturbance". Global Ecology and Biogeography 24.2, 126-138.

Zhang, T., M. Xu, Y. Xi, J. Zhu, L. Tian, X. Zhang, Y. Wang, Y. Li, P. Shi, G. Yu, X. Sun, and Y. Zhang (2015b). "Lagged climatic effects on carbon fluxes over three grassland ecosystems in China". Journal of Plant Ecology 8.3, 291-302.

Zhang, Y., Y. Yao, X. Wang, Y. Liu, and S. Piao (2017). "Mapping spatial distribution of forest age in China". Earth and Space Science 4.3, 108-116.

Zhao, M. and S. W. Running (2010). "Drought-Induced Reduction in Global Terrestrial Net Primary Production from 2000 Through 2009”. Science 329.5994, 940-943.

Zhou, D., S. Q. Zhao, S. Liu, and J. Oeding (2013). “A meta-analysis on the impacts of partial cutting on forest structure and carbon storage”. Biogeosciences 10.6, 3691-3703.

Zhu, Z., S. Piao, R. B. Myneni, M. Huang, Z. Zeng, J. G. Canadell, P. Ciais, S. Sitch, P. Friedlingstein, A. Arneth, C. Cao, L. Cheng, E. Kato, C. Koven, Y. Li, X. Lian, Y. Liu, R. Liu, J. Mao, Y. Pan, S. Peng, J. Peñuelas, B. Poulter, T. A. M. Pugh, B. D. Stocker, N. Viovy, X. Wang, Y. Wang, Z. Xiao, H. Yang, S. Zaehle, and N. Zeng (2016). "Greening of the Earth and its drivers". Nature Climate Change 6.8, 791-795.

Zhu, Z., S. Wang, and C. E. Woodcock (2015). "Improvement and expansion of the Fmask algorithm: cloud, cloud shadow, and snow detection for Landsats 4-7, 8, and Sentinel 2 images". Remote Sensing of Environment 159, 269-277.

Zhu, Z. and C. E. Woodcock (2012). "Object-based cloud and cloud shadow detection in Landsat imagery”. Remote Sensing of Environment 118, 83-94.

Zielis, S., S. Etzold, R. Zweifel, W. Eugster, M. Haeni, and N. Buchmann (2014). "NEP of a Swiss subalpine forest is significantly driven not only by current but also by previous year's weather". Biogeosciences 11.6, 1627-1635.

Zscheischler, J., M. D. Mahecha, V. Avitabile, L. Calle, N. Carvalhais, P. Ciais, F. Gans, N. Gruber, J. Hartmann, M. Herold, K. Ichii, M. Jung, P. Landschützer, G. G. Laruelle, R. Lauerwald, D. Papale, P. Peylin, B. Poulter, D. Ray, P. Regnier, C. Rödenbeck, R. M. RomanCuesta, C. Schwalm, G. Tramontana, A. Tyukavina, R. Valentini, G. v. d. Werf, T. O. West, J. E. Wolf, and M. Reichstein (2017). "Reviews and syntheses: An empirical spatiotemporal description of the global surface-atmosphere carbon fluxes: opportunities and data limitations". Biogeosciences 14.15, 3685-3703. 



\section{Summary}

Understanding the dynamics of terrestrial ecosystems in a changing environment is critical because of their fundamental role in the global carbon (C) cycle. Climate extremes, ecological disturbances, and anthropogenic activities are currently altering the functioning of terrestrial ecosystems. As a result, there is a need to improve the monitoring of the terrestrial ecosystem's and the role of extreme events (i.e. natural and human-induced disturbances) in the biogeochemical cycles for better quantifying regional and global $\mathrm{C}$ dynamics. In recent years, there has been an intensive global effort to measure and model carbon dioxide $\left(\mathrm{CO}_{2}\right)$ exchanges between the terrestrial biosphere and the atmosphere. The integration of multiple modeling methods, remote sensing data, climate data, and a global network of eddy-covariance (EC) flux towers has provided unprecedented insights in understanding the mechanisms controlling $\mathrm{CO}_{2}$ fluxes from ecosystem to regional scales. However, current bottom-up approaches do not explicitly account for the effects of site history (e.g. forest age) and the ecological memory effects of both vegetation and climate dynamics on $\mathrm{CO}_{2}$ fluxes.

Although most scientists agree on the importance of forest age and ecological memory effects in controlling the $\mathrm{CO}_{2}$ flux variability, there is still a debate about the quantitative role of forest age and ecological memory effects in estimating $\mathrm{CO}_{2}$ fluxes. In my thesis, I explored ways both to integrate forest age as well as ecological memory effects and to quantify their relevance when estimating the spatiotemporal variability of the $\mathrm{CO}_{2}$ fluxes. This was approached from two directions. First, a statistical method based on a combination of climate, ancillary, and EC data was developed to quantify the role of forest age towards the terrestrial net $\mathrm{CO}_{2}$ fluxes. Second, the application of a deep learning (DL) method was explored for understanding the contribution of vegetation and climate's ecological memory effects on $\mathrm{CO}_{2}$ fluxes.

In Chapter 2, I carried-out an observational synthesis to determine to what extent environmental conditions and site history (i.e. forest age) influence the spatiotemporal variability of forest annual net ecosystem production (NEP) across a set of forest EC flux sites globally. The proposed empirical model yielded a substantial capacity for reproducing the spatiotemporal (Nash-Sutcliffe model efficiency (NSE) of 0.62) and across-site variability (NSE of 0.71) of annual forest NEP. By investigating the model structure, I found that forest age was the main driver of NEP spatiotemporal variability in both space and time (decrease in NSE of 0.42 and 0.50 for spatiotemporal and across-site variability, respectively). These results confirmed 
the importance of forest age in quantifying spatiotemporal variation in NEP using data-driven approaches and paved the way towards further developments in upscaling EC data. Based on the findings of Chapter 2, I provided new global estimates of forest $\mathrm{C}$ balance by accounting for both forest age and climate spatial variations (chapter 3). Gridded estimates of forest NEP inferred from a new forest age map and environmental gridded global products (i.e. air temperature, gross primary production, and nitrogen deposition) at $0.5^{\circ}$ spatial resolution for the period $2000-2013$ were produced. This approach estimated the global forest NEP as a sink of around $+5 \pm 0.2 \mathrm{PgC}$ $\mathrm{yr}^{-1}$ and the net biome production (NBP) of forests of around $+3 \pm 0.3 \mathrm{PgC} \mathrm{yr}^{-1}$. Forest NBP estimates matched results of independent forest inventories globally, while discrepancies were found at biome level (i.e. temperate, boreal, and tropical regions). Overall, this first attempt to include forest age for estimating the forest $\mathrm{C}$ balance globally provided new insights on both the location and the magnitude of the global land $\mathrm{C}$ sink.

Furthermore, I investigated the relevance of capturing the vegetation and climate temporal properties, the so-called ecological memory effects, for predicting net ecosystem exchange (NEE) at 185 forest and woodland FLUXNET sites (Chapter 4). To answer this question, I used a data-driven DL model that translates the response of net $\mathrm{CO}_{2}$ fluxes to past climate and vegetation fluctuations: Long-Short-Term Memory (LSTM) model. The findings of the experiments were two folds: (1) an LSTM approach with embedded climate and vegetation ecological memory effects outperforms a non-dynamic statistical model (i.e. Random Forest) and (2) the vegetation mean seasonal cycle embeds most of the information content to realistically explain the spatial and seasonal variations in NEE. To further explore the contribution of vegetation and climate's temporal dynamic properties to $\mathrm{CO}_{2}$ fluxes globally (Chapter 5), I expanded the approach developed in Chapter 4. A bottom-up approach and a series of experiments provided evidence with respect to the geographical distribution and magnitude of vegetation and climate's ecological memory effects on NEE for the 2001-2018 period. The spatial patterns of ecological memory effects as well as the controls of the ecosystem properties and climatic conditions on the observed ecological memory effects' spatial patterns were explored. The results depicted widespread and substantial ecological memory effects across the globe, confirming the importance of explicitly capturing the vegetation and climate temporal properties to accurately reproduce $\mathrm{CO}_{2}$ flux spatiotemporal patterns. Finally, I explored to what degree vegetation and climate's ecological effects control the biosphere-atmosphere $\mathrm{CO}_{2}$ responses to a specific climate extreme event (i.e. 2018 European heatwave).

Chapter 6 summarized the main findings of the thesis and provided additional reflections as well as outlooks for future research. Overall, this thesis strengthened the role of ecosystem history in understanding the biosphere-atmosphere $\mathrm{CO}_{2}$ exchange. Methodologically, my works have demonstrated the potential of new modeling approaches in the Earth system science, such as DL. Yet, the accommodation of new data streams in the presented modeling schemes and the development of new model frameworks are of relevance. Thereby, the potential of integrating new datasets (e.g. biomass time-series, soil moisture) for investigating the control of $\mathrm{C}$ stocks and soil moisture stress on $\mathrm{CO}_{2}$ fluxes for providing more reliable global $\mathrm{CO}_{2}$ fluxes products were 
briefly presented in Chapter 6. In addition, the potential application of a data-driven method (i.e. transfer learning) for overcoming the problem of extrapolation when modeling $\mathrm{CO}_{2}$ fluxes from site to globe was introduced. This chapter also pointed at the design of modeling schemes that are not only data-adaptive but also embed physical ecosystem properties, the so-called hybrid models. Finally, a brief reflection on the feasibility of implementing operational systems for $\mathrm{CO}_{2}$ flux monitoring was provided. 



\section{Acknowledgements}

Some energy left after reading the 180 pages of my thesis and reflecting on the concepts of ecosystem history and biosphere-atmosphere carbon dioxide $\left(\mathrm{CO}_{2}\right)$ exchange? Here comes the part where all the persons that have contributed in many ways to my dissertation are acknowledged. I will possibly forget to mention individuals that made the past four years of my personal and professional life enjoyable. If so, I will happily buy you a drink and beg you for forgiveness.

First and foremost I want to thank Martin Herold for giving me the opportunity to pursue my dream of becoming a scientist. He did not only organise the fundings to finance my Ph.D. research but also gave me a chance to work in collaboration with the Laboratory of Remote sensing, Wageningen University, and the Department Biogeochemical Integration, Max Planck Institute for Biogeochemistry. This collaboration allowed me to carry out my research in the best scientific conditions possible. I also would like to thank Nuno Carvalhais for his support during my journey. He has taught me, both consciously and unconsciously, how to become a good scientist and how to constantly reflect on the meaning of what I was doing scientifically. I appreciate all his contributions of time and ideas to make my Ph.D. experience productive and stimulating. The joy and enthusiasm he has for his research were contagious and motivational for me, even during tough times in the Ph.D. pursuit. This will probably sound cliché but I would have never been able to achieve what I have done within four years without him. Obrigado Nuno! I am also thankful to Markus Reichstein for his valuable advice during my Ph.D. and for introducing me to Deep Learning. In the end, a short meeting in his office talking about LSTMs led to two key chapters of my Ph.D. thesis. I also deeply value his leadership and continuous curiosity, which undoubtedly contribute to the success of the BGI department. I am also grateful to Jan Clevers who was always ready to help me even though the content of my Ph.D. thesis did not always relate to his scientific background.

The members of the Max Planck Institute for Biogeochemistry have contributed immensely to my personal and professional time. I am especially grateful for the members of the MDI and GDM groups. These groups have been a source of friendships as well as good advice and collaboration. I will especially remember the Tuesday MDI meetings during which a lot of ideas (mostly good) were discussed and ended up in the presented thesis. I have also appreciated the camaraderie of the Laboratory of Remote and Geo-information Science at the University 
of Wageningen. Special thanks to my office mates both at the Laboratory of Remote sensing and the Department Biogeochemical Integration: Mathieu, Andrei, John, Richard, Robert, Tina, Jasper, and Junzhi. I also would like to thank Truus, Antoinette, Birgitta, Linda, and Alice for their time and patience dealing with my administrative, financial, and traveling questions.

While being far away from my family during the last four years, I have met persons that made me feel like home either in the Netherlands or Germany. Not that being a Ph.D. student is an insurmountable experience but the times I spent with the Wageningen and Utrecht's crews were key in breaking the occasional monotony of my Ph.D. life and overcoming some of the tough days I faced. All the barbecues, parties, concerts, or drinks spent with any of you brought me love, joy, and fun that I really needed to move forward and overcome the hurdles encountered during every step of my Ph.D. Maíra, Thaïs, Duda, Montse, Dudu, Alice, Loïc, Nelson, Marcell, Björn, Memê, Fran, Alfonso, Alba, Antonella, Sara, Bram, Patri, Lucas, Mariana, Faheem, Que Beleza members and fans as well as the Herenstraat 14 and Pad van Witte communities, I am glad to have you around me and I hope to share more good moments with all of you. Jake, Tiana, Berni, Sujan, and Milie, my visits in Jena would have not been the same without you. The times we had together made Jena a special place for me. So many incredible stories in my head (and some are probably real) that I will not even dare to tell my kids. Special thanks to Jake who became not only a colleague but a very good friend. Climbing the Ph.D. mountain together from the beginning was an enriching experience professionally and personally. I am glad none of us fell from the cliff. Karola and Gerd, I have really appreciated your support and kindness towards me during the last four years.

Living abroad for the last four years also meant being physically distant from my childhood friends of my dear Brittany. Gosh, I have missed you guys so much during the last four years and being far away from you was harder than I expected. There is no doubt that the friendship we have is unique. The too few moments we can spend together are always memorable and always remind me that you all occupy a special place in my heart. Pierrot and Jerem', who left us too early, I feel that I got where I am now somehow because of you. We miss you here!

Special notes to my two paranymphs: Maíra and Duda. Maíra, you called me in your thesis: "the French with a Brazilian soul". Well, I couldn't agree more but my love for Brazil and the Brazilians is definitely fed by your happiness, calmness, fight for gender equity, passion for saideiras, and love for music. The time I had in Pad van Witte, where we got the chance to develop our friendship, was unforgetable. Remember the second time we tried to make beers at Duda's place or our ways back home from Dezaaire? These ones were epic. Duda, we first met in my little student kitchen and since then we have shared many great moments together. You and Montse always have your door opened for coffee, cakes, dinners, and last minute sleep overs. You are a beautiful and inspiring family for many of us. Duda! We really have to find another hobby together than drinking wines. Maybe teaching you how to make a proper barbecue table or brewing beers while being sober? Yet, I do not get enough of our dinners where liters of wine are (probably excessively) consumed. That being said I am afraid I will continue opening the 
best wines you have in your cellar. Maíra and Duda, it is good to have you as friends and I am really looking forward to keeping spending good quality time together in Europe, Brazil, Mexico or elsewhere.

Ô Maren! Mon amour, it is hard to express all I want in a couple of sentences. Here is an attempt to write down the essentials. It is usually said that obtaining a Ph.D. is a scientific team effort but we tend to forget the important role of the partners during this journey. Sharing my life with you during my Ph.D. contributed substantially to what I have achieved. Although we were probably not aware of all the difficulties that a Ph.D. can carry over, you never stopped believing in me during the ups and downs. If one person had no doubt that I will be able to successfully finish my thesis, it was you! Your love, happiness, and encouragement meant a lot to me. The fact that you always bring joy, kindness, and calmness to the people around you made definitely the process of pursuing my Ph.D. easier. All the nice breakfasts you prepared on Saturday mornings associated with your lovely smile are perfect examples.

Lastly, I would like to thank my family for all their love and encouragement. Pour mes parents, Christian et Marie-Christine, qui m'ont soutenu dans toutes mes activités. Je me suis fréquemment demandé si vous avez toujours été en accord avec toutes mes décisions professionnelles et personnelles, mais vous ne m'avez jamais fait sentir que je prenais les mauvaises. Merci de ne jamais avoir remis en question mes choix et d'avoir été toujours là quand j'en avais besoin. Je vous suis également très reconnaissant pour toutes les valeurs que vous m'avez enseignées. Il y a un célèbre dicton espagnol qui dit ceci: mi casa es su casa. Ce proverbe représente assez bien l'éducation que vous m'avez donnée. Le partage et la générosité sont des valeurs importantes dans notre famille et je suis fier de porter cet héritage avec moi. Merci papa et maman! Pour mon frère, Sylvain, et Anne-Sophie avec qui j'aimerais pouvoir passer plus de temps, ainsi qu'avec mon neveu Jules et mes deux nièces Leonie et Lison. Enfin, j' aimerais avoir une pensée pour mes grands-parents, mes tantes, mes oncles et mes cousins. Je vous promets qu'un jour, j'essaierai de vous expliquer en quoi consistent mes contributions scientifiques.

It has been a priviliege for me to have shared this journey with all of you.

Thank you! 



\section{About the author}

Simon Besnard was born in Loudeac, France on March 14, 1986. His career began when he was around 8 years old, as a kind of apprentice at his uncle's farm. He remembers the weekends he spent during his childhood, learning how plants grow and how they interact with the soil. He eventually attended an agricultural university and then obtained two Master's degrees in Tropical Agrarian Systems as well as in Remote Sensing and Geo-information Science. Besides his education, he acquired professional experience in the field of agricul-

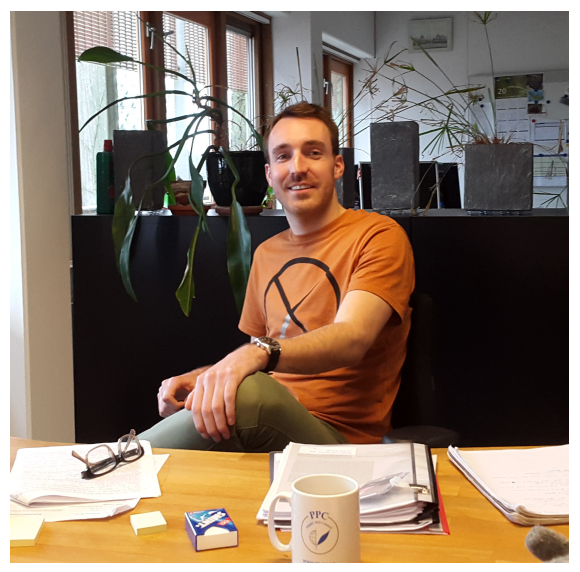
ture development in different research institutes and sustainable development projects.

During his education, he was trained with both theoretical and practical knowledge. The combination of theory and practice provided him with knowledge in natural resource management, farming, ecology, and hydrology. His rural upbringing and the studies he has undertaken on systemic approaches to agriculture supported and confirmed his interest in agriculture development and natural resource management. First, he interned at the National Institute for Agricultural Research (INRA) to study water conductivity in silty soils in till and no-till practices to understand the impact of these farming practices in soil water conductivity. Then, he worked with the Spanish NGO CERAI (Rural Centre for the Study of International Agriculture) in Tunisia, where he led the analysis of two different agrarian (irrigated) systems. This unforgettable experience motivated him to bolster his knowledge and skills in agriculture development and the management of natural resources. He then decided to pursue a Master's degree in Tropical Agrarian Systems with a major in International Land Management from the Institut des régions chaudes (Montpellier SupAgro), which is well known for its systemic approach to studying the relations between agriculture and natural resources. As a result, he learned how to understand farming systems and their impact on natural resources from an interdisciplinary approach.

With this technical and agricultural background, he decided to do his first Master's thesis in South Africa investigating land degradation processes in a former homeland in the province of 
KwaZulu-Natal. It was his understanding that in South Africa, today's access and distribution of land and water resources are a direct result of historical processes marked by the apartheid era. Thus, he believed South Africa was, perhaps, one of the most interesting cases of land management and degradation because of the ongoing land reforms, equity issues, and the distribution of land resources.

Thereafter, Simon started to develop interests in working on climate change mitigation and soil conservation, both as a consultant for Global Green Carbon Corporation in Nicaragua and with Environmental Defense Fund (EDF) in California. As a project manager, he developed a carbonfinanced AFOLU (Agriculture, Forestry, and Other Land Uses) project in the most difficult and remote area in the tropical rainforest ecosystem of Nicaragua. The objective of this project was to implement land management strategies addressing one of the primary causes of global warming, deforestation, but also to rehabilitate degraded ecosystems and provide opportunities for forest communities. He continued his interest in environmental conservation practices and climate change mitigation by working with EDF and its Working Land program. He did background research that helped EDF better work with ranchers to help them earn incentives for being environmental stewards for their land. His research mostly focused on soil carbon sequestration projects, particularly on how carbon standards deal with permanence and additionality for these types of initiatives.

After analyzing the drivers of deforestation and their role in global gross carbon (C) emissions during his second Master's thesis at Wageningen University, Simon started his Ph.D. in terrestrial ecosystems and the C cycle in March 2015. Over the course of his Ph.D., Simon has built up expertise in carbon dioxide $\left(\mathrm{CO}_{2}\right)$ flux measurements, climate, and remote sensing data as well as in developing different statistical modeling frameworks for understanding the role of ecosystem history on biosphere-atmosphere $\mathrm{CO}_{2}$ exchange. His work resulted in four scientific articles, two of which have been already published, while the other two are under review. Additionally, Simon presented his work at international conferences such as American Geophysical Union fall meeting, European Geophysical Union, and Living Planet Symposium. Simon's current research interests are related to the link between changes in $\mathrm{C}$ stocks and $\mathrm{CO}_{2}$ flux dynamics. This includes analyzing long-term time series of aboveground biomass (AGB) and incorporating such data streams into statistical and process-based models for understanding the contribution of $\mathrm{C}$ stock changes to the global $\mathrm{C}$ cycle.

\section{Peer-reviewed Journal Publications}

Besnard, S., N. Carvalhais, M. A. Arain, A. Black, B. Brede, N. Buchmann, J. Chen, J. G. P. W. Clevers, L. P. Dutrieux, F. Gans, M. Herold, M. Jung, Y. Kosugi, A. Knohl, B. E. Law, E. Paul-Limoges, A. Lohila, L. Merbold, O. Roupsard, R. Valentini, S. Wolf, X. Zhang, and M. Reichstein (2019a). "Memory effects of climate and vegetation affecting net ecosystem CO2 fluxes in global forests". PLOS ONE 14.2, e0211510. 
Besnard, S., N. Carvalhais, M. A. Arain, A. Black, S. d. Bruin, N. Buchmann, A. Cescatti, J. Chen, J. G. P. W. Clevers, A. R. Desai, C. M. Gough, K. Havrankova, M. Herold, L. Hörtnagl, M. Jung, A. Knohl, B. Kruijt, L. Krupkova, B. E. Law, A. Lindroth, A. Noormets, O. Roupsard, R. Steinbrecher, A. Varlagin, C. Vincke, and M. Reichstein (2018). "Quantifying the effect of forest age in annual net forest carbon balance". Environmental Research Letters 13.12, 124018.

\section{Other Scientific Publications}

Besnard, S., N. Carvalhais, J. G. Clevers, L. P. Dutrieux, F. Gans, M. Herold, M. Jung, and M. Reichstein (2017). "Modelling effects of forest disturbance history on carbon balance: a deep learning approach using Landsat-time series." In: AGU Fall Meeting Abstracts, New Orleans, USA, 11-15 December 2017.

Besnard, S., N. Carvalhais, J. G. Clevers, F. Gans, M. Herold, M. Jung, U. Weber, and M. Reichstein (2019b). "Memory effects of climate and vegetation affecting net ecosystem CO2 fluxes in global forests". In: Living Planet Symposium, Milan, Italy, 13-17 May 2019.

Besnard, S., N. Carvalhais, J. G. Clevers, M. Herold, M. Jung, and M. Reichstein (2016). "Stand age and climate drive forest carbon balance recovery". In: EGU General Assembly Conference Abstracts, Vienna, Austria, 17-22 April 2016.

Ciais, P., Y. Yao, S. Besnard, C. Yue, N. Carvalhais, B. Poulter, C. Jerome, P. Stoy, M. Jung, M. Herold, R. L. Chazdon, and M. Reichstein (2018). "The global carbon balance of forests based on flux towers and forest age data". In: Integrated Carbon Observation System, Prague, Czech Republic, 11-13 September 2018.

Mora, B., S. Besnard, and M. Herold (2014). Comparison of global urban maps in the context of the Land Cover Climate Change Initiative-Phase 2. Tech. rep. GOFC-GOLD Land Cover Project Office, Wageningen University, The Netherlands.

Reichstein, M., S. Besnard, N. Carvalhais, F. Gans, M. Jung, B. Kraft, and M. Mahecha (2018). "Modelling Landsurface Time-Series with Recurrent Neural Nets". In: 2018 IEEE International Geoscience and Remote Sensing Symposium, Valencia, Spain, 22-27 July 2018, 7640 7643.

Sy, V. de, M. Herold, R. Beuchle, S. Besnard, J. Clevers, E. Lindquist, L. Verchot, and A. Wijaya (2015). "Drivers of deforestation in South America: first results from a pan-tropical remote sensing analysis". In: 36th International Symposium on Remote Sensing of Environment, Berlin, Germany, 11-15 May 2015.

Walther, S., M. Jung, P. Bodesheim, J. Nelson, N. Carvalhais, M. Migliavacca, S. Besnard, U. Weber, and M. Reichstein (2019). "Advances in data-driven modeling of terrestrial carbon fluxes: resolving the diurnal cycle and efforts towards FLUXCOM 2.0". In: Living Planet Symposium, Milan, Italy, 13-17 May 2019. 



\section{PE\&RC Training and Education Statement}

With the training and education activities listed below the PhD candidate has complied with the requirements set by the C.T. de Wit Graduate School for Production Ecology and Resource Conservation (PE\&RC) which comprises of a minimum total of 32 ECTS (= 22 weeks of activities)

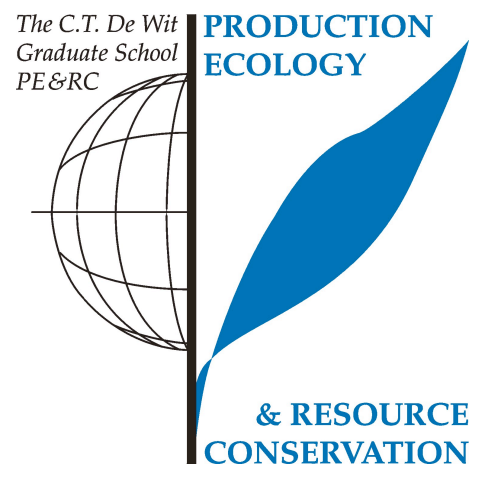

\section{Review of literature (6 ECTS)}

- The global carbon cycle, forests and disturbances: monitoring of terrestrial ecosystems

\section{Writing of project proposal (4.5 ECTS)}

- Tracking disturbance: understanding the role of forest disturbance and recovery on forest carbon cycle dynamics inferred by flux-data, remote sensing and modelling.

\section{Post-graduate courses (4.2 ECTS)}

- Eddy Covariance Workshop; International Max Planck Research School for Global Biogeochemical Cycles (2015)

- Applied statistics and data analysis; International Max Planck Research School for Global Biogeochemical Cycles (2015)

- Deep learning course; Michael Stifel Center Jena (2016)

- Bayesian Statistics; PE\&RC (2016)

- Programming in Matlab; BioSB (2017)

Laboratory training and working visits (4.5 ECTS)

- Visit to Max Planck Institute for biogeochemistry (2015-2019) 
Invited review of (unpublished) journal manuscript (1 ECTS)

- Canadian Journal of Forest Research: Fire disturbance history improves the consistency of remotely sensed aboveground biomass estimates for boreal forests in eastern Canada (2016)

\section{Competence strengthening / skills courses (2.1 ECTS)}

- Scientific writing course; Wageningen in'to Languages (2016)

- Debate training and practice tournament; Wageningen Graduate schools (2018)

PE\&RC Annual meetings, seminars and the PE\&RC weekend

\section{(1.65 ECTS)}

- PE\&RC workshop: Monitoring and Assessing Land Use Changes (2016)

- PE\&RC workshop: Space-time approaches in earth observations big data analysis (2017)

- Annual PE\&RC Day: Preventing the end of the world (2017)

- PE\&RC workshop: Humans shape tropical forests (2018)

- PE\&RC PhD Weekend (2018)

- PE\&RC workshop: Remote Sensing and Forest Resource Monitoring (2018)

- PE\&RC workshop: Computer vision for Geo-Information (2019)

Discussion groups / local seminars / other scientific meetings (5.5 ECTS)

- BGC seminar at Max Planck Institute for biogeochemistry (2015-2019)

- Thematic remote sensing group, WUR (2015-2019)

\section{International symposia, workshops and conferences (13.5 ECTS)}

- European Geosciences Union General Assembly; poster presentation; Vienna, Austria (2016)

- FLUXNET Workshop; poster presentation; Berkeley, United States (2017)

- FLUXCOM workshop; oral presentation; Jena, Germany (2017)

- American Geosciences Union General Assembly; poster presentation; New Orleans, United States (2017)

- European Geosciences Union General Assembly; poster presentation; Vienna, Austria (2019)

- Living Planet Symposium; oral presentation; Milan, Italy (2019)

\section{Supervision of MSc students (6 ECTS)}

- Elke Hendrix: Fire risk estimation of tropical rainforest using resilience metrics (2018) 

This research received funding from the BACI project and the Independent Monitoring Project.

Cover design by Nicole Schyns, The Netherlands | www.behance.net/nicolepietruschka 